\title{
Mechanisms underlying the temporal and selective induction of Ptf1a target genes
}

\author{
Doctoral Thesis \\ Dissertation for the award of the degree \\ "Doctor rerum naturalium (Dr. rer. nat.)" \\ in the GGNB program "Genes and Development" \\ at the Georg August University Göttingen \\ Faculty of Biology \\ submitted by \\ Sven Richts \\ born in Werl, Germany
}

Göttingen, December 2017 



\section{Members of the Thesis Committee:}

Dr. Kristine A. Henningfeld (Supervisor, Reviewer)

Department of Developmental Biochemistry,

University of Göttingen

Prof. Dr. Steven Johnsen (Reviewer)

Clinic for General, Visceral and Pediatric Surgery, University of Göttingen

Prof. Dr. Thomas Dresbach

Department for Anatomy and Embryology

University of Göttingen 


\section{Affidavit}

Herewith, I declare that I prepared the PhD thesis "Mechanisms underlying the temporal and selective induction of Ptf1a target genes " on my own and with no other sources and aids than quoted.

Submission date

Göttingen, 31.12.2017

Sven Richts 
Table of contents

Table of contents ................................................................................. I

Acknowledgements .......................................................................... VI

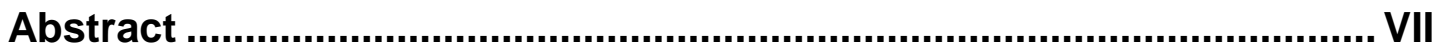

List of figures ..................................................................................... VIII

List 0 t tables...................................................................................... X

Abbreviations ..................................................................................... XII

1. Introduction .................................................................................... 1

1.1 Neurogenesis in Xenopus laevis............................................... 1

1.2 Neural induction ............................................................................ 2

1.3 bHLH transcription factors and their role during neuronal differentiation and determination ......................................................... 4

1.4 Lateral inhibition ......................................................................... 8

1.5 Neuronal subtype specification.................................................. 10

1.6 Role of phosphorylation in regulating neurogenesis................... 13

1.7 Epigenetic regulation of neurogenesis...................................... 14

1.8 Changes in the epigenetic landscape during development.......... 19

1.9 Ptf1a........................................................................................... 20

1.10 Ptf1a forms a trimeric transcription factor complex.................. 23

1.11 Gene induction and regulation by Ptf1a .................................. 24

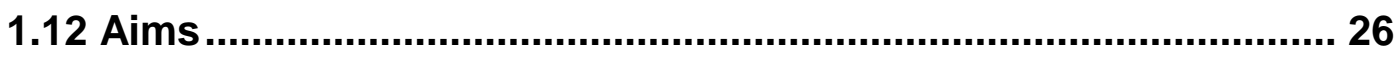

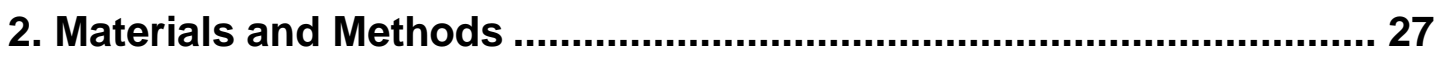

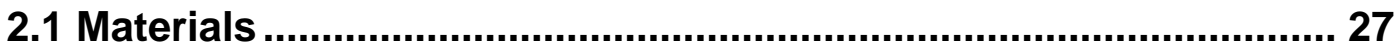

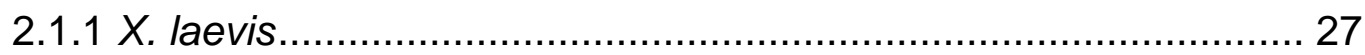

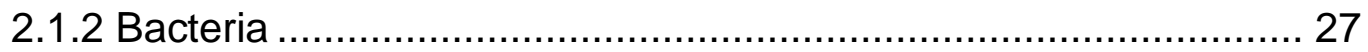

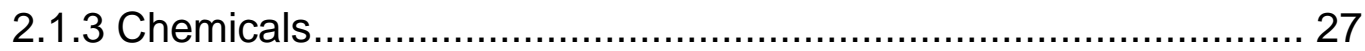

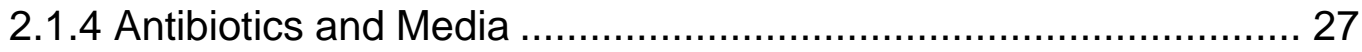




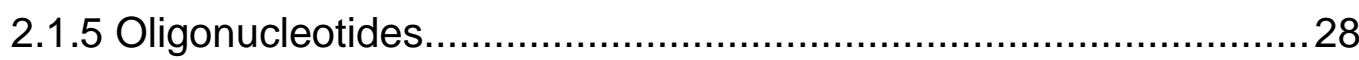

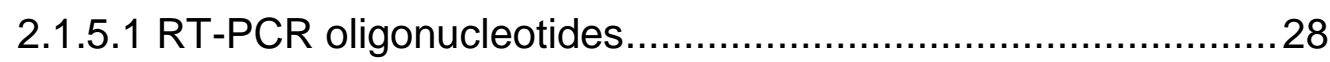

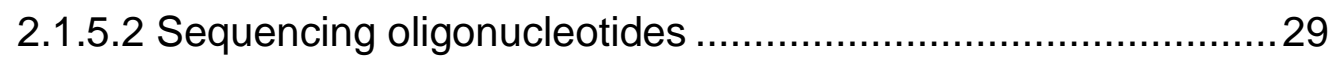

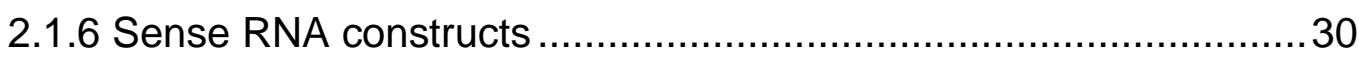

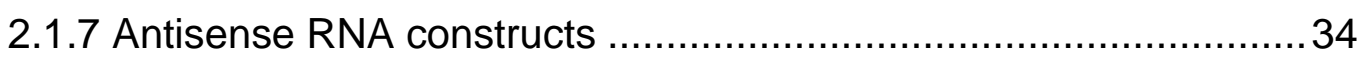

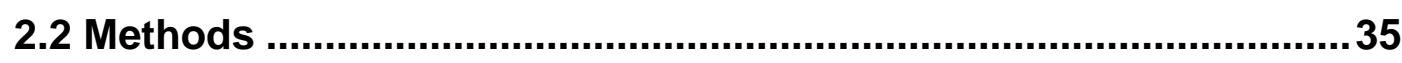

2.2.1 In vitro synthesis of capped sense RNA.....................................35

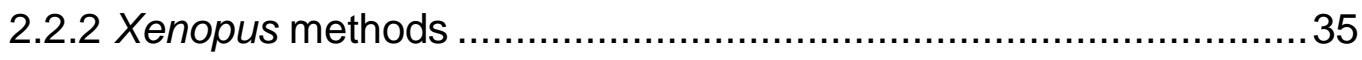

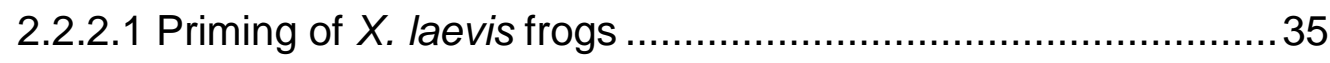

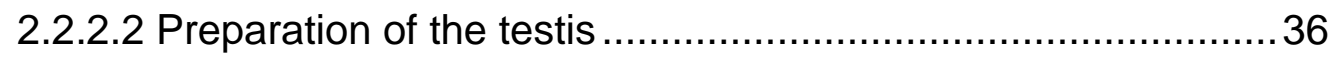

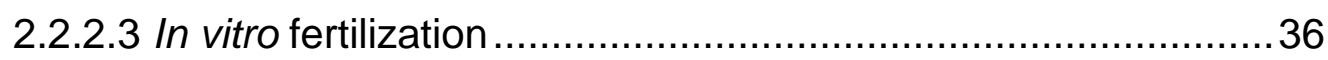

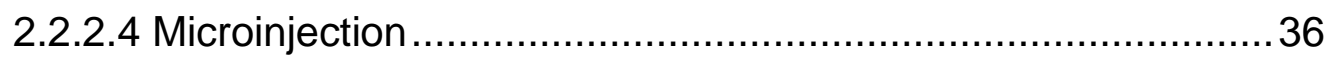

2.2.2.5 Preparation of ectodermal explants (animal caps) ..................36

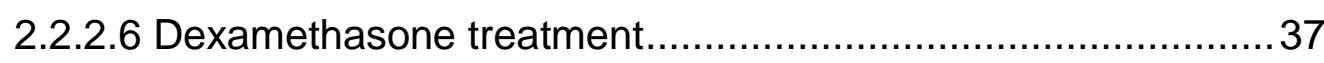

2.2.2.7 Bimolecular Fluorescent Complementation assay (BiFC) .......37

2.2.2.8 In vitro synthesis of antisense RNA ........................................

2.2.2.9 Whole mount in-situ hybridization (WMISH) ..........................38

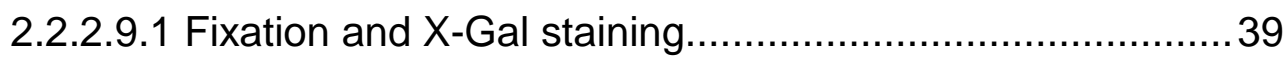

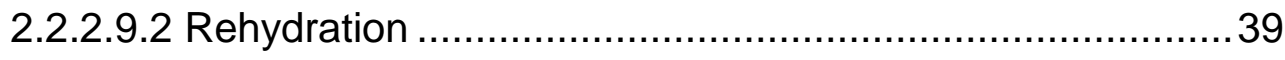

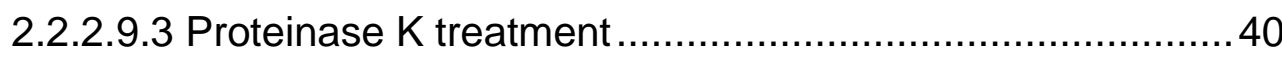

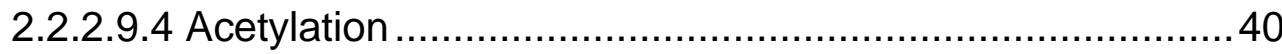

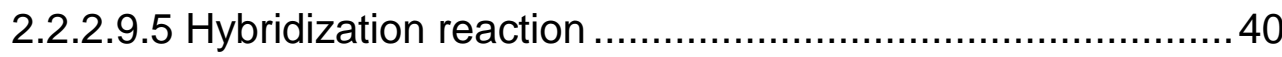

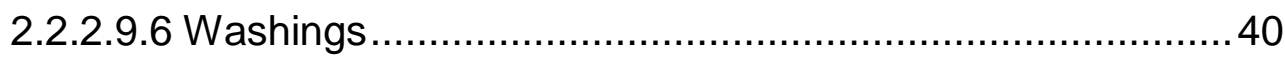

2.2.2.9.7 Antibody reaction ........................................................ 41

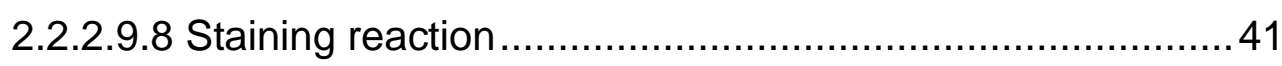

2.2.2.9.9 Background removal and bleaching ……....................... 41

2.2.3 Agarose gel electrophoresis.................................................... 41

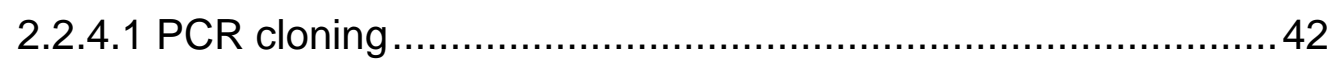


2.2.4.2 Gel purification of PCR and restriction fragments ................ 42

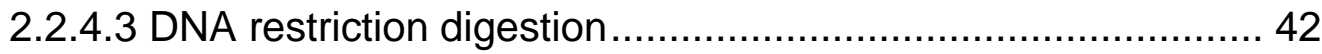

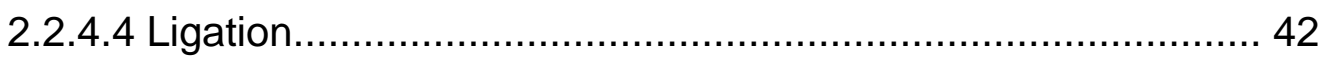

2.2.4.5 Chemical transformation of bacterial cells............................ 43

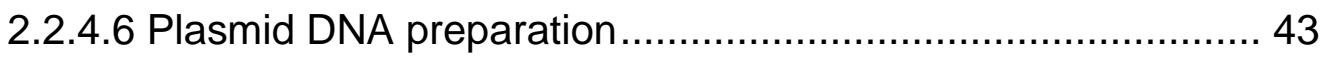

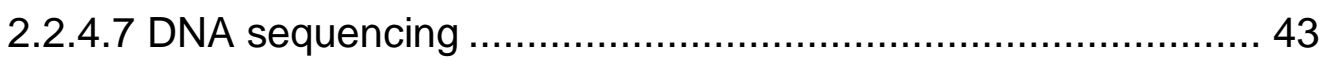

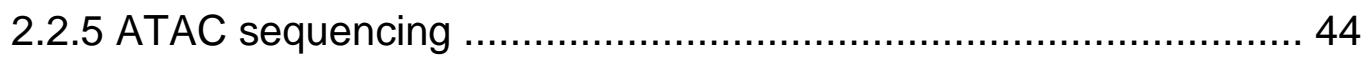

2.2.5.1 Transposition reaction .......................................................... 44

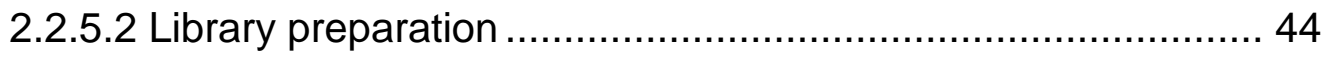

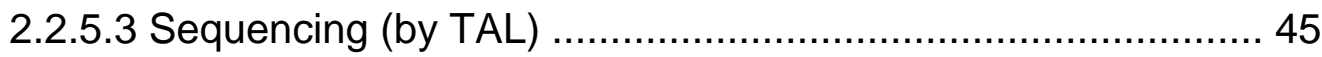

2.2.5.4 Sequencing alignment and processing (performed by $T A L$ )... 45

2.2.6 Gene expression analysis by semiquantitative RT-PCR or quantitative Nanostring analysis. 46

2.2.6.1 Total RNA isolation from ectodermal explants and whole embryos 46

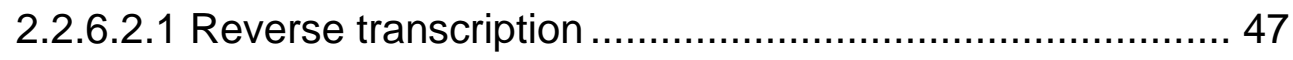

2.2.6.2.2 Reverse transcription-PCR (RT-PCR) .......................... 47

2.2.6.3 Quantitative Nanostring analysis ........................................ 47

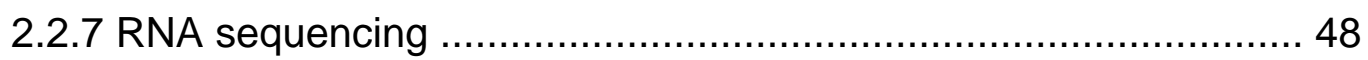

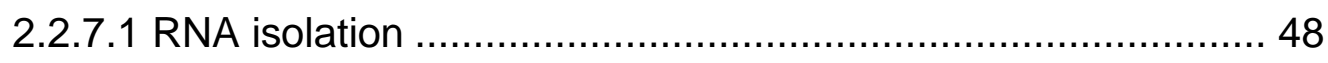

2.2.7.2 Sample preparation and sequencing..................................... 49

2.2.7.3 Sequencing alignment (performed by $\mathrm{TAL}$ ) .......................... 49

2.2.7.4 Statistical analysis (performed by TAL) ............................... 49

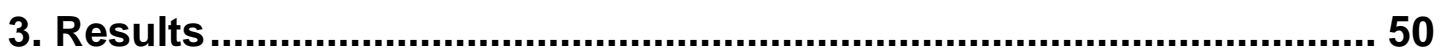

3.1 Mutation of a single threonine residue in the Ptf1a C2 domain is sufficient to induce a mixed induced neuronal transmitter phenotype

3.2 Introducing a negative charge at the $\mathrm{Ptf1aT}_{243}$ residue induces a more wildtype Ptf1a like phenotype 
3.3 The mixed neuronal transmitter phenotype induced by Ptf1a ${ }^{\mathrm{T243A}}$ is not the result of an impaired interaction with $\mathbf{R b p j}$. .57

3.4 Ptf1aT243 mutants strongly bind to Prdm13 .58

3.5 Ptf1a induces direct target genes at different time points. 62

3.6 Temporal expression of Ptf1a target genes by RNA sequencing . 65

3.7 Ptf1a target genes are highly enriched for playing roles during transcription and neurogenesis

3.8 A knock-down of Brg1 affects the induction of indirect Ptf1a target genes 69

3.9 Induction of selected Ptf1a downstream genes requires Brg1 .....71

3.10 Ptf1a can alter the chromatin state of its target genes ................75

3.11 Ptf1a target genes lay in closed chromatin regions.....................78

4. Discussion .80

4.1 The Ptf1a C-terminus is essential for the induced neuronal transmitter phenotype 80

4.2 Role of phosphorylation on Ptf1a on its activity 82

4.3 BAF complex mediated chromatin remodeling is important for the activation of delayed target gene expression by Ptf1a. 83

4.4 Ptf1a does not need open chromatin to induce its targets 85

4.5 Mechanisms driving the delayed activation of target genes ..........86

5. Bibliography. .89

6. Appendix 104

6.1 Summary of Nanostring data 104

6.1.1 Ptf1a threonine mutants 104

6.1.2 Time course analysis 106

6.1.2 Brg1 knock-down 107

6.2 Summary of the genes analyzed with the Nanostring 113

6.3 GO tables RNA sequencing experiments. 
6.3.1 RNA sequencing experiment onset of target gene activation by Ptf1a

6.3.1.1 GO analysis of candidate genes upregulated after 3,6 or $12 \mathrm{~h}$.

6.3.1.2 GO analysis of direct target genes having their onset at 3, 6 or $12 \mathrm{~h}$.

6.3.2 RNA sequencing experiment Brg1 knock-down 123

6.4 RNA-seq data 124

6.4.1 Candidate gene list for the RNA sequencing analysis of Ptf1a-GR time course 124

6.4.2 Candidate gene list for the RNA sequencing analysis of Brg1 knock-down experiment.

6.4.3 Candidate gene list for the RNA sequencing analysis of Brg1 knock-down affected genes 186

6.5 Quantification of the BiFC assay 190 


\section{Acknowledgements}

First of all, I would like to express my sincere gratitude to Dr. Kristine Henningfeld for her great supervision and endless support during this thesis. Her guidance inspired me a lot during the time of research and writing.

I would also like to thank Prof. Dr. Pieler for his help and support in many ways including fruitful discussions and suggestions. I am additionally grateful for him being a member of my examination board.

Furthermore, I would like to acknowledge my thesis committee members Prof. Dr. Johnsen, and Prof. Dr. Dresbach for sharing their valuable comments and suggestions. I also would like to thank Prof. Dr. Mansouri and Prof. Dr. Bucher. for being part of my extended thesis committee.

I thank all my lab members, past and present, for your company, support, and fruitful discussions: Especially, I would like to thank Dr. Marie Hedderich, Dr. Patrick Berndt, Dr. Maja Gere, Dr. Juliane Melchert, Dr. Barbara Rust, Esther Essel and Katja Ditter. I also would like to thank the members of the TAL for the extensive help with sequencing.

Finally, I would like to thank my family for the tremendous support. Especially I would like to thank Yehan for her company, encouragement and support during all these years. Without her I would not have made it. 


\section{Abstract}

The bHLH transcription factor Ptf1a is crucial for the generation of a GABAergic neuronal cell fate over an excitatory glutamatergic fate. Ptf1a is a component of a heterotrimeric transcription factor complex, which in addition to Ptf1a, is comprised of a commonly expressed bHLH E-protein such as E12 and Rbpj. Interaction with Rbpj is essential for the GABAergic cell fate inducing and glutamatergic repressing activities of Ptf1a. In the absence of Rpbj binding, Ptf1a maintains its proneural activity, however, the induced neurons express marker genes indicative of a glutamatergic excitatory neuronal cell fate. In this thesis, the temporal and selective induction of target genes by Ptf1a was further analyzed as well several Ptf1a mutants characterized with respect to the neurotransmitter inducing properties using $X$. laevis as a model system.

A point mutation within the C-terminus of Ptf1a that leads to the induction of a mixed glutamatergic and GABAergic neuronal transmitter phenotype was identified and characterized $\left(\mathrm{Ptf}^{\mathrm{T} 243 \mathrm{~A}}\right)$. BiFC analysis revealed that the mixed neuronal transmitter phenotype is not due to an impairment of the interaction with Rbpj or E12, but may be due to enhanced interaction with Prdm13. The global temporal transcriptional program induced Ptf1a was also studied in pluripotent embryonic cells by RNAseq. While some Ptf1a direct target genes such as prdm13, are induced within three hours (early genes), others, including neurog2 and gad1a are induced only after $12 \mathrm{~h}$ (late genes). To gain insight if chromatin accessibility plays a role in the delayed activation, Brg1, which is one of the catalytic subunits of the chromatin remodeling BAF complex, was knocked down. Brg1 was found to be required for Ptf1a-induced neuronal differentiation as shown by the loss of neural-specific tubulin. Only a subset of late induced Ptf1a target genes required Brg1 with most Brg1 dependent target genes being indirect Ptf1a target genes. Furthermore, ATAC-seq suggests that target gene activation by Ptf1a is likely independent of chromatin accessibility. Taken together, these data suggest that Ptf1a acts as a pioneer transcription factor to activate its target genes. 


\section{List of figures}

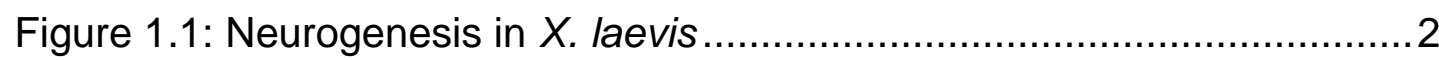

Figure 1.2: Overview over archaete-scute and atonal family members of proneural bHLH transcription factors ..........................................................

Figure 1.3: Multiple roles of the proneural bHLH transcription factor Neurog2

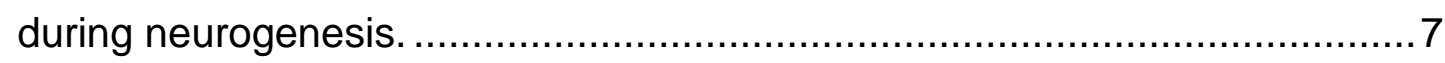

Figure 1.4: Specification of neuronal progenitors via lateral inhibition .............9

Figure 1.5 Dorsal-ventral patterning of the neural tube .............................12

Figure 1.6: Overview of epigenetic mechanisms controlling the transcription

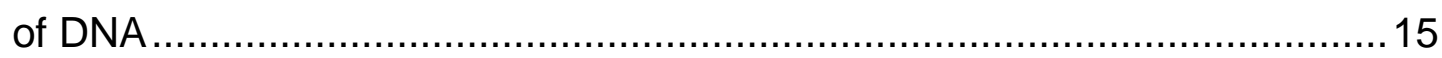

Figure 1.7 Composition of the BAF chromatin remodeling complex .............17

Figure 1.8: Expression pattern of Ptf1a ................................................21

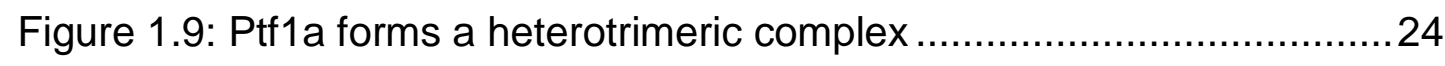

Figure 3.1: Overview $X$. laevis Ptf1a mutants ...........................................51

Figure 3.2: Ptf1 $\mathrm{a}^{\mathrm{T243A}}$ mutant induces a mixed neuronal transmitter

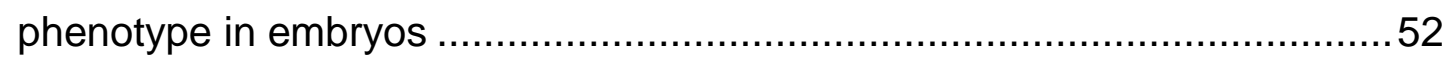

Figure 3.3 Ptf1 $\mathrm{a}^{\mathrm{T} 243 \mathrm{~A}}$ mutant induces a mixed neuronal transmitter phenotype

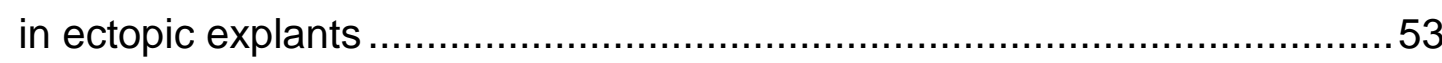

Figure 3.4: Activities of distinct Ptf1aT 243 mutants .....................................56

Figure 3.5: Ptf1 $\mathrm{a}^{\text {T243A }}$ mutation does not affect its ability to bind Rbpj ..........59

Figure 3.6: Ptf1 $\mathrm{a}^{\mathrm{T} 243 \mathrm{~A}}$ and Ptf1 $\mathrm{a}^{\mathrm{T} 243 \mathrm{E}}$ mutants strongly interact with Prdm13.

Figure 3.7: Ptf1a is activating its target genes in a temporal manner ..........64

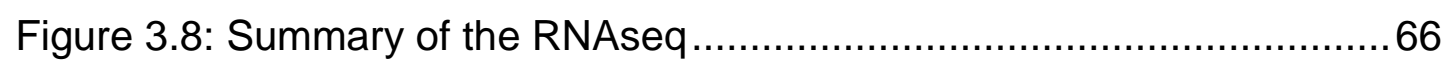

Figure 3.9 Comparison of target gene activation by Ptf1a after 3,6 and $12 \mathrm{~h}$

Figure 3.10: Genes involved in processes involved in neurogenesis are enriched in all three time points

Figure 3.11: Brg1 dependent genes are mostly indirect Ptf1a target genes. 70 Figure 3.12: Genes affected by an Brg1 knock-down have activities during neurogenesis 71

Figure 3.13: Induction of some delayed Ptf1a target genes is depended on Brg1 
Figure 3.14 Summary of the Nanostring analysis for genes induced after $3 \mathrm{~h}$ 73

Figure 3.15 Summary of the Nanostring analysis for genes induced after $3 \mathrm{~h}$ 75

Figure 3.16: Ptf1a overexpression can change the chromatin state of its target genes. 77

Figure 3.17: Chromatin remodeling plays a minor role in the delayed induction of Ptf1a target genes 79 


\section{List of tables}

Table 2.1 List of RT-primers and their PCR parameters............................29

Table 2.2 List of sequencing oligonucleotides ..........................................29

Table 2.3 List of morpholino oligonucleotides .............................................30

Table 2.4 List of overexpression constructs ............................................ 31

Table 2.5 List of RT-primers and their PCR parameters...............................35

Table 2.6 List of PCR cycles for each ATAC sample ..................................45

Table S1: Summary of average normalized counts of three independent

Nanostring experiments for each sample and gene ...............................104

Table S2: Summary of the calculated SEM of the normalized counts shown in

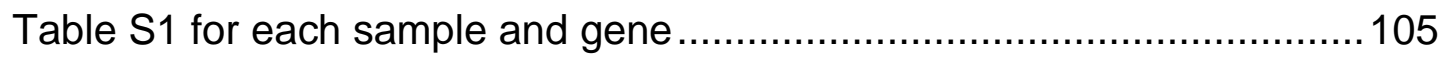

Table S3: Summary of average normalized counts of three independent

Nanostring experiments for each sample and gene …...........................106

Table S4: Summary of the calculated SEM of the normalized counts shown in

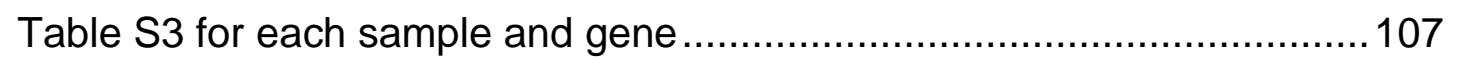

Table S5: Summary of average normalized counts of three independent

Nanostring experiments for each sample and gene …............................107

TableS6: Summary of the calculated SEM of the normalized counts shown in

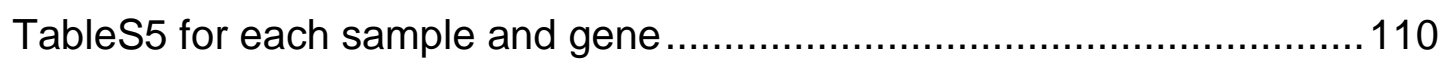

Table S7: Summary of the geneses analyzed with Nanostring....................113

Table S8: Summary of GO analysis of genes upregulated after $3 \mathrm{~h} . \ldots \ldots \ldots . . .119$

Table S9: Summary of GO analysis of genes upregulated after $6 \mathrm{~h} . \ldots \ldots \ldots . . .120$

Table S10: Summary of GO analysis of genes upregulated after $12 \mathrm{~h}$.......120

Table S11: Summary of GO analysis of direct target genes having their onset at $3 \mathrm{~h}$. 
Table S12: Summary of GO analysis of direct target genes having their onset at $6 \mathrm{~h}$. 122

Table S13: Summary of GO analysis of direct target genes having their onset at $12 \mathrm{~h}$. 122

Table S14: Summary of GO analysis Brg1 affected candidate genes. 123

Table S15: Summary of differentially expressed genes activates after $3 \mathrm{~h} 124$ Table S16: Summary of differentially expressed genes activates after 6 h 127 Table S17: Summary of differentially expressed genes activates after $12 \mathrm{~h}$

Table S18: Summary of differentially expressed genes by Ptf1a + cMO ... 147 Table S19: Summary of differentially expressed genes by Ptf1a + Brg1MO

Table S20: Summary of genes Ptf1a + cMO vs. Ptf1a + Brg1MO

(downregulated by Brg1 knock-down). 186

Table S21: Summary of genes Ptf1a + cMO vs. Ptf1a + Brg1MO

(upregulated by Brg1 knock-down) 189

TableS22: Quantification of the BiFC 190 


\section{Abbreviations}

$\begin{array}{ll}\text { \% } & \text { percent } \\ { }^{\circ} \mathrm{C} & \text { Celsius degree } \\ \text { aa } & \text { amino acid } \\ \text { alN } & \text { ascending premotor interneurons } \\ \text { BCNE center } & \text { blastula Chordin- and Noggin-expressing center } \\ \text { bHLH } & \text { basic helix-loop-helix } \\ \text { BiFC } & \text { Bimolecular Fluorescent Complementation assay } \\ \text { BMP } & \text { bone morphogenetic protein } \\ \text { bp } & \text { base pairs } \\ \text { Brg1MO } & \text { Brg1 morpholino } \\ \text { BSA } & \text { bovine serum albumin } \\ \text { CC } & \text { noninjected control caps } \\ \text { cdk } & \text { cell cycle dependent kinases } \\ \text { cDNA } & \text { complementary DNA } \\ \text { CE } & \text { noninjected control embryos } \\ \text { CHX } & \text { cycloheximide } \\ c I N & \text { commissural premotor interneurons } \\ \text { cMO } & \text { control morpholino } \\ \text { dIN } & \text { descending premotor interneurons } \\ \text { dINr } & \text { descending repetitive excitatory interneurons } \\ \text { dla } & \text { g-aminobutyric acid } \\ \text { dlc } & \text { glucocorticoid receptor } \\ \text { dII1 } & \text { dorsolateral ascending sensory interneurons } \\ \text { DNA } & \text { dorsolateral commissural sensory interneurons } \\ \text { ecIN } & \text { Notch ligand delta1 } \\ \text { EDTA } & \text { desoxyribonucleic acid } \\ \text { et al. } & \text { excitatory commissural sensory pathway interneurons } \\ \text { EtOH } & \text { ethylenediaminetetraacetic acid } \\ \text { FGF } & \text { et alii } \\ \text { for } & \text { ethanol } \\ \text { g } & \text { Fibroblast growth factors } \\ \text { GABA } & \text { forward } \\ \text { GR } & \end{array}$


h

$\mathrm{hb}$

$\mathrm{kb}$

I

LB

$\mu$

$\mathrm{m}$

M

MAB

MAPK

$\min$

$\mathrm{n}$

neurog2

NICD

$\mathrm{p}$

PBS

PCR

$\mathrm{pH}$

Ptf1a

rev

RNA

RNase

RNAseq

rpm

RT

RT-PCR

$S$

Shh

st

TAE

Tm

tp

U

V hour/hours

hindbrain

kilobase

liter

Luria Bertani

micro

milli/meter

molar

maleic acid buffer

mitogen-activated protein kinase

minutes

nano

neurogenin 2

intracellular domain of the Notch receptor

pico

phosphate buffered saline

polymerase chain reaction

negative decade logarithm of hydrogen ion concentration

Pancreatic transcription factor $1 \mathrm{a}$

reverse

ribonucleic acid

ribonuclease

RNA sequencing

rounds per minute

room temperature

reverse transcriptase PCR

seconds

Sonic hedgehog

stage

Tris-acetate-EDTA

melting temperature

trigeminal placodes

units

volume 
WMISH

X-Gal

$X$. laevis whole mount in situ hybridizations

5-bromo-4-chloro-3-indolyl- $\beta$-d-galactopyranoside

Xenopus laevis 


\section{Introduction}

\subsection{Neurogenesis in Xenopus laevis}

The African clawed frog Xenopus laevis (X. laevis) has several advantages that make it a highly suitable model system to study early vertebrate developmental events including the development of the nervous system (Pratt and Khakhalin, 2013; Kofent and Spagnoli, 2016; Borodinsky, 2017; Droz and McLaughlin, 2017; Dubey and Saint-Jeannet, 2017; Lee-Liu et al., 2017). X. laevis embryos are quite large (>1mm), undergo an external development, can easily be manipulated by microinjection of mRNA or morpholino antisense oligonucleotides as well as used for CRISPR/Cas9 mutagenesis (Mimoto and Christian, 2011; Wang et al., 2015). Furthermore, pluripotent ectodermal explants can be isolated from the animal half of the embryo at blastula stage and used for in vitro differentiation studies using embryos manipulated with the aforementioned techniques (Borchers and Pieler, 2010).

In X. laevis, there are two distinct phases of neurogenesis (Hartenstein, 1989; Roberts, 2000; Thuret et al., 2015). The first phase is termed primary neurogenesis and generates the neurons responsible for the movements and sensation of the tadpole (Hartenstein, 1989; Hartenstein, 1993; Roberts, 2000). This phase of neurogenesis starts at the open neural plate stage and slowly declines until the mid-tadpole stage, where it remains minimally active (Thuret et al., 2015). A second phase of neurogenesis occurs during metamorphosis, in which most early-born neurons are replaced generating the nervous system of the adult frog (Schlosser et al., 2002; Wullimann et al., 2005).

The first primary neurons are born within $24 \mathrm{~h}$ after fertilization, in three longitudinal domains (medial, intermediate and lateral) along the dorsal midline and will give rise to neurons of the hindbrain and spinal cord and in the trigeminal placodes (Chitnis and Kintner, 1995). In the anterior neural plate, which gives rise to the forebrain and midbrain, neuronal differentiation starts at the tailbud stage (Papalopulu and Kintner, 1996). After the neual plate rises and folds into the neural tube, the progenitors in the medial domain will reside ventrally and differentiate into motor neurons, those from the intermediate 
domain into interneurons, while the progenitors from the lateral domain at the neural plate border will be located most dorsally and differentiate into sensory neurons (Hartenstein, 1989; Chitnis and Kintner, 1995). In the X. laevis tadpole there are 10 distinct classes of neurons (Roberts et al., 2012). The first neurons to gain their functionality are the Rohon-beard sensory neurons located adjacent to the roof plate in the dorsal neural tube (Lamborghini, 1980; Rossi et al., 2009). Subsequently, the ventral motor neurons and Kolmer-Agdhur cells (Rossi et al., 2008; Rossi et al., 2009; Groves and LaBonne, 2014) and then seven subpopulations of interneurons are formed (Roberts et al., 2012) (Fig 1.1).
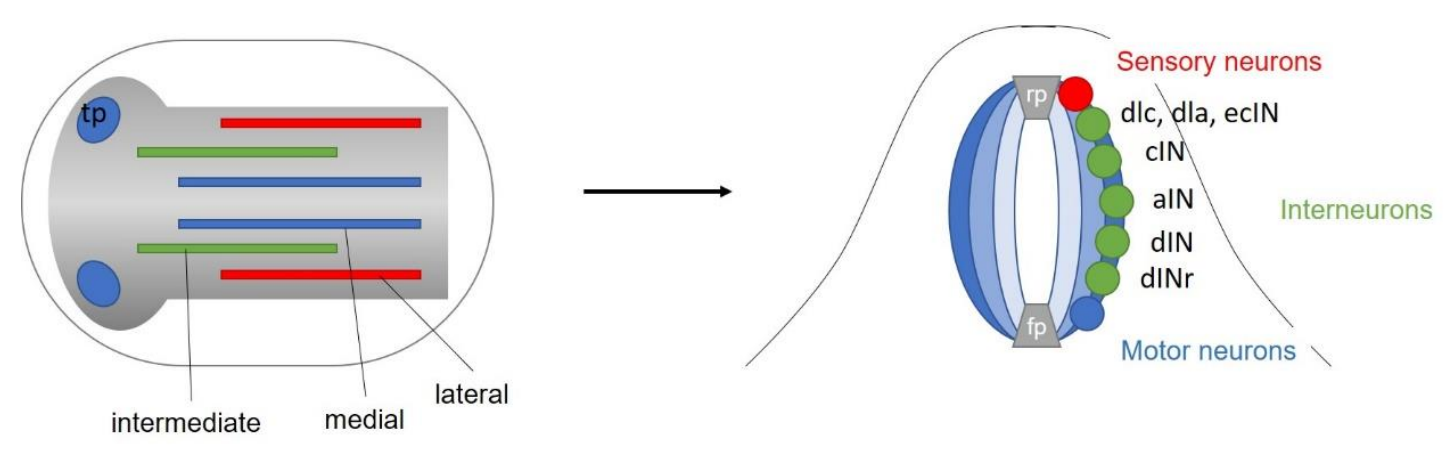

Figure 1.1: Neurogenesis in $X$. laevis

The first primary neurons are born at the open neural plate stage in three longitudinal domains along the dorsal midline. After neural tube closure, the medial domain will give rise to motor neurons in the ventral neural tube, the lateral domain will give rise to sensory neurons in the dorsal neural tube and the intermediate domain will be located medially in the neural tube and give rise to interneurons. tp: trigeminal placodes dla, dlc: dorsolateral ascending and dorsolateral commissural sensory interneurons; $d I N, c / N$, alN: descending, commissural, and ascending premotor interneurons; dINr: descending repetitive excitatory interneurons; ec/N: excitatory

\subsection{Neural induction}

The development of the nervous system is initiated during neural induction where subpopulations of the ectoderm are specified to a neural fate (Rogers et al., 2009). In X. laevis, the neural fate is induced during gastrulation (Hamburger, 1969; Sanes et al., 2011) and requires the inhibition of BMP signaling (Hemmati-Brivanlou et al., 1994; Hemmati-Brivanlou and Melton, 1994; De Robertis and Kuroda, 2004). The inhibition of BMP signaling occurs through multiple mechanisms, which includes the secretion of BMP 
antagonists from the blastula Chordin- and Noggin-expressing (BCNE) center, which is located in dorsal animal cells at the blastula stage (Kuroda et al., 2004) and during gastrulation from the Spemann Organizer through Chordin (Piccolo et al., 1996; Sasai et al., 1996), Noggin (Zimmerman et al., 1996), Follistatin (Hemmati-Brivanlou et al., 1994) and Cerberus (Bouwmeester et al., 1996; Piccolo et al., 1999). In addition to BMP signaling, a requirement for FGF signaling in the process of neural induction has been established (Launay et al., 1996; Sasai et al., 1996). FGF signaling results in MAPK-induced phosphorylation of Smad1, the intracellular transducer of BMP signaling, resulting in an inhibition of BMP activity (Hardcastle et al., 2000; Pera et al., 2001; Richard-Parpaillon et al., 2002; Pera et al., 2003). In addition to its role in inhibiting BMP, FGF is required for the induction and maintenance of several neural genes (Rogers et al., 2011). Furthermore, $\mathrm{Ca}^{2+}$ transients are present in the dorsal ectoderm during neural induction and blocking of calcium release prevents the expression of neuronal markers (Leclerc et al., 1997; Leclerc et al., 2000; Leclerc et al., 2012).

Neural induction leads to the expression of several genes in the forming neural plate including members of the Sox family (sox2, sox3 and sox 15 , formally known as soxD), Zic family (zic1-3) and Iroquois family (iro1-3), as well as the forkhead box protein foxD5 and geminin (Nakata et al., 1997; Bellefroid et al., 1998; Brewster et al., 1998; Gomez-Skarmeta et al., 1998; Kroll et al., 1998; Mizuseki et al., 1998; Mizuseki et al., 1998; Sölter et al., 1999; Uchikawa et al., 1999; Sullivan et al., 2001; Graham et al., 2003; Penzel et al., 2003; Pevny and Placzek, 2005; Pitulescu et al., 2005; Seo and Kroll, 2006; Kroll, 2007; Lefebvre et al., 2007; Houtmeyers et al., 2013).

The genes induced by neural induction regulate multiple processes including progenitor maintenance (sox2, sox3 and zic2) (Brewster et al., 1998; Hardcastle et al., 2000; Ellis et al., 2004; Pevny and Placzek, 2005; Rogers et al., 2009), the onset of neuronal differentiation (zic1, zic3, sox15, iro1, iro2 and iro3) (Nakata et al., 1997; Mizuseki et al., 1998; Rogers et al., 2009) and control of progenitor proliferation (geminin, foxD5) (Kroll et al., 1998; Sullivan et al., 2001; Pitulescu et al., 2005; Seo and Kroll, 2006; Kroll, 2007; Papanayotou et al., 2008). 


\section{3 bHLH transcription factors and their role during neuronal differentiation and determination}

Transcription factors of the basic helix-loop-helix (bHLH) transcription factors are involved in many developmental processes including muscle development (Weintraub, 1993), mesodermal determination (Burgess et al., 1995), skeletal development (Cserjesi et al., 1995) and neural development (Lee, 1997; Bertrand et al., 2002). Members of the bHLH transcription factor family can be categorized into three classes (Murre et al., 1989). Class A bHLH proteins form homo or heterodimers and are ubiquitously expressed genes such as E12, E47 or daughterless. Class B bHLH transcription factors form heterodimers with class $\mathrm{A}$ bHLH proteins and show a tissue specific expression pattern, while class $\mathrm{C}$ bHLH transcription factors do not interact with either of the class A or class B bHLH transcription factors (Murre et al., 1989). The formed homoor heterodimers bind to an E-box motif (CANNTG) on the DNA, usually through the basic region, while the $\mathrm{HLH}$ domain is involved in the dimerization (Murre et al., 1989). The activity and DNA target choice of bHLH transcription factors can be regulated via the interaction with co-factors (Ma et al., 2008; Mattar et al., 2008; Rodolosse et al., 2009; Li et al., 2011).

Neuronal differentiation is initiated by the expression of proneural genes, which drive the determination and differentiation of progenitor cells to a neuronal cell fate (Bertrand et al., 2002). The proneural genes are mainly class B bHLH transcription factors (Bertrand et al., 2002) belonging to the drosophila archaete-scute and atonal family. Proneural bHLH transcription factors can be categorized in two distinct subgroups according to their function during neuronal differentiation and based on the timing of their expression: neuronal determination genes and neuronal differentiation genes (Lee, 1997; Farah et al., 2000; Bertrand et al., 2002). Neuronal determination genes such as neurog2 or ascl1 are expressed early during neurogenesis in the mitotically active progenitors, which in the neural tube, are found in the inner ventricular zone (Lee, 1997; Bertrand et al., 2002). Neuronal differentiation genes such as neurod 1 are expressed slightly later compared to the determination genes and are positively regulated by the proneural determination genes (Lee, 1997; Bertrand et al., 2002). During neuronal differentiation, bHLH transcription are 
involved in the regulation of several processes (Fig.1.3), which in the following will be demonstrated by the multiple activities of the proneural bHLH transcription factor Neurog2.

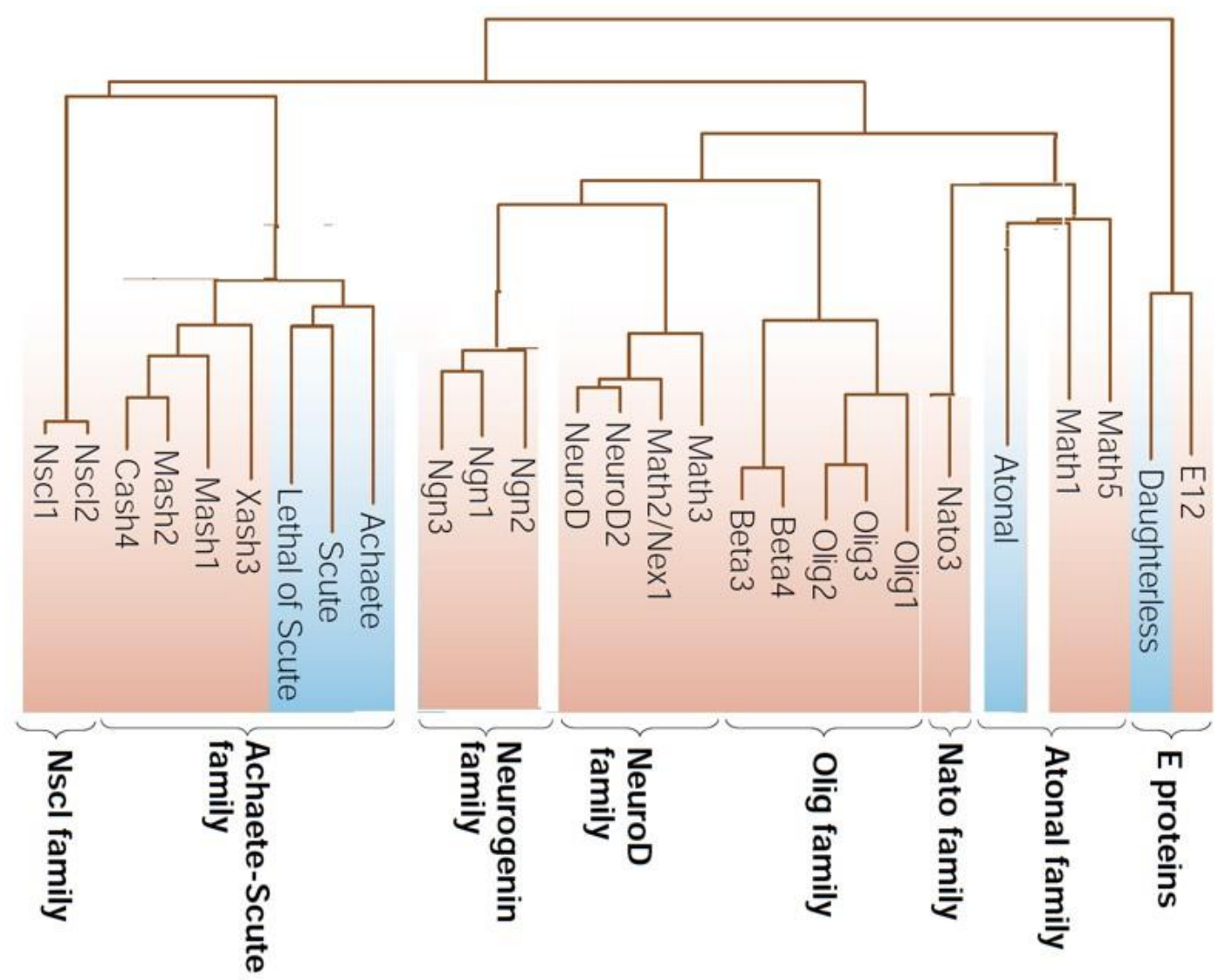

Figure 1.2: Overview over Archaete-Scute and Atonal family members of proneural bHLH transcription factors

Scheme showing the relationship between different families of bHLH proteins involved in neuronal differentiation. bHLH proteins are mainly divided in ubiquitously expressed E-proteins, which are orthologs to drosophila Daughterless and the proneural families that are homologue to the drosophila Atonal and Achaete-Scute. The Atonal family can be further divided in several subfamilies, which includes the Neurogenin family, the Olig family and the Atonal family. Blue shading highlights the invertebrate family members, while red highlights vertebrate family members. (Modified from Bertrand et al. (2002).

Neurog2 is a member Neurogenin family of the atonal-like bHLH transcription factors. This family consists of three distinct members (Neurog1, Neurog2 and Neurog3) (Sommer et al., 1996; Nieber et al., 2009), all of which are expressed during gastrulation in $X$. laevis. (Nieber et al., 2009). Transcripts of neurog1 and neurog2 are present in the three longitudinal domains of primary neurogenesis in open neural plate stages, with the expression of neurog2 
being broader than neurog1 (Ma et al., 1996; Nieber et al., 2009). Neurog3 expression, in comparison, is restricted to the medial domain (Nieber et al., 2009).

Among the members of the Neurogenin family, the function of neurog 2 is best characterized in $X$. laevis. Overexpression of neurog 2 is sufficient to drive neuronal differentiation of the non-neural ectoderm (Ma et al., 1996). Furthermore, Neurog2 induces the expression of other later expressed proneural bHLH transcription factors such as neurod1, which on the other hand, is not capable of inducing neurog2 (Ma et al., 1996). Other early expressed bHLH transcription factors such as Ascl1, which activates neurod1 expression (Cau et al., 1997), while Neurod1 levels do not affect asc/1 expression (Gao et al., 2009).

It has been demonstrated that in mammalian cell lines, Neurog2 can act as a pioneer transcription factor, initiating target gene transcription on nucleosome bound regions (Chen and Dent, 2014). Often the binding of a pioneer transcription factor will initiate events that lead to an opening of the chromatin and the recruitment of active histone marks stabilizing target gene transcription (Zaret and Carroll, 2011; Zaret and Mango, 2016). Neurog2, for example, binds to regions of non-accessible chromatin (Smith et al., 2016) and promotes the removal of repressive histone marks and the addition of active histone marks at the neurod 1 and tubb2b promoters by interacting with the $\mathrm{H} 3$ lysine 9 demethylase Kdm3a (Lin et al., 2017). Several other proneural bHLH transcription factors such as Ascl1 or Neurod1 have been demonstrated to act as pioneer transcription factors in murine ES cells and fibroblasts (Wapinski et al., 2013; Pataskar et al., 2016), indicating a general role of proneural bHLH transcription factors in initiating and stabilizing the onset of neuronal gene expression. 


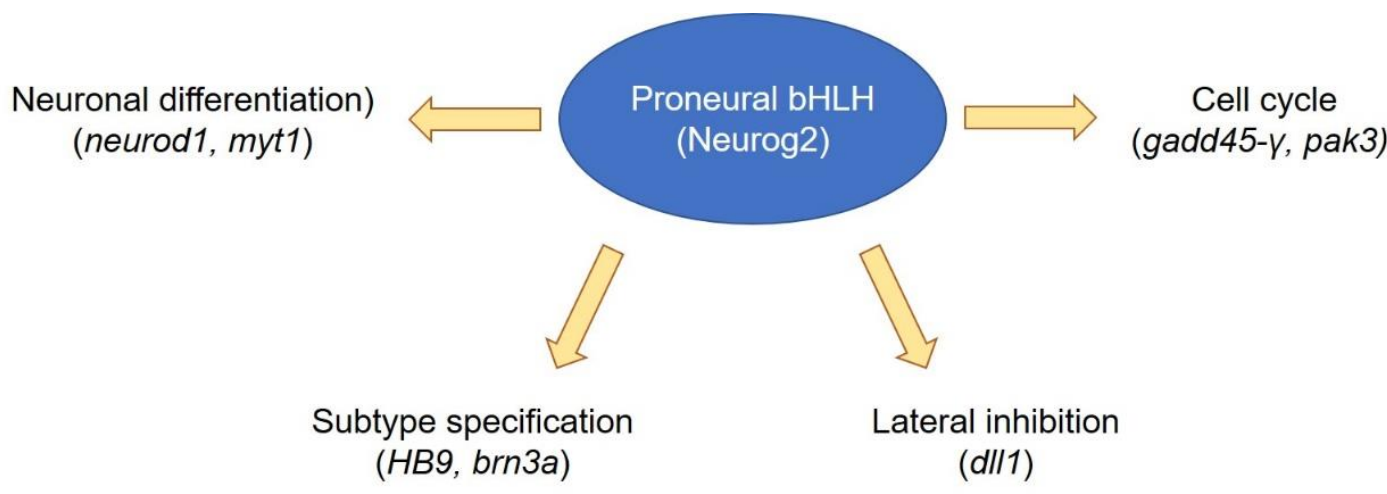

Figure 1.3: Multiple roles of the proneural bHLH transcription factor Neurog2 during neurogenesis. bHLH transcription factors like Neurog2 are involved during many processed in regulating neurogenesis. Neurog2, for example is activating a cascade of proneural genes driving neuronal determination and differentiation (Bellefroid et al., 1996; Ma et al., 1996). It is furthermore involved in activating cell cycle regulators and Notch ligand dIII and thereby controls the cell cycle length and lateral inhibition (de la Calle-Mustienes et al., 2002; Kiyota and Kinoshita, 2002; Souopgui et al., 2002; Bray, 2006). In addition, Neurog2 activates genes involved in the specification of distinct neuronal subtypes (Bertrand et al., 2002).

The proneural bHLH proteins lead to the transcription of other bHLH transcription factors that are involved in the differentiation to neuronal cells, such as the HLH transcription factors ebf2 (Dubois et al., 1998) and ebf3 (Pozzoli et al., 2001), the atonal family members atoh1 (Kim et al., 1997) and atoh7 (Kanekar et al., 1997), as well as the neuronal differentiation factors neurod1 (Ma et al., 1996) and neurod4 (Perron et al., 1999). For example, the overexpression of the bHLH transcription factor Atoh1 is sufficient to program ectodermal cells to express neuronal differentiation markers in $X$. laevis without inducing early neuronal differentiation marker genes such as neurog2 or neurod1 (Kim et al., 1997).

Besides driving neuronal differentiation, proneural bHLH transcription factors also influence the length of the cell cycle. Neurog2 indirectly represses cyclins D, E1 and E2 involved in G1-phase progression and G/S-phase transit in chick embryos (Lacomme et al., 2012; Pfeuty, 2015). Furthermore, Neurog2 induces the cell cycle inhibitor genes gadd45- $y$ and pak3 in $X$. laevis, leading to cell cycle withdrawal (de la Calle-Mustienes et al., 2002; Souopgui et al., 2002). The involvement of proneural bHLH proteins in regulating the cell cycle is further supported by studies in mouse, Ascl1 activates several cell cycle regulators that are essential for G1/S transition (e2f1, cdk1, cdk2, and skp2) or entry into mitosis (cdk1 and cdc25b) (Castro et al., 2006; Castro et al., 2011), 
but also other factors associated with cell cycle arrest (fbxw7, gadd $45 g$, ccng2h, and prmt2), indicating that Ascl1 has a role in regulating progenitor proliferation as well as in cell cycle exit of progenitor cells (Castro et al., 2011).

Furthermore, many bHLH transcription factors are involved in the regulation of lateral inhibition mediated by the Notch pathway, which controls the number of cells that undergo neuronal differentiation (Lewis, 1998; Bray, 2006; Kageyama et al., 2008; Ahnfelt-Rønne et al., 2012).

\subsection{Lateral inhibition}

The decision as to which cells will differentiate to functional neurons from an equivalent population of cells is under control of lateral inhibition, which is mediated by the Notch signaling pathway (Beatus and Lendahl, 1998; Bray, 2006; Kageyama et al., 2008) (Fig. 1.4A).

During $X$. laevis neuronal differentiation, the expression of Neurog2 and the achaete-scute family member Ascl1 leads to the induction of the Notch-ligands dII1 and dII4 and jagged (Kiyota and Kinoshita, 2002; Bray, 2006) (Fig. 1.4B). The Notch ligands are transmembrane proteins that recognize and bind to Notch transmembrane receptors on the neighboring cell (Chitnis and Kintner, 1995; Bray, 2006). This leads to several cleavage events on the Notch receptor, which results in the release of intracellular domain of the Notch receptor (NICD) (Schroeter et al., 1998; Selkoe and Kopan, 2003) (Fig 1.4b). The NICD then translocates to the nucleus where it interacts with CSL DNA binding protein Rbpj and induces the expression of bHLH transcription factors of the hairy and enhancer of split (hes) family (Sasai et al., 1992; Wettstein et al., 1997; Ohtsuka et al., 1999; Davis and Turner, 2001; Bray, 2006). The hes genes are transcriptional repressors of proneural genes such as neurog2, so that in the end the expression of $d l l 1$ in the neighboring cell is inhibited as well as the activation of downstream differentiation factors (Dawson et al., 1995; Wettstein et al., 1997; Li and Baker, 2001; Schneider et al., 2001; Cau et al., 2002; Louvi and Artavanis-Tsakonas, 2006) (Fig 1.4B). This leads to the differentiation of the dll1 expressing cell, while the Notch expressing cell will remain 
undifferentiated and can differentiate at a later time into a neuron or a glial cell (Sasai et al., 1992; Ohtsuka et al., 1999; Davis and Turner, 2001).

A

Neural progenitors

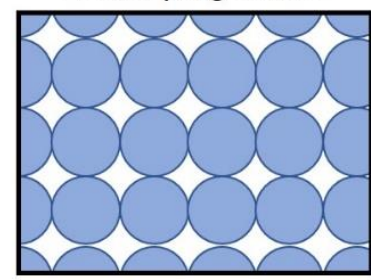

B

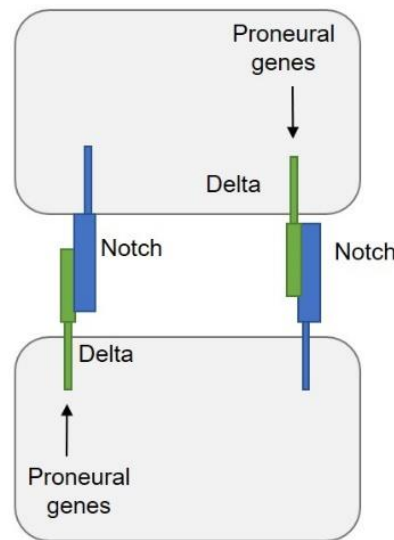

Lateral inhibition
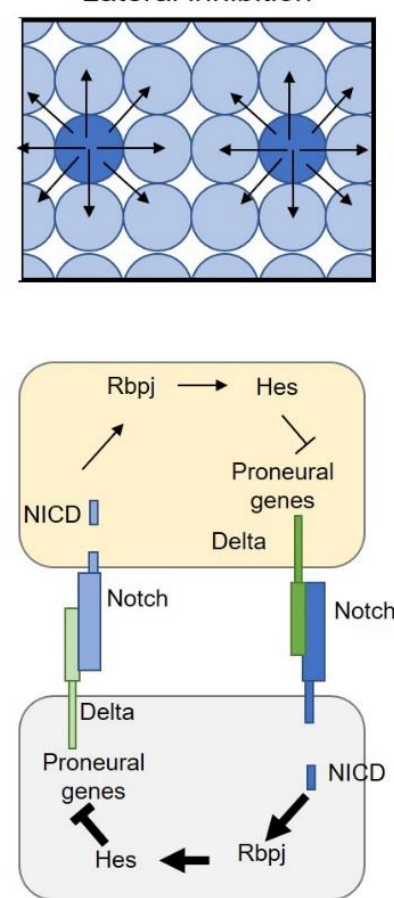

Neuronal precursors
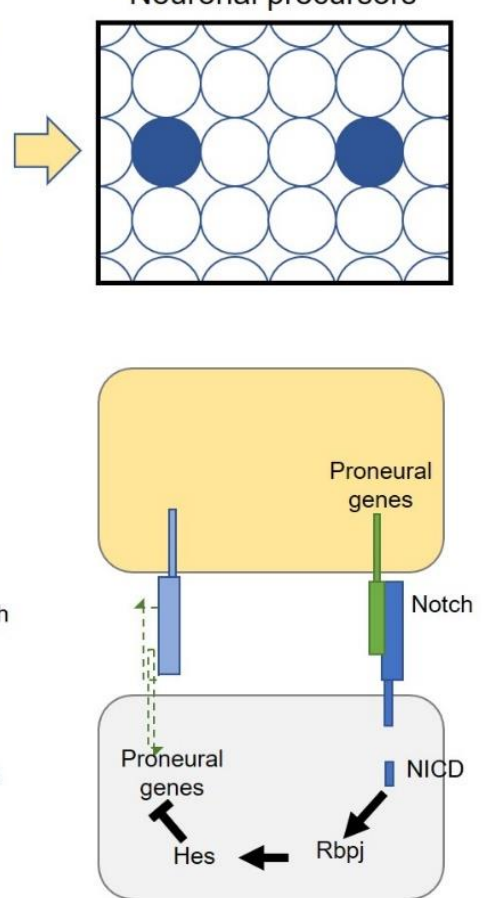

Figure 1.4: Specification of neuronal progenitors via lateral inhibition.

(A) After neural induction, the neural plate consists of equipotent neural progenitor cells. During lateral inhibition, cells get specified by signaling of repressive signals to their neighboring cells, leading signaling cells becoming neuronal progenitor cells, while receiving cells remain undifferentiated and will later give rise to stem cells or glial cells. (B) Overview of the Notch signaling pathway. Expression of proneural genes leads to the expression of the Notch ligands such as delta, which binds to the transmembrane Notch receptor on the neighboring cell. The binding of the ligand to the receptor leads to cleavage events at the Notch receptor, so that the notch intracellular domain, NICD, will be released. NICD enters the nucleus and binds to Rbpj activating repressor bHLH proteins such as the hes genes. The Hes proteins in turn, suppress the expression of proneural genes and so to a reduction of delta production in the receiving cell. In the end, the cell receiving the smallest amount of repressing signals will differentiate further.

Overexpression of NICD in $X$. laevis embryos leads to an inhibition of neuronal differentiation, while the overexpression of a dominant negative dll1 increases the number of neurons in the territories of primary neurogenesis (Chitnis and Kintner, 1995). These findings demonstrate the importance of lateral inhibition in the balance between proliferation and differentiation, In X. laevis, Neurog2 also activates the zinc finger gene myt1 (Bellefroid et al., 1996), which allows the cell to escape lateral inhibition (Bellefroid et al., 1996). After neural tube 
closure, Notch signaling remains important for preserving a neural progenitor pool in the ventricular zone of mouse and rat embryos (Lindsell et al., 1996; Imayoshi and Kageyama, 2011).

It has been demonstrated in mouse and zebrafish that the expression levels of proneural genes are oscillating within the cell, thereby establishing a dosedependent regulation of neuronal differentiation mediated by lateral inhibition (Horikawa et al., 2006; Kageyama et al., 2008; Shimojo et al., 2008; Oginuma et al., 2010; Niwa et al., 2011; Okubo et al., 2012; Roese-Koerner et al., 2017). The expression levels of the bHLH repressor hes 1 are inversely oscillating with those of other proneural genes like neurog2, ascl1 and dll1 in a 2-3 h period (Ohtsuka et al., 1999; Kageyama et al., 2008; Shimojo et al., 2008; Maurer et al., 2014; Pfeuty, 2015). Hes1 oscillation itself is established by a double negative feedback loop with the miRNA-9 (miR-9), which leads to the degradation of hes 1 mRNA, while the Hes 1 protein inhibits miR-9 expression (Bonev et al., 2012; Roese-Koerner et al., 2017). Furthermore, miR-9 also directly binds to the NICD-Rbpj transcription factor complex leading to attenuation of Notch-signaling and promotion of neuronal differentiation (Roese-Koerner et al., 2016; Roese-Koerner et al., 2017). The importance of gene oscillation is supported by the findings that continuous expression of hes 1 and dll1 lead to defects during neurogenesis (Shimojo et al., 2008; Shimojo et al., 2016).

\subsection{Neuronal subtype specification}

During neurulation, the identity of the distinct neurons is defined by their positioning in the closing neural tube (Bertrand et al., 2002; Hori and Hoshino, 2012; Roberts et al., 2012). Depending on their position, the differentiating neuronal precursor cell will receive different signals, leading to its development to a certain neuronal subtype identity (Bertrand et al., 2002). In X. laevis, ten distinct neuronal subpopulations exist, which are the Rohon-Beard sensory neurons, the ventral motor neurons and Kolmer-Agduhr cells, and seven distinct interneuronal subtypes (Roberts et al., 2012). How the different neuronal subpopulations are defined in $X$. laevis is not well established. 
However, the combinatorial of transcription factors for each progenitor domain and post-mitotic neuron has been well characterized in mice and chick embryos, and for those genes studied in $X$. laevis, a conservation of function and expression is preserved.

In contrast to $X$. laevis where the first primary neurons arise already at the open neural plate stage, in mouse and chick, the first neurons are born in the progenitor domains of the closed neural tube (Aaku-Saraste et al., 1996). The neural tube is patterned by the secretion of two morphogens at the dorsal and ventral side (Le Dréau and Martí, 2012). On the dorsal side, the neural ectoderm and the roof plate secrete BMP, while the notochord secretes Sonic hedgehog (Shh), which induces the overlying floor plate to secrete Shh to the ventral neural tube (Jessell and Dodd, 1990; Liem et al., 1997). Through the different dosages of Shh and BMP signaling, the neural tube is patterned to give rise to eleven progenitor domains of neuronal subtypes (Hori and Hoshino, 2012).

The different dosages of Shh and BMP signaling lead to the expression of domain specific combinations of bHLH and homeodomain transcription factors (Briscoe et al., 2000; Briscoe and Ericson, 2001; Helms and Johnson, 2003; Hernandez-Miranda et al., 2017). For example, Ascl1 is expressed in populations that will later develop into dl3-6 and dIL interneurons (Gross et al., 2002; Müller et al., 2002), while cells expression Neurog1 and 2 will give rise to dl2 interneurons (Gowan et al., 2001).

The progenitor domains can all be characterized by giving rise to excitatory or inhibitory neuronal subpopulations. Most excitatory neurons will secrete glutamate as a neurotransmitter, while inhibitory neurons secrete $\mathrm{y}^{-}$ aminobutyric acid (GABA) or glycine and are involved in balancing the excitatory signals (Hori and Hoshino, 2012). In the mouse dorsal neural tube, six progenitor domains are present (Helms and Johnson, 2003), which give rise to six early (dl1-6) and two late (dILA and dILB) subpopulations of interneurons. The five ventral progenitor domains give rise to five early developing classes of neurons (V0-3 and VMN). The V0 and V2 domain will further develop into two subpopulations expressing different neuronal transmitters (Hori and Hoshino, 2012) (Fig. 1.4). 

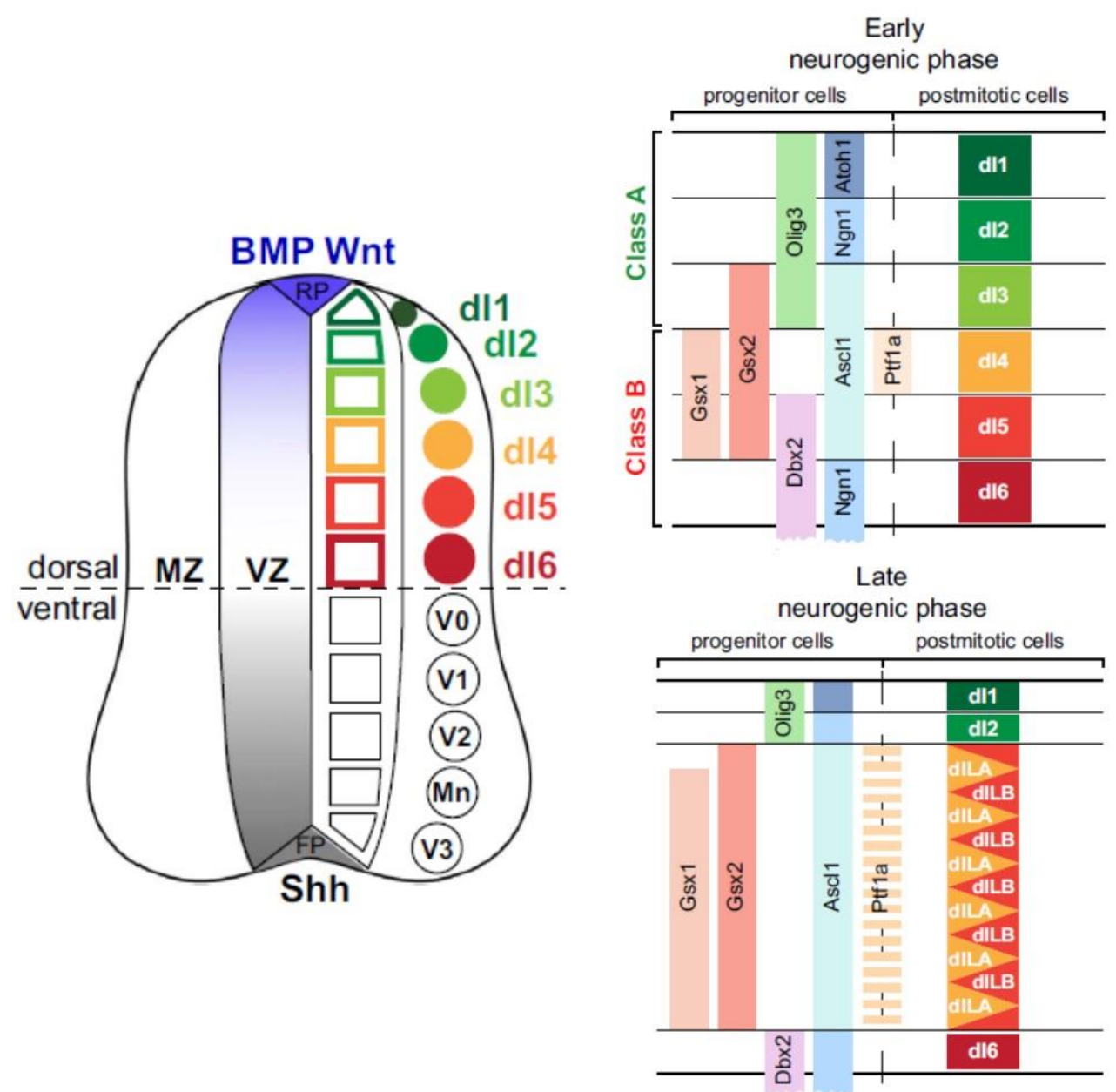

Figure 1.5 Dorsal-ventral patterning of the neural tube

In mice, the neural tube gives rise to six early and two late dorsal population of neurons (dl1-6, dILA/B) and five ventral neurons (V0-V3, MN). The identity of the distinct neuronal progenitor populations is defined by expressing a unique combination of transcription factors, which is established by gradients of Shh and BMP signaling form the dorsal and ventral neural tube (Hernandez-Miranda et al., 2017).

Interestingly, many bHLH transcription factors involved in converting the ectoderm to a general neuronal fate also drive the development of the induced neurons to a distinct neuronal subtype (Bertrand et al., 2002). Moreover, regulation of subtype specification by bHLH transcription factors is highly regulated by co-factor interaction. For instance, in the mouse, Neurog1 and Neurog2, induce the expression of sensory neuronal marker genes (Fode et al., 2000). Overexpression of Neurog2 in chick embryos together with the bHLH transcription factor Olig2, however, induces motor neuron cell fate (Mizuguchi et al., 2001), while overexpression of Neurog2 alone induces the 
expression of the glutamatergic sensory marker gene $t / x 3$ (Patterson and Krieg, 1999; Perron et al., 1999). In contrast, the progenitors with expression of Mash1 undergo a GABAergic cell fate in mice (Fode et al., 2000; Parras et al., 2002), but Mash1 together with Phox2b induces the expression of phox2a leading to the development of noradrenergic neurons (Pattyn et al., 2000).

\subsection{Role of phosphorylation in regulating neurogenesis}

Impairments during neurogenesis are associated with severe diseases including epilepsies, amyotrophic lateral sclerosis or even to agenesis of entire brain areas (Sellick et al., 2004; Allain et al., 2011; Poduri and Lowenstein, 2011). Thus, strict regulation of neurogenesis is essential for the development of a functional nervous system. It is therefore not surprising that most aspects of neurogenesis are under a high degree of regulation ranging from transcriptional, post-transcriptional and to changes on the epigenome (Ubersax and Ferrell, 2006; Yao and Jin, 2014; Hsieh and Zhao, 2016; Yao et al., 2016).

Since cells differentiate after they leave the cell cycle, the timing of cell cycle exit and length of the cell cycle is essential for the balance between neural progenitor cell maintenance and differentiation (Lange et al., 2009; Lange and Calegari, 2010; Miyata et al., 2010; Hardwick et al., 2015). Cell cycle is mainly under the control of cell cycle dependent kinases (Cdk) that transfer a phosphate group to serine or threonine residues of target proteins and in this way regulates their activity (Vernon, 2003; Richard-Parpaillon et al., 2004). Serine or threonine residues, that are modified by Cdk phosphorylation, are often directly followed by a proline residue (SP or TP sites) (Ubersax and Ferrell, 2006).

The importance of cell-cycle-dependent-phosphorylation in neuronal differentiation is supported by the findings that many proneural genes such as Neurog2 or Neurod1 are phosphorylated at several S/T-P residues (Ali et al., 2011; Hardwick and Philpott, 2015). Cell-cycle-dependent-phosphorylation on SP sites in Neurog2 negatively affects its stability and thus decreases its 
activity in inducing neuronal differentiation (Vosper et al., 2007; Ali et al., 2011). Furthermore, overexpressing of Neuorg2, where all the serine residues at SP sites were mutated to alanine in $X$. laevis embryos, showed an increase in protein stability (Ali et al., 2011). Corresponding with this increased stability comes an increased activity compared to the wild-type Neurog2 in promoting neuronal differentiation (Ali et al., 2011).

On the other hand, it has been demonstrated that phosphorylation on a conserved serine residue within the HLH domain of Neurog2 and Ascl1 regulates the selectivity of target gene activation during neuronal subtype specification in drosophila and mouse (Quan et al., 2016). Furthermore, Neurog2 phosphorylation at the C-terminus (S231and S234) has a direct influence on the interaction with binding partners during neuronal subtype specification as it facilitates the interaction with LIM homeodomain transcription factors Lhx3 and Is/1/2 during murine motor neuron specification (Ma et al., 2008).

\subsection{Epigenetic regulation of neurogenesis}

The influence of epigenetic regulation of gene expression has drawn increased attention in the last years, as malfunctions in epigenetic regulation have been associated with the development of severe diseases including several types on cancer (Hsieh and Zhao, 2016). Epigenetic mechanisms also play a critical role in neural development and function, with epigenetic dysfunction associated with several neurological disorders (Banik et al., 2017; DelgadoMorales et al., 2017). Gene transcription can be affected through multiple epigenetic events including DNA methylation, regulation via miRNAs or ncRNAs, chromatin remodeling and the modification of histone proteins (Fig. 1.6A) (Hsieh and Zhao, 2016).

Chromatin is a nucleoprotein complex of $147 \mathrm{bp}$ of DNA surrounding a core particle (nucleosomes). One nucleosome core particle consists of DNA wrapped around two copies of the histone proteins $\mathrm{H} 2 \mathrm{~A}, \mathrm{H} 2 \mathrm{~B}, \mathrm{H} 3$ and $\mathrm{H} 4$ (Luger and Richmond, 1998). Depending on the density of the nucleosomes, transcription of a target gene can be facilitated or hindered. Relatively open 
accessible chromatin regions are referred to as euchromatin, while those chromatin regions, in which the histone proteins are densely packed and the accessibility of the DNA is reduced is referred to as heterochromatin (Gao, 2017).

A

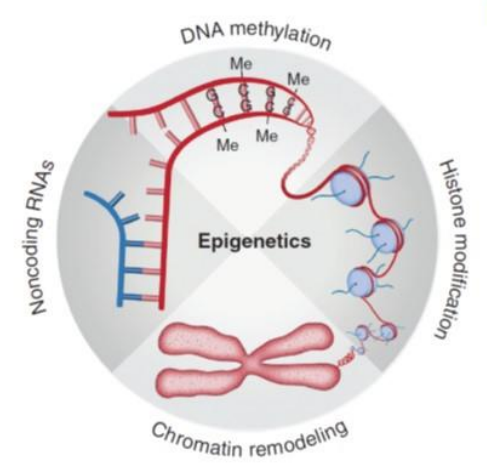

B Active Chromatin

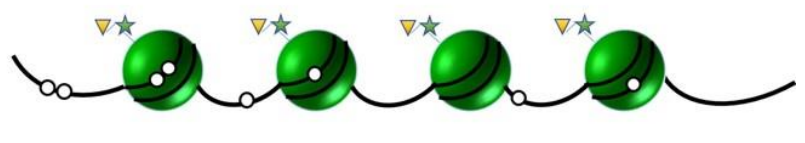

Inactive Chromatin

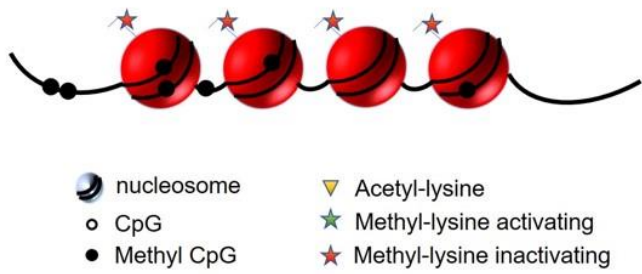

Figure 1.6: Overview of epigenetic mechanisms controlling the transcription of DNA

(A) DNA transcription can be affected by four primary mechanisms: Through ncRNAs, DNA methylation, histone modification and chromatin remodeling (Hsieh and Zhao, 2016) (B) Overview of active and inactive chromatin: Active chromatin is defined by activating histone marks such as acetylation or activating methylation on lysine residues. Inactive chromatin is characterized by repressive histone marks such as inactivating methylation on lysine residues or by methylated DNA.

The tails of histone proteins are target for covalent modifications such as acetylation, methylation, ubiquitination, SUMOylation, ribosylation and phosphorylation (Jenuwein and Allis, 2001; Bernstein et al., 2007). Depending on the type and site of modification, a modification can be referred to as activating or repressing. In general, acetylation and phosphorylation of residues is associated with the activation of transcription (Strahl and Allis, 2000), while for histone methylation, it depends on the modified residue and the number of methyl groups that are transferred to this residue to define if the modification is activating or repressing (Mosammaparast and Shi, 2010) (Fig. 1.6B). Examples for activating histone modifications are the acetylation of the lysine 9 or the lysine 14 of histone H3 (H3K9ac, H3K14ac), the di- or trimethylation of lysine 4 of histone $\mathrm{H} 3$ (H3K4me2 H3K4me3) or the phosphorylation of serine 10 of histone H3 (H3S10p) (Hsieh and Zhao, 2016). Examples for repressing marks are the demethylation of lysine 9 of histone $\mathrm{H} 3$ 
(H3K9me2) or di- and trimethylation of lysine27 at histone H3 (H3K27me2, H3K27me3) (Hsieh and Zhao, 2016).

Methylation can also be detected on the DNA itself. Here, methylation occurs at position 5 of cysteine residues (Yao and Jin, 2014). In most cases, these cysteine residues are immediately followed by a guanine residue ( $\mathrm{CpG}$ islands) (Simmen, 2008). The presence of methylated CpG islands is associated with gene silencing, for example the silencing of the $\mathrm{X}$ chromosome (Jaenisch and Bird, 2003) (Fig. 1.6C). Furthermore, during neurogenesis CpG methylation is involved in the silencing of pluripotency genes in neural progenitor cells (Mohn et al., 2008). Gene silencing via DNA methylation can occur by blockage of transcription factor binding, recruitment of methylcytosine binding proteins or induction of other repressive histone modifications (Cedar and Bergman, 2009).

DNA methylation can also occur on cysteine residues that are not adjacent to a guanine residue $(\mathrm{CpH})(\mathrm{Mo}$ et al., 2015). The formation of $\mathrm{CpH}$ islands, for example, could be detected in mature mouse cortical neurons (Mo et al., 2015). Interestingly, neuronal subtypes can be clearly differentiated by their specific methylation pattern, demonstrating the changes in DNA methylation pattern occurs during differentiation and specification (Hontelez et al., 2015; Mo et al., 2015). $\mathrm{CpH}$ methylation levels are low in actively transcribed genes and increase in silenced transcription factor positions on the DNA. So, by comparing the $\mathrm{CpH}$ methylation levels over time, the developmental history of cells in the adult embryo can be captured (Mo et al., 2015).

Interestingly, studies in $X$. tropicalis demonstrated that many epigenetic activating or repressing marks like $\mathrm{H} 3 \mathrm{~K} 4 \mathrm{me} 3$ and $\mathrm{H} 3 \mathrm{~K} 27 \mathrm{me} 3$ are maternally provided and are located close to the transcriptional start site of genes, while epigenetic marks achieved zygotically are located at a more distant position and are mainly close to enhancer elements (Hontelez et al., 2015).

The state of the chromatin itself can be changed by chromatin remodeling complexes such as the BAF chromatin remodeling complex (also known as the SWI/SNF complex), which is essential for vertebrate neurogenesis (Seo et al., 2005; Bachmann et al., 2016). The BAF complex consists of at least 15 subunits (Ho et al., 2009), with the subunits of the complex differing depending 
on the cell type or the time of expression (Lessard et al., 2007; Ho et al., 2009; Kadoch et al., 2013; Ronan et al., 2013; Narayanan and Tuoc, 2014). The BAF complex always contains either Brm or Brg1 as the catalytic subunit, and the three invariant core units BAF47, BAF155 and BAF170 (Lessard et al., 2007; Ronan et al., 2013). The remaining subunits show a high variability, where some subunits are more generally distributed, while many BAF complexes have their unique composition of subunits (Lessard et al., 2007; Ho et al., 2009; Kadoch et al., 2013; Ronan et al., 2013). Nevertheless, it has been demonstrated, that either Brg1 or Brm together with the three core units is sufficient to remodel nucleosomes (Phelan et al., 1999).

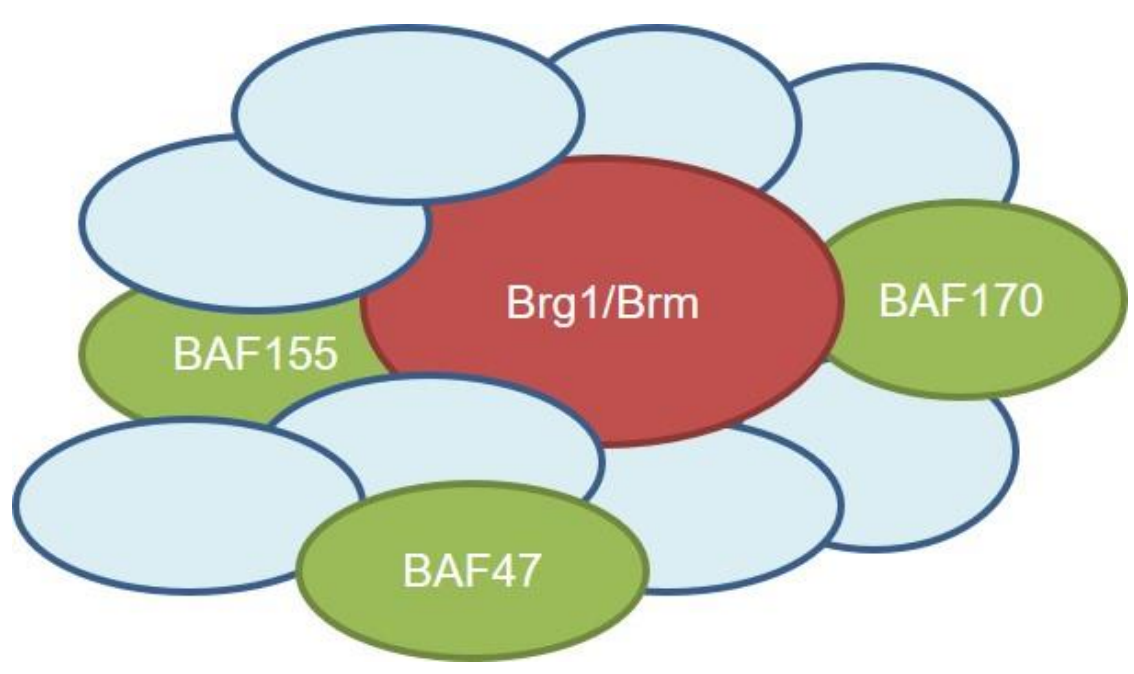

Figure 1.7 Composition of the BAF chromatin remodeling complex

The BAF chromatin remodeling complex consist of one of two catalytic core units (Brg1 or Brm) (red), three invariant core units (BAF47, BAF155 and BAF177) and a various number of context depending variant subunits (blue).

Neurogenesis is impaired in BAF mutants demonstrating the importance of chromatin remodeling via this complex (Matsumoto et al., 2006; Lessard et al., 2007; Narayanan and Tuoc, 2014). A loss of Brg1, for example, leads to severe defects in neurogenesis due to impairments in neuronal differentiation (Bachmann et al., 2016; Sokpor et al., 2017). Specific knock-out of Brg1 in murine neural progenitor cells leads to a reduced size of the brain, due to a lack of the cerebellum and a thinning of the midbrain, which results in a malformed cortex (Matsumoto et al., 2006; Lessard et al., 2007). Mice 
heterozygotic for Brg1 suffer from exencephaly, indicating a dosage dependency for Brg1 (Matsumoto et al., 2006; Lessard et al., 2007). Furthermore, a knock-down of Brg1 prevents the induction of neuronal differentiation by proneural genes such as neurog 2 or neurod 1 and leads to developmental arrest of $X$. laevis embryos at gastrula or open neural plate stage (Wagner et al., 2017).

Interestingly, the BAF chromatin remodeling complex can function as a transcriptional activator or as transcriptional repressor by directly binding to different proteins (Zhan et al., 2011; Tuoc et al., 2013). Basal expression of shh, for example, is repressed by BAF complex binding to Gli3, while interaction with Gli co-repressor histone deacetylase activates shh target genes (Zhan et al., 2011).

In addition to the BAF chromatin remodeling complex, there exist three other families of SWI-like ATPase dependent chromatin remodeling complex families, namely the ISWI complexes, the CHD complexes and the INO80 complex. ISWI complexes have SNF2H or SNF2L as their catalytic subunit and are involved in transcriptional activation and repression as well as the regulation of the chromatin structure, the replication of the DNA through heterochromatin and the segregation of chromosomes (Ho and Crabtree, 2010; Goodwin and Picketts, 2017). CHD complexes are subdivided in in three subfamilies. In mice, subfamily I members (CHD1 and CHD2) are mainly involved in the preservation of pluripotency (Gaspar-Maia et al., 2009), while subfamily II members (CHD3 and CHD4) are members of complexes containing histone deacetylases and function as transcriptional repressors (Zhang et al., 1998). CHD7, the best studied CHD family III member, however, is involved in transcriptional activation of tissue-specific genes during differentiation (Schnetz et al., 2009). Furthermore CHD8 homologue Duplin has been shown to be a negative regulator of canonical Wnt signaling in $X$. laevis (Heasman et al., 2000; Sakamoto et al., 2000). For nearly all of the CHD family members, essential regulatory functions during neurogenesis have been reported (Gaspar-Maia et al., 2009; Nieberler, 2012; Egan et al., 2013; Jones et al., 2015; Shen et al., 2015; Durak et al., 2016). A depletion CHD5 in the murine neocortex, for example, leads to an increase of undifferentiated progenitor cells and the expression of genes that normally are repressed 
(Egan et al., 2013), while CDH8 promotes the transcription of cell-cycle regulators (Durak et al., 2016). INO80 family members INO80 and SWR1 form large complexes with in vitro nucleosome-remodeling activity and are involved in transcriptional regulation (Bao and Shen, 2007).

\subsection{Changes in the epigenetic landscape during development}

At the time of fertilization, the fertilized oocyte is a totipotent cell, which can develop into cells of all three germ layers (Wobus and Boheler, 2005; Mitalipov and Wolf, 2009). During development, the cells become committed to a certain cell fate and so develop to multipotent progenitor cells, which then give rise to specialized cells (Wobus and Boheler, 2005; Mitalipov and Wolf, 2009). Since limitations in specification of the cells are achieved during development, the chromatin in the early $X$. tropicalis embryo or undifferentiated murine embryonic stem cells is characterized by quite accessible chromatin (Hontelez et al., 2015). In $X$ tropicalis embryos, the chromatin itself is relatively unmodified at early stages (Hontelez et al., 2015). While chromatin modifications increase during development, at the tailbud stage, $67 \%$ of the total chromatin still remains naïve (Hontelez et al., 2015), The first global changes in the chromatin landscape occurs at the transition from maternal to zygotic transcription, with an increase in histone marks such as H3K4me3 or $\mathrm{H} 3 \mathrm{~K} 9 \mathrm{ac}$ (Hontelez et al., 2015). Interestingly, most of H3K4me3 or H3K9ac marks are already established without the influence of zygotic factors and can be found even at promoters of late expressed genes, indicating that the presence of active histone marks is not sufficient to drive active transcription (Hontelez et al., 2015). This is supported by the observation that promoters of late expressed genes often are found in hypomethylated regions (Hontelez et al., 2015; Mo et al., 2015). However, after transcription the level of methylation at those genes increases, corresponding with gene silencing (Mo et al., 2015).

High levels of DNA methylation do not necessarily correspond to transcriptional repression, as relatively high DNA methylation upstream and downstream of promoter regions is still compatible with transcriptional activity (Bogdanovic et al., 2012). In addition, DNA methylation does not lead to 
transcriptional repression in early embryos, but does so in oocytes and late embryos (Bogdanovic et al., 2012). This is supported by the findings that epigenetic marks achieved by zygotic regulation in $X$. tropicalis were mostly located in regulatory regions of the DNA characterized by large distances from the promoter region and high degree of DNA methylation (Hontelez et al., 2015). Many marks for enhancers could be found in these regions, indicating that regulation via enhancers is not dependent on accessible chromatin (Hontelez et al., 2015).

Interestingly, several bHLH transcription factors involved in neuronal differentiation can work as pioneer transcription factors and do not need accessible chromatin to induce target gene expression. The neuronal differentiation gene Neurod1, for example, can induce target gene activation prior to chromatin opening (Pataskar et al., 2016). Furthermore, Neurod1 itself can alter the state of the chromatin surrounding its target genes (Pataskar et al., 2016). Other bHLH transcription factors like Neurog2 and Ascl1 have also been shown to bind non-accessible regions on the DNA (Smith et al., 2016).

\subsection{Ptf1a}

Pancreatic transcription factor 1a (Ptf1a) is a bHLH transcription factor that was first identified as a member of a trimeric transcription factor complex involved in the development of the exocrine pancreas (Cockell et al., 1989). In $X$. laevis, Ptf1a expression can first be detected in two longitudinal stripes along the dorsal midline. During subsequent stages of development, ptf1a expression becomes restricted to the developing hindbrain, spinal cord, the retina and the early pancreas anlagen (Afelik et al., 2006) (Fig. 1.7A). Transversal sections at tailbud stage indicate that the expression domain of Ptf1a in the neural tube is restricted to the intermediate zone, in which the dorsal inhibitory interneuron population arise (Fig 1.7B).

Ptf1a is best characterized for its role during development of the pancreas, where it is involved in the specification of pancreatic precursor cells (Kawaguchi et al., 2002; Afelik et al., 2006). At later stages, it contributes to 
the formation of the exocrine pancreas and the induction of digestive enzymes (Cockell et al., 1989; Krapp et al., 1996; Krapp et al., 1998; Kawaguchi et al., 2002). The importance of Ptf1a in pancreas development can be demonstrated in ptf1a knockout-mice, which suffer from a complete loss of the exocrine pancreas and while just a few endocrine cells are present, they are mislocalized to the spleen (Krapp et al., 1998). Correspondingly, a knock-down of ptf1a in $X$. laevis and $X$. tropicalis embryos leads to the loss of exocrine marker genes and pancreatic tissue (Afelik et al., 2006; Lei et al., 2012). Furthermore, mutations affecting Ptf1a activity are found in humans who suffer from neonatal diabetes due to pancreatic agenesis (Hoveyda et al., 1999; Sellick et al., 2004; Tutak et al., 2009; Al-Shammari et al., 2011; Weedon et al., 2014).
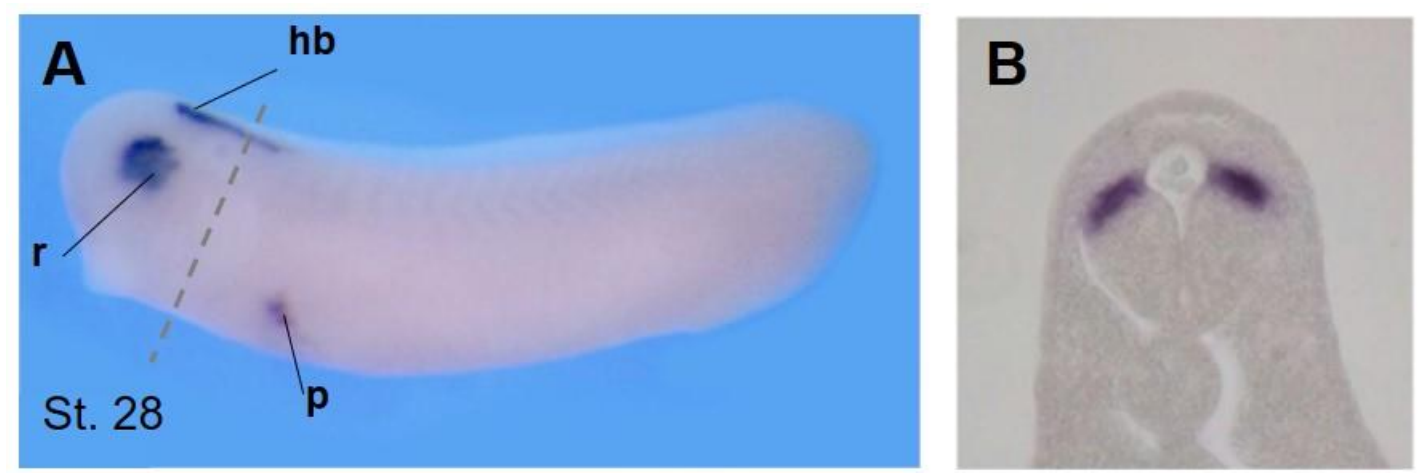

\section{Figure 1.8: Expression pattern of Ptf1a}

(A) At tailbud stage $X$. laevis embryos, Ptf1a is expressed in the retina (r), the hindbrain (hb) and the early pancreas anlagen ( $p)$. Dashed line indicating the positioning of the cross-section on the right. $r$ : retina, $h b$ : hindbrain, $p$ : pancreas (B) Transversal section of $X$. laevis at the level of the hindbrain. Ptf1a is expressed in the dorsal in the progenitor domain, where the dorsal interneuron populations arise.

In addition to its essential role in pancreas development, Ptf1a is also required during early embryogenesis for the establishment of the nervous system (Sellick et al., 2004; Glasgow et al., 2005; Hoshino et al., 2005; Dullin et al., 2007; Nakhai et al., 2007; Pascual et al., 2007; Hanotel et al., 2014). In this context, Ptf1a is best characterized for its ability to drive the development of GABAergic inhibitory neurons at the expanse of a glutamatergic cell fate in the retina, the hindbrain and the dorsal spinal cord (Glasgow et al., 2005; Hoshino et al., 2005; Fujitani et al., 2006; Dullin et al., 2007; Pascual et al., 2007). In 
the mouse hindbrain and dorsal spinal cord, Ptf1a controls the development of dl4 and dlLA interneuron populations that express the homeodomain transcription factors pax2 and Ihx1/5 (Glasgow et al., 2005). In Ptf1a null mutant mice, those progenitor domains are completely absent, while the dl5 progenitor population is increased (Glasgow et al., 2005). In the cerebellum, Ptf1a is essential for the formation of all types of GABAergic neurons (Hoshino et al., 2005; Kani et al., 2010; Mizuhara et al., 2010; Nishida et al., 2010), while in the retina, it drives the development of GABAergic amacrine and horizontal cells at the expanse of retinal ganglion and photoreceptor cells (Fujitani et al., 2006; Dullin et al., 2007; Nakhai et al., 2007; Lelievre et al., 2011). However, lineage tracing of Ptf1a positive cells in mice and chick embryos demonstrate that progenitors expressing Ptf1a can also develop into other neuronal subtypes. For example, Ptf1a cooperates with Olig3 in the differentiation of glutamatergic climbing fiber neurons in the inferior olivary nucleus (Yamada et al., 2007; Storm et al., 2009), while subpopulations of Ptf1a expressing cells in the dorsal spinal cord will give rise to glycinergic neurons (Huang et al., 2008; Bessodes et al., 2017). Furthermore, it has been suggested that Ptf1a expressing cells might also be involved in the formation of serotonergic neurons in the zebrafish enteric nervous system (Uribe et al., 2016).

Besides its involvement in driving the development to distinct neuronal subtypes, Ptf1a also promotes neuronal differentiation, with overexpression of Ptf1a in $X$. laevis embryos and ectodermal explants being sufficient to ectopically drive the non-neural ectodermal cells to develop into post-mitotic neurons (Dullin et al., 2007). Interestingly, although Ptf1a and Neurog2 both promote general neuronal differentiation, Neurog2 drives the development of glutamate expressing excitatory neurons (Patterson and Krieg, 1999; Perron et al., 1999), while Ptf1a promotes a GABAergic cell fate (Glasgow et al., 2005; Hoshino et al., 2005; Li et al., 2006; Dullin et al., 2007; Hedderich, 2008). Overexpression of Neurog2 and Ptf1a in $X$. laevis embryos and animal caps demonstrates that Ptf1a can alter the neuronal transmitter phenotype induced by Neurog2 to a GABAergic cell fate (Hedderich, 2012). In a similar way, misexpression of Ptf1a in ascl1 expressing cells will also change the induced neuronal transmitter cell fate from glutamatergic to GABAergic (Chang et al., 2013; Borromeo et al., 2014). 


\subsection{Ptf1a forms a trimeric transcription factor complex}

Ptf1a is a bHLH protein that functions as a heterotrimeric transcription complex that recognizes a bipartite binding motif on target genes (Roux et al., 1989; Krapp et al., 1996; Beres et al., 2006; Masui et al., 2007; Hori et al., 2008). Ptf1a binds with a ubiquitously expressed class A bHLH transcription factor, (E2A/p75/HEB/TCF12), which recognizes E-box motifs (CANNTG) on the DNA (Murre et al., 1989; Beres et al., 2006). Furthermore, Ptf1a binds to a CSL family member, which binds to an adjacent TC box motif (TTTCCCA) on the DNA, in a distance of 1-3 helical turns (Beres et al., 2006; Meredith et al., 2013) (Fig. 1.8A). In mammals, two closely related CSL family members are found, Rbpl and Rbpj. While Rbpj is the component of the Ptf1a trimeric complex in the nervous system and pancreatic progenitor cells, the interaction with Rbpl is restricted to the adult pancreatic tissue (Beres et al., 2006; Hori et al., 2008). In contrast to mammals, there are two homeologs of the CSL family in X. laevis and both are closely related to Rbpj (Beres et al., 2006). The interaction with mouse $\mathrm{Rbpj}$ is mediated by two tryptophan residues within two highly conserved motifs on the Ptf1a C-terminus (C1: HSLSW and C2: VWTPEDPR) (Beres et al., 2006; Hori et al., 2008). This interaction can be disrupted through mutation of the tryptophan residues in the $\mathrm{C} 1$ and $\mathrm{C} 2$ domain to alanine (Hori et al., 2008). While the C1 domain of the mouse Ptf1a is more important for binding Rbpl, mutation of the $\mathrm{C} 2$ domain is sufficient for disruption of binding of Ptf1a to Rbpj (Beres et al., 2006). Complete disruption of Ptf1a with $X$. laevis $\mathrm{Rbpj}$ requires mutation at the corresponding tryptophan residues in both the C1 and C2 domains (Ptf1a 2 224A/W242A $)$ (Hanotel et al., 2014).

The binding of Rbpj is thought to be essential for Ptf1a function, since mutations that results in truncation at the Ptf1a C-terminus have been found in children suffering from cerebellar agenesis (Sellick et al., 2004). Furthermore, mutations in the Ptf1a C2 domain in chick embryos result in loss of GABAergic dl4 and dILA neurons, a phenotype that is similar to those of Ptf1a knock-out mice (Hori et al., 2008). Interestingly, overexpression of a Ptf1a C1C2 mutant $\left(P t f 1 a^{\mathrm{W} 224 \mathrm{~A} / 242 \mathrm{~A}}\right)$ in $X$. laevis embryos and $\mathrm{C}$-terminal truncation mutants known to cause cerebellar agenesis in humans, still induce neuronal differentiation (Hedderich, 2008; Hedderich, 2012; Richts, 2013). However, the induced 
neuronal subtype is changed from a GABAergic to glutamatergic cell fate (Fig 1.8B). Loss of Ptf1a in the mouse brain will not affect the generation of the formerly Ptf1a expressing progenitor populations, but instead, those cells will either migrate to the brainstem or undergo apoptosis (Millen et al., 2014; Iskusnykh et al., 2016).

Rbpj not only binds to Ptf1a, but also binds to the Notch intracellular domain (Nishimura et al., 1998). It has been shown that the interaction of Ptf1a and NICD with Rbpj is mutually exclusive, suggesting that Ptf1a and NICD might compete with each other in binding Rbpj (Beres et al., 2006).

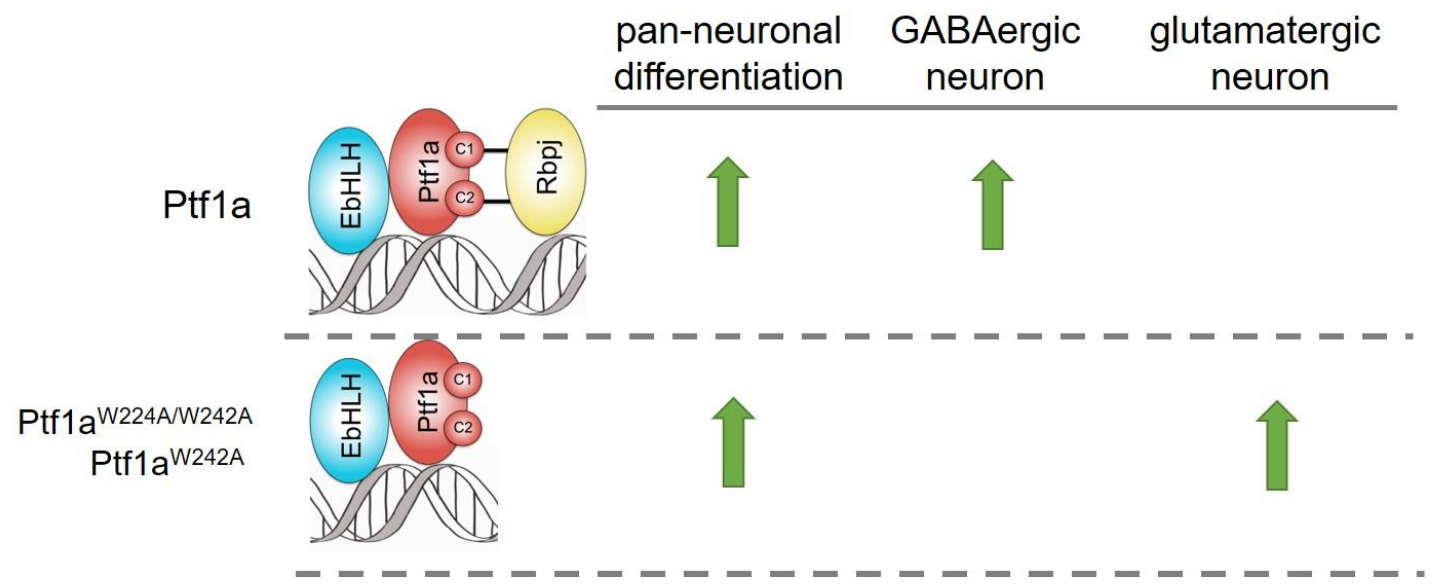

Figure 1.9: Ptf1a forms a heterotrimeric complex

Ptf1a (green) forms a trimeric complex with a class $\mathrm{A}$ bHLH protein (blue), binding an E-box motif on the DNA and to Rbpj (orange) binding an adjacent TC-box motif. Interaction with Rbpj is established by two highly conserved motifs ( $\mathrm{C} 1$ and $\mathrm{C} 2$ ). Interaction with Rbpj can be disrupted by inducing point mutations from tryptophan to alanine within these motifs. While both mutants can promote neuronal differentiation, the wild-type Ptf1a promotes a GABAergic cell fate, while the Ptf1 $\mathrm{a}^{\mathrm{W} 224 \mathrm{~A}}$ and Ptf1a ${ }^{\text {W224A/242A }}$ mutant induces a glutamatergic cell fate.

\subsection{Gene induction and regulation by Ptf1a}

In the developing pancreas, Ptf1a directly binds the $p d x 1$ promoter and drives the development of the early pancreas (Wiebe et al., 2007). Furthermore, Ptf1a is well known for activating genes associated with the maturation of the exocrine pancreas and for the induction of digestive enzymes (Cockell et al., 1989; Krapp et al., 1996; Krapp et al., 1998; Kawaguchi et al., 2002; Masui et al., 2010). Ptf1a is autoregulated by binding to its own promoter in a positive feedback loop (Masui et al., 2008). 
In contrast to pancreas development, only a few downstream target genes of Ptf1a are known during neurogenesis. Ptf1a directly activates neurog2 expression in chick and mouse embryos (Henke et al., 2009). Furthermore, the two immunoglobulin superfamily members, nephrin and neph3 (also known as kirrel2) are direct target genes of Ptf1a in mouse (Nishida et al., 2010). As kirrel2 is expressed in Purkinje progenitor cells, it is suggested that its activation by Ptf1a plays a role in GABAergic neuronal subtype specification (Mizuhara et al., 2010). As in pancreas development, it has been shown that Ptf1a expression is under the control of a positive feedback loop, by binding to its own promoter (Meredith et al., 2009). Furthermore, barh/2 could be shown to act downstream of Ptf1a in the development of amacrine cells in the zebrafish retina (Jusuf et al., 2012). In addition, the zinc finger histone methyltransferase gene Prdm13 has been shown to be a direct Ptf1a target gene in mouse and $X$. laevis and is required to repress a glutamatergic excitatory neuronal cell fate (Chang et al., 2013; Hanotel et al., 2014). Prdm13 inhibits the Neurog2- or Ascl1-induced expression of the glutamatergic selector gene $t / x 3$ via interaction with its regulatory sequences and direct interaction with Ascl1 (Chang et al., 2013; Hanotel et al., 2014; Mona et al., 2017). In addition, Prdm13 inhibits the expression of its own inducer, Ptf1a, in subpopulations of Ptf1a expressing cells that will develop into glycinergic cell fate (Bessodes et al., 2017; Mona et al., 2017). In X. laevis ectodermal explants, activation of direct target genes by Ptf1a underlies a temporal selection (Hedderich, 2012). While some direct target genes like prdm13 are activated immediately by Ptf1a, other direct target genes like neurog2 undergo a delayed activation (Hedderich, 2012).

Similar to many bHLH proteins, Ptf1a contains S/T-P residues, which are putative targets of Cdk-mediated phosphorylation (Ubersax and Ferrell, 2006). Preventing phosphorylation at those sites, through mutation of these sites in Neurog2, leads to an increase in activation of the pan-neuronal marker tubb2b (Ali et al., 2011). No increase in neuronal inducing activity of Ptf1a was detected upon overexpression of a murine Ptf1a in which all eight S/T-P residues were mutated to alanine $\left(\mathrm{mPtf} 1 \mathrm{a}^{8 \mathrm{~S} / \mathrm{T} \rightarrow \mathrm{A}}\right)$ in $X$. laevis embryos and animal caps, (Richts, 2013). Surprisingly, additional to its ability to induce markers for a GABAergic cell fate, mPtf1 $\mathrm{a}^{\mathrm{BS} / \mathrm{T} \rightarrow \mathrm{A}}$ also induced the glutamatergic 
marker gene $t / x 3$, a gene that is normally repressed by Ptf1a. Mutating the alanine residue at position 299 back to threonine $\left(\mathrm{mPtf} \mathrm{a}^{7 \mathrm{~S} \rightarrow \mathrm{A}}\right)$ could prevent the mixed induced neuronal transmitter phenotype back to an induced GABAergic cell fate (Richts, 2013), suggesting that the mutation of the $T_{299}$ was responsible for this activity.

\subsection{Aims}

The bHLH transcription factor Ptf1a is sufficient to promote neuronal differentiation (Dullin et al., 2007; Hedderich, 2008; Hedderich, 2012) and is able to drive the development of a GABAergic neuronal subtype at the expense of a glutamatergic neuronal subtype in the vertebrate brain, dorsal spinal cord, and retina (Glasgow et al., 2005; Hoshino et al., 2005; Dullin et al., 2007; Pascual et al., 2007). In recent years, Ptf1a mutants were identified that alter the induced neuronal transmitter phenotype (Hedderich, 2008; Hori et al., 2008; Richts, 2013). In this thesis, the selective induction of target genes by Ptf1a will be further analyzed. Therefore, Ptf1a $\mathrm{C} 2$ domain mutants inducing various neuronal call fates will be investigated by their activation of target genes and for potential mechanisms causing the alterations in the induced neuronal subtypes by those mutants. Furthermore, the function of chromatin remodeling in controlling the delay in the activation of target genes by Ptf1a will be analyzed. This will be investigated by studying the dependency of Ptf1a target gene activation on chromatin remodeling by the BAF complex and directly studying changes chromatin accessibility over time. 


\section{Materials and Methods}

\subsection{Materials}

\subsubsection{X. laevis}

$X$. laevis pigmented males and females were purchased from Nasco (Ft. Atkinson, USA). Developmental stages were defined according to Nieuwkoop and Faber (1967).

\subsubsection{Bacteria}

The chemical competent Escherichia coli strand XL1-Blue (recA1, endA1, gyrA96, thi-1, hsdR17, supE44, relA1, lac[FproAB, laclqZ $\Delta M 15, \operatorname{Tn} 10\left(\right.$ Tet $\left.\left.^{r}\right)\right]^{c}$ ) was used for molecular biology standard methods.

\subsubsection{Chemicals}

Chemicals used were ordered from the following companies: ApplChem, Merck, Roche, Roth, Sigma-Aldrich

\subsubsection{Antibiotics and Media}

Bacteria culture medium was made by dissolving $32 \mathrm{~g}$ Luria Bertani (LB) in $1 \mathrm{I}$ $\mathrm{dH}_{2} \mathrm{O}$. LB Medium was autoclaved at $121^{\circ} \mathrm{C}$ and then cooled down on $50{ }^{\circ} \mathrm{C}$. Ampicillin (working concentration: $100 \mu \mathrm{g} / \mathrm{ml}$, diluted from a $100 \mathrm{mg} / \mathrm{ml}$ in $\mathrm{dH}_{2} \mathrm{O}$ stock solution) was added and the medium transferred to petri dishes and stored at $4{ }^{\circ} \mathrm{C}$. 


\subsubsection{Oligonucleotides}

Oligonucleotides for cloning and RT-PCR were purchased from Sigma-Aldrich and were dissolved in $\mathrm{dH}_{2} \mathrm{O}$ to a $100 \mu \mathrm{M}$ or $500 \mu \mathrm{M}$ stock solution. Antisense morpholino oligonucleotides were purchased from Gene Tools and dissolved in RNase-free water to a concentration of $20 \mathrm{ng} / \mathrm{nl}$. The morpholinos were stored at $4{ }^{\circ} \mathrm{C}$ and were heated for $5 \mathrm{~min}$ at $65^{\circ} \mathrm{C}$ before use.

\subsubsection{RT-PCR oligonucleotides}

As $X$. laevis is allotetraploid all chromosomes are present in a large (.L) and a small (.S) copy. The homeolog targeted by the oligonucleotide is indicated behind the gene name.

\begin{tabular}{|c|c|c|c|c|}
\hline Oligonucleotide & $\begin{array}{l}\text { Orien- } \\
\text { tation }\end{array}$ & Sequence & $\mathrm{T}_{\mathrm{A}}{ }^{\circ} \mathrm{C}$ & Cycles \\
\hline $\begin{array}{l}\text { odc.L } \\
\text { (Heasman et al., } \\
2000 \text { ) }\end{array}$ & for & $\begin{array}{l}5^{\prime}- \\
\text { GCCATTGTGAAGACTCTCT } \\
\text { CCATTC-3' }\end{array}$ & 57 & 25 \\
\hline $\begin{array}{l}\text { odc.L } \\
\text { (Heasman et al., } \\
2000 \text { ) }\end{array}$ & rev & $\begin{array}{l}\text { 5'- } \\
\text { TTCGGGTGATTCCTTGCCA } \\
\text { C-3' }\end{array}$ & 57 & 25 \\
\hline $\begin{array}{l}\text { n-tubulin. } L+S \\
\text { (Stancheva and } \\
\text { Meehan, 2000) }\end{array}$ & for & $\begin{array}{l}5^{\prime}- \\
\text { ACACGGCATTGATCCTACA } \\
\text { G-3' }\end{array}$ & 56 & 32 \\
\hline $\begin{array}{l}n \text {-tubulin. } L+S \\
\text { (Stancheva and } \\
\text { Meehan, 2000) }\end{array}$ & rev & $\begin{array}{l}5^{\prime}- \\
\text { AGCTCCTTCGGTGTAATGA } \\
\text { C-3' }\end{array}$ & 56 & 32 \\
\hline $\begin{array}{l}\text { gad1a.L } \\
\text { (Dullin et al., 2007) }\end{array}$ & for & $\begin{array}{l}5^{\prime} \text { - } \\
\text { ATGGGCGTCTTACTCCAAT } \\
\text { G-3' }\end{array}$ & 60 & 32 \\
\hline $\begin{array}{l}\text { gad1a.L } \\
\text { (Dullin et al., 2007) }\end{array}$ & rev & $\begin{array}{l}5^{\prime}- \\
\text { ATGTCTACATGGCGACCA } \\
\text { CA-3' }\end{array}$ & 60 & 32 \\
\hline
\end{tabular}




\begin{tabular}{|l|l|l|l|l|}
\hline $\begin{array}{l}\text { tlx3.S } \\
\text { (Dullin et al., 2007) }\end{array}$ & for & $\begin{array}{l}5^{\prime} \text { - } \\
\text { GCCAACAAGTACAAGTGC } \\
\text { ACAG-3' }\end{array}$ & 62 & 27 \\
\hline $\begin{array}{l}\text { tlx3.S } \\
\text { (Dullin et al., 2007) }\end{array}$ & rev & $\begin{array}{l}5^{\prime}- \\
\text { CAGGAGCCAGACTCACAT } \\
\text { TGAC-3' }\end{array}$ & 62 & 27 \\
\hline $\begin{array}{l}\text { prdm13.S } \\
\text { (Hanotel et al., } \\
\text { 2014) }\end{array}$ & for & $\begin{array}{l}5^{\prime} \text { - } \\
\text { CTGCCGACACATGATGAA } \\
\text { AAAGG-3' }\end{array}$ & 63 & 27 \\
\hline $\begin{array}{l}\text { prdm13.S } \\
\text { (Hanotel et al., } \\
\text { 2014) }\end{array}$ & rev & $\begin{array}{l}5^{\prime}- \\
\text { AGATTTTGGGGGAGGCAG } \\
\text { AAAAG-3' }\end{array}$ & 63 & 27 \\
\hline neurog2.L & for & $\begin{array}{l}5^{\prime}- \\
\text { TTTGTTAAGGGCGAATGTC } \\
\text { A-3' }\end{array}$ & $\begin{array}{l}5^{\prime} \text { - } \\
\text { ATCCGGGAAGGTGAAAA } \\
\text { GT-3' }\end{array}$ & 34 \\
\hline neurog2.L & rev & 62 & 34 \\
\hline
\end{tabular}

Table 2.1 List of RT-primers and their PCR parameters

\subsubsection{Sequencing oligonucleotides}

\begin{tabular}{|l|l|}
\hline Oligonucleotide & Sequence \\
\hline T3 & 5'-TTAACCCTCACTAAAGG-3' \\
\hline T7 & 5'-TAATACGACTCACTATAGG-3' \\
\hline SP6 & 5'-ATTTAGGTGACACTATAG-3' \\
\hline Ptf1a rev & 5'-CATCAGTCCATGAGAGAG-3' \\
\hline
\end{tabular}

Table 2.2 List of sequencing oligonucleotides 


\subsubsection{Morpholino oligonucleotides}

\begin{tabular}{|l|l|}
\hline Morpholino & Sequence \\
\hline Brg1-MO (Seo et al., 2005) & 5'-TCACTGCTAACCTGTCCCCGAATCC-3' \\
\hline $\begin{array}{l}\text { Standard control MO } \\
\text { (GeneTools) }\end{array}$ & 5'-CCTCTTACCTCAGTTACAATTTATA-3' \\
\hline
\end{tabular}

Table 2.3 List of morpholino oligonucleotides

\subsubsection{Sense RNA constructs}

\begin{tabular}{|c|c|c|c|c|}
\hline Construct & Vector & $\begin{array}{c}\text { Restriction } \\
\text { enzyme }\end{array}$ & Polymerase & Reference \\
\hline Ptf1a & $\mathrm{pCS} 2+\mathrm{GR}$ & Notl & SP6 & $\begin{array}{c}\text { (Afelik et al., } \\
2006)\end{array}$ \\
\hline Ptf1aW242A & $\mathrm{pCS} 2+\mathrm{GR}$ & Notl & SP6 & $\begin{array}{c}\text { (Hanotel et al., } \\
\text { 2014) }\end{array}$ \\
\hline Ptf1aW224A/W242A & $\mathrm{pCS} 2+\mathrm{GR}$ & Notl & SP6 & $\begin{array}{c}\text { (Hanotel et al., } \\
\text { 2014) }\end{array}$ \\
\hline$P t f 1 a^{T 243 A}$ & pCS2+GR & Notl & SP6 & This thesis \\
\hline$P t f 1 a^{T 243 E}$ & $\mathrm{pCS} 2+\mathrm{GR}$ & Notl & SP6 & This thesis \\
\hline Ptf1a-Vn & $\mathrm{pCS} 2+\mathrm{Vn}$ & Notl & SP6 & $\begin{array}{c}\text { (Hedderich, } \\
\text { unpublished } \\
\text { data) }\end{array}$ \\
\hline $\begin{array}{l}\text { Ptf1 } 1 a^{\text {W224AW242A }} \\
\text { Vn }\end{array}$ & $\mathrm{pCS} 2+\mathrm{Vn}$ & Notl & SP6 & $\begin{array}{c}\text { (Hedderich, } \\
\text { unpublished } \\
\text { data) }\end{array}$ \\
\hline Ptf1a $1243 \mathrm{~A}-\mathrm{Vn}$ & $\mathrm{pCS} 2+\mathrm{Vn}$ & Notl & SP6 & This thesis \\
\hline Ptf1a $1243 E-V n$ & $\mathrm{pCS} 2+\mathrm{Vn}$ & Notl & SP6 & This thesis \\
\hline Rbpj-Vc & $\mathrm{pCS} 2+\mathrm{Vc}$ & Notl & SP6 & $\begin{array}{c}\text { (Hedderich, } \\
\text { unpublished } \\
\text { data) }\end{array}$ \\
\hline Prdm13-Vc & $\mathrm{pCS} 2+\mathrm{Vc}$ & Notl & SP6 & This thesis \\
\hline Neuroq2-Vn & $\mathrm{pCS} 2+\mathrm{Vn}$ & Notl & SP6 & This thesis \\
\hline
\end{tabular}




\begin{tabular}{|c|c|c|c|c|}
\hline E12-Vc & pCS2+Vc & Notl & SP6 & $\begin{array}{c}\text { (Hedderich, } \\
\text { unpublished } \\
\text { data) }\end{array}$ \\
\hline B-Gal & pCS2+ & Notl & SP6 & $\begin{array}{c}\text { (Chitnis and } \\
\text { Kintner, 1995) }\end{array}$ \\
\hline mRFP & pCS2+ & Notl & SP6 & $\begin{array}{c}\text { (Saka et al., } \\
\text { 2007) }\end{array}$ \\
\hline
\end{tabular}

Table 2.4 List of overexpression constructs

\section{Overexpression constructs:}

E12-VcpCS2+ (prepared by M. Hedderich and K. Ditter):

$\mathrm{E} 12-\mathrm{V} c p C S 2+$ contains the open reading frame of Rbpj fused at its $\mathrm{N}$-terminus with the C-terminus of Venus YFP (aa 155-238). E12-VcpCS2+, was generated by PCR amplification of the E12 open reading frame using XE12pSP64XBm (obtained from Eric Bellefroid) as a template and the following primers: for, 5'-GTACCGGTGGTGGTGGTGGTGGTAATCAGCAG CAGAGGATG-3' and rev, 5'-GGGTACGTATCACATGTGTCCCACTGG-3'. The PCR product was inserted into VcpCS2+ (Saka et al., 2007) using Agel and SnaBI restriction sites. For preparation of sense RNA, the construct was linearized with Notl and transcribed with SP6 polymerase.

Ptf1a-VnpCS2+ (prepared by M. Hedderich and K. Ditter):

Ptf1a-VnpCS2+ contains the open reading frame of Ptf1a fused at its $\mathrm{N}$ terminus with the N-terminus of Venus YFP (aa 1-154). Ptf1a-VnpCS2+, was generated by PCR amplification of the Ptf1a open reading frame using Ptf1aGRpCS2+ (Afelik et al., 2006) as a template and the following primers: for, 5'TACCGGTGGTGGTGGTGGTGGTGAAACGGTCCTGGAGC-3' and rev, 5'GGGTACGTATCACATATCAAGGCACAAAG-3'. The PCR product was inserted into VnpCS2+ (Saka et al., 2007) using Agel and SnaBI restriction sites. For preparation of sense RNA, the construct was linearized with Notl and transcribed with SP6 polymerase. 
Rbpj-VcpCS2+ (prepared by M. Hedderich and K. Ditter):

Rbpj-VcpCS2+ contains the open reading frame of Rbpj fused at its $\mathrm{N}$-terminus with the C-terminus of Venus YFP (aa 155-238). Rbpj-VcpCS2+, was generated by PCR amplification of the Rbpj open reading frame using RbpjGRpCS2+ (Hedderich, 2012) as a template and the following primers: for, 5'GTACCGGTGGTGGTGGTGG TGGTCAACCTGGCATTCCTAAATAC-3' and rev, 5'-GGGTACGTATTAGGACACTACTGCTGCAGTG-3'. The PCR product was inserted into $\mathrm{VcpCS2}+$ (Saka et al., 2007) using Agel and SnaBI restriction sites. For preparation of sense RNA, the construct was linearized with Notl and transcribed with SP6 polymerase.

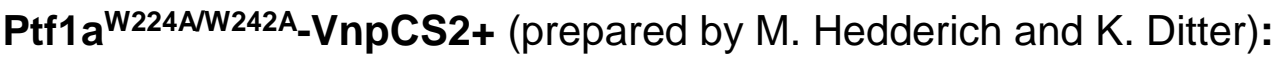

Ptf1a ${ }^{\text {W224AW242A }-V n p C S 2}+$ contains the open reading frame of Ptf1a fused at its $\mathrm{N}$-terminus with the $\mathrm{N}$-terminus of Venus YFP (aa 1-154). Ptf1aW224AW242A VnpCS2+, was generated by PCR amplification of Ptf1a open reading frame using Ptf1aW224AW242A-GRpCS2+ (Hedderich, 2008) as a template and the following primers: for, 5'-TACCGGTGGTGGTGGTGGTGGTGAAACGGT CCTGGAGC-3' and rev, 5'-GGGTACGTATCACATATCAAGGCACAAAG-3'. The PCR product was inserted into VnpCS2+ (Saka et al., 2007) using Agel and $\mathrm{SnaBI}$ restriction sites. For preparation of sense RNA, the construct was linearized with Notl and transcribed with SP6 polymerase.

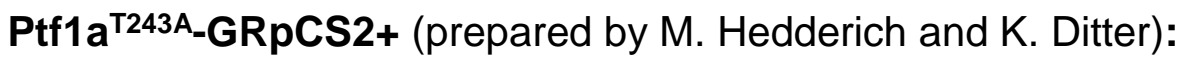

Ptf1a ${ }^{\text {T243A-GRpCS2 }}$ + contains the open reading frame of Ptf1a with putative phosphorylation site threonine 243 mutated to alanine. To synthesize

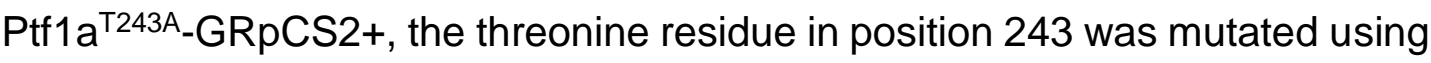
the QuickChange XL Site Directed Mutagenesis Kit (Stratagene) and the following primers (mutations are indicated in red): for, 5'-GGCCAAAGTGTGG GCTCCTGAGGATCCC-3' and rev, 5'-GGGATCCTCAGGAGCCCACAC TTTGGCC-3' and Ptf1a-GRpCS2+ as a template. For preparation of sense RNA, the construct was linearized with Notl and transcribed with SP6 polymerase. 


\section{Ptf1a ${ }^{\text {T243E-GRpCS2+: }}$}

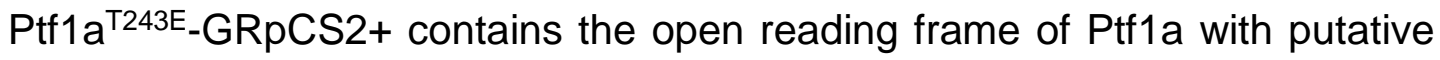
phosphorylation site threonine 243 mutated to glutamate. To synthesize

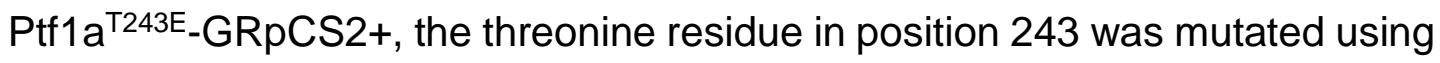
the QuickChange XL Site Directed Mutagenesis Kit (Stratagene) and the following primers (mutations are indicated in red): for, 5'-CGGC CAAAGTGTGGGAGCCTGAGGATCCCAGG-3' and rev, 5'-CCTGGGATCC TCAGGCTCCCACACTTTGGCCG-3' and Ptf1a-GRpCS2+ as a template. For preparation of sense RNA, the construct was linearized with Notl and transcribed with SP6 polymerase.

\section{Ptf1a ${ }^{\text {T243A-VnpCS2+: }}$}

Ptf1aT243A-VnpCS2+ contains the open reading frame of Ptf1 $\mathrm{a}^{\mathrm{T} 243 \mathrm{~A}}$ fused at its $\mathrm{N}$-terminus with the $\mathrm{N}$-terminus of Venus YFP (aa 1-154). To synthesize

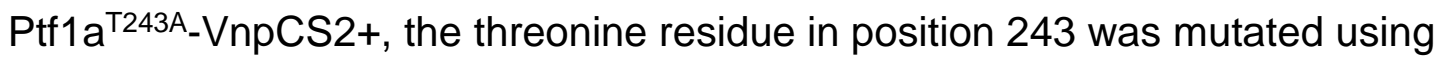
the QuickChange XL Site Directed Mutagenesis Kit (Stratagene) and the following primers (mutations are indicated in red): for, 5'-GGCCAAAGT GTGGGCTCCTGAGGATCCC-3' and rev, 5'-GGGATCCTCAGGAGCCC ACACTTTGGCC-3' and Ptf1a-VnpCS2+ as a template. For preparation of sense RNA, the construct was linearized with Notl and transcribed with SP6 polymerase.

\section{Ptf1a ${ }^{\text {T243E }}$-VnpCS2+:}

Ptf1 $a^{T 243 E}-V n p C S 2+$ contains the open reading frame of Ptf1a ${ }^{T 243 E}$ fused at its $\mathrm{N}$-terminus with the $\mathrm{N}$-terminus of Venus YFP (aa 1-154). To synthesize

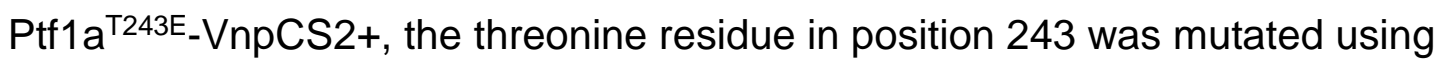
the QuickChange XL Site Directed Mutagenesis Kit (Stratagene) and the following primers (mutations are indicated in red): for, 5'-CCTGGGATC CTCAGGCTCCCACACTTTGGCCG-3' and rev, 5'-CGGCCAAAGTGTG GGAGCCTGAGGATCCCAGG-3' and Ptf1a-VnpCS2+ as a template. For preparation of sense RNA, the construct was linearized with Notl and transcribed with SP6 polymerase. 


\section{Neurog2-VnpCS2+:}

Neurog2-VnpCS2+ contains the open reading frame of Neurog2 fused at its $\mathrm{N}$-terminus with the N-terminus of Venus YFP (aa 1-154). Neurog2-VnpCS2+, was generated by PCR amplification of the Neurog2 open reading frame using Neurog2-HApCS2+ (Hedderich, unpublished data) as a template and the following primers: for, 5'-GTACCGGTGGTGGTGGTGGTGGTGTGCT GCTCAAGTGCG-3' and rev, 5'-GGGTACGTATCAAATGAAAGCGCTGCT$3^{\prime}$. The PCR product was inserted into VnpCS2+ (Saka et al., 2007) using the Agel and SnaBI restriction sites. For preparation of sense RNA, the construct was linearized with Notl and transcribed with SP6 polymerase.

\section{Prdm13-VcpCS2+:}

Prdm13-VcpCS2+ contains the open reading frame of Prdm13 fused at its $\mathrm{N}$ terminus with the C-terminus of Venus YFP (aa 155-238). Prdm13-VcpCS2+, was generated by PCR amplification of the Prdm13 open reading frame using cDNA as a template and the following primers: for, 5'ATGCATTGCAACAGGGCTC-3' and rev, 5'-TTAGGGTTCCTTGCTG CTTCC-3'. The insert was subcloned into pGemTeasy vector, which was then used as a template for PCR amplification of the Prdm13 open reading frame using the following primers: for, 5'-GTACCGGTGGTGGTGGTGGTGGTC ATTGCAACAGGGCTCTG-3' and rev, 5'-GGGTACGTATTAGGGTTCC TTGCTGCTTC-3'. The PCR product was inserted into VcpCS2+ (Saka et al., 2007) using the Agel and SnaBI restriction sites. For preparation of sense RNA, the construct was linearized with Notl and transcribed with SP6 polymerase.

\subsubsection{Antisense RNA constructs}

\begin{tabular}{|l|c|c|c|l|}
\hline Construct & Vector & $\begin{array}{c}\text { Restriction } \\
\text { enzyme }\end{array}$ & Polymerase & \multicolumn{1}{|c|}{ Reference } \\
\hline tubb2b & pBst KS & BamHI & T3 & $\begin{array}{l}\text { (Chitnis and } \\
\text { Kintner, 1995) }\end{array}$ \\
\hline gad1a & $\begin{array}{c}\text { pCMV- } \\
\text { Sport6ccdB }\end{array}$ & Notl & SP6 & (Dullin et al., 2007) \\
\hline
\end{tabular}




\begin{tabular}{|l|l|l|l|l|}
\hline tlx3 & BStEll & Stu1 & T3 & $\begin{array}{l}\text { (Patterson and } \\
\text { Krieg, 1999) }\end{array}$ \\
\hline
\end{tabular}

Table 2.5 List of RT-primers and their PCR parameters

\subsection{Methods}

\subsubsection{In vitro synthesis of capped sense RNA}

Capped sense RNA was synthesized using the Ambion SP6 mMessage mMachine Kit according to manufacturer's instructions. For a $20 \mu \mathrm{l}$ total reaction volume, $1 \mu \mathrm{g}$ of linearized plasmid DNA was used. The transcription reaction was incubated for $2 \mathrm{~h}$ at $37^{\circ} \mathrm{C}$, followed by a 30 min DNA digest using $1 \cup$ Turbo DNase I. The synthesized sense RNA was purified using the lllustra RNAspin Mini kit (GE Healthcare) according to the manufacturer's instructions and was eluted in $30 \mu \mathrm{l}$ RNase free water at $80^{\circ} \mathrm{C}$. The RNA quality was checked on a $1 \%$ agarose gel and the RNA concentration was determined by loading $1 \mu \mathrm{l}$ of purified RNA on a NanoDrop 2000c spectrophotometer (Thermo Scientific). The RNA was stored in $2-4 \mu$ laliquots at $-80^{\circ} \mathrm{C}$.

\subsubsection{Xenopus methods}

Unless stated otherwise, standard $X$. laevis methods were done according to Sive et al. (2000)

$5 x$ MBS $440 \mathrm{mM} \mathrm{NaCl}, 5 \mathrm{mM} \mathrm{KCl}, 4.1 \mathrm{mM} \mathrm{MgSO}_{4}, 12 \mathrm{mM} \mathrm{NaHCO}_{3}$, $2.05 \mathrm{mM} \mathrm{CaCl}_{2}, 1.65 \mathrm{mM} \mathrm{Ca}\left(\mathrm{NO}_{3}\right)_{2}, 50 \mathrm{mM}$ HEPES, pH 7.8

Cysteine: $2 \%$ Cysteine- $\mathrm{HCl}$ in $0.1 \times \mathrm{MBS}, \mathrm{pH} 8$ Injection buffer: $2 \%$ Ficoll in $1 \times$ MBS 1000x Dexamethasone: $4 \mathrm{mg} / \mathrm{ml}$ dexamethasone in $100 \% \mathrm{EtOH}$

\subsubsection{Priming of $X$. laevis frogs}

Female frogs were stimulated by injecting $800 \mathrm{U}$ human chorionic gonadotropin (Ovogest, MSD animal health) into the dorsal lymph sac the 
evening before egg-laying. Primed frogs were kept overnight at $16{ }^{\circ} \mathrm{C}$. Egg laying started approximately $16 \mathrm{~h}$ after hCG priming.

\subsubsection{Preparation of the testis}

Male frogs were anesthetized by putting them for 30 min in narcotic $(1 \mathrm{~g} / \mathrm{l}$ tricaine methanesulfonate, $0.1 \% \mathrm{Na}_{2} \mathrm{HPO}_{4}, \mathrm{pH} 7$ ) (Guénette et al., 2013). The testis was isolated and stored at $4{ }^{\circ} \mathrm{C}$ in $1 \times$ MBS. Prior to usage, a testis lysate was made by macerating a small piece of testis in a small petri dish containing $1.5 \mathrm{ml} 1 \times$ MBS using a scalpel.

\subsubsection{In vitro fertilization}

By applying gentle pressure on the back of female frogs, eggs were collected in a petri-dish and fertilized by the addition of testis lysate 1:10 diluted with $\mathrm{H}_{2} \mathrm{O}$. Typically, between $100 \mu \mathrm{l}$ and $150 \mu \mathrm{l}$ of testis lysate were used. After 15$30 \mathrm{~min}$, the eggs were covered with $0.1 \times \mathrm{MBS}$ and stored at $12.5^{\circ} \mathrm{C}$.

\subsubsection{Microinjection}

Prior to the injection, the embryos were dejellied in a $2 \%$ cysteine solution and transferred to injection buffer. Injection needles were made by pulling small glass capillaries (GB100F-8P, Science Products $\mathrm{GmbH}$ ) in a needle-puller (PN-30, Science Products $\mathrm{GmbH}$ ). Needles were back-loaded with the desired injection mix and then attached to an microinjector PV $820(\mathrm{H}$. Sauer). Using a micrometer scale, the injection drop was adjusted to a final volume of $4 \mathrm{nl}$. The injection buffer was exchanged 1-2 $\mathrm{h}$ after injection with $0.1 \times \mathrm{MBS}$ and the injected embryos were cultivated at $12.5-18^{\circ} \mathrm{C}$ until the desired stage.

\subsubsection{Preparation of ectodermal explants (animal caps)}

Blastula staged embryos were transferred to a petri-dish coated with $1 \%$ agarose containing $0.8 \mathrm{x}$ MBS. The vitelline membrane was removed and explants of the animal cap were made using a gastromaster (Xenotech 
Engineering) with a $500 \mu \mathrm{m}$ yellow tip and "high" intensity. Animal caps were transferred to a fresh agarose-dish containing $0.8 \times \mathrm{MBS}$ and cultured at 12-18 ${ }^{\circ} \mathrm{C}$ until they reach the desired stage. To fix the caps, the embryos were placed in an Eppendorf tube and the buffer was removed. The embryos were frozen in liquid $\mathrm{N}_{2}$ and stored at $-80^{\circ} \mathrm{C}$.

\subsubsection{Dexamethasone treatment}

At the desired time (stage 10.5 for embryos or after excision of animal caps) dexamethasone in a final concentration of $4 \mu \mathrm{g} / \mathrm{ml}$ (from a 1000x stock solution) was added to the culture medium. The embryos or animal caps were cultured in the dark at $14-18{ }^{\circ} \mathrm{C}$ until they reached the desired stage (as determined from sibling control embryos). For collecting stage 14 animal caps or collecting early time points ( 3,6 or $9 \mathrm{~h}$ incubation time), caps were cultivated at $14^{\circ} \mathrm{C}$. For later time points $\left(12,15\right.$ or $25 \mathrm{~h}$ ), caps were cultivated at $18^{\circ} \mathrm{C}$. Animal caps were fixed by freezing in liquid $\mathrm{N}_{2}$ and were stored them at $-80^{\circ} \mathrm{C}$.

\subsubsection{Bimolecular Fluorescent Complementation assay (BiFC)}

BiFC (Saka et al., 2007) was used to visualize protein-protein interaction in vivo. Therefore, $500 \mathrm{pg}$ of each sense RNA encoding for the Vc- or Vn-fusion proteins was injected in both animal blastomeres at two cell stage $X$. laevis embryos. To label injected cells, 100 pg mRFP sense RNA was co-injected. At blastula stage (stage 8), the cells were dissociated by transferring the embryos to accutase (Sigma Aldrich) and culturing them for 5-6 h. Fluorescence microscopy was done by using an Axioplan2 fluorescent microscope (Zeiss) and pictures were taken using an Intas camera.

\subsubsection{In vitro synthesis of antisense RNA}

To prepare antisense RNA for whole mount in situ hybridizations (WMISH), a $25 \mu \mathrm{l}$ reaction containing $1 \mathrm{ng}$ linearized plasmid, 1x Transcription buffer (Fermentas), $1 \mathrm{mM} 1 \mathrm{mM}$ rATP (Boehringer), $1 \mathrm{mM}$ rGTP (Boehringer), $1 \mathrm{mM}$ rCTP (Boehringer), $0.64 \mathrm{mM}$ rUTPs (Boehringer), $0.34 \mathrm{mM}$ Digoxigenin-rUTP 
(Boehringer), $0.03 \mu \mathrm{M}$ DTT, $40 \mathrm{U}$ Ribolock RNase inhibitor (Fermentas), $30 \mathrm{U}$ T3 or SP6 RNA-Polymerase in RNase-free water was used. The in vitro transcription was incubated at $37^{\circ} \mathrm{C}$ for two hours. Afterwards, the template was digested by a $30 \mathrm{~min}$ incubation with $1 \mathrm{U}$ TurboDNase I (Ambion) at 37 ${ }^{\circ} \mathrm{C}$. Purification of the antisense RNA was done using the RNeasy Mini kit (Qiagen) according to manufacturer's instructions. The RNA was eluted two times with $50 \mu \mathrm{RNase}$-free water at $80^{\circ} \mathrm{C}$. The RNA quality was checked on a $1 \%$ agarose gel. Afterwards, the antisense RNA was diluted in $1 \mathrm{ml}$ of hybridization buffer (see in situ hybridization) and stored at $-20^{\circ} \mathrm{C}$.

\subsubsection{Whole mount in-situ hybridization (WMISH)}

10x MEM: $1 \mathrm{M}$ Mops, $20 \mathrm{mM}$ EGTA, $10 \mathrm{mM} \mathrm{MgSO}_{4}, \mathrm{pH} 7.4$ (stored in the dark) 10x PBS: $1.75 \mathrm{M} \mathrm{NaCl}, 1 \mathrm{M} \mathrm{KCl}, 65 \mathrm{mM} \mathrm{Na}_{2} \mathrm{HPO}_{4}, 18 \mathrm{mM} \mathrm{KH}_{2} \mathrm{PO}_{4}$, $\mathrm{pH} 7.4$

$\mathrm{K}_{3} \mathrm{FE}(\mathrm{CN})_{6}: 0.5 \mathrm{M}$ in $\mathrm{H}_{2} \mathrm{O}$ (stored in the dark)

$\mathrm{K}_{4} \mathrm{FE}(\mathrm{CN})_{6}: 0.5 \mathrm{M}$ in $\mathrm{H}_{2} \mathrm{O}$ (stored in the dark)

MEMFA: $4 \%$ (v/v) formaldehyde (37\%) in 1x MEM

X-Gal: $40 \mathrm{mg} / \mathrm{ml}$ 5-Bromo-4-chloro-3-indolyl-b-D-galactopyranoside in formamide (stored $-20^{\circ} \mathrm{C}$ in the dark)

X-Gal staining solution: $1 \mathrm{mg} / \mathrm{ml} \mathrm{X-Gal,} 5 \mathrm{mM} \mathrm{K}_{3} \mathrm{FE}(\mathrm{CN})_{6}, 5 \mathrm{mM}$

$\mathrm{K}_{4} \mathrm{Fe}(\mathrm{CN})_{6}, 2 \mathrm{mM} \mathrm{MgCl}_{2}$ in $1 \times$ PBS

PTw: $0.1 \%$ Tween-20 in $1 \times$ PBS

Proteinase K: $5 \mu \mathrm{g} / \mathrm{ml}$ proteinase $\mathrm{K}$ (Merck) in $0.1 \times$ PBS

Triethanolamine: $0.93 \mathrm{~g}$ triethanolamine in $50 \mathrm{ml} \mathrm{H}_{2} \mathrm{O}(0.1 \mathrm{M}), \mathrm{pH} 7.5$

PTw/FA: 4\% (v/v) formaldehyde in PTw

Hybridization Mix: 50\% formamide, $1 \mathrm{mg} / \mathrm{ml}$ Torula RNA, $10 \mu \mathrm{g} / \mathrm{ml}$ Heparin, 1x Denhardt's, 0.1\% Tween-20, 0.1\% CHAPS, 10 mM EDTA in 5x SSC 20X SSC: $3 \mathrm{M} \mathrm{NaCl}, 0.3 \mathrm{M} \mathrm{NaCitrate,} \mathrm{pH}$ 7.2-7.4

100x Denhardts solution: 2\% BSA 2\% Polyvinylpyrollidone (PVP), 2\% Ficoll RNAse Solution: $10 \mu \mathrm{g} / \mathrm{ml}$ RNAse A, $0.01 \mathrm{U} / \mathrm{ml}$ RNAse T1 in 2X SSC

MAB: $100 \mathrm{mM}$ maleic acid, $150 \mathrm{mM} \mathrm{NaCl}, \mathrm{pH} 7.5$

MAB/BMB: $2 \%$ BMB in $1 \times$ MAB

MAB/BMB/HS: $2 \% \mathrm{BMB}, 20 \%$ heat-treated horse serum in $1 \times \mathrm{MAB}$ 
APB: $100 \mathrm{mM}$ Tris- $\mathrm{HCl}, \mathrm{pH}$ 9.0, $50 \mathrm{mM} \mathrm{MgCl}, 100 \mathrm{mM} \mathrm{NaCl}, 0.1 \%$ Tween20, $\mathrm{pH} 9.0$

NBT: $100 \mathrm{mg} / \mathrm{ml}$ in $70 \%$ dimethylformamide, stored in the dark

BCIP: $50 \mathrm{mg} / \mathrm{ml}$ in 100\% dimethylformamide, stored in the dark

Staining solution: $80 \mu \mathrm{g} / \mathrm{ml}$ NBT, $175 \mu \mathrm{g} / \mathrm{ml} \mathrm{BCIP}$ in APB

Bleaching solution: $5 \%$ formamide, $1.5 \% \mathrm{H}_{2} \mathrm{O}_{2}$ in $0.5 \times \mathrm{SSC}$

WMISH was used to identify the endogenous temporal and special expression of RNA transcripts. WMISH was performed (Harland, 1991; Hollemann et al., 1996) with minor modifications. For detection, digoxigenin-labeled antisense RNA was used, and visualized using an a-dig antibody coupled to alkaline phosphatase. All steps, if not otherwise indicated, were performed at room temperature with mild shaking.

\subsection{Fixation and X-Gal staining}

For the identification of the injected side of the embryos and to analyze the distribution of the injected material, embryos were co-injected with $75 \mathrm{pg} \beta$-gal mRNA. Embryos were fixed in MEMFA in small glass vials for $20 \mathrm{~min}$, followed by washing three times with $1 \mathrm{x}$ PBS for $10 \mathrm{~min}$. To visualize the $\beta$-gal distribution, X-Gal staining was performed (Hardcastle et al., 2000). Embryos were incubated in X-Gal staining solution in the dark until the desired staining intensity (approx. between 20-30 min). Afterwards, the staining solution was removed and the embryos were washed three times with 1x PBS for 10 min each washing. Embryos were fixed in MEMFA for $25 \mathrm{~min}$ and then washed three times with $100 \%$ ethanol for 5 min. Embryos were stored at $-20{ }^{\circ} \mathrm{C}$ in $100 \%$ ethanol.

\subsection{Rehydration}

The embryos were rehydrated by several washing steps in which ethanol was stepwise replaced by $1 \times$ PBS (75\%, 50\%, 25\% ethanol). Following Rehydration, the embryos were washed four times with PTw for 5 min each washing. 


\subsection{Proteinase $\mathrm{K}$ treatment}

To make the cells of the embryo accessible for the antisense RNA, the embryos were permeabilized by proteinase $\mathrm{K}$ treatment. The duration of the treatment was dependent on the developmental stage of the embryo. Stage 28 embryos were treated for $17 \mathrm{~min}$ in $2 \mathrm{ml}$ proteinase $\mathrm{K}$ solution. The treatment was stopped by immediately proceeding with the acetylation.

\subsection{Acetylation}

Embryos were washes two times for 5 min with triethanolamine. To prevent nonspecific antisense RNA interaction, the embryos were acetylated by incubation with $25 \mu \mathrm{l}$ acetic anhydride in $4 \mathrm{ml}$ triethanolamine. The embryos were then washed two times for $5 \mathrm{~min}$ in PTw and refixed with PTw/FA for 20 $\min$. The fixation solution was removed by washing the embryos five times for 5 minutes with PTw.

\subsection{Hybridization reaction}

Embryos were pre-hybridized in Hybridization mix for $5 \mathrm{~h}$ at $65^{\circ} \mathrm{C}$. Afterwards, the embryos were incubated with Hybridization mix containing the antisense RNA overnight at $65^{\circ} \mathrm{C}$.

\subsection{Washings}

On the next morning, embryos were washed three times with 2x SSC for 15 $\min$ at $60{ }^{\circ} \mathrm{C}$. Non-hybridized antisense RNA was removed by RNase treatment for $60 \mathrm{~min}$ at $37^{\circ} \mathrm{C}$. The RNases were washed with $2 x$ SSC for 10 min at RT followed by two $30 \mathrm{~min}$ washes with $0.2 x \mathrm{SSC}$ at $60{ }^{\circ} \mathrm{C}$. For preparation of the $A B$ reaction, the embryos were washed two times with $M A B$ for $15 \mathrm{~min}$ at RT. 


\subsection{Antibody reaction}

Before the antibody reaction, several blocking steps were performed by incubating the embryos for $20 \mathrm{~min}$ in $\mathrm{MAB} / \mathrm{BMB}$, followed by incubation for 40 min of with $M A B / B M B / H S$. Alkaline phosphatase-coupled anti-Dig antibody (1:5000 in MAB/BMB/HS, Sigma-Aldrich) was used for detection. The $A B$ reaction took place overnight at $4{ }^{\circ} \mathrm{C}$.

\subsection{Staining reaction}

On the next day, the embryos were washed at least five times for $10 \mathrm{~min}$ in MAB, followed by two washes with freshly made APB for $5 \mathrm{~min}$ at $4{ }^{\circ} \mathrm{C}$. Afterwards, the embryos were stained in staining solution in the dark at $4{ }^{\circ} \mathrm{C}$. The staining reaction typically took between one and five days.

\subsection{Background removal and bleaching}

To stop the staining reaction and to reduce the background staining, the embryos were washed in $100 \%$ methanol until the wash solution is not stained anymore. The methanol was the stepwise removed by washing in $75 \%, 50 \%$ and $25 \%$ methanol. The embryos were fixed in MEMFA for $30 \mathrm{~min}$. To bleach the embryos, they were washed two times in 0.5x SSC and incubated in bleaching solution until the pigmentation was gone (typically between 1 and 4 h). The embryos were washes two times in 0.5x SSC and stored indefinitely in MEMFA.

\subsubsection{Agarose gel electrophoresis}

TAE (Tris-acetate-EDTA): 40 mM Tris-acetate, 2 mM EDTA, pH 8.5

Analysis of PCR products or DNA fragments was performed by agarose gel electrophoresis (Sharp et al., 1973). Agarose gels were made by melting a final concentration of $1-2 \%$ (depending on the expected size of the fragments) agarose powder (Roth) in TAE buffer. TAE buffer was used as running buffer. 
To determine the size of the DNA fragments, standard DNA ladders (Fast Ruler DNA ladder, Fermentas) were used. To visualize the DNA fragments, 30 $\mu \mathrm{g}(0.5 \mu \mathrm{g} / \mathrm{ml})$ ethidium bromide was added to the agarose gel. For documentation, a ChemiDoc (Bio-Rad) chamber using an Intas camera and software was used.

\subsubsection{PCR cloning}

For cloning, template DNA was amplified via PCR with the High Fidelity enzyme mix (Thermo Scientific) in a total reaction volume of $50 \mu \mathrm{l}$ reaction containing $1 \mathrm{x}$ High Fidelity Buffer with $15 \mathrm{mM} \mathrm{MgCl} 2,0.2 \mathrm{mM}$ of each dNTP (Thermo Scientific), $0.2 \mu \mathrm{M}$ of each primer, $0.02 \mathrm{U}$ High Fidelity PCR enzyme mix (Thermo Scientific) and $100 \mathrm{ng}$ template DNA. PCR was done in a thermocycler using the following settings: $5 \mathrm{~min}$ at $95^{\circ} \mathrm{C}, 45 \mathrm{~s}$ at $95^{\circ} \mathrm{C}, 45 \mathrm{~s}$ at $\mathrm{T}_{\mathrm{m}}$ of the primer $-2^{\circ} \mathrm{C}, 60 \mathrm{~s} / \mathrm{kb}$ length $72^{\circ} \mathrm{C}, 5 \mathrm{~min} 72^{\circ} \mathrm{C}$. Steps no $2-4$ were repeated in a various number of cycles ( $30-40$ cycles) depending on the primer pairs used.

\subsubsection{Gel purification of PCR and restriction fragments}

PCR fragments from PCR or restriction reactions, were isolated on an agarose gel, the desired fragment was excised and the purified by using the "GFX PCR DNA and Gel Band Purification" kit (GE Healthcare) according to the manufacturer's instruction.

\subsubsection{DNA restriction digestion}

Restriction digestion was performed using Fermentas Life Science restriction endonucleases according to manufacturer's instructions.

\subsubsection{Ligation}

Standard ligation reactions were performed using a $20 \mu$ reaction containing 1x T4 DNA Ligase buffer (Fermentas Life Science)., 1U T4 DNA ligases, 100 
ng linear vector DNA and insert DNA in a 1:1 to 5:1 ratio. The ligation reaction was incubated overnight at $16{ }^{\circ} \mathrm{C}$.

\subsubsection{Chemical transformation of bacterial cells}

Plasmid DNA was added to 100-200 $\mu$ l thawed chemical competent cells, and incubated on ice for $30 \mathrm{~min}$. Heat-shock was performed by incubating the cells for $60 \mathrm{~s}$ at $42^{\circ} \mathrm{C}$ for retransformation or $90 \mathrm{~s}$ at $42{ }^{\circ} \mathrm{C}$ for ligation reactions, followed by immediately cooling on ice for $3 \mathrm{~min}$. Afterwards, $800 \mu \mathrm{l}$ of LB medium was added to the cells, followed by incubation under constant shaking (300 rpm) for $30 \mathrm{~min}$ at $37^{\circ} \mathrm{C}$ for retransformation or $45 \mathrm{~min}$ at $37^{\circ} \mathrm{C}$ for cloning. The cells were then centrifuged for $30 \mathrm{~s}$ at $10,000 \mathrm{rpm}$ and $800 \mu \mathrm{l}$ of LBmedium was removed. The cells were resuspended in the remaining medium and plated on a LB agar plate containing the selective antibiotic and incubated overnight at $37^{\circ} \mathrm{C}$.

\subsubsection{Plasmid DNA preparation}

Bacteria were cultured in $4 \mathrm{ml}$ LB media together with the selective antibiotic overnight at $37^{\circ} \mathrm{C}$ with shaking at $250 \mathrm{rpm}$. For mini plasmid preparation, $3 \mathrm{ml}$ of the bacteria suspension was centrifuged for $2 \mathrm{~min}$ at 13,000 rpm. Plasmid DNA was isolated using the GeneJET Plasmid Miniprep kit (Fermentas) following manufacturer's instructions. For isolating preparative amounts (1 $\mu \mathrm{g} / \mu \mathrm{l})$ the NucleoBond Xtra Midi kit (Macherey-Nagel) was used according to the manufacturer's instruction. To quantify the concentration, $1 \mu \mathrm{l}$ of the DNA was applied on the NanoDrop 2000c spectrophotometer (Thermo Scientific).

\subsubsection{DNA sequencing}

DNA sequencing was performed by "SEQLAB Sequence Laboratories Göttingen $\mathrm{GmbH}$ ", according to the company's instructions using $1.2 \mu \mathrm{g}$ of DNA for sequencing. 


\subsubsection{ATAC sequencing}

Lysis buffer $10 \mathrm{mM}$ Tris- $\mathrm{HCl}, \mathrm{pH} 7.4,10 \mathrm{mM} \mathrm{NaCl}, 3 \mathrm{mM} \mathrm{MgCl} 2,0.1 \%$ Igepal CA-630, (can be stored at $4{ }^{\circ} \mathrm{C}$ for one week)

To identify open chromatin regions on the DNA, ATAC-sequencing was used. ATAC sequencing was performed after (Buenrostro et al., 2013) with modifications. For each sample 40 animal caps previously frozen in liquid $\mathrm{N}_{2}$ and stored at $-80^{\circ} \mathrm{C}$ were used.

\subsubsection{Transposition reaction}

Animal caps were carefully lysed in $100 \mu \mathrm{l}$ fresh ATAC-lysis buffer using a pestle, followed by centrifugation for $5 \mathrm{~min}$ at $2000 \mathrm{rpm}$ at $4{ }^{\circ} \mathrm{C}$. The supernatant was removed and the nuclei pellet resuspended on ice in a $50 \mu \mathrm{l}$ transposition solution containing 2x TD reaction buffer (Nextera DNA Library Prep kit, Illumina), $2.5 \mu$ TDE1 (Nextera DNA Library Prep kit, Illumina) and incubated for $30 \mathrm{~min}$ at $37{ }^{\circ} \mathrm{C}$. The transposition reaction was immediately stopped by purifying the DNA using the NucleoSpin Gel and PCR Clean-up kit (Macherey-Nagel) according to the manufacturer's instructions. The DNA was eluted in $10 \mu$ Elution buffer (10 $\mu \mathrm{M}$ Tris-HCl, $\mathrm{pH} 8)$.

\subsubsection{Library preparation}

Transposed DNA $(10 \mu \mathrm{l})$ was added to a total $50 \mu \mathrm{l}$ reaction mix containing $1.25 \mu \mathrm{M}$ PCR primer1 (Illumina), $1.25 \mu \mathrm{M}$ barcoded PCR primer2 (Illumina) and NEBNext High-Fidelity PCR Master mix. The samples were first amplified in the thermocycler using the following program: $5 \mathrm{~min}$ at $72^{\circ} \mathrm{C}, 30 \mathrm{~s}$ at $98^{\circ} \mathrm{C}$, followed by 5 cycles of $10 \mathrm{~s}$ at $98{ }^{\circ} \mathrm{C}, 30 \mathrm{~s}$ at $63^{\circ} \mathrm{C}$ and $1 \mathrm{~min}$ at $72{ }^{\circ} \mathrm{C}$. Afterwards, the appropriate number of PCR cycles was determined using qPCR by combining $5 \mu \mathrm{l}$ of the previously amplified DNA with a $10 \mu \mathrm{l}$ reaction containing, 1.25 $\mu \mathrm{M}$ PCR primer 1, $1.25 \mu \mathrm{M}$ PCR primer 2, 1x SYBR Green I and 1x NEBNext High-Fidelity PCR Master Mix. The qPCR was performed using a CFX96 ${ }^{\mathrm{TM}}$ Real-Time System (Bio-Rad) with the following program (30 
$\mathrm{s}$ at $98^{\circ} \mathrm{C}$, followed by 20 cycles of $10 \mathrm{~s}$ at $98^{\circ} \mathrm{C}, 30 \mathrm{~s}$ at $63^{\circ} \mathrm{C}$ and $1 \mathrm{~min}$ at $\left.72{ }^{\circ} \mathrm{C}\right)$. Afterwards, the remaining $45 \mu \mathrm{PCR}$ reaction was further amplified by $30 \mathrm{~s}$ at $98^{\circ} \mathrm{C}$, followed by a custom number of PCR cycles $\left(10 \mathrm{~s}\right.$ at $98^{\circ} \mathrm{C}, 30$ $\mathrm{s}$ at $63^{\circ} \mathrm{C}$ and $1 \mathrm{~min}$ at $72{ }^{\circ} \mathrm{C}$ ), where the number of remaining PCR cycles is the number of cycles that corresponded to one-third of maximum fluorescent intensity in the qPCR. The quality of the library was checked using the Bioanalyzer 2000c (Agilent).

\begin{tabular}{|l|l|}
\hline Sample & Number of PCR cycles \\
\hline CC 3 h replicate 1 & $10 x$ \\
\hline Ptf1a 3 h replicate 1 & $7 x$ \\
\hline CC 12 h replicate 1 & $16 x$ \\
\hline Ptf1a 12 h replicate 1 & $12 x$ \\
\hline CC 3h replicate 2 & $14 x$ \\
\hline Ptf1a 3 h replicate 2 & $16 x$ \\
\hline CC 12 h replicate 2 & $16 x$ \\
\hline Ptf1a 12 h replicate 2 & $12 x$ \\
\hline
\end{tabular}

Table 2.6 List of PCR cycles for each ATAC sample

\subsubsection{Sequencing (by TAL)}

Sequencing was performed by the University Medical Center Göttingen (UMG) -Transcriptome and Genome Analysis Laboratory (TAL) with the HiSeq 2500 in the $50 \mathrm{bp}$ paired end mode, with a density of 150,000,000 reads. Two biological replicates were used for each reaction. Sequencing quality was analyzed using the FastQ software (http://www.bioinformatics. babraham.ac.uk/projects/fastqc/).

\subsubsection{Sequencing alignment and processing (performed by TAL)}

The sequence reads were aligned to the $X$. laevis genome (J-strain v 9.2) (Bowes et al., 2007). Mapping was performed using Bowtie2 (version 2.5) (Langmead and Salzberg, 2012). For processing and filtering of the data SAMtools (version 1.1) (Li et al., 2009) was used. Picard (version 1.126) was 
then used to calculate the number and percentage of duplicates. The filtered BAM file was converted into a tagAlign file containing the genomic coordinates of each read using Bedtools (v2.20.1) (Quinlan and Hall, 2010).

Peaks were called using MACS2 (version 2.1.1.20160309) (Feng et al., 2012) using smoothing window 150 and $p$-value threshold of 0.01 for calling peaks. Peaks were merged a differential peak analysis was done using the DESeq2 package (version 1.16.1) (Love et al., 2014) within the R/Bioconductor environment (www.bioconductor.org). Peaks determined as significantly enriched were those with adjusted $p$-value $<0.1$.

\subsubsection{Gene expression analysis by semiquantitative RT-PCR or quantitative Nanostring analysis}

\subsubsection{Total RNA isolation from ectodermal explants and whole embryos}

$X$. laevis embryos (5) or ectodermal explants (50) were fixed in liquid nitrogen and stored at $80^{\circ} \mathrm{C}$. The animal caps or embryos were then lysed in $400 \mu \mathrm{l}$ preqGOLD TriFast reagent (Preqlab) using a 29-gauge syringe and incubated at room temperature for $10 \mathrm{~min}$. During that time, they were vortexed for $30 \mathrm{~s}$. Afterwards, $80 \mu$ of chloroform (Roth) was added followed by vortexing for 30 $\mathrm{s}$ and $10 \mathrm{~min}$ centrifugation at $13,000 \mathrm{rpm}$ at $4{ }^{\circ} \mathrm{C}$. The upper phase $(200 \mu \mathrm{l})$ was transferred into a new eppendorf tube and then mixed with 1 volume (200 ul) chloroform by vortexing for $30 \mathrm{~s}$. After centrifugation for $5 \mathrm{~min}$ at 13,000 rpm and $4{ }^{\circ} \mathrm{C}$, the upper phase $(200 \mu \mathrm{l})$ was transferred into a new eppendorf tube and 1 volume of 2-propanol was added. After mixing, precipitation took place at $-20{ }^{\circ} \mathrm{C}$ for at least $2 \mathrm{~h}$. Afterwards, the precipitated RNA was centrifuged for $30 \mathrm{~min}$ at $4{ }^{\circ} \mathrm{C}$. The supernatant was removed and the pellet was washed with $400 \mu \mathrm{l} 75 \%$ ethanol. The air-dried pellet was dissolved in $12.5 \mu \mathrm{l}$ RNase-free water and DNase digest using 0,75 U DNase I (Thermo Scientific) was performed for $1.5 \mathrm{~h}$ at $37^{\circ} \mathrm{C}$. DNase activity was stopped by incubation at $70^{\circ} \mathrm{C}$ for $10 \mathrm{~min}$. The RNA concentration was determined on the NanoDrop 2000c spectrophotometer (Thermo Scientific). To check for genomic DNA contamination, a control PCR with $100 \mathrm{ng}$ RNA using primer for 
the housekeeping gene ornithine decarboxylase (odc) was performed and analyzed on a $2 \%$ agarose gel.

\subsection{Reverse transcription}

To synthesize $10 \mu \mathrm{l}$ cDNA, $100 \mathrm{ng}$ of total RNA was used in a reaction mix containing 1x Go Taq flexi buffer (Promega), $5 \mathrm{mM} \mathrm{MgCl}_{2}$ (Fermentas), 2.5 $\mathrm{mM}$ random hexamer (Invitrogen), $1 \mathrm{mM}$ dNTP mix (Thermo Scientific), 0,16 U Ribolock RNase inhibitor (Thermo Scientific), and 0,8 U MulV reverse transcriptase (Roche). The reaction took place in a thermocycler (Biometra) using the following program: $20 \mathrm{~min}$ at $20^{\circ} \mathrm{C}, 1 \mathrm{~h}$ at $42^{\circ} \mathrm{C}, 5 \mathrm{~min}$ at $95^{\circ} \mathrm{C}$.

\subsection{Reverse transcription-PCR (RT-PCR)}

Semiquantitative RT-PCR was performed using a total volume of $12.5 \mu \mathrm{l}$ containing 1x GoTaq green reaction buffer (Promega), $0.2 \mu \mathrm{M}$ of both forward and reverse primer and 0.5 units GoTaq DNA polymerase (Promega). For each reaction $2.5 \mu \mathrm{l}$ cDNA was used as a template for PCR. Amplification took place in a thermocycler (Biometra) using the following program: 2 min at $95^{\circ} \mathrm{C}$, $45 \mathrm{~s}$ at $95^{\circ} \mathrm{C}, 45 \mathrm{~s}$ at $\mathrm{Tm}$ of the primer $-2^{\circ} \mathrm{C}, 45 \mathrm{~s}$ at $72{ }^{\circ} \mathrm{C}, 5 \mathrm{~min}$ at $72{ }^{\circ} \mathrm{C}$. Steps no 2-4 were repeated in a various number of cycles ( 25-35 cycles), which is dependent on the specific primer pairs, before proceeding with step 5. RT-PCR reactions were analyzed on a $2 \%$ agarose gel as described above.

\subsubsection{Quantitative Nanostring analysis}

Quantitative analysis was performed using $700 \mathrm{ng}$ of total RNA on the Nanostring digital multiplexed expression analysis system. The analyzed genes as well as the target region are shown in the appendix. Data analysis was performed by using the nSolver software (version 2.5). For normalization, the counts were first normalized to the mean of the positive control counts. Afterwards, the counts were normalized for the geometric mean of the housekeeping genes odc, rplp0 and ppi1l. If several developmental stages were analyzed at the same time, normalization was performed to the geometric 
mean of the housekeeping gene ppi1l only. Background subtraction was performed by subtracting the mean value and two times the standard deviation of the eight negative control counts for each lane. Negative values were set to 1. Data from three independent experiments ( $A, B$ and $C)$ were analyzed.

\subsubsection{RNA sequencing}

\subsubsection{RNA isolation}

RNA isolation for RNA sequencing was performed similar to isolating RNA for RT-PCR with the following adjustments to the protocol:

RNA from 50 animal caps was extracted as described above until the first step of precipitation with isopropanol. Afterwards, the precipitated RNA was centrifuged for $30 \mathrm{~min}$ at $4{ }^{\circ} \mathrm{C}$. The supernatant was removed and the pellet was washed with $400 \mu \mathrm{l} 75 \%$ ethanol. The air-dried pellet was dissolved in 47 $\mu \mathrm{R}$ Rase-free water. DNA digest was performed by incubating the isolated RNA for $1 \mathrm{~h}$ at $37^{\circ} \mathrm{C}$ in a $50 \mu$ total reaction volume containing $1 \times$ DNase I reaction buffer (Thermo Scientific), $1 \mathrm{U}$ DNase I (Thermo Scientific) and $0.4 \mathrm{U}$ RNase inhibitor (Thermo Scientific). DNA digest was stopped by the addition of $150 \mu \mathrm{l}$ RNase-free water. Phenol-chloroform-isoamylalcohol (Roth) (200 $\mu \mathrm{l})$ was added and the samples were vortexed for $30 \mathrm{~s}$, followed by $10 \mathrm{~min}$ centrifugation at $13,000 \mathrm{rpm}$ at $4^{\circ} \mathrm{C}$. To the upper phase $(200 \mu \mathrm{l}), 1$ volume $(200 \mu \mathrm{l})$ of chloroform-isoamylalcohol (ratio 24:1) was added. After vortexing for $30 \mathrm{~s}$, the samples were centrifuged for $5 \mathrm{~min}$ at $13,000 \mathrm{rpm}$ and $4{ }^{\circ} \mathrm{C} .1 / 10$ volume $(20 \mu \mathrm{l})$ ammonium acetate and 1 volume $(200 \mu \mathrm{l})$ 2-propanol were added and incubated for at least $2 \mathrm{~h}$ at $-20^{\circ} \mathrm{C}$. The precipitate was centrifuged for $30 \mathrm{~min}$ at $13,000 \mathrm{rpm}$ at $4{ }^{\circ} \mathrm{C}$ and then washed by $400 \mu \mathrm{l}$ of $75 \%$ ethanol. The RNA pellet was then dissolved in $20 \mu \mathrm{R}$ Rase-free water. To check for genomic DNA contamination, a control PCR with 100 ng RNA using oligonucleotides for housekeeping gene ornithine decarboxylase (odc) was performed and analyzed on a $2 \%$ agarose gel. RNA quality was checked by use of the 2100 Bioanalyzer (Aglient). 


\subsubsection{Sample preparation and sequencing}

Library preparation and sequencing was performed by the University Medical Center Göttingen (UMG) - Transcriptome and Genome Analysis Laboratory (TAL). Libraries were prepared with the TruSeq RNA Sample Prep Kit v2 (Illumina) following the manufacturer's instructions. Sequencing was performed with the HiSeq 2500 in the 50 bp single end mode. Two or three biological replicates were used for each reaction. Sequencing quality was analyzed using the FastQ software (http://www.bioinformatics.babraham. ac.uk/projects/fastqc/).

\subsubsection{Sequencing alignment (performed by TAL)}

The sequence reads were aligned to the $X$. laevis genome (J-strain $\vee$ 9.2) (Bowes et al., 2007). Mapping was performed using the software the STAR alignment software (ver. 2.5) (Dobin et al., 2013).

\subsubsection{Statistical analysis (performed by TAL)}

Normalization of the library samples and the differential gene analysis was performed using the DESeq2 package (version 1.16.1) (Love et al., 2014) within the R/Bioconductor environment (www.bioconductor.org). Candidate genes had a two-fold change induction compared to control samples and a FDR-corrected $p$-value of $<0.05$. 


\section{Results}

\subsection{Mutation of a single threonine residue in the Ptf1a C2 domain is sufficient to induce a mixed induced neuronal transmitter phenotype}

Similar to many bHLH proteins, Ptf1a contains multiple S/T-P residues, which are putative targets of cdk-dependent phosphorylation (Ubersax and Ferrell, 2006; Richts, 2013). Preventing of phosphorylation at those sites through mutation in Neurog2 leads to an increase in activation of the pan-neuronal marker tubb2b (Ali et al., 2011). To evaluate the role of such phosphorylation sites in Ptf1a activity, a mutant of the mouse Ptf1a in which all eight serine and threonine residues were mutated to alanine $\left(\mathrm{mPtf}^{\mathrm{a}} \mathrm{a}^{8 \mathrm{~S} / \mathrm{T} \rightarrow \mathrm{A}}\right)$ was evaluated in gain of function studies using pluripotent $X$. laevis embryonic cells (animal cap explants) in comparison to the wild-type Ptf1a. In contrast to Neurog2, no increase in neuronal-inducing activity of by the $P t f 1 a^{8 \mathrm{~S} / \mathrm{T} \rightarrow \mathrm{A}}$ mutant was observed. However, in addition to its ability to induce markers for a GABAergic cell fate, the Ptf1 $\mathrm{a}^{8 \mathrm{~S} / \mathrm{T} \rightarrow \mathrm{A}}$ mutant also induced the glutamatergic marker gene tIx3, a gene that is normally repressed by Ptf1a (Richts, 2013). Mutating the alanine residue at position 299 back to threonine $\left(\mathrm{mPtf} 1 \mathrm{a}^{7 \mathrm{~S} \rightarrow \mathrm{A}}\right)$ abolished the tlx3 induction (Richts, 2013), suggesting that the mutation of the T299 was responsible for this mixed neuronal transmitter phenotype activity (Richts, 2013).

To investigate if the mixed neuronal subtypes activity of the Ptf1a threonine mutant is conserved and is indeed sufficient to induce a mixed neuronal phenotype, the $X$. laevis variant $\left(\mathrm{Ptf}_{1} \mathrm{a}^{\mathrm{T} 243 \mathrm{~A}}\right)$ of the mouse $\mathrm{Ptf}^{1} \mathrm{a}^{\mathrm{T} 299 \mathrm{~A}}$ was created (Fig. 3.1A and B). The activity of this construct was compared with the wild-type Ptf1a, which promotes GABAergic markers and represses glutamatergic markers, as well as with the Rbpj-binding deficient mutant (Ptf1aW224A/W242A), which promotes a glutamatergic fate (Fig. 3.1A and B) (Hedderich, 2008; Hori et al., 2008; Hedderich, 2012; Hanotel et al., 2014) as the mutated threonine residue in the $\mathrm{Ptf}_{1} \mathrm{a}^{\mathrm{T} 243 \mathrm{~A}}$ mutant is directly adjacent to the Rbpj binding tryptophan residue within the Ptf1a C2 domain (Fig. 3.1B) The wild-type and mutated versions of Ptf1a were fused to the ligand binding 
domain of the glucocorticoid receptor (GR) (Gammill and Sive, 1997) to allow control over protein activity. In one blastomere at the two-cell, $20 \mathrm{pg}$ mRNA encoding the Ptf1a-fusion proteins was injected together with $75 \mathrm{pg}$ of $\beta$ galactosidase mRNA to allow identification of the injected side of the embryo. The protein activity was induced via dexamethasone treatment at gastrula stage (stage 10.5) and at tailbud stage (stage 28), embryos were collected and whole mount in-situ hybridization against the pan-neuronal marker tubb2b, the GABAergic marker gad1a, and the glutamatergic marker $t / x 3$ performed.

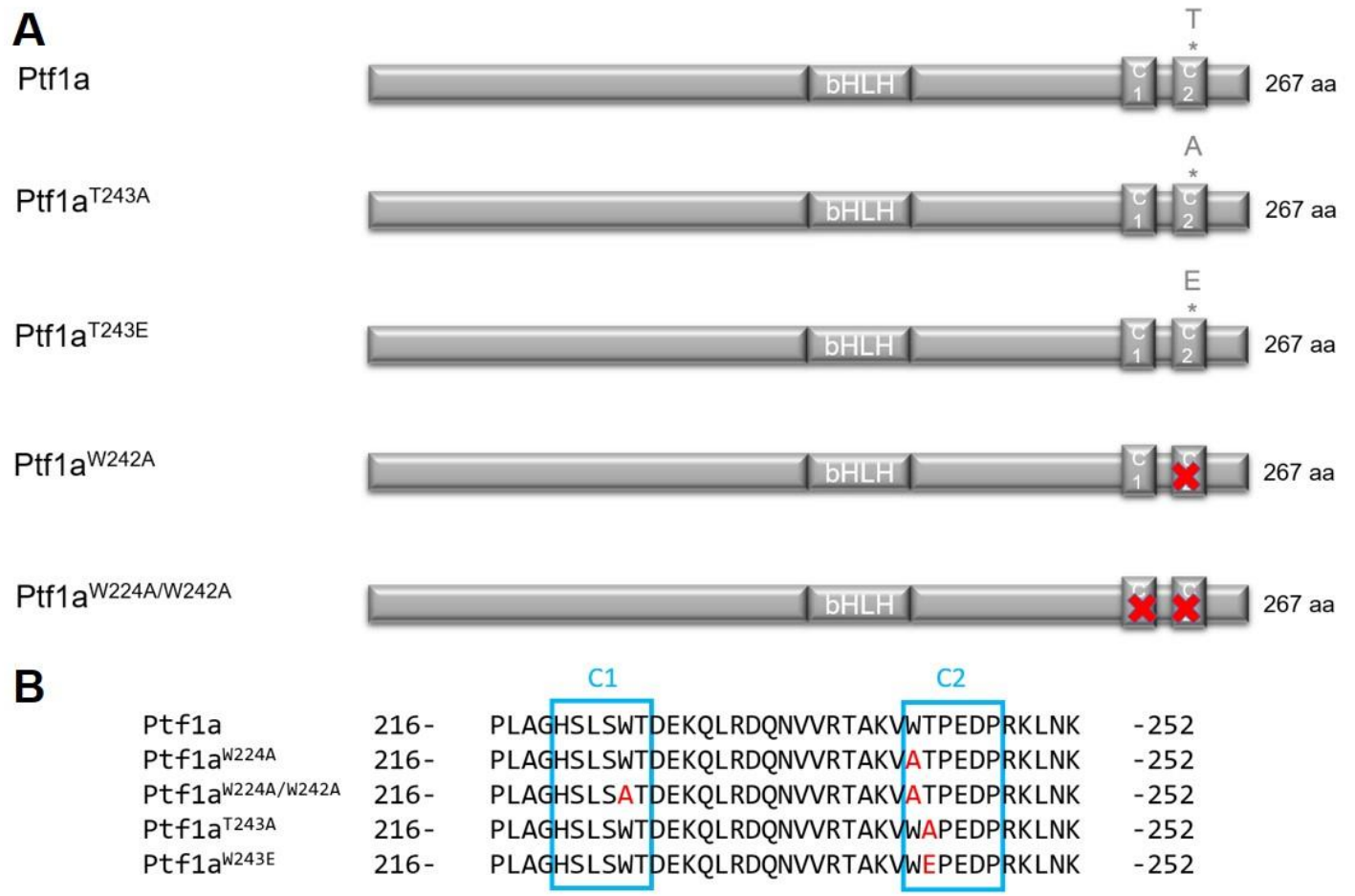

\section{Figure 3.1: Overview $X$. laevis Ptf1a mutants}

(A) Schematic overview over the Ptf1a constructs used for overexpression studies. Grey asterisks highlight the position of a mutated T-P site in the Ptf1a C2 domain. Red crosses indicate a mutation in a conserved Rbpj binding site. (B) Alignment of the highly conserved Ptf1a C1 and C2 domains of all Ptf1a constructs. The C1 and C2 domain are highlighted in blue, mutated residues are highlighted in red.

As shown in Figure 3.2B and consistent with previous reports, overexpression of wild-type Ptf1a promotes ectopic expression of $t u b b 2 b$ and gad $1 a$, while $t / x 3$ expression was suppressed. Ptf1 a ${ }^{\text {W224AW242A }}$ also induces ectopic expression of $t u b b 2 b$, but these ectopic neurons express $t / \times 3$ (Hedderich, 2008; Hedderich, 2012). 
A

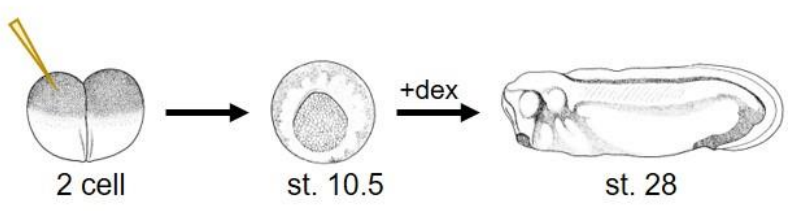

B
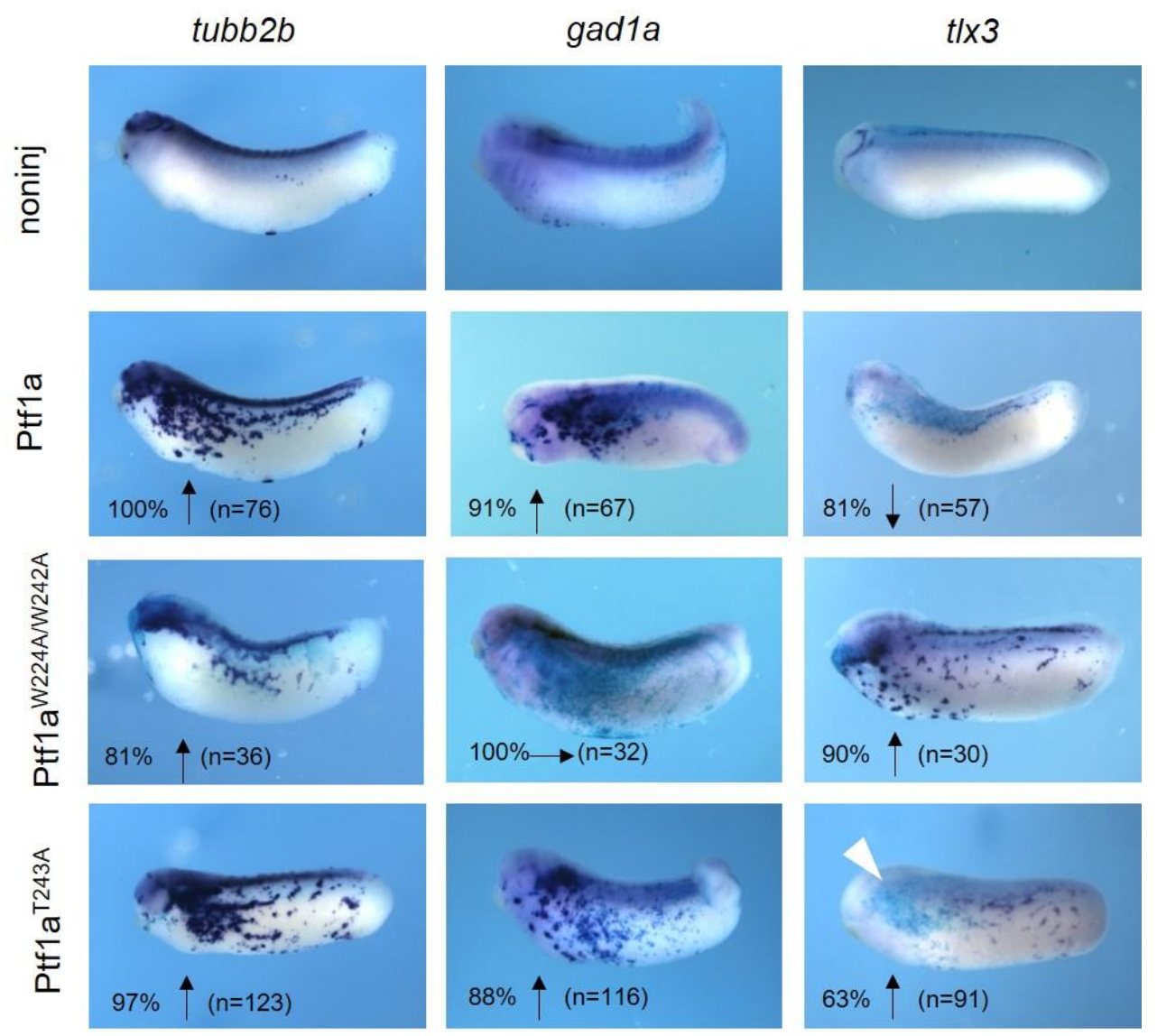

Figure 3.2: Ptf1a $\mathrm{a}^{\mathrm{T} 243 \mathrm{~A}}$ mutant induces a mixed neuronal transmitter phenotype in embryos

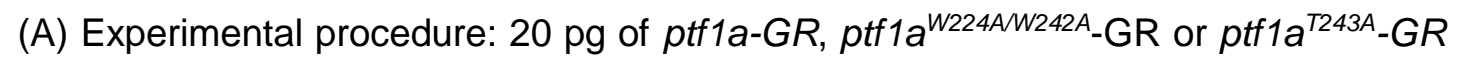
mRNA were injected in one animal blastomere at two-cell stage. For recognition of the injected side $\beta$-galactosidase RNA was co-injected. Protein activity was induced by addition of dexamethasone at stage 10.5. Embryos were collected for WMISH at stage 28. (B) Overview of WMISH for neuronal differentiation (tubb2b), GABAergic (gad1a) or glutamatergic $(t / x 3)$ cell fate of embryos overexpressing Ptf1a-GR,

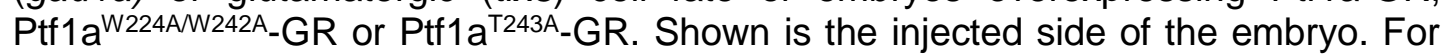
comparison non-injected embryos were shown at the top. Percentages refer to the total number of embryos showing the shown phenotype in relation to the total numbers of embryos showing $\beta$-gal expression (n). Black arrows refer to the induced phenotype (upregulation, downregulation or not affected). White arrowhead highlights the area of downregulation of endogenous $t / x 3$ expression by the Ptf $1 a^{T 243 A}$ mutant.

In contrast, overexpression of Ptf1 $\mathrm{a}^{\mathrm{T} 243 \mathrm{~A}}$ leads to an activation of all three markers, supporting our previous observations with the mouse Ptf1a ${ }^{\top 299 A}$ mutant. Interestingly, overexpression of Ptf1 $\mathrm{a}^{\mathrm{T} 243 \mathrm{~A}}$ still results in a repression 
of the endogenous $t / x 3$ expression on the injected side of the embryo. However, ectopic $t / x 3$ expression is now observed (Fig3.2B).

A

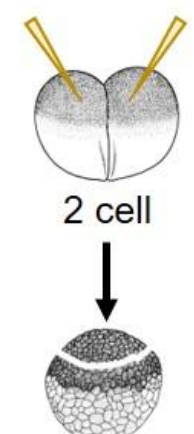

st. $8-9$

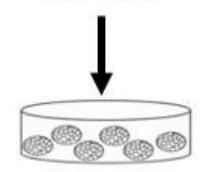

$+\operatorname{dex}\lfloor 25 \mathrm{~h}$
B

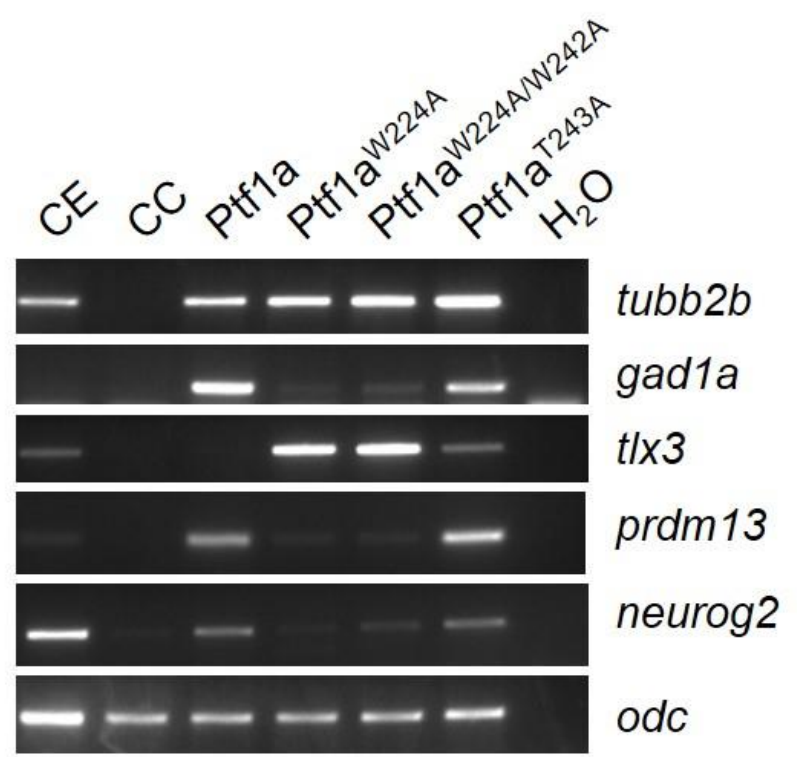

RT-PCR

Figure 3.3 Ptf1a ${ }^{\mathrm{T} 243 \mathrm{~A}}$ mutant induces a mixed neuronal transmitter phenotype in ectopic explants

(A) Experimental procedure: 20 pg of ptf1a-GR, ptf1a $a^{\text {W42A }}-G R$, ptf1 $a^{\text {W224A/W242A }}-G R$ or ptf1 ${ }^{T 243 A}-G R$ mRNA were injected in animally in both blastomeres of two-cell stage embryos. At blastula stage, animal caps were excised and protein activity induced by dexamethasone treatment for $25 \mathrm{~h}$. (B) RT-PCR analysis against markers for neuronal differentiation (tubb2b), GABAergic (gad1a) or glutamatergic ( $t / x 3$ ) cell fate, as well as two target genes for the trimeric Ptf1a complex (prdm13, neurog2). The housekeeping gene odc was used as a loading control. As additional controls, cDNA from total RNA isolations of noninjected control caps (CC) and non-injected control embryos (of the same developmental stage) were analyzed.

As the mutated threonine residue in the Ptf1 $1 \mathrm{a}^{\mathrm{T} 243 \mathrm{~A}}$ mutant is located adjacent to a known Rbpj binding site (Beres et al., 2006) in the Ptf1a C2 domain (Fig. 3.1B), a comparison of the overexpression phenotype between the Ptf1 $\mathrm{a}^{\mathrm{T} 243 \mathrm{~A}}$ and the Ptf1a ${ }^{\mathrm{W} 242 \mathrm{~A}}$ mutants was performed by injection $20 \mathrm{pg}$ of ptf1a-GR, ptf1aW242A-GR ptf1a $^{W 224 A} /{ }^{242 A}-G R$ or ptf1a ${ }^{T 243 A}-G R$ mRNA in the animal hemisphere of both blastomeres at two-cell stage. At blastula stage, the animal caps were excised and cultured for $25 \mathrm{~h}$ in the presence of dexamethasone to induce protein activity. Afterwards, RNA was isolated and semi-quantitative 
RT-PCR was performed against tubb2b, t/x3 and gad1a expression (Fig. 3.3A). As expected, overexpression of all four constructs promotes neuronal differentiation as shown by the increase in tubb2b expression (Fig. 3.3B). While the wild-type Ptf1a leads to an induction of gad1a, but not $t / x 3$, the Ptf1 $\mathrm{a}^{\mathrm{W} 224 \mathrm{~A} W 242 \mathrm{~A}}$ and Ptf1 $\mathrm{a}^{\mathrm{W} 242 \mathrm{~A}}$ mutants strongly induce $t / x 3$ but not gad1a. In contrast, overexpression of Ptf1a ${ }^{\mathrm{T} 243 \mathrm{~A}}$ induces both gad1a and $t / x 3$ (Fig. 3.3B), indicating that the phenotype is specific for the mutation in the $T_{243}$ residue.

To better understand the differential gene induction by the wild-type Ptf1a and

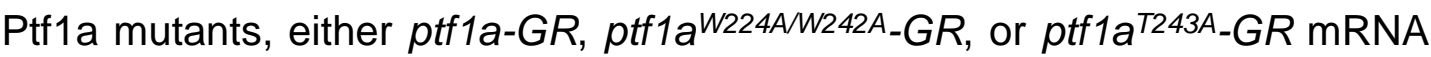
$(20 \mathrm{pg})$ were injected in the animal hemispheres of both blastomeres of twocell stage $X$. laevis embryos. At blastula stage, the animal caps were excised and cultured in the presence of dexamethasone for $25 \mathrm{~h}$ to induce the protein activity. Afterwards, RNA was isolated and quantitative Nanostring analysis was performed using a codeset containing known Ptf1a dimeric and trimeric target genes, as well as markers of neuronal differentiation and subtype specification (Fig. 3.4A).

All three Ptf1a constructs were able to upregulate markers for pan-neuronal differentiation such as tubb2b, myt1, ebf2 or dll1. (Fig. 3.4C-E; Table S1). Consistent with previous observations, the wild-type Ptf1a but not Rbpj-binding deficient mutant (Ptf1a ${ }^{\text {W224AW242A }}$, upregulated genes associated with the development of a GABAergic cell fate such as gad1a and prdm13, while Ptf1a ${ }^{\text {W224AW242A }}$ strongly upregulated $t / \times 3$ expression, which was not activated by the wild-type Ptf1 a (Fig. 3.4F-H). As previously demonstrated, the Ptf1a ${ }^{\text {T243A }}$ mutant upregulated markers associated with a GABAergic cell fate such as gad1a, prdm13, slc32a1 and pax2 as well as $t / x 3$ expression (Fig. 3.4F-H; TableS1). Comparing the expression levels induced by the different Ptf1a constructs showed that the strength of induction by the Ptf1 $1 \mathrm{a}^{\mathrm{T} 243 \mathrm{~A}}$ mutant of GABAergic marker genes such as prdm13 and gad1a was lower than for the wild-type Ptf1a (Fig. 3.4G, H). Moreover, the induction of $t / x 3$ expression was weaker than that induced by Ptf1aW224AW242A (Fig. 3.4F), highlighting the mixed identity of the Ptf1a ${ }^{T 243 A}$ mutant. Interestingly, the levels of activation of genes

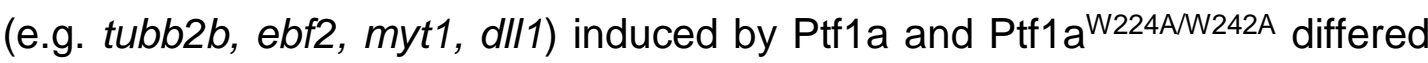
(Fig. 3.4C-E, Table S1). Those markers showed a weaker induction by the 
A
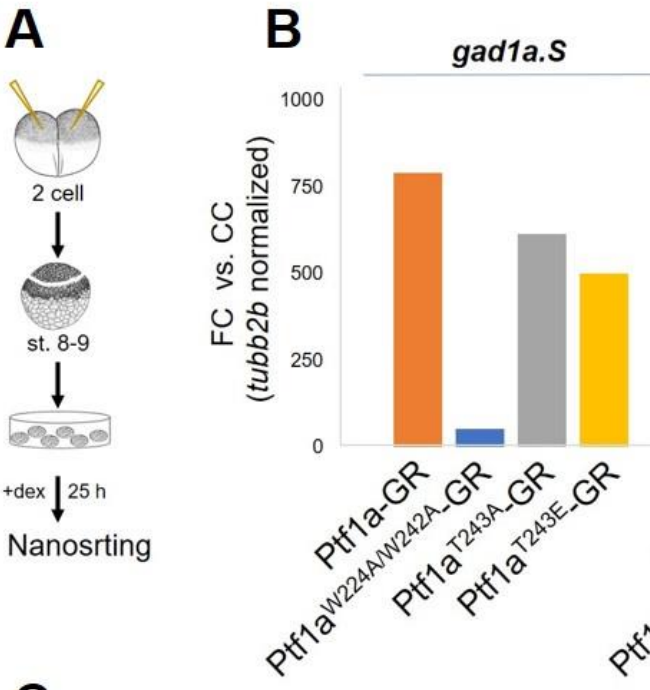

C

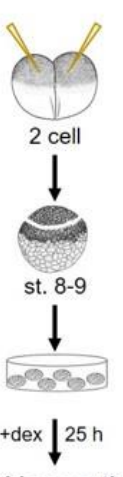

tubb2b.S

t/x3.L

prdm13.S

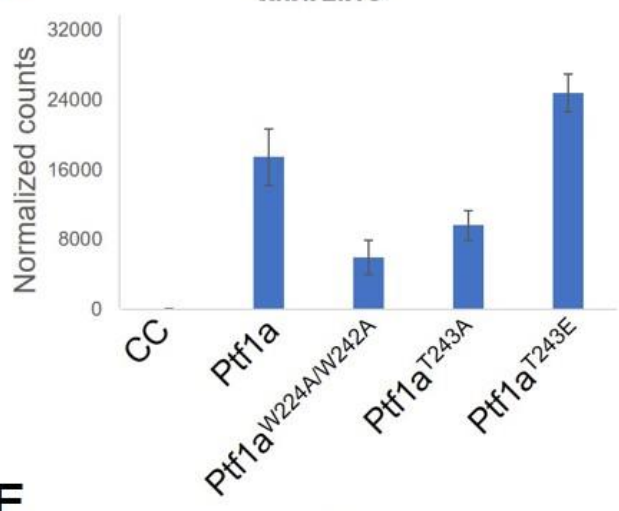

E

ebf2.s

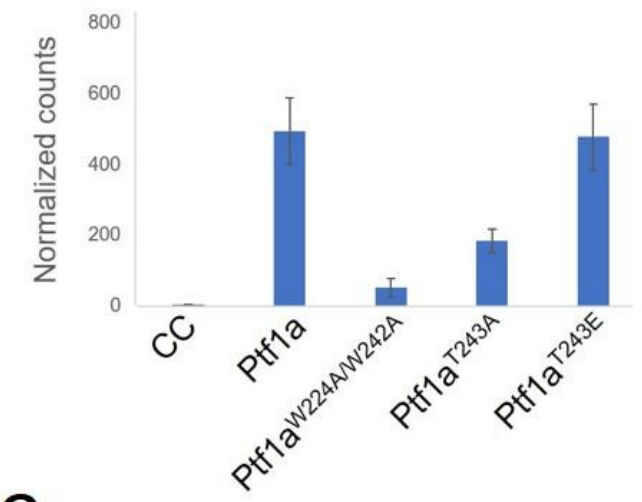

G
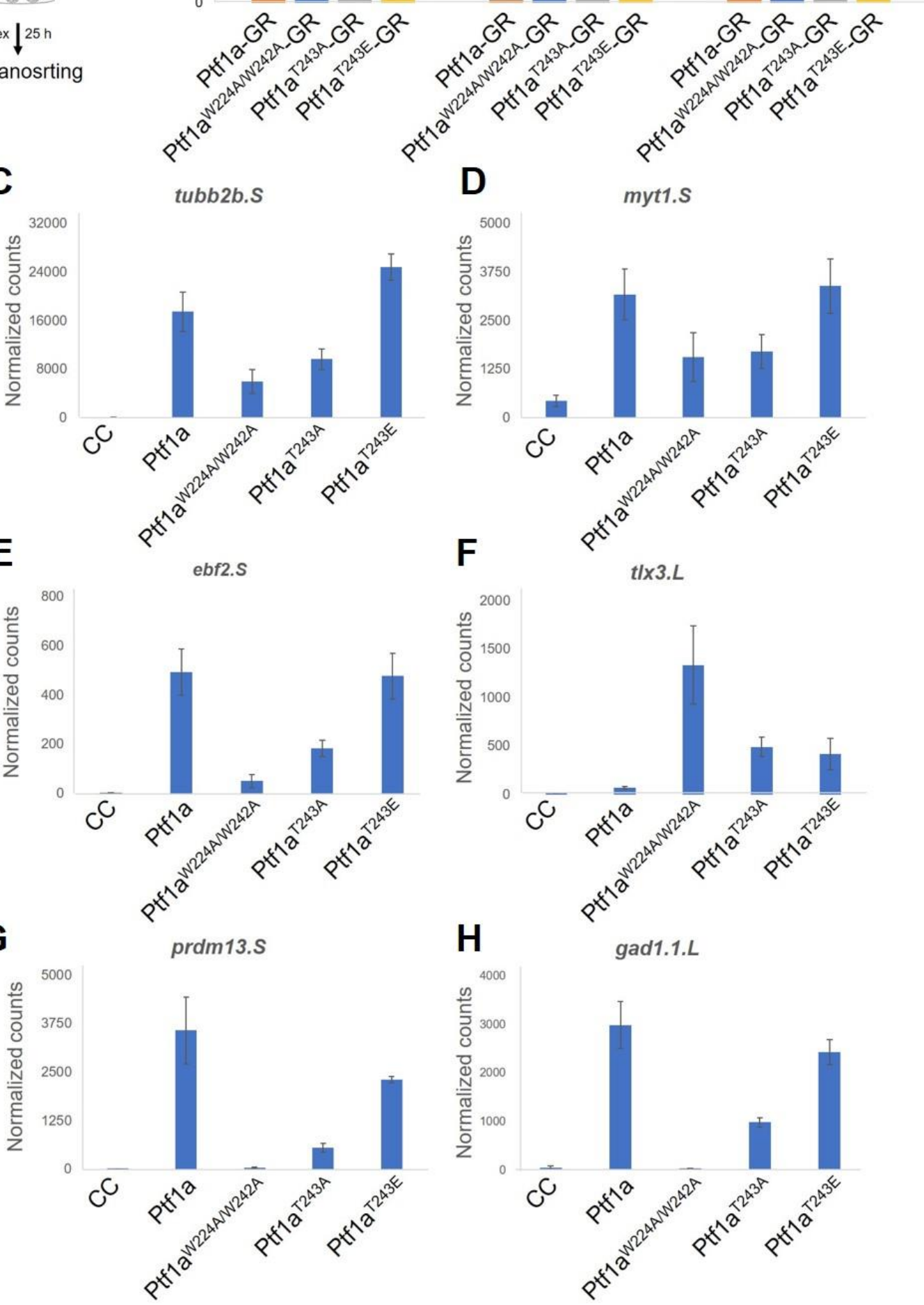

D
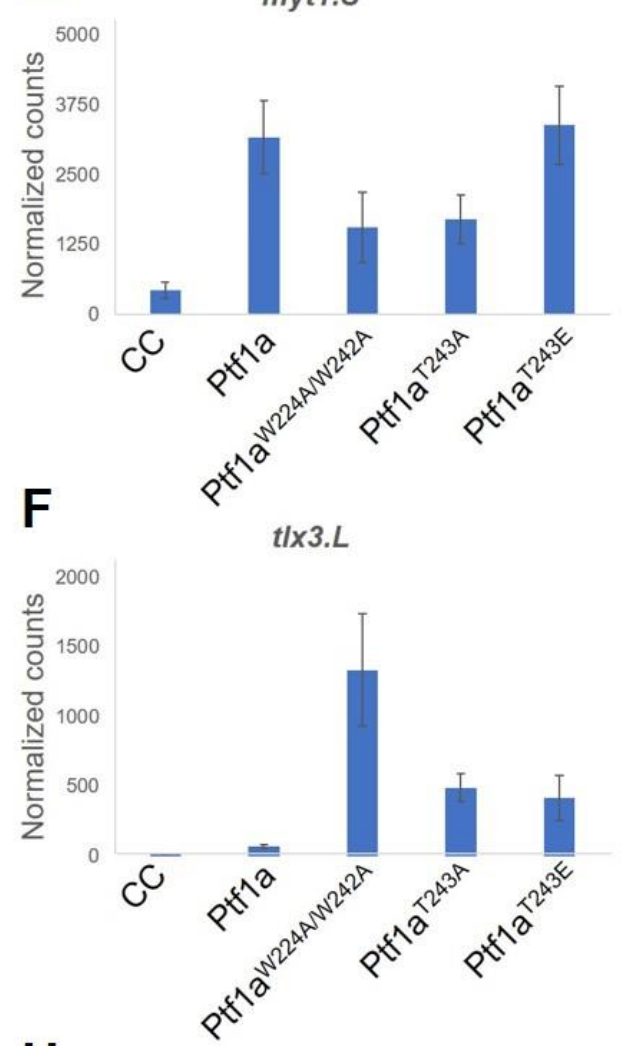

H

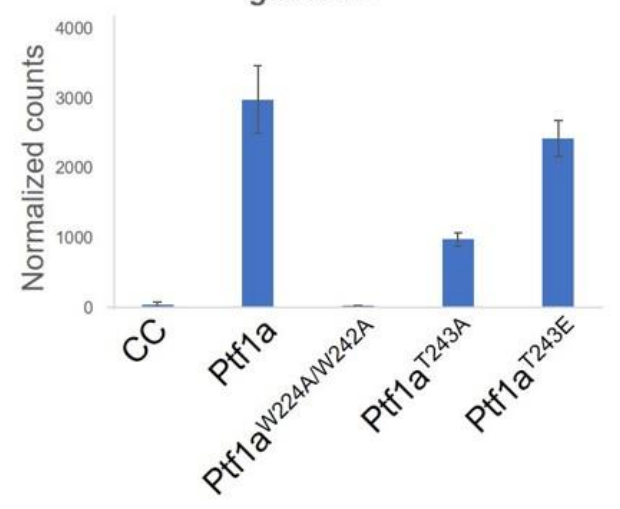




\section{Figure 3.4: Activities of distinct Ptf1aT 243 mutants}

(A) Experimental procedure: $20 \mathrm{pg}$ of ptf1a-GR, ptf1a ${ }^{\text {T243E }}-G R$, ptf1a ${ }^{\text {W224A.W242A }}-G R$ or ptf1a ${ }^{T 243 A}-G R$ mRNA were injected in both animal blastomeres of two-cell stage. At blastula stage, animal caps were excised. Protein activity was induced by dexamethasone treatment for $25 \mathrm{~h}$. (B) Shown is the average fold-change induction over non-injected control caps from three individual replicates. Data were normalized for the induction of tubb2b. The gene analyzed can be found on the $x$-axis $(C-H)$ Results of the quantitative Nanostring analysis. Shown are the average expression levels of marker genes from three biological replicates. Error bars refer to the standard error of the mean. Data were normalized for the three housekeeping genes odc, ppi1l and rplp0. .S and .L refer to the corresponding $X$. laevis homeolog targeted by the Nanostring probe. Note the different scales in each diagram.

Ptf1a ${ }^{\text {W224A/W242A }}$ and the Ptf1 $\mathrm{a}^{T 243 \mathrm{~A}}$ mutants, compared to the wild-type Ptf1a. These differences between versions of Ptf1a constructs were detected consistently in all three replicates (Table S2). To exclude that the differences in the induction of subtype specific marker genes gad1a and $t / x 3$ had their cause in a different efficiency of neuronal differentiation, the Nanostring data were normalized for tubb2b and the fold-change induction by wild-type and mutant Ptf1a compared to non-injected control caps. Considering the tubb2b levels, the differences in the induction of gad1a between Ptf1a and Ptf1a ${ }^{\mathrm{T} 243 \mathrm{~A}}$ was lower, but still significant. Moreover, normalization to tubb2b did not alter the observed gene induction by the different Ptf1a constructs, as gad1a was still induced by Ptf1a and Ptf1a ${ }^{T 243 A}$ but not by Ptf1aW224A/W242A, while $t / x 3$ expression was only induced by Ptf1a ${ }^{\text {W224AW242A }}$ and Ptf1a ${ }^{T 243 A}$ (Fig. 3.4B).

\subsection{Introducing a negative charge at the $\mathrm{Ptf1aT}_{243}$ residue induces a more wildtype Ptf1a like phenotype}

As the mutated threonine residue in the Ptf1 $\mathrm{a}^{\mathrm{T} 243 \mathrm{~A}}$ is a putative target of cell cycle dependent phosphorylation, a phosphomimic mutant was created in which threonine 243 was exchanged for glutamate (Ptf1a $\left.{ }^{\mathrm{T} 243 \mathrm{E}}-\mathrm{GR}\right)$ and analyzed in analogous gain of function assays (Fig. 3.1).

Similar to the wild-type Ptf1a and the Ptf1a mutants, the Ptf1a ${ }^{T 243 E}$ mutant induced pan-neuronal differentiation markers (myt1, zc3h12c, tubb2b, ebf2, dll1), but the strength of induction was similar to the wild-type Ptf1a with the 
other mutants inducing a much lower induction (Fig. 3.4, Table S1). In addition, the strength of the GABAergic markers genes (gad1a and prdm13) upregulation was much stronger for the phosphomimetic Ptf1 $\mathrm{a}^{\mathrm{T} 243 \mathrm{E}}$, compared to the phosphorylation-null mutant (Ptf1 $\mathrm{a}^{\top 243 \mathrm{~A}}$ ), further indicating this mutant behaved more like the wild-type Ptf1a (Fig. 3.4G, H). However, both Ptf1 $\mathrm{a}^{\mathrm{T} 243 \mathrm{E}}$ and Ptf1 $\mathrm{a}^{\mathrm{T243A}}$ induced similar levels of the glutamatergic selector gene $t / x 3$ (Fig. 3.4F). To identify if the more wild-type like activity of Ptf1 $1 a^{\mathrm{T} 243 \mathrm{E}}$ is only the result of its stronger neuronal differentiation promoting activity compared to Ptf $1 \mathrm{a}^{\mathrm{T} 243 \mathrm{~A}}$, gad1a and $t / \times 3$ activation by those two constructs was investigated after normalization to tubb2b expression. Although the expression levels of gad1a were very similar after tubb2b normalization, expression of other markers for a GABAergic lineage such as prdm13 (Fig3.4B; Table S1) were stronger activated by $\operatorname{Ptf} 1 \mathrm{a}^{\mathrm{T} 243 \mathrm{E}}$ than by $\mathrm{Ptf} 1 \mathrm{a}^{\mathrm{T} 243 \mathrm{~A}}$, while the glutamatergic markers $t / \times 3$ and $s / c 17 a 7$ induction was stronger by Ptf1a ${ }^{\mathrm{T} 243 \mathrm{~A}}$ (Fig. 3.4B; Table S1). Altogether these results indicate that although Ptf1a ${ }^{\mathrm{T} 243 \mathrm{E}}-\mathrm{GR}$ is still inducing a mixed neuronal transmitter phenotype, this mutant behaved more like the wild-type Ptf1a.

\subsection{The mixed neuronal transmitter phenotype induced by Ptf1 $\mathrm{a}^{\mathrm{T} 243 \mathrm{~A}}$ is not the result of an impaired interaction with $\mathrm{Rbpj}$}

As the interaction with Rbpj is essential for promoting a GABAergic cell fate and suppression of a glutamatergic cell fate, the mixed neuronal transmitter phenotype by Ptf1a ${ }^{T 243 A}$ might be the result of an impairment of Rbpj interaction (Hori et al., 2008; Hanotel et al., 2014). Therefore, to study the binding of Rbpj and Ptf1a in vivo, a biomolecular fluorescence complementation assay (BiFC) was performed (Fig. 3.5A). Towards this end, constructs were created in which Ptf1a, Ptf1 $\mathrm{a}^{\mathrm{W} 224 \mathrm{~A} / 242 \mathrm{~A}}$ or Ptf1 $\mathrm{a}^{\text {T243A }}$ were fused to the N-terminus of Venus EYFP (Ptf1a-Vn, Ptf1aW224AW242A-Vn, Ptf1 $a^{\text {T243A }}$ $\mathrm{Vn}$ ) as well as a construct in which the C-terminal fragment of Venus EYFP was fused to Rbpj. As a positive control, the C-terminal fragment of Venus EYFP was fused to the ubiquitously expressed class $A$ bHLH protein E12. For

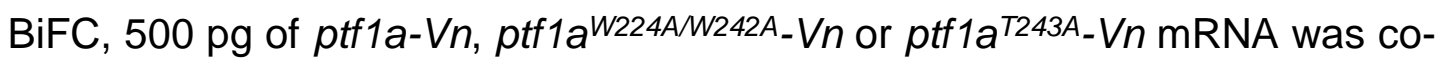


injected together with $500 \mathrm{pg} r b p j-V_{c}$ or e12-Vc mRNA animally in both blastomeres of two-cell stage $X$. laevis embryos. To identify injected cells, 100 pg mRFP mRNA was also co-injected. At blastula stage, the embryos were dissociated to single cells and imaged (Fig. 3.5B).

All three Ptf1a-Vn constructs, when co-injected with E12-Vc, showed a strong nuclear BIFC signal, demonstrating their universal ability to form heterodimers with E12 (Fig. 3.5C and D). A nuclear BiFC signal could be detected in $49 \%$ of RFP positive cells expressing Rbpj-Vc together with Ptf1a-Vn but not with Ptf1aW224AW242A-Vn (Fig. 3.5 C, D). This recapitulates previous results indicating the importance of these two tryptophan residues in the C-terminus of Ptf1a for the interaction with Rbpj and demonstrates the functionality of the BiFC assay (Beres et al., 2006; Hori et al., 2008). In contrast to

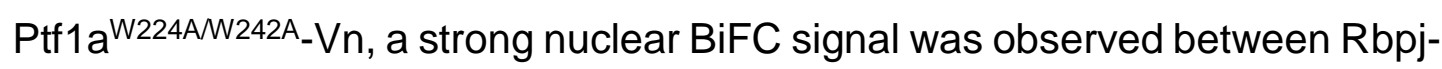
Vc and Ptf1 $\mathrm{a}^{\mathrm{T} 243 \mathrm{~A}}-\mathrm{Vn}$ (Fig. 3.5C and D), demonstrating that the mutation in the threonine does not significantly alter binding to Rbpj.

Altogether, those data show that the induced mixed neuronal transmitter phenotype by Ptf1 $\mathrm{a}^{\mathrm{T} 243 \mathrm{~A}}$ is not the result of an impaired interaction with Rbpj, suggesting that another mechanism is responsible for the induced neuronal transmitter phenotype.

\subsection{Ptf1aT 243 mutants strongly bind to Prdm13}

Ptf1a suppressed a glutamatergic cell fate through the direct activation of Prdm13, which in turn inhibits the expression of the glutamatergic selector gene t/x3 (Chang et al., 2013; Borromeo et al., 2014; Hanotel et al., 2014). Furthermore, it has been shown that Prdm13 directly binds to the $t / x 3$ regulatory regions and suppress $t / x 3$ expression in mouse in a mechanism that involves direct binding to the DNA by Prdm13, as well as recruitment to the promoter of $t / x 3$ by the bHLH transcription factor Ascl1 (Chang et al., 2013). Interestingly, overexpression of Ptf1 $\mathrm{a}^{\mathrm{T} 243 \mathrm{~A}}$ in $X$. laevis ectodermal explants induces prdm13 expression as well as the expression of $t / x 3$, which would somehow contradict the previous studies demonstrating that in the presence 
of Prdm13, t/x3 expression is inhibited. In subpopulations of prdm13expressing cells, Prdm13 represses its own activator, Ptf1a, through directly

A

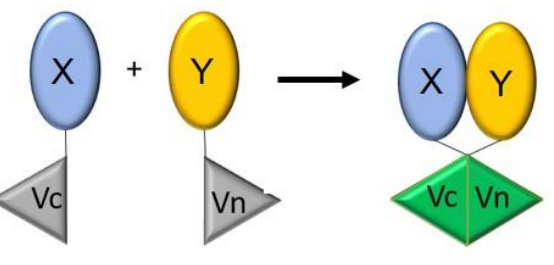

C $\quad \mathrm{E} 12-\mathrm{Vc}$
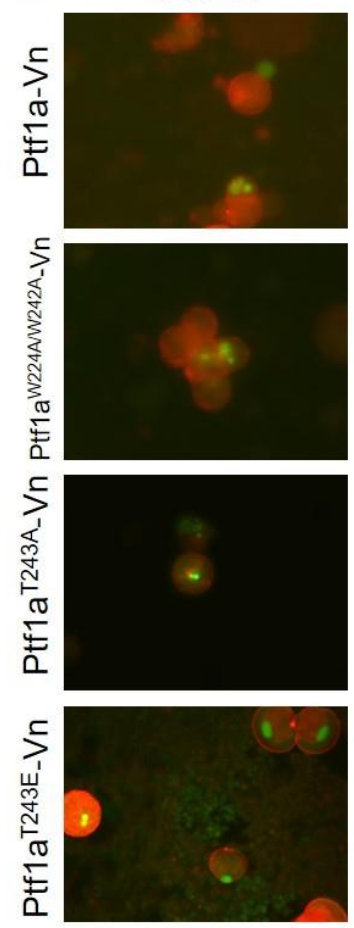

Rbpj-Vc
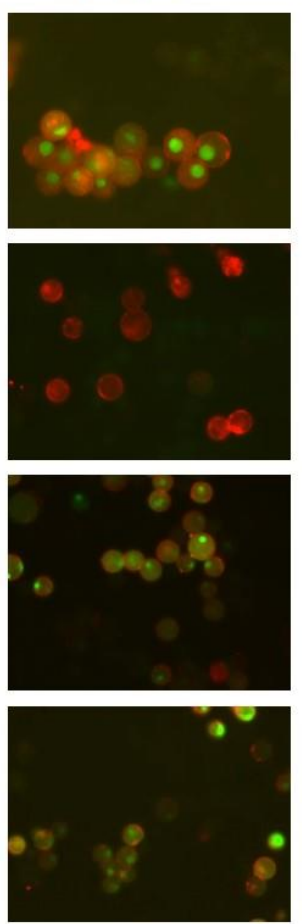

mRFP EYFP
B

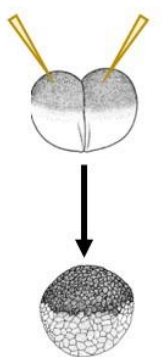

stage $8-9$

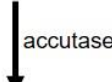

○\%

stage 10.5

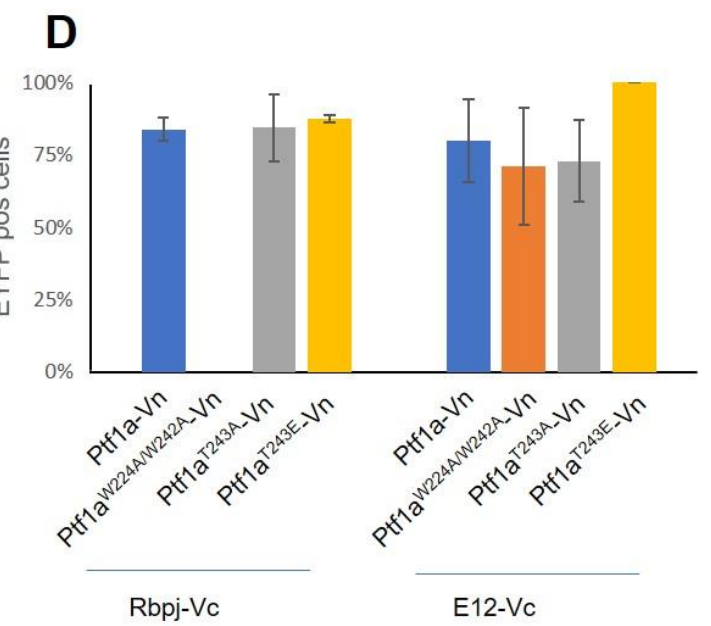

Figure 3.5: Ptf1a ${ }^{\mathrm{T} 243 \mathrm{~A}}$ mutation does not affect its ability to bind Rbpj

(A) Overview bimolecular fluorescence complementation assay (BiFC). Proteins were fused to the $\mathrm{N}$ - or $\mathrm{C}$-terminus of Venus-EYFP. Interaction of proteins of $\mathrm{X}$ and $\mathrm{Y}$ will lead to the formation of a complete EYFP, which can be detected by its fluorescence. (B) Experimental procedure: $500 \mathrm{pg}$ of ptf1a-Vn, ptf1a ${ }^{T 243 E}-V n$, $p t f 1 a^{W 224 A}$ W242A $-V n$ or ptf1 $a^{T 243 A}-V n$ mRNA were co-injected with 500 pg of either rbpj$V c$ or e12-Vc mRNA and with $100 \mathrm{pg}$ of $m R F P$ mRNA in both animal blastomeres of two-cell stage. At blastula stage, cells were dissociated by the use of accutase and analyzed for fluorescence. (C) Results of the BiFC analysis. On the top, the injected construct carrying the c-terminal Venus EYFP is shown. The corresponding Nterminal Venus EYFP construct is shown on the left. Red fluorescence indicated injected cells due to the expression of mRFP, green fluorescence is the corresponding YFP signal. (D) Quantification of the BiFC analysis. The given numbers and ratios all refer to the total amount of mRFP positive cells that have been analyzed. Error bars refer to the standard error of the mean. 
binding Ptf1a and inhibiting its positive autoregulation (Bessodes et al., 2017; Mona et al., 2017). Interestingly, in the $X$. laevis retina, those cells where Prdm13 inhibits ptf1a will later gain a glycinergic instead of GABAergic cell fate (Bessodes et al., 2017). Considering the outcome of Ptf1 $\mathrm{a}^{\mathrm{T} 243 \mathrm{~A}}$ overexpression in inducing prdm13 as well as GABAergic and glutamatergic marker genes, it might be that Prdm13 needs to interact with Ptf1a to actively suppress glutamatergic markers such as $t / x 3$.

To test this hypothesis, Prdm13 was fused to the C-terminal half of Venus EYFP and BiFC was performed by co-injecting prdm13-Vc and mRFP mRNA together with ptf1a-Vn, ptf1aW224AW242A-Vn, ptf1a T243A-Vn or ptf1a T243E-Vn mRNA in both blastomeres of two-cell stage $X$. laevis embryos. At blastula stage, the embryos were dissociated and fluorescence microscopy performed to visualize BiFC (Fig. 3.6A).

Interestingly, a BiFC signal could be observed in a very low number of cells

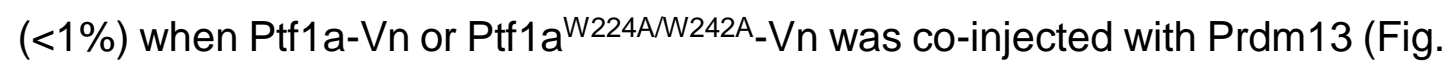
$3.6 \mathrm{~B}$ and $\mathrm{C}$ ). Surprisingly, a quite strong BiFC signal could be detected in approximately half of the Ptf1a ${ }^{\text {T243A- }} \mathrm{Vn}$ and Prdm13-Vc injected cells (Fig. 3.6B and $C$ ). Interestingly, in some of the cells showing EYFP fluorescence, the BiFC signal was not restricted to the nucleus, but additionally scattered in several aggregations throughout the cytoplasm (Fig. 3.6D). Surprisingly, a strong BiFC signal could be detected when Ptf1 $\mathrm{a}^{\mathrm{T} 243 \mathrm{E}}-\mathrm{Vn}$ was co-expressed with Prdm13-Vc (Fig. 3.7B). Here too, aggregates of EYFP signal could be detected in several cells. Quantification showed that the number of cells showing interaction with Prdm13 resembled those of Ptf1 $\mathrm{a}^{\mathrm{T} 243 \mathrm{~A}}-\mathrm{Vn}$ (Fig. 3.6C). Altogether, the data show that the Ptf1 $\mathrm{a}^{\mathrm{T} 243}$ play an essential role in the neuronal cell fate inducing activity and mutations in it, lead to a strong interaction of Prdm13, but do not alter its association with Rbpj or E12. 
A

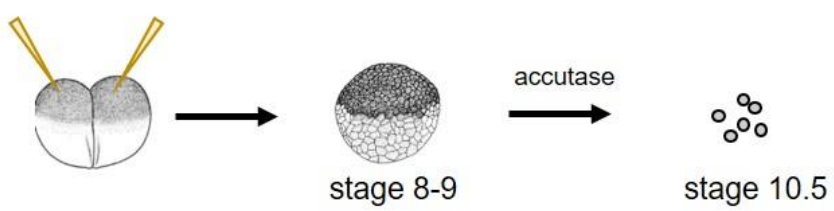

B Prdm13-Vc
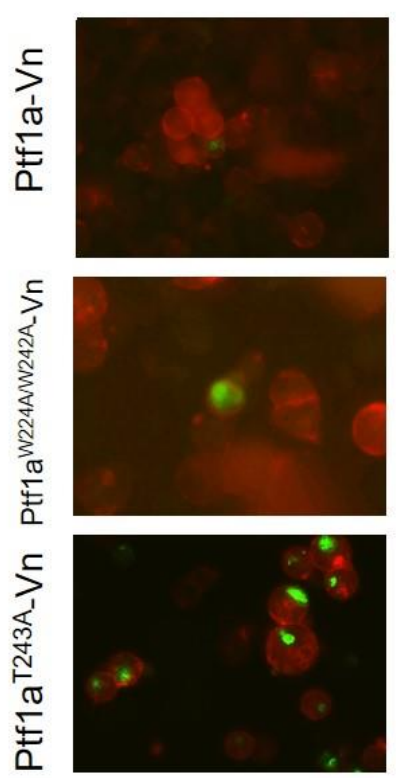

담

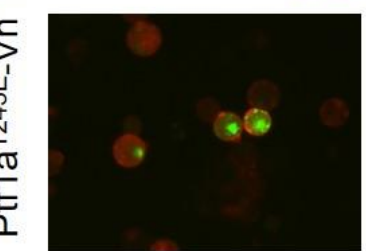

mRFP EYFP
C

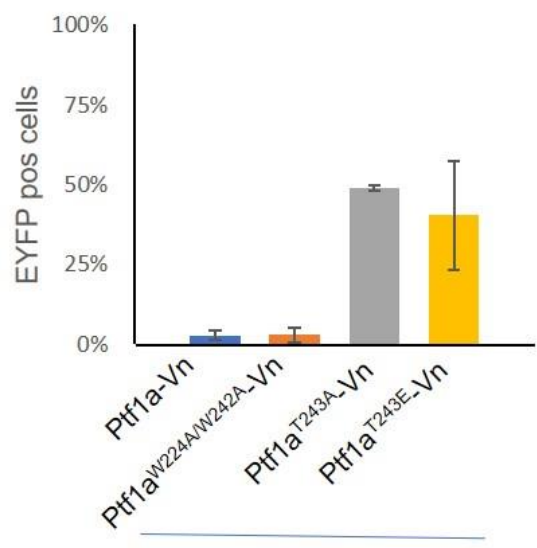

Prdm13-Vc

D mRFP

EYFP
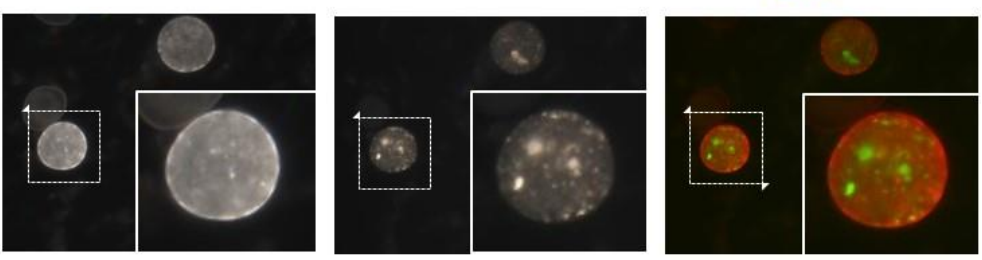

Figure 3.6: Ptf1a ${ }^{T 243 A}$ and Ptf1a ${ }^{\text {T243E }}$ mutants strongly interact with Prdm13.

(A) Experimental procedure: 500 pg of ptf1a-Vn, ptf1a $a^{\text {T243E }}-V n$, ptf1a $a^{\text {W224A } 2424 A}-V n$ or ptf1 ${ }^{T 243 A}-V n$ mRNA were co-injected with 500 pg of prdm13-Vc mRNA and with 100 pg of mRFP mRNA in animally both blastomeres of two-cell stage embryos. At blastula stage, cells were dissociated by the use of accutase and analyzed for fluorescence. (B) BiFC analysis. Indicated on the top are the injected constructs carrying the C-terminal Venus-EYFP. The corresponding $\mathrm{N}$-terminal Venus-EYFP construct is shown left. Injected cells are stained red due to their expression of mRFP, while green is the corresponding EYFP signal indicating BiFC. C) Quantification of the BiFC analysis. The given numbers and ratios all refer to the total amount of mRFP positive cells that have been analyzed. Error bars refer to the standard error of the mean. D) Example for a "scattered" distribution of the EYFP signal within a cell. Given are the channels for mRFP (left) and EYFP (middle) alone and merged (right). 


\subsection{Ptf1a induces direct target genes at different time points}

For a better understanding of the mechanisms driving the development to a distinct neuronal subtype induced by Ptf1a, it is important to understand which genes were induced by Ptf1a. Previous studies investigating the transcriptional program induced by Ptf1a after 6 and $25 \mathrm{~h}$ in $X$. laevis ectodermal explants, afforded insight into the temporal control of Ptf1a gene induction (Hedderich, 2012). In another analysis, Ptf1a was overexpressed in animal caps in the presence of the translational inhibitor cycloheximide $(\mathrm{CHX})$ to identify direct target genes of Ptf1a. Through comparison of both analysis, it could be shown that several Ptf1a direct target genes were not activated in an immediate early fashion, but were delayed in their activation (Hedderich, 2012). Direct target genes showing an immediate activation by Ptf1a consist mostly of genes involved in neuronal differentiation such as the Notch ligand delta1 (dll1) or the neuronal differentiation genes ebf2 or myt1, as well as prdm13. Among the delayed direct target genes were genes like gad1a, barhl2, kirrel2 or neurog2.

To get a better understanding in the mechanics for the delayed target gene induction, the exact onset of Ptf1a target gene induction needs to be defined. Therefore, $20 \mathrm{pg}$ of ptf1a-GR mRNA was injected into the animal hemispheres of both blastomeres at the two-cell stage. At blastula stage, the animal cap was excised and protein activity was induced for 3, 6, 9, 12, 15 and $25 \mathrm{~h}$. The RNA was isolated and analyzed by quantitative Nanostring analysis using a codeset consisting of known early and late Ptf1a target genes and neuronal marker genes (Fig. 3.7A, Table S7).

To ensure that constructs indeed behaved as expected, the late time point at the $25 \mathrm{~h}$ was first analyzed for known Ptf1a-downstream genes. Compared to the non-injected control explants, Ptf1a-injected animal caps strongly expressed the pan-neuronal marker tubb2b, the direct target genes of Ptf1a, prdm13 and kirrel2 as well as the GABAergic marker gad1a, slc32a1 and pax2, but not the glutamatergic selector gene $t / x 3$ and the vesicular glutamate transporter s/c17a7 (Fig. 3.7B, Table S3). This expression reflects the activities of Ptf1a in promoting neuronal differentiation, inducing a GABAergic cell fate and inhibiting a glutamatergic cell fate (Glasgow et al., 2005; Hoshino et al., 
2005; Dullin et al., 2007; Pascual et al., 2007). (Fig. 3.7B). The Ptf1a-induced genes analyzed can be classified into groups based on their timing of activation (Fig. 3.7 C-H, Table S3). The first group of genes has the onset of their activation already at $3 \mathrm{~h}$ (dll1, ebf2, myt1, prdm13, prdm14, zc3h12c), the second at $6 \mathrm{~h}$ (barhl2, kirrel2, Ihx1, nr5a2, onecut1.2, $t / \times 3$, aldh1b1), the third group between 9 and $12 \mathrm{~h}$, with a strong tendency towards $12 \mathrm{~h}$ (pax2, tubb2b, gad1a, neurog2, pdia2) and one gene at $25 \mathrm{~h}$ (slc32a2). Under those genes activated at the earliest stages $(3 \mathrm{~h}, 6 \mathrm{~h})$ were direct Ptf1a target genes (prdm13, kirrel2) (Nishida et al., 2010; Chang et al., 2013) as well as many genes predicted to be direct Ptf1a target genes by their activation in presence of the translational inhibitor cycloheximide (CHX) (Schneider-Poetsch et al., 2010) could be found (dll1, ebf2, myt1, zc3h12c) (Hedderich, 2012), but known and predicted direct target genes were also present under those genes activated at the $12 \mathrm{~h}$ time point (neurog2, gad1a, pdia2) (Henke et al., 2009; Hedderich, 2012) (Fig. 3.7C-H, Table S4). Interestingly, the activation of delayed Ptf1a target genes followed the activation of the post-mitotic neuronal marker tubb2b, which indicates that the expression of those genes may be connected with cell cycle exit.

Interestingly, a significant induction of $t / x 3$ expression by Ptf1a could be detected in early stages, which is increasing up to the $9 \mathrm{~h}$ time point, but afterwards is declines and is no longer detected after $25 \mathrm{~h}$ (Fig. 3.7H), indicating that during the onset of neuronal differentiation Ptf1a induces $t / x 3$ expression, which is subsequently suppressed during subtype specification. Altogether the data suggest that the delayed activation of Ptf1a direct target genes begins their onset of expression $12 \mathrm{~h}$ following Ptf1a induction. As only a limited number of genes were analyzed, it is unclear if all the delayed target genes behave in such a manner. 
A
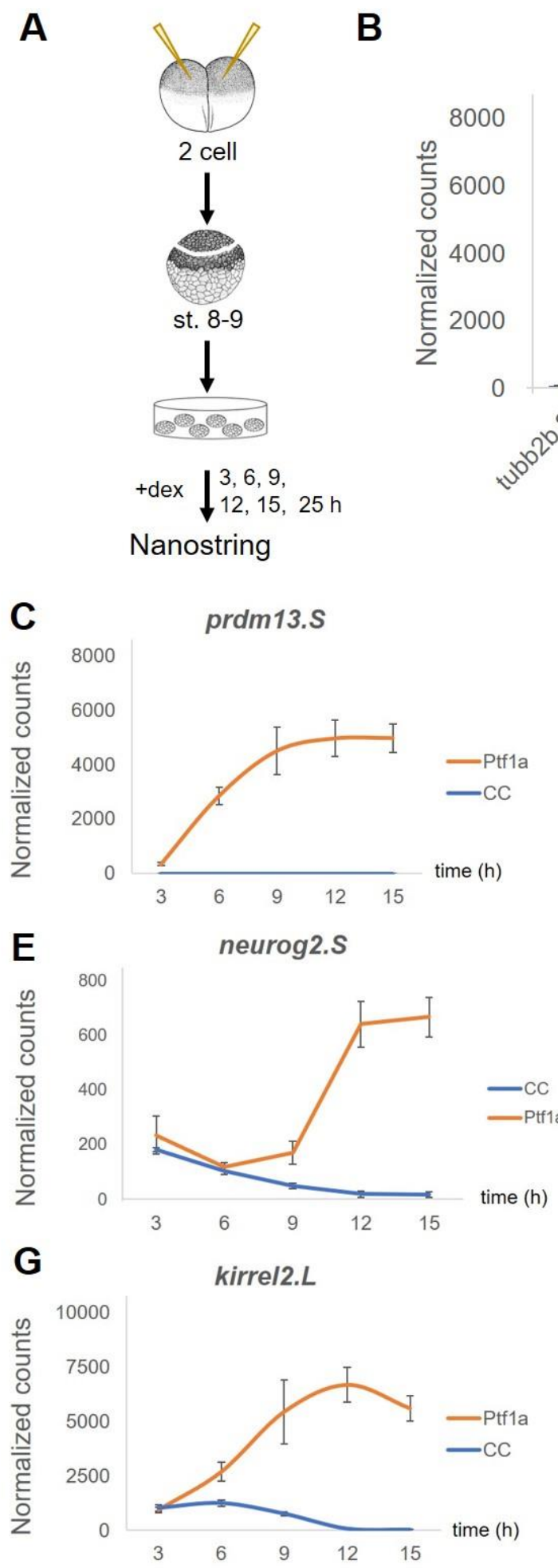

\section{Expression at $25 \mathrm{~h}$}

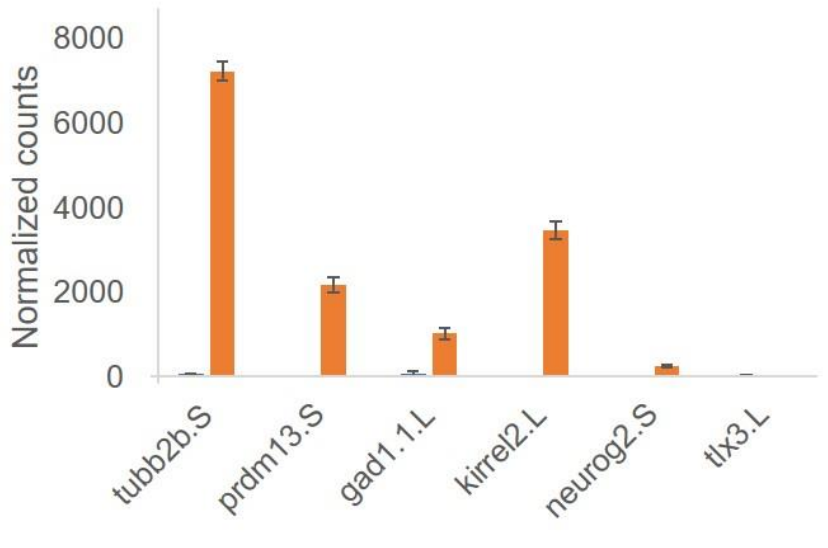

-CC - Ptfla
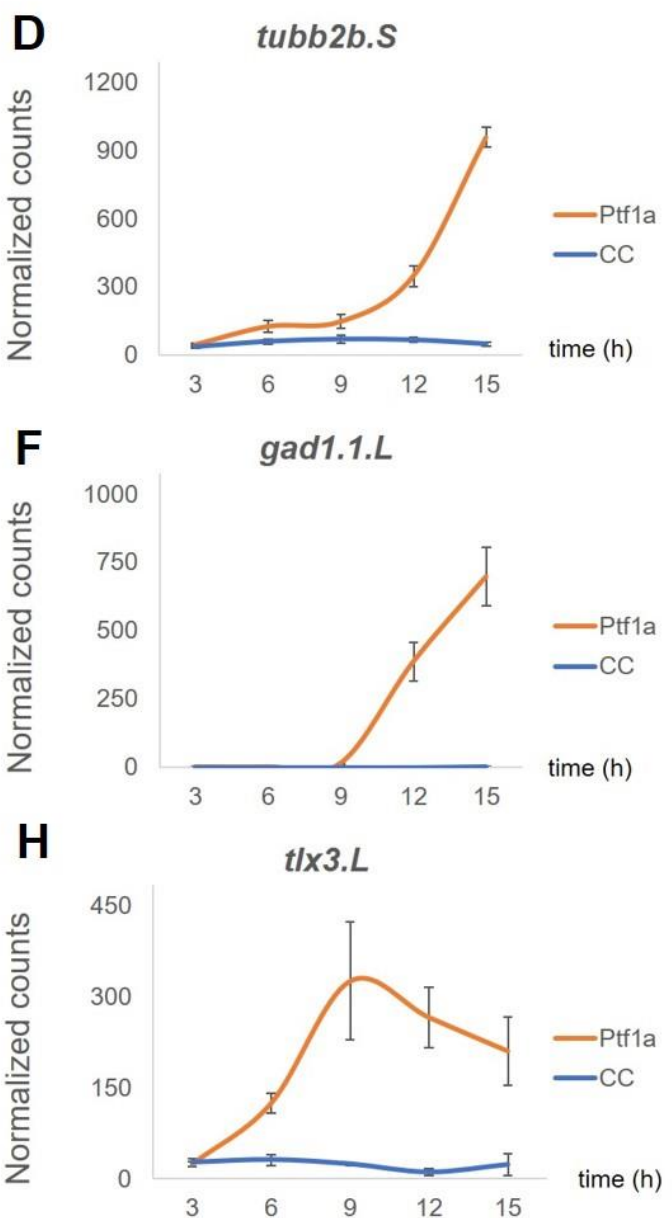

Figure 3.7: Ptf1a is activating its target genes in a temporal manner

(A) Experimental procedure: $20 \mathrm{pg}$ of ptf1a-GR, mRNA was injected animally in both blastomeres of two-cell stage embryos. At blastula stage, animal caps were excised. Protein activity was induced by dexamethasone treatment for 3, 6, 9, 12, 15 and 25 h. Animal caps were collected for RNA isolation at the indicated times and gene expression quantified by Nanostring analysis. (B) Results of the quantitative Nanostring analysis. Shown are the average expression levels of marker genes from three biological replicates. Error bars refer to the standard error of the mean. Data $\rightarrow$ 
were normalized for the expression levels of the housekeeping gene ppi1l. $(\mathrm{C}-\mathrm{H})$ Results of the quantitative Nanostring analysis. Shown are the average expression levels of marker genes from three biological replicates. Error bars refer to the standard error of the mean. Data were normalized for the three housekeeping gene ppi1l. .S and.$L$ refer to the corresponding $X$. laevis homeolog targeted by the Nanostring probe. Note the different scales in each diagram. CC, control cap.

\subsection{Temporal expression of Ptf1a target genes by RNA sequencing}

To understand the temporal induction of Ptf1a target genes on a global level, RNA sequencing was performed by injecting $20 \mathrm{pg}$ of ptf1a-GR mRNA animally in both blastomeres of the two-cell stage blastomeres. At blastula stage, animal caps were excised and protein activity was induced by the addition of dexamethasone to the culture medium. After 3,6 or $12 \mathrm{~h}$, the RNA was isolated and analyzed by RNA-seq (Fig. 3.8A).

To get an insight in the similarity between the different replicates, the distinct samples were clustered according to their similarity using the R/Bioconductor environment (www.bioconductor.org). Analysis clearly showed a high similarity in the $3 \mathrm{~h}$ and $6 \mathrm{~h}$ groups, which much higher similarity than any of these groups to the $12 \mathrm{~h}$ time point samples, supporting the argumentation that a major change at the $12 \mathrm{~h}$ time point has occurred (Fig. 3.8B)

Applying a cutoff of at least a $\log _{2}$ fold-change of two over non-injected control caps, 133 genes were upregulated by Ptf1 a after $3 \mathrm{~h}$ of protein induction, while after $6 \mathrm{~h}$ and $12 \mathrm{~h}$ of protein induction, 241 and 468 genes were upregulated (Fig. 3.8B). In contrast, only 15 genes were downregulated after $3 \mathrm{~h}$ of protein induction, while after $6 \mathrm{~h}$ and $12 \mathrm{~h}, 3$ and 20 genes were downregulated. From these, 140 and 353 genes also had their onset of induction after $6 \mathrm{~h}$ and $12 \mathrm{~h}$. Under the genes being upregulated, known Ptf1a target genes like prdm13 (Hedderich, 2012; Chang et al., 2013) or kirrel2 (Nishida et al., 2010) could be found (Table S14-16). 
A

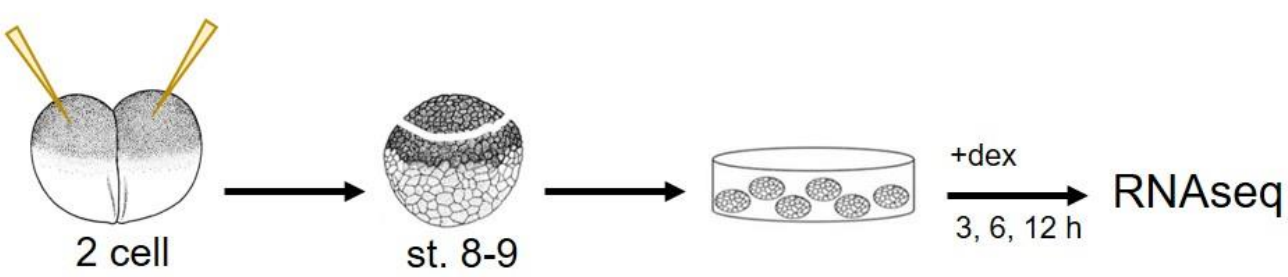

B

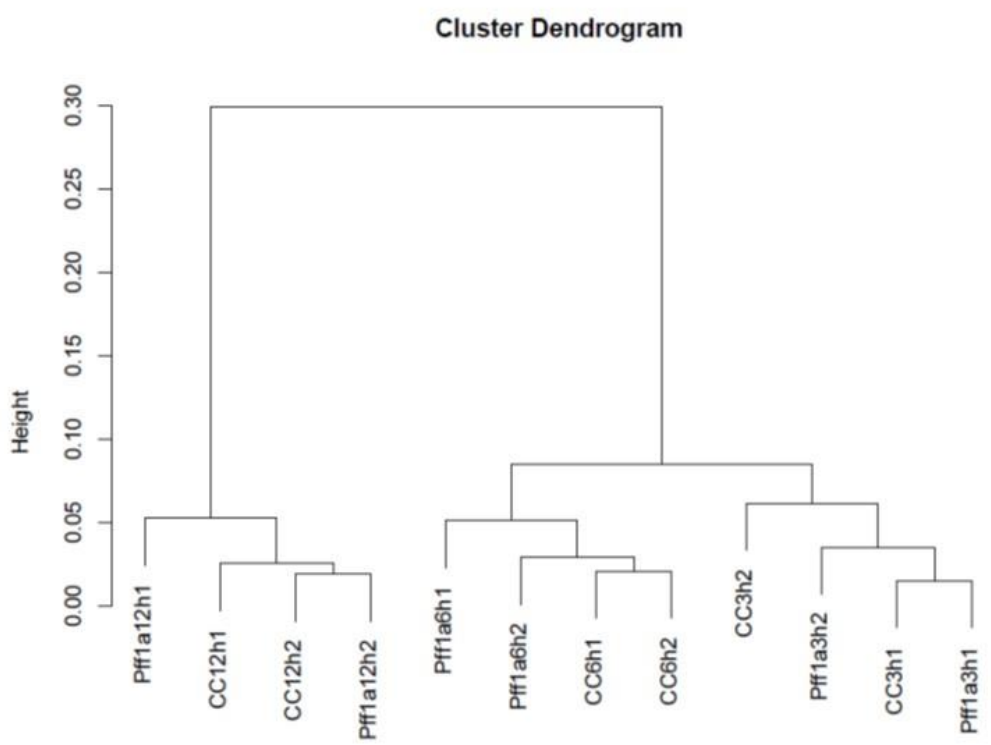

Figure 3.8: Summary of the RNAseq

(A) Experimental procedure: $20 \mathrm{pg}$ of ptf1a-GR, mRNA was injected in both animal blastomeres of two-cell stage. At blastula stage, animal cap is excised. Protein activity is induced by dexamethasone treatment for 3, 6, and $12 \mathrm{~h}$. Afterwards, the animal caps were collected for RNA isolation. RNA expression was analyzed by RNAseq. (B) Cluster Dendrogram showing the similarity of the different samples.

To identify the direct target genes being upregulated at the distinct time points, the list of upregulated genes from the RNA-seq was compared to a list of genes induced by Ptf1a in the presence of the translational inhibitor cycloheximide (CHX) (Hedderich, 2012). From the 133 genes upregulated at least four-fold after 3 h, 59 genes could be identified as direct target genes (Fig. 3.9C). Of the genes upregulated after $6 \mathrm{~h}, 90$ were direct target genes. From those, $41 \%$ (37 genes) had their onset at $6 \mathrm{~h}$. After $12 \mathrm{~h}, 99$ direct target genes could be found. Interestingly, approximately half of the direct target genes (45 genes) activated after $12 \mathrm{~h}$ of protein induction had their onset at this time point, indicating a greater change in the activated target genes occurring at this time point 
A

$$
\log _{2} \mathrm{FC} \geq 2
$$

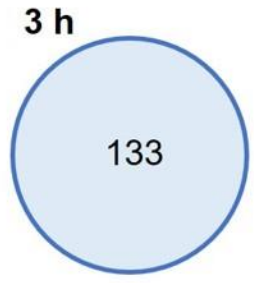

B

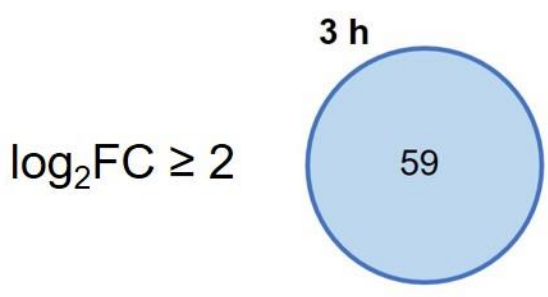

$6 \mathrm{~h}$

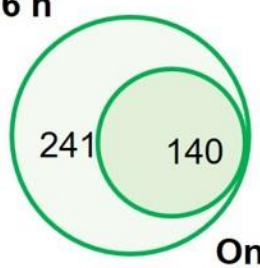

Onset $6 \mathrm{~h}$

$6 \mathrm{~h}$

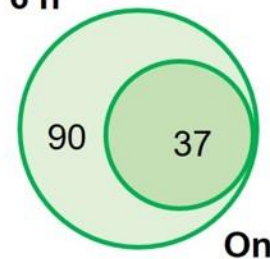

$12 \mathrm{~h}$

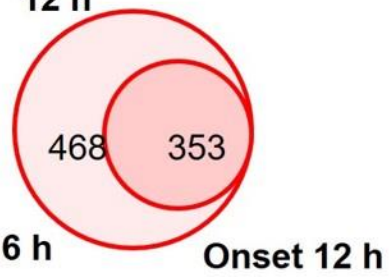

Onset $12 \mathrm{~h}$

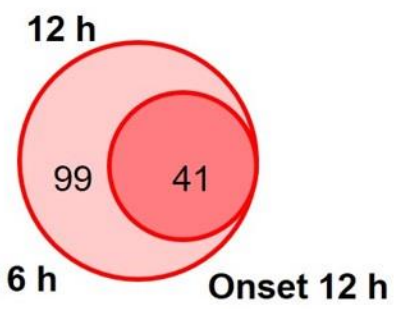

Figure 3.9 Comparison of target gene activation by Ptf1a after 3, 6 and $12 \mathrm{~h}$

Venn diagrams indicating number of upregulated genes at 3,6 and $12 \mathrm{~h}$ by the given threshold in general $(A)$ and for direct target genes only $(B)$. Given is the number of genes with a $\log _{2}$ fold change higher or equal than 2 compared to non-injected control caps.

\subsection{Ptf1a target genes are highly enriched for playing roles during transcription and neurogenesis}

To identify the function of the upregulated genes at the different time points, gene ontology analysis was performed using DAVID (Database for Annotation, Visualization and Integrated Discovery, http://david,abcc.ncifcrf.gov/). GO analysis revealed an enrichment of the genes upregulated after $3 \mathrm{~h}$ involved in the positive or negative regulation of transcription (e.g. myt1, dll1, ebf2, prdm13), the development of the nervous system (e.g. dll1, olig2, Ihx1) or neuron fate commitment (e.g. notch1, id2, olig2) (Fig. 3.10A). Gene ontology analysis for the genes upregulated after $12 \mathrm{~h}$ of protein induction also showed a significant enrichment in processes for positive or negative regulation of transcription (barlh2, Ihx1, neurog1, neuro2, myt1, olig2) and the development of the nervous system (Ibx1, olig2, barlh2, myT1, Ihx1, neurog1, neurog2, neurod4) (Fig. 3.10B). At both time points, a high overlap in the genes listed in the different GO terms could be detected, as for example dll1, olig2 or myt1 
were involved in transcriptional regulation as well as in the development of the neural system after $3 \mathrm{~h}$. Similarly, neurog1, neurog2, myt1 or barlh2 were involved in transcriptional regulation as well as in the development of the neural system after $12 \mathrm{~h}$. Nevertheless, no strong differences between the gene function between the 3 and $12 \mathrm{~h}$ time point could be detected.

A $3 h$

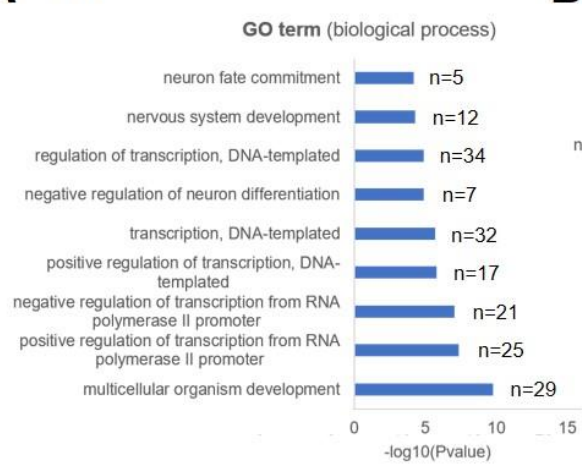

C onset $3 \mathrm{~h}$ direct

$$
\text { GO term (biological process) }
$$

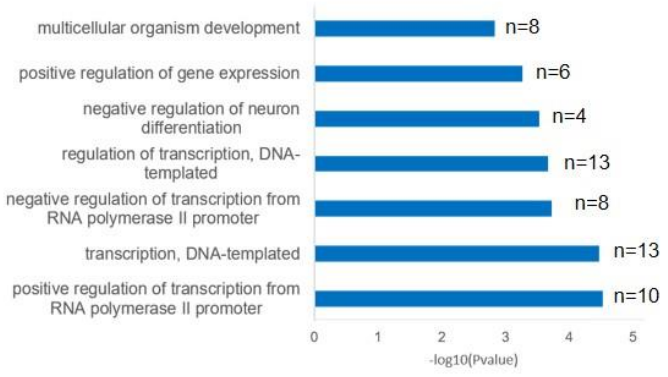

B $12 \mathrm{~h}$

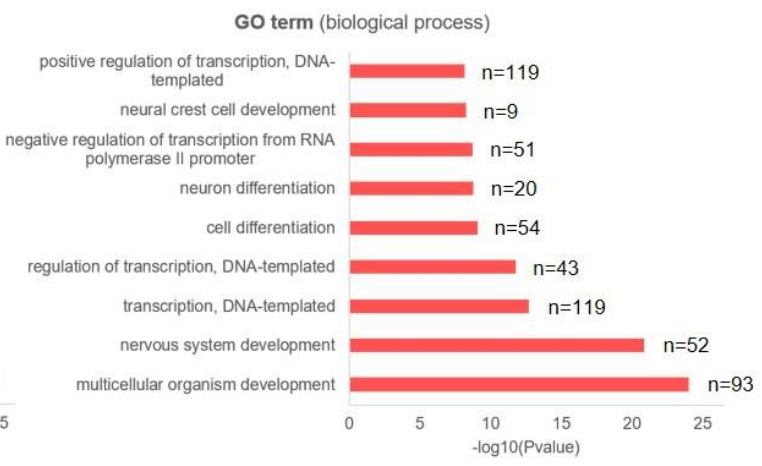

D onset $12 \mathrm{~h}$ direct

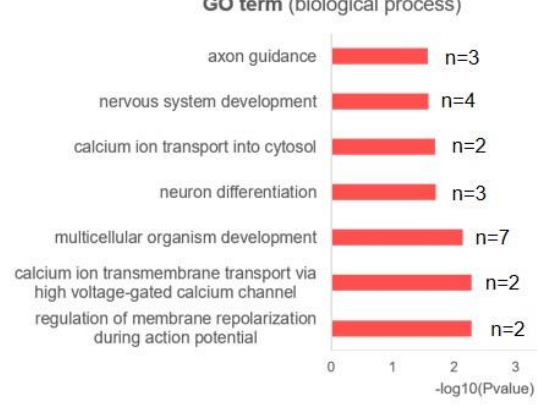

Figure 3.10: Genes involved in processes involved in neurogenesis are enriched in all three time points

Gene Ontology (GO) analysis was performed using DAVID (http://david.abcc.nclfcrf.gov) (Dennis et al. 2003). The number of genes in each category is shown as well as the -log10 P-value of false discovery rate. Shown are the top listed terms of each category ranked by their $\mathrm{P}$-value. The different diagrams refer to genes activated after $3 \mathrm{~h}$ (blue) and $12 \mathrm{~h}$ (red) in general $(\mathrm{A})$ and for direct target genes having their onset at the given time point (B)

When considering only those direct target genes that were upregulated at the particular time point, however, differences could be found (Fig. 3.10C and D). While direct target genes induced after $3 \mathrm{~h}$ still were strongest enriched for positive or negative regulation of transcription (e.g. ebf2, myt1, prdm13 or dll1) (Fig. 3.10C), direct target genes having their onset of expression after $12 \mathrm{~h}$, 
were involved in axon guidance (e.g. neurog2, tubb3) regulation of membrane repolarization during action potential or calcium transport (cacna2d1, cacnb3), or neuron differentiation (neurog2, tubb3, unc5a) (Fig. 3.10D) indicating that genes expressed after $12 \mathrm{~h}$ play a role in function and maturation of neurons, while early induced genes are more likely involved early neuronal differentiation.

\subsection{A knock-down of Brg1 affects the induction of indirect Ptf1a target genes}

Chromatin remodeling mediated by the BAF chromatin remodeling complex plays an essential role during vertebrate neurogenesis (Bachmann et al., 2016). It had been shown that a knock-down of Brg1, one of the two catalytic subunits of the BAF complex lead to an impairment in the induction of neuronal differentiation by proneuronal transcription factors like Neurog2 (Seo et al., 2007). As induction of delayed Ptf1a target genes was connected with the induction of the post-mitotic neuronal marker tubb2b, the delayed activation of direct target genes might be dependent on chromatin remodeling via the BAF chromatin remodeling complex. To investigate which genes are affected by a Brg1 knock-down in Ptf1a overexpressing animal caps, RNA sequencing was performed using stage 14 ectodermal explants from embryos injected with 20 pg of ptf1a-GR mRNA together with $20 \mathrm{ng}$ of either control morpholino (cMO) or Brg1 morpholino (Brg1-MO) previously shown to be effective in knockdown of Brg1 by inhibiting translation (Seo et al., 2007) (Fig 3.11A).

In total, Ptf1a together with the control MO upregulated more than 819 genes by applying a threshold of $\log _{2}$ fold-change of two compared to non-injected control caps and an FDR value $<0.05$, while 668 genes were upregulated by Ptf1a in the presence of the Brg1-MO. From these upregulated genes, in total, 527 genes were shared by both samples, while 236 genes were exclusively induced by Ptf1a-GR and the cMO (Fig3.11B) 
A

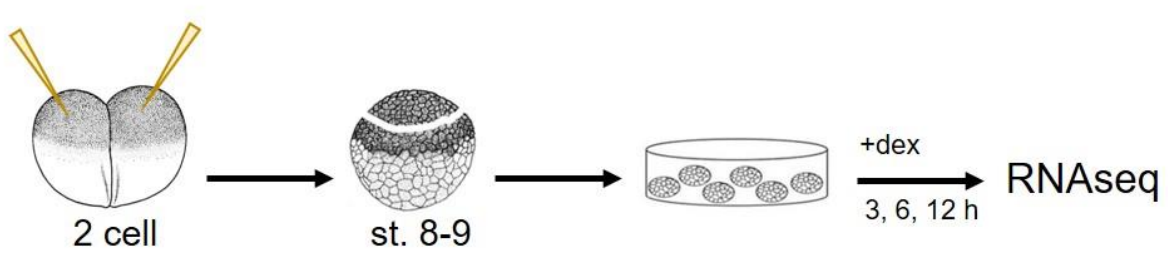

B

$\log _{2} \mathrm{FC} \geq 2$

C $\log _{2} \mathrm{FC} \geq 1$

Ptf1a+ Brg1MO

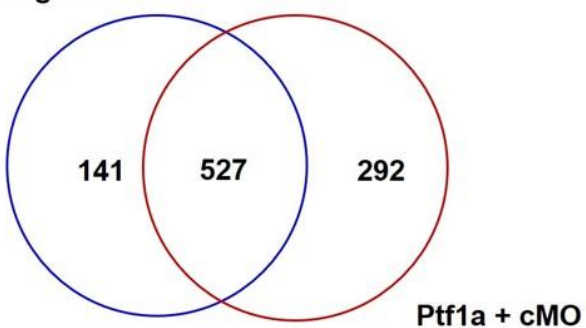

Brg1 affected

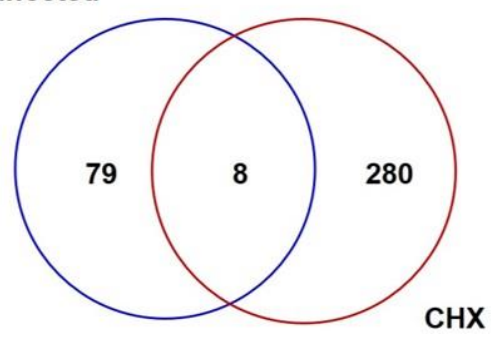

Figure 3.11: Brg1 dependent genes are mostly indirect Ptf1a target genes

(A) Experimental procedure: $20 \mathrm{pg}$ of ptf1a-GR mRNA was injected individually or together with either a control morpholino or a Brg1 morpholino in both animal blastomeres of two-cell stage. At blastula stage, animal cap is excised. Protein activity was induced by dexamethasone treatment. At stage 14, the animal caps were collected for RNA isolation. RNA expression was analyzed by RNA sequencing. (B) Venn Diagram showing the number of unique and shared genes being at least fourfold (left) or 16-fold upregulated by Ptf1a in presence of cMO (blue) or Brg1-MO (red) (C) Venn Diagram showing the number of unique and shared genes being at least four-fold (left) or two-fold (right) upregulated by Ptf1a and cMO over Ptf1a together with the Brg1-MO (blue) and by a list of genes induced by Ptf1a in the presence of CHX (Hedderich, 2012) (red).

To identify, which genes were most strongly affected by a Brg1 knock-down, the gene expression levels were compared between Ptf1a in the presence of the Brg1-MO versus the presence of the control MO. In the presence of the Brg1-MO, 56 genes were upregulated and one of these genes were induced by Ptf1a in presence of $\mathrm{CHX}$ (Hedderich, 2012) and 87 genes were significantly reduced by more than two-fold (Fig. 3.11C). As only a limited number of genes were altered, the influence of the Brg1 knock-down on target gene induction by Ptf1a is specific for selected genes. Tubb2b could be found under those genes showing a strong impairment in Ptf1a induction upon Brg1 knock-down. From the 87 genes negatively affected by the Brg1-MO, only eight genes (flnc.S, nr5a2.S, tubb3.S, six3.L, traf4.S, LOC100496628-like.S, emilin1.L, st18.L) were identified as direct target genes by Ptf1 a based on their ability to activated in the presence of $\mathrm{CHX}$ (Hedderich, 2012). (Fig. 3.11C) 


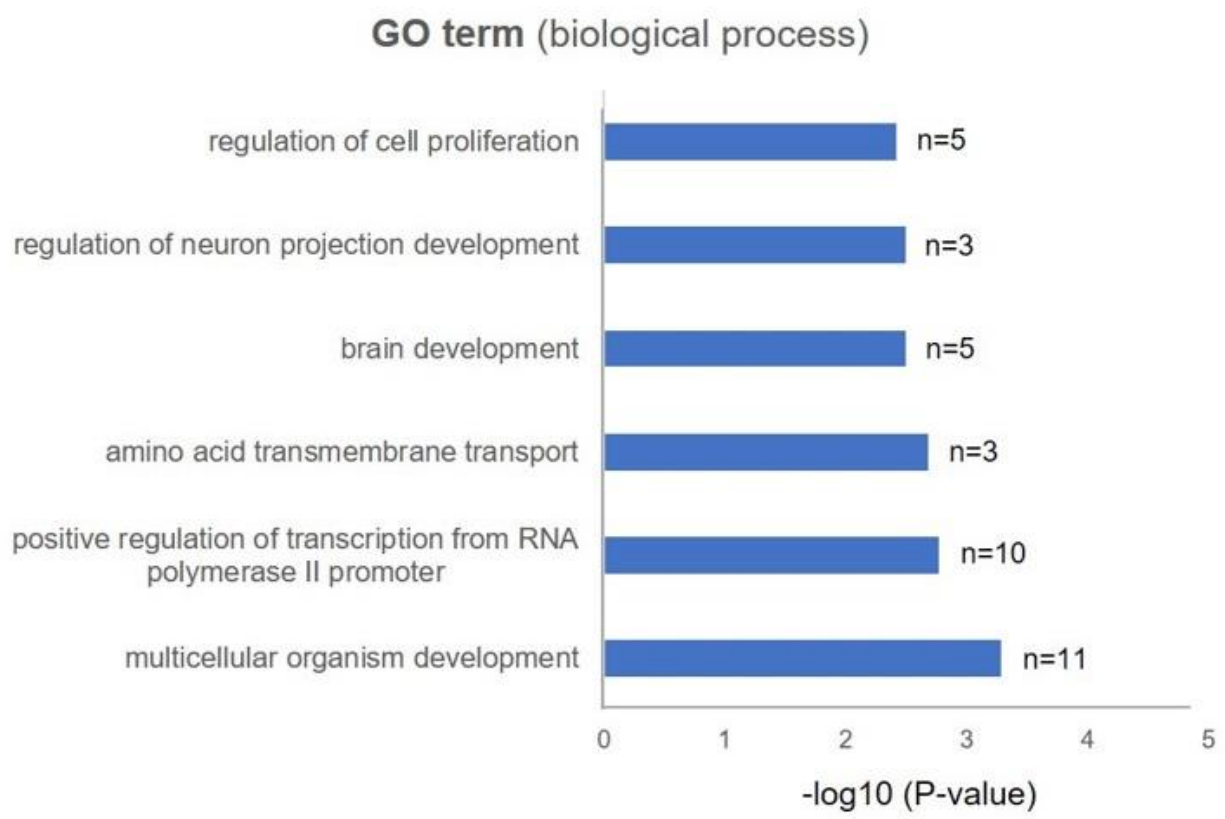

Figure 3.12: Genes affected by an Brg1 knock-down have activities during neurogenesis

$\mathrm{GO}$ analysis of genes being at least four-fold downregulated in the presence of the Ptf1a together with Brg1-MO versus the control MO. Analysis was performed using DAVID (http://david.abcc.nclfcrf.gov) (Dennis et al., 2003). Shown are the top listed terms of each category ranked by their P-value. The number of genes in each category is shown to the right of the bar diagram.

For identification, which gene functions were affected by a Brg1 knockdown, Gene Ontology analysis was performed on the strongest Brg1-MO affected genes using DAVID. Corresponding to the Ptf1a gene function, genes affected by a Brg1 knock-down were enriched in terms having a function during neurogenesis, including "brain development" (nes, bcr, six3), or regulation of neuron projection development" (pak3, sfrp2), but also "regulation of cell proliferation" (ppp1r9b, nr5a2) (Fig. 3.12).

Altogether, these data suggest that most Ptf1a direct target genes do not require chromatin remodeling by the Brg1 containing BAF complex.

\subsection{Induction of selected Ptf1a downstream genes requires Brg1}

For verification of the RNA sequencing results, $20 \mathrm{pg}$ of ptf1a-GR mRNA was injected alone or in combination with $20 \mathrm{ng}$ of a control $\mathrm{MO}$ or the Brg1-MO, in 
A

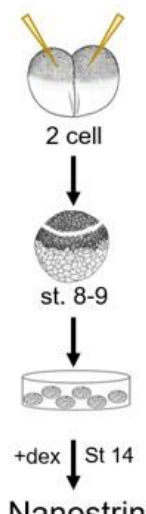

Nanostring
B

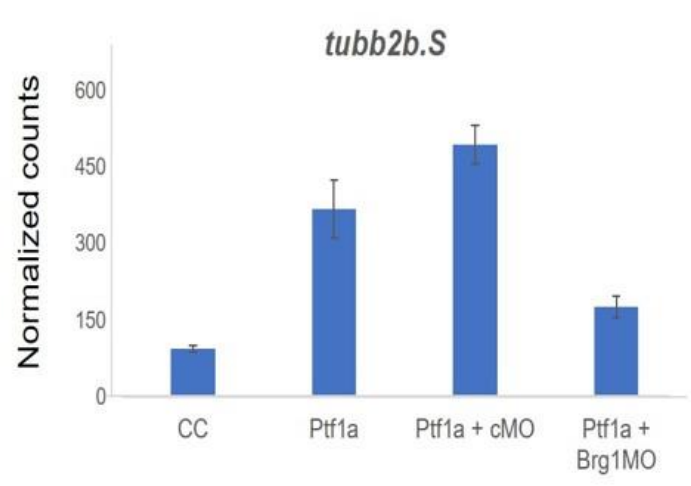

D

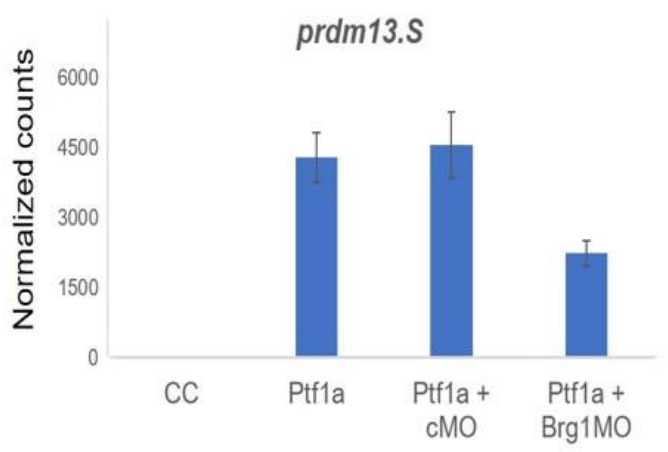

$\mathbf{F}$

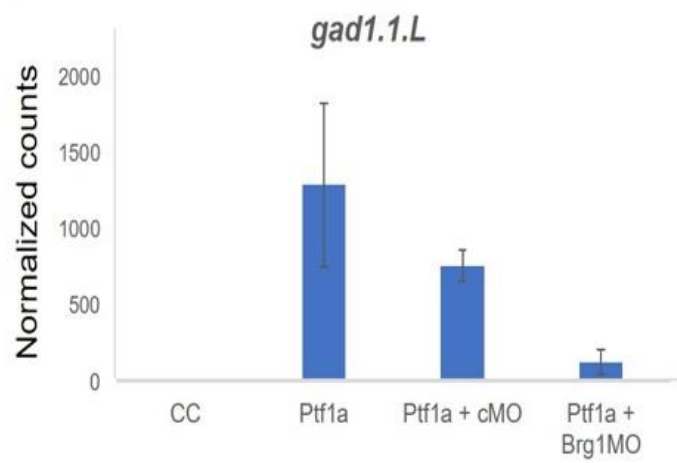

Figure 3.13: Induction of some delayed Ptf1a target genes is depended on Brg1

(A) Experimental procedure: $20 \mathrm{pg}$ of ptf1a-GR mRNA was injected individually or together with either a control morpholino (cMO) or a Brg1 morpholino (Brg1MO) in both animal blastomeres of two-cell stage. At blastula stage, animal cap is excised. Protein activity was induced by dexamethasone treatment. At stage 14, the animal caps were collected for RNA isolation. RNA expression was analyzed by quantitative Nanostring analysis. B-F) Examples of Brg1 dependent or independent target genes. Shown are the average expression levels of marker genes from three biological replicates. Error bars refer to the standard error of the mean. Data were normalized for the three housekeeping genes odc, ppi1/ and rp/p0. Note the different scales in each diagram. 
both blastomeres at the two-cell stage. At blastula stage, animal caps were excised and protein activity was induced by the addition of dexamethasone to the culture medium. At the equivalent of stage 14, the RNA of the explants was isolated and gene expression was analyzed by quantitative Nanostring analysis using a codeset containing Ptf1a direct and indirect early and late target genes, as well as selected markers of neuronal differentiation and subtype specification (in total 96 genes were analyzed) (Fig. 3.13A, Table S7).

Overexpression of Ptf1a upregulated almost all investigated target genes previously shown to be upregulated by Ptf1a in the RNA-seq (Fig. 3.14, Fig. 3.15). As it was previously shown for other proneural transcription factors like Neurog2 (Seo et al., 2007) and the Ptf1a-Brg1-MO RNAseq analysis, the

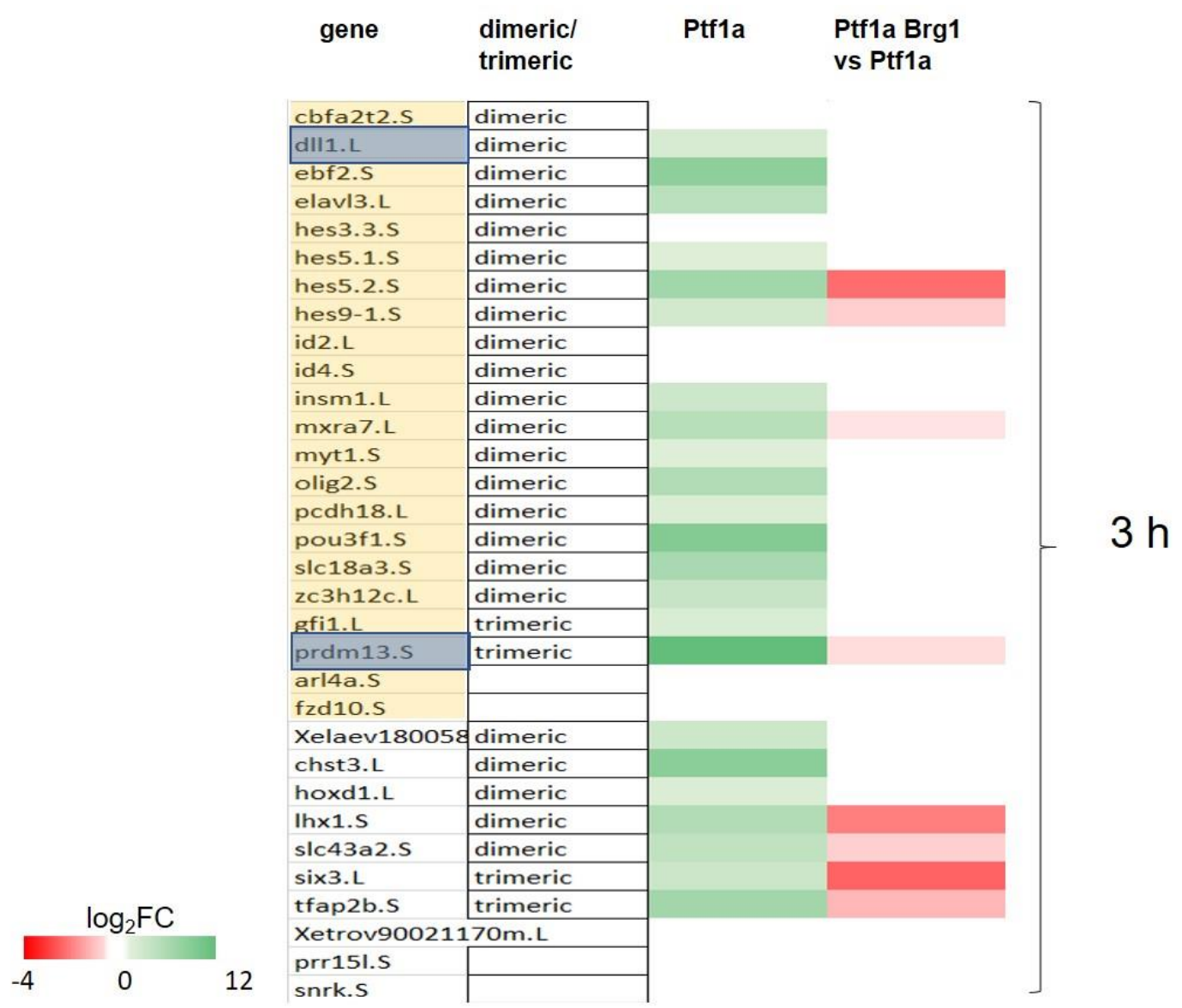

Figure 3.14 Summary of the Nanostring analysis for genes induced after $3 \mathrm{~h}$

Shown is a heatmap showing the log 2 fold-change activation of target genes by Ptf1a (green) or the fold-change reduction when Co-injected with Brg1 morpholino compared to the Co-injection with a control morpholino (red). Genes activated by Ptf1a in presence of $\mathrm{CHX}$ are highlighted in yellow. 
upregulation of $t u b b 2 b$ expression by Ptf1a was significantly attenuated in the presence of the Brg1-MO compared to the control MO (Fig. 3.13B), indicating the importance of Brg1 for neuronal differentiation. Ptf1a direct target genes such as the early upregulated dll1 or the late upregulated neurog2, were not affected by a Brg1 knockdown (Fig. 3.13C, E). Interestingly, with respect to the timing of target gene induction, most of the observed genes activated within 3 $\mathrm{h}$ of Ptf1a induction were not affected by a Brg1 knock-down. From those early induced target genes being affected by a Brg1 knock-down, four were direct target genes (out of 22 direct Ptf1a target genes present for this time point), while four were indirect Ptf1a target genes (out of 10 indirect Ptf1a target genes present for this time point). In general, in the target genes analyzed, the number of indirect target genes affected by the Brg1 knock-down was higher than the number of direct target genes (Fig. 3.17G). For later activated genes, including both direct (e.g. neurod4 and gad1a) and indirect (e.g. pou3f2 or Ibx1), the number of Brg1 dependent genes increases. All genes being affected the Brg1 knock-down in the RNA-seq that were analyzed, were also affected in the Nanostring analysis (Fig. 3.17G). Furthermore, the finding from the RNA-seq that many affected genes were indirect Ptf1a target genes could be demonstrated in the Nanostring analysis. However, many genes like gad1a or prdm13 that showed no significant reduction in the RNA-seq analysis, showed a reduction in the induction by Ptf1a in the presence of the Brg1-MO (Fig. 3.13D, Fig. 3.14) Altogether, the data suggest that the induction of most early target genes by Ptf1a is not dependent of chromatin remodeling via the BAF chromatin remodeling complex, while this mechanism becomes more important for the induction of selected late target genes. However, BAFmediated chromatin remodeling cannot be the only mechanism responsible for the delayed target gene activation as even at the $12 \mathrm{~h}$ time point, induction of several direct Ptf1a target genes is not affected by a Brg1 knock-down. 


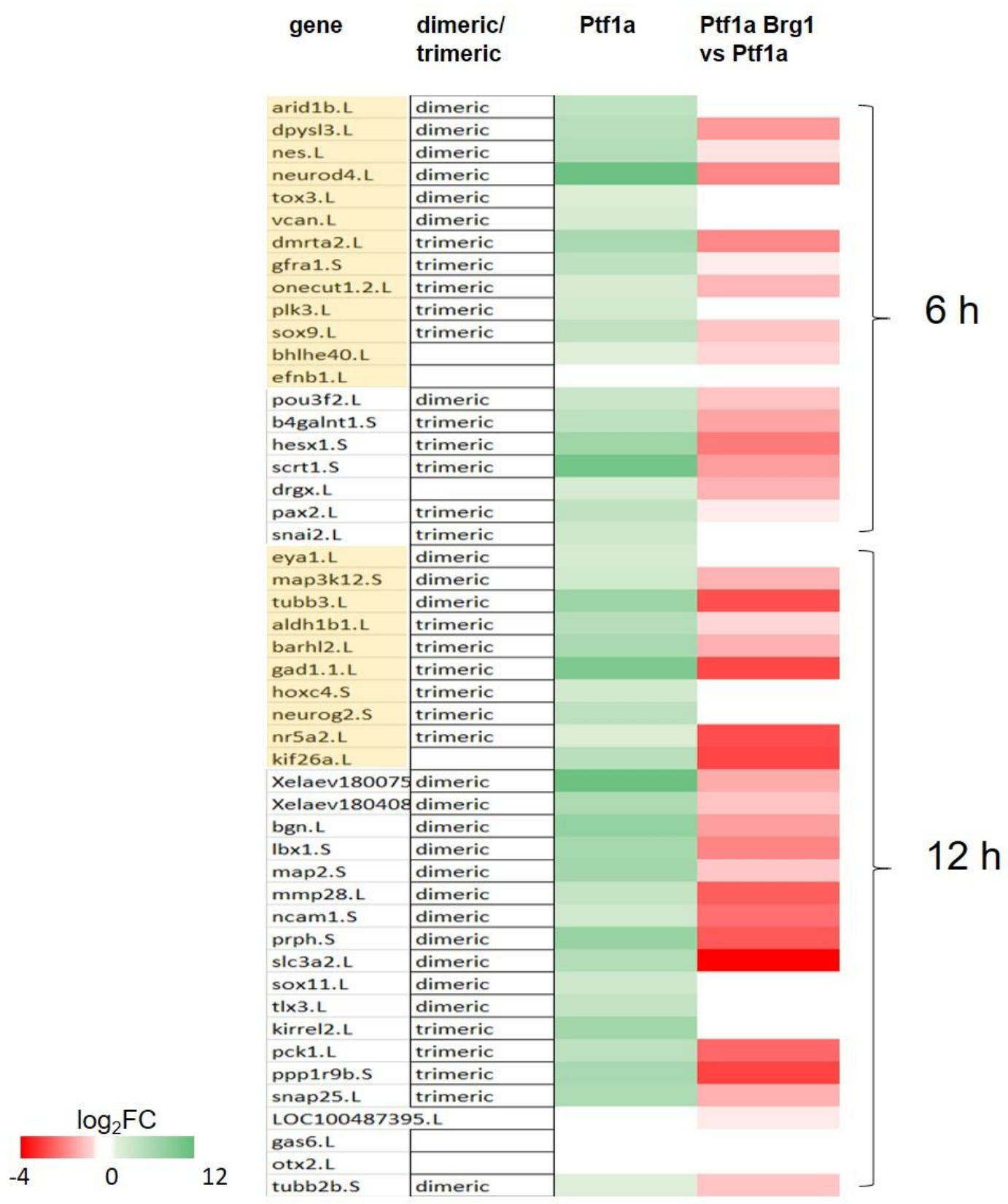

Figure 3.15 Summary of the Nanostring analysis for genes induced after $\mathbf{3 ~ h}$

Shown is a heatmap showing the log2 fold-change activation of target genes by Ptf1a (green) or the fold-change reduction when Co-injected with Brg1 morpholino compared to the Co-injection with a control morpholino (red). Genes activated by Ptf1a in presence of $\mathrm{CHX}$ are highlighted in yellow.

\subsection{Ptf1a can alter the chromatin state of its target genes}

Since other chromatin remodeling complexes exist that are Brg1 independent it cannot be ruled out that that the delayed activation of Ptf1a is due to chromatin accessibility. Therefore, analysis of the chromatin landscape of 
Ptf1a direct target genes were analyzed by using ATAC-seq (Assay for Transposase Accessible Chromatin). Therefore, $20 \mathrm{pg}$ of ptf1a-GR was injected in both blastomeres at the two-cell stage of $X$. laevis embryos. At blastula stage, the animal caps were excised and protein activity induced by dexamethasone treatment for 3 (when the earliest target genes are activated) or $12 \mathrm{~h}$ (the timing when late target genes were activated). Afterwards, ATAC sequencing was performed (Fig. 3.16A, B). The results of the ATAC sequencing were compared to the expression levels of the genes obtained from the RNA-seq to determine the correlation between the chromatin levels with the target gene expression.

In total between $100,000,000$ and $200,000,000$ reads could be mapped for each sample. However, many duplicate reads could be found in the samples, so that after removal of those duplicates only between 2,000,000 and $12,000,000$ reads remained, so that the amounts of reads that remained was quite low indicating restriction in the analysis due to a non-sufficient quality of the samples.

Nevertheless, genes strongly expressed in the naïve animal cap ( $3 \mathrm{~h}$, equivalent stage 10 or $12 \mathrm{~h}$, equivalent stage 12.5) like odc (data not shown), pou5f3.2 or fcgbp could be located in areas of quite open chromatin in the presence and absence of Ptf1a (Fig 3.16C). Time dependent changes in the loci of genes could be detected, with pou5f3.2 at early stages, for example, was located in a quite open area. At later stages, however, the chromatin at the pou5f3.2 locus becomes much more closed, which correlated with a decrease in pou5f3.2 expression levels (Fig. 3.16C, D). The opposite effect could be identified for example on the expression of $f c g b p$. Here, the fcgbp gene locus is located in closed chromatin regions at the $3 \mathrm{~h}$ time point. At the $12 \mathrm{~h}$ time point, however, the chromatin at the fcgbp locus is quite accessible, matching with a strong increase of fcgbp expression levels (Fig. 3.16E, F). These results highlight the correlation between chromatin remodeling and gene expression and demonstrate the validity of the analysis. 
A

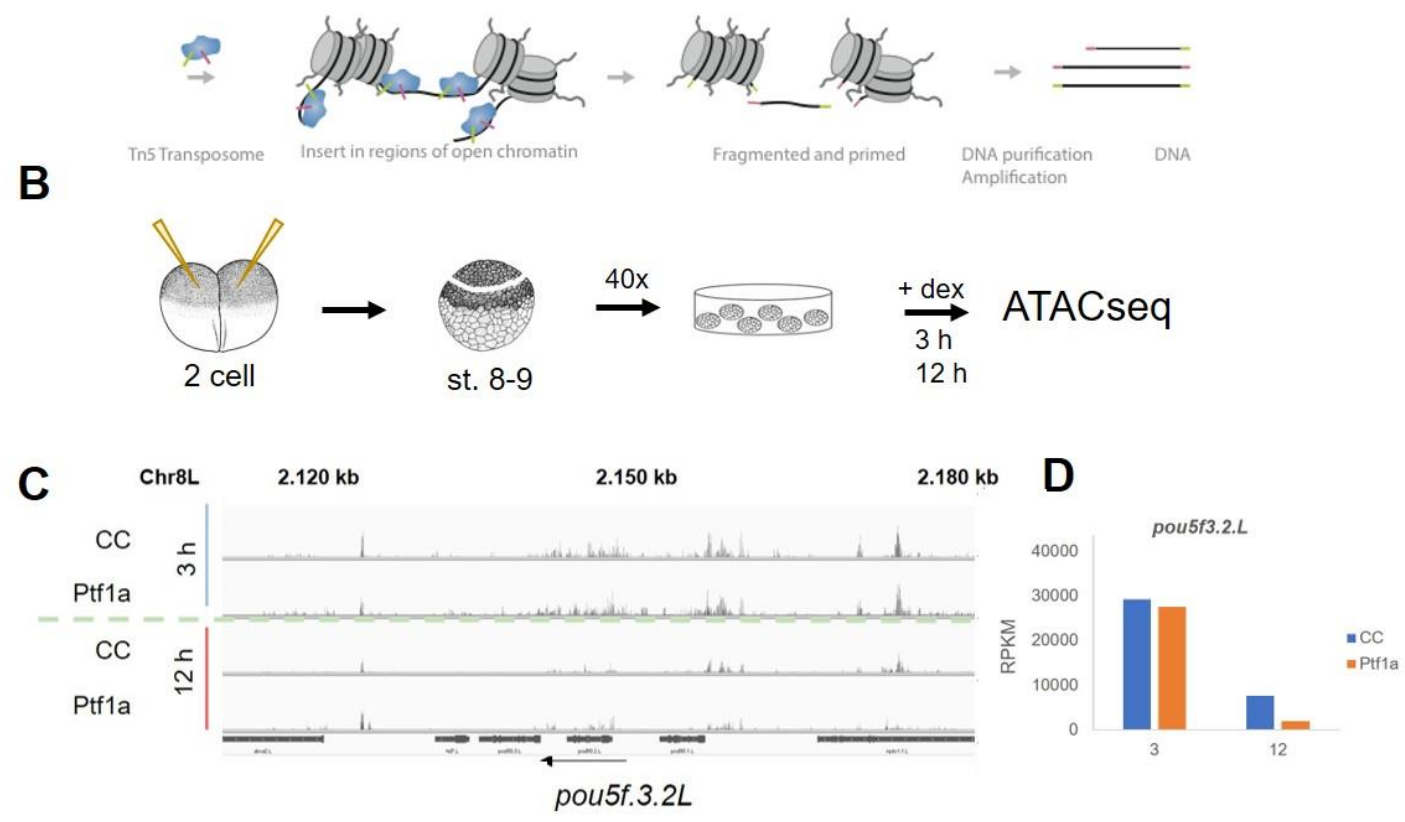

$\mathbf{E}$

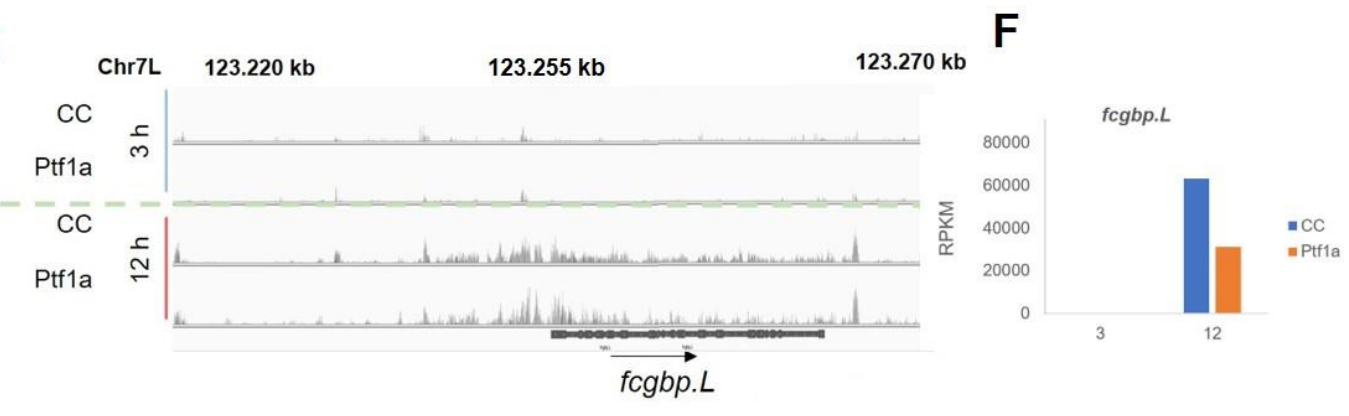

G

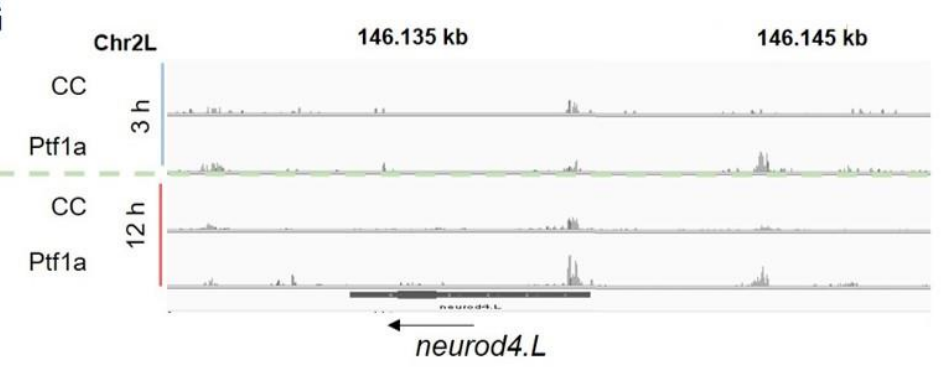

H

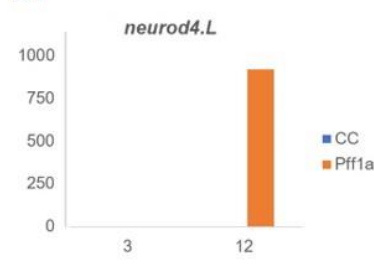

Figure 3.16: Ptf1a overexpression can change the chromatin state of its target genes

(A) Overview ATAC-seq (Scheme modified from (Buenrostro et al., 2013)). Intact nuclei were treated with a highly reactive Tn5 transposase. The transposase binds at accessible chromatin regions introducing signaling sequences. Accessible regions were amplified via PCR against the introduced sequences. PCR library is then sequenced with $50 \mathrm{bp}$ paired-end reads. (B) Experimental procedure: $20 \mathrm{pg}$ of ptf1a$G R$, mRNA was injected in both animal blastomeres of two-cell stage. At blastula stage, animal cap is excised. Protein activity is induced by dexamethasone treatment for 3 and $12 \mathrm{~h}$. Afterwards, the animal caps were collected for ATAC sequencing. (C, $\mathrm{E}, \mathrm{G})$ ATAC tracks from read coverage files mapped to the $X$. laevis genome. The corresponding sample control Cap (CC) or Ptf1a injected (Ptf1a) and the $\rightarrow$ 
corresponding time point is given on the left. The transcriptional direction of the investigated gene is indicated with a black arrow. The grey bars represent the total numbers of reads at the corresponding position of the genome. (D, G, H) The corresponding target gene's expression level from the RNAseq is shown on the right side. Note the different scales.

When looking at the loci of Ptf1a direct target genes (in total 44 genes, from those, 6 genes were activated after 3 h, 10 after $6 \mathrm{~h}$ and 24 genes after $12 \mathrm{~h}$ ). Ptf1a dependent changes in the chromatin states could be found. At the locus of the Ptf1a direct target gene neurod4, for example, a region of open chromatin could be detected approximately $5 \mathrm{~kb}$ upstream of the transcription start site in the Ptf1a injected explants, but not in non-injected control explants (Fig. 3.16G). Neurod4 expression levels, however, could not be detected at the $3 \mathrm{~h}$ time point, even though the open chromatin region appeared at this time point in the Ptf1a expressing explants (Fig 3.16H). However, in the RNAseq analysis expression of neurod 4 becomes detectable at the $6 \mathrm{~h}$ time point (Table S15), indicating that even though the chromatin at this position is accessible, some time is needed until transcription can start. Together, these results indicate that overexpression of Ptf1a can lead to changes in the chromatin state of selected genes target genes.

\subsection{Ptf1a target genes lay in closed chromatin regions}

To identify if chromatin remodeling is responsible for the delayed activation of target genes, the chromatin state of Ptf1a target gene loci have been investigated and compared with their onset of expression. Interestingly most of the investigated early and late target genes were located in regions of rather closed chromatin, which was even the case for early induced genes like prdm13 (Fig 3.17A). 


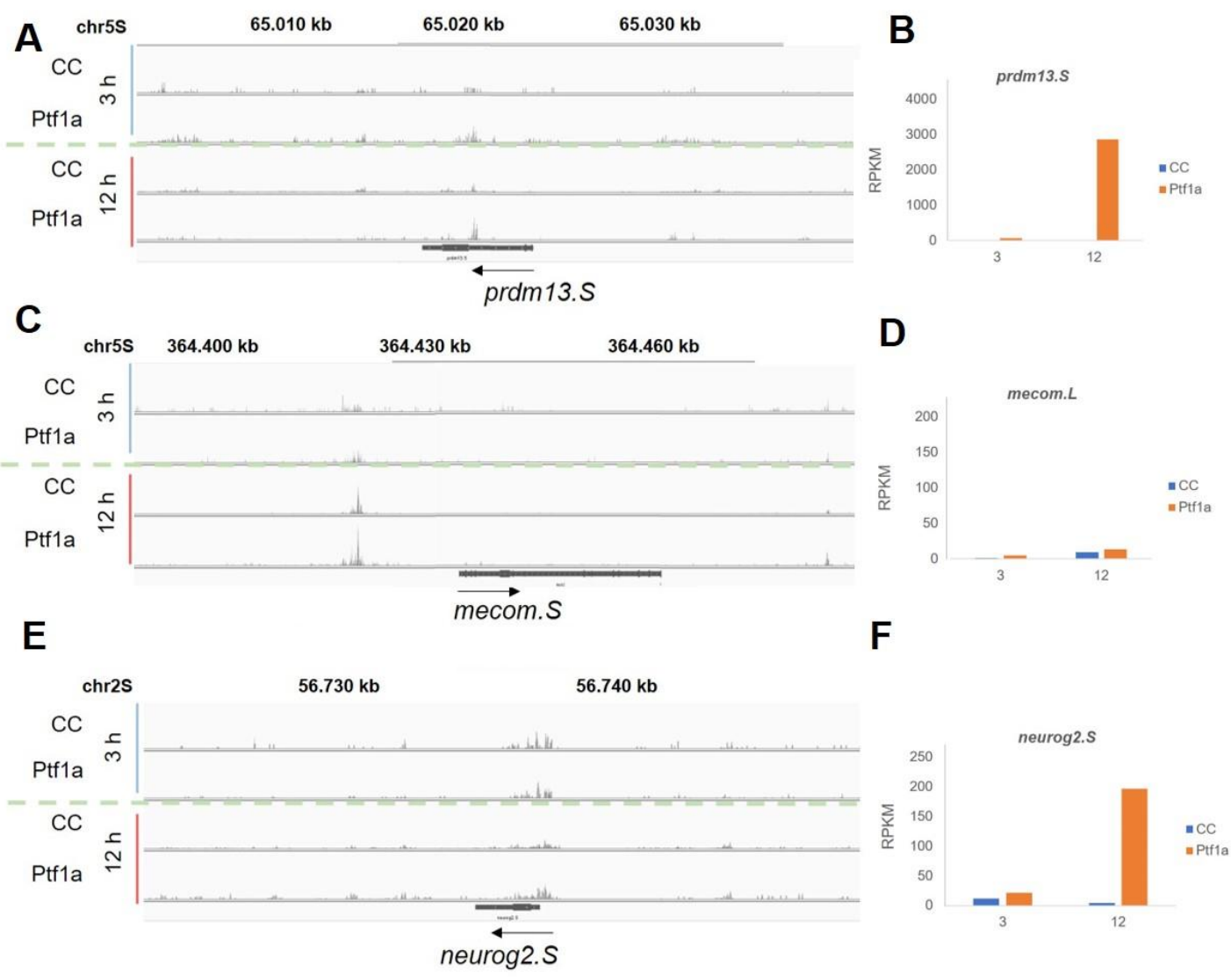

Figure 3.17: Chromatin remodeling plays a minor role in the delayed induction of Ptf1a target genes

(A, C, E) ATAC tracks from read coverage files mapped to the $X$. laevis genome. The corresponding sample (control Cap (CC) or Ptf1a injected (Ptf1a) and the corresponding time point is given on the left. The transcriptional direction of the investigated gene is indicated with a black arrow. The grey bars represent the total numbers of reads at the corresponding position of the genome. $(B, D, F)$ The corresponding target gene's expression level from the RNAseq is shown on the right side. Note the different scales.

Even though the transcriptional start site was accessible for most genes, no significant changes in chromatin accessibility at the loci of those direct target genes could be found at the time points analyzed (Fig. 3.17A, C, E). This finding was the case for time dependent changes, as well as for Ptf1a dependent changes in the chromatin accessibility. Even late induced target genes, such as mecom (Fig 3.17E) or neurog2 (Fig 3.17C) showed no significant differences in their chromatin levels over time or a change due to Ptf1a expression, indicating that Ptf1a indeed does not need chromatin remodeling to activate the investigated direct target genes. 


\section{Discussion}

\subsection{The Ptf1a C-terminus is essential for the induced neuronal transmitter phenotype}

The bHLH transcription factor Ptf1a not only plays a critical role during neuronal subtype specification where it promotes an inhibitory GABAergic cell fate and suppresses an excitatory glutamatergic cell fate, it also possesses proneural activity (Glasgow et al., 2005; Hoshino et al., 2005; Fujitani et al., 2006; Dullin et al., 2007; Nakhai et al., 2007; Pascual et al., 2007; Hedderich, 2008; Lelievre et al., 2011; Hedderich, 2012). The neuronal subtype specificity of Ptf1a depends on its interaction with Rbpj via two highly conserved motifs in the Ptf1a C-terminus. Ptf1a mutants where the Rbpj binding sites are disrupted through mutation of the tryptophan residues in the $\mathrm{C} 1$ and $\mathrm{C} 2$ domains to alanine maintain proneural activity, but the induced neurons express glutamatergic marker genes rather than GABAergic markers (Dullin et al., 2007; Hedderich, 2008; Hedderich, 2012; Hanotel et al., 2014).

In this work, a new Ptf1a mutant (Ptf1a $\left.{ }^{\mathrm{T} 243 \mathrm{~A}}\right)$ was identified, in which the threonine residue within the Ptf1a C2 domain was mutated to alanine. Overexpression of Ptf1 $\mathrm{a}^{\mathrm{T} 243 \mathrm{~A}}$ induces a mixed neuronal transmitter phenotype, by activating GABAergic markers like gad1a as well as glutamatergic markers like $t / x 3$. This mixed neuronal transmitter phenotype, however, is not due to an inhibition of Rbpj binding as demonstrated by the interaction of Rbpj and Ptf1a ${ }^{\mathrm{T} 243 \mathrm{~A}}$ through BiFC as well the upregulation of Ptf1a early (prdm13) and late (aldh1b1, barhl2, kirrel2) trimeric target genes. Albeit the induction of these genes was lower than that obtained with the wild-type Ptf1a, mutants where Rbpj interaction is impaired (Ptf1 $\left.\mathrm{a}^{\mathrm{W} 242 \mathrm{~A}}, \mathrm{Ptf} \mathrm{a}^{\mathrm{W} 224 \mathrm{~A} / \mathrm{W} 242 \mathrm{~A}}\right)$ do not interact in the $\mathrm{BiFC}$ assay and these mutants fail to induce the trimeric target genes.

Studies in mouse and $X$. laevis embryos demonstrated that overexpression of Prdm13 together with Ascl1 or Neurog2 overrides the glutamatergic promoting activity of these factors and promotes a GABAergic cell fate (Chang et al., 2013; Hanotel et al., 2014). However, overexpression of Ptf1a ${ }^{\mathrm{T} 243 \mathrm{~A}}$ in X. laevis 
animal caps results in both prdm13 and $t / x 3$ expression. Ptf1 $\mathrm{a}^{\mathrm{T} 243 \mathrm{~A}}$ is not as effective as the wild-type Ptf1a with regards to prdm13 upregulation (11-fold less), thus the amount of Prdm13 may be limiting to suppress a glutamatergic fate. However, Ptf1a $\mathrm{T}^{\mathrm{T} 243 \mathrm{E}}$ is only slightly less effective (1.5-fold less) in the induction of prdm13 compared to the wild-type Ptf1a, but still induces $t / x 3$ indicating the possibility that an unknown mechanism induced by $\mathrm{Ptf} 1 \mathrm{a}^{\mathrm{T} 243 \mathrm{~A}}$ impairs the ability of Prdm 13 to repress $t / x 3$.

Interestingly, a strong BiFC signal was observed between $\mathrm{Ptf}_{1} \mathrm{a}^{\mathrm{T} 243 \mathrm{~A}}$ and Prdm13, while only a very week signal and in few cells was observed for Ptf1a or Ptf1aW224A/W242A and Prdm13. In subpopulation of Ptf1a-expressing cells in the $X$. laevis retina that will give rise to glycinergic neurons, Prdm13 negatively regulates ptf1a (Bessodes et al., 2017). The molecular basis for Prdm13repression of ptf1a was shown in the mouse dorsal neural tube, where Prdm13 is recruited to the Ptf1a autoregulatory enhancer through direct interaction with Ptf1a (Mona et al., 2017). Thus, in the Ptf1a ${ }^{\top 243 A}$ mutant, the ability of Prdm13 to interact with Ptf1a and to suppress its activity may be increased. This would lead to a reduced induction of prdm13 by Ptf1a, so that the inhibition of $t / x 3$ is not as effective. This hypothesis is supported by the finding that $t / x 3$ is induced under $\mathrm{CHX}$ by Ptf1a (Hedderich, 2012) and that Ptf1a induced expression of t/x3 at early time points $(6-9 \mathrm{~h})$ but at later developmental stages $t / x 3$ expression is silenced.

Interestingly, overexpressing Ptf1a ${ }^{\mathrm{T} 243 \mathrm{~A}}$ in $X$. laevis embryos still affects the endogenous $t / x 3$ expression, so that only ectopically induced neurons become glutamatergic. This would indicate the presence of a second co-factor, that is induced by the wild-type Ptf1a but not by the Ptf1a ${ }^{\top 243 \mathrm{~A}}$ mutant or co-factor present in the neural tube, and needed for Prdm13 to down-regulates $t / x 3$ expression. So, it would be interesting to identify the transcription factor complex controlling the target gene induction by Ptf1a or Ptf1a ${ }^{\mathrm{T} 243 \mathrm{~A}}$ to prove this hypothesis. Furthermore, these data indicate that the induced neuronal transmitter phenotype might be the result of a mixed population of cells within the ectodermal explants, so that it would be also of interest to investigate the target gene induction by the $\mathrm{Ptf}_{1} \mathrm{a}^{\mathrm{T} 243 \mathrm{~A}}$ at the single cellular level. This might give a better insight in the induced activity of this mutant. 


\subsection{Role of phosphorylation on Ptf1a on its activity}

Phosphorylation by Cdks on S/T-P sites in proneural bHLH transcription factors like Neurod4, Ascl1 and Neurog2 regulates their activity in inducing neuronal differentiation (Ali et al., 2011; Ali et al., 2014; Hardwick and Philpott, 2015). Although Ptf1a also contains multiple S/T-P sites, an increase in the induction of pan-neuronal marker genes could be detected when overexpressing murine $\mathrm{Ptf} 1 \mathrm{a}^{8 \mathrm{~S} / \mathrm{T} \rightarrow \mathrm{A}}$ in animal caps compared to overexpression of the wild-type murine Ptf1a (Richts, 2013), indicating that strength of Ptf1a activity might not be regulated by Cdk dependent phosphorylation. However, as previously reported (Richts, 2013), the murine Ptf1a constructs in general tend to have a stronger neuronal inducing activity compared to the $X$. laevis Ptf1a when overexpressed in $X$. laevis embryos. This would mean that potential differences in the strength of induction of neuronal differentiation may be masked by the general increase in activity of the murine Ptf1a constructs.

Mutation of a threonine residue to alanine in the Ptf1a $\mathrm{C} 2$ domain resulted in the up-regulation of marker genes for both GABAergic and glutamatergic cell fates. This change in the neuronal transmitter phenotype was conserved between the murine and the $X$. laevis Ptf1a, indicating this residue is required and sufficient in the regulation of the induced neuronal subtype. Mutations in a single serine residue in the second helix domain for bHLH transcription factors such as Ascl1 and Neurog2 disrupts their activity during neuronal subtype specification by affecting their binding to certain promoter regions (Ali et al., 2011; Hindley et al., 2012; Ali et al., 2014; Quan et al., 2016). However, as the C2 domain in Ptf1a is well characterized for the interaction with Rbpj (Beres et al., 2006; Hori et al., 2008), such a mechanism is rather unlikely as being responsible for the mixed induced neuronal transmitter phenotype. Interestingly, the neuronal differentiation promoting activity of Ptf1 $\mathrm{a}^{\mathrm{T} 243 \mathrm{~A}}$ was reduced compared to the wild-type Ptf1a, a phenotype similar to those of the Ptf1aW2424A/W224A mutant. However, the decreased ability to induce neuronal differentiation is unlikely the reason for the switch in the induced neuronal subtype, as the mixed neuronal subtype induced by $\mathrm{Ptf}_{1} \mathrm{a}^{\mathrm{T} 243 \mathrm{~A}}$ can still be observed even when normalized for the various strength of neuronal differentiation inducing activity. The differences in the ability of the different 
Ptf1a mutants to promote neuronal differentiation might occur due to differences in the protein stability of those mutants. Protein stability was not directly measured and would be of interest to determine, but the similar BiFC signal observed for all mutants with E12 is not indicative of dramatically differences in stability. However, it should be noted that the concentration used for the BiFC studies was more than 10-fold higher than used in the ectodermal explant assays.

Direct evidence that Ptf1a is indeed phosphorylated on the $\mathrm{T}_{243}$ position is still missing. However, overexpression of the phosphomimic mutant ( $\mathrm{Ptf}^{\mathrm{T} 243 \mathrm{E}}$ ) also induces a mixed neuronal transmitter phenotype. The activity of Ptf1 ${ }^{\text {T243E }}$ was more similar to the activity of wild-type Ptf1a indicating that the presence of a negative charge at this position may influence the correct neuronal subtype inducing activity, supporting a role for phosphorylation at this residue. Interestingly, similar to the $\mathrm{Ptf} 1^{1243 \mathrm{~A}}$ mutant, the $\mathrm{Ptf} 1^{\mathrm{T} 243 \mathrm{E}}$ still strongly interacts with Prdm13 in BiFC assays. This may explain why the induced neuronal transmitter phenotype by Ptf1 ${ }^{\text {T243E }}$ is still mixed, even if it resembles more the wild-type Ptf1a than Ptf1 ${ }^{\mathrm{T} 243 \mathrm{~A}}$ in GABAergic gene induction. On the other hand, the negative charge introduced by a single phosphomimic mutation is less strong than a phosphorylated residue (Strickfaden et al., 2007; Pearlman et al., 2011). So, a complete rescue might not be possible by introducing just a single glutamate residue and the introduction of two adjacent phosphomimic mutations may be a more suitable construct (Strickfaden et al., 2007; Pearlman et al., 2011). In future, it would be interesting to provide evidence that the phosphorylation on the $T_{243}$ residue occurs during neurogenesis on Ptf1a to gain a better understanding if phosphorylation indeed regulates Ptf1a activity.

\subsection{BAF complex mediated chromatin remodeling is important for the activation of delayed target gene expression by Ptf1a}

The BAF chromatin remodeling complex has been demonstrated to be essential for vertebrate neurogenesis (Seo et al., 2005; Narayanan et al., 2015; Bachmann et al., 2016). A knock-down of the BAF catalytic core unit Brg1 
impairs neuronal differentiation induced by proneural transcription factors like Neurog2 and Neurod1 (Seo et al., 2005). Further supporting the importance of a functional BAF complex for neurogenesis, Ptf1a-induced neuronal differentiation is also impaired by a knock-down of $\mathrm{Brg} 1$ in $X$. laevis ectodermal explants. Considering the temporal induction of target genes by Ptf1a, only a few direct Ptf1a target genes were affected after $3 \mathrm{~h}$ ( 4 out of 22 genes), while the amount of affected direct target genes increases for delayed direct target genes (12 h, 7 out of 10 genes). Even if this tendency might be the result of a bias in the selection of target genes studied by Nanostring analysis, indications exist that a functional Brg1 is required for the induction of at least a subset of delayed Ptf1a target genes. A similar effect has been shown during myogenesis, where BAF complex dependent chromatin remodeling is required for the activation of only a subset of target genes involved in muscle differentiation (de la Serna et al., 2001; Roy et al., 2002; Berkes et al., 2004). However, the general effect of a Brg1 knock-down on the activation of target genes by Ptf1a was quite low (53 out of 819 genes). Nevertheless, the finding that the most strongly affected genes were indirect Ptf1a target genes indicates that additional mechanism are responsible for the delayed activation of target genes by Ptf1a. Furthermore, besides of its role in remodeling chromatin, the subunits of BAF complex can directly interact with other proteins like transcription factors and so activate or inhibit their function (Zhan et al., 2011; Ninkovic et al., 2013). It would be of interest for future studies to compare Ptf1a overexpressing ectopic explants in presence of a Brg1 morpholino by ATAC-seq to demonstrate that the effects Brg1 knockdown on the upregulations of the identified genes is indeed due to chromatin remodeling. However, since it has been reported that a knock-down of Brg1 cannot completely abolish Brg1 activity due to putative maternal expression ( $\mathrm{Li}$ et al., 2013) and the fact that a down-regulation of Brg1 is associated with cell-cycle arrest and so lethal for the cells (Li et al., 2013), meaning that a complete Brg1 activity cannot be degraded without killing the cells, suggesting that a higher number of genes dependent on Brg1 might exist. Furthermore, the BAF chromatin remodeling complex can also contain Brm instead of Brg1 as catalytic subunit, so that activation of several target genes might not be 
dependent on Brg1 but on Brm instead. Performing a double-knock-down of Brg1 and Brm would give new insights in this matter.

\subsection{Ptf1a does not need open chromatin to induce its targets}

Active transcription of target genes has been associated with accessible chromatin at target genes regulatory regions. It has been reported that chromatin regions of many proneural genes become accessible before their transcription starts (Chen and Dent, 2014; Hontelez et al., 2015; Mo et al., 2015). Here, it has been demonstrated that Ptf1a is able to cause changes in the state of chromatin of some of its direct targets, although it has not been shown if this is a direct or indirect effect. However, most of investigated direct Ptf1a target genes lay in quite closed chromatin regions, whose pattern did not change over time or in dependency of Ptf1a. Since the quality of the ATAC sequencing library was impaired due to redundancy of sequences, these results should be viewed caution. Additional open chromatin regions in those target genes might be detected in samples with more unique reads. Nevertheless, the results indicate that Ptf1a does not need open chromatin to drive the activation of its target genes. This is supported by the fact, that other proneural bHLH transcription factors like Neurod1, Ascl1 and Neurog2 can bind to closed chromatin regions to induce the expression of their target genes (Soufi et al., 2015; Pataskar et al., 2016; Smith et al., 2016). In parallel to the onset of gene expression, the proneural genes induce changes in the chromatin pattern, which then lead to a stabilization of transcription. Studies investigating the distribution of maternally provided epigenetic marks in $X$. tropicalis indicate that many marks for enhancer elements are zygotically provided and are located in regions characterized by repressive histone marks and high levels of methylated DNA (Hontelez et al., 2015), further supporting the idea that Ptf1a is a pioneer transcription factor, which does not need open chromatin region to activate transcription of their target genes. On the other hand, it was reported that the promoter regions of late transcribed genes are already poised with activating histone marks and often are hypomethylated at a very early stage of the embryo (Hontelez et al., 2015) and that the presence of active marks is not sufficient to drive target gene transcription (Hontelez et 
al., 2015). A similar effect could also be detected in the ATAC sequencing since for most of the Ptf1a target genes, open chromatin regions could be found at the transcriptional start site of the genes, while the remaining regions were in general not accessible, supporting the idea that open chromatin is not sufficient for the onset of target gene transcription by Ptf1a. However, whether Ptf1a also opens the chromatin of most of its target genes like Neurod1 (Pataskar et al., 2016) remains unclear. After $12 \mathrm{~h}$ of Ptf1a induction, such a phenomenon could not be detected. But as also early expressed Ptf1a target genes were mostly located in closed chromatin regions, which did not change at the later stage, it might be that Ptf1a activates its target genes without opening the chromatin. However, at this time point, the expression of delayed target genes has just started, so that a change in the chromatin might not be detectable at this stage. Performing ATAC sequencing at a later stage after Ptf1a induction would give more insights in this question.

\subsection{Mechanisms driving the delayed activation of target genes}

Nanostring and RNA-seq data from Ptf1a overexpressing animal caps indicate that Ptf1a activates its direct target genes at different time points. Interestingly, by comparing the induced target genes with a list of genes being activated by Ptf1a in the presence of the translational inhibitor CHX (Schneider-Poetsch et al., 2010), the time of late target gene induction could be detected at a $12 \mathrm{~h}$ timepoint after gene induction. This indicates that certain events have to take place leading to the activation of first delayed target genes.

Interestingly, delayed target gene activation correlates with the timing of $t u b b 2 b$ activation. As tubb2b is a marker for post-mitotic neurons (Oschwald et al., 1991), indicating that the timing of delayed target gene activation may correlate with the exit of the cell cycle. Considering that direct target genes having their onset after $12 \mathrm{~h}$ of Ptf1a induction and are highly enriched for functions in neuronal specification, while early activated genes are more associated with regulation of transcription and the regulation of the cell cycle, would support such a mechanism. Together this would mean that cell cycle escape triggers the events leading to the activation of late target genes. 
However, how cell cycle escape can be associated with the delayed expression of Ptf1a late target genes need to be further analyzed. It has been reported that the length of the cell cycle plays an essential role in neuronal differentiation, as an elongation of the cell cycle is required for inducing the onset of differentiation by proneural bHLH transcription factors (Lange et al., 2009; Lange and Calegari, 2010). Furthermore, the activity of many bHLH transcription factors involved in promoting neuronal differentiation including Neurog2 or Neurod4, are highly regulated by the cell cycle through Cdkmediated phosphorylation (Ali et al., 2011; Hindley et al., 2012; Hardwick and Philpott, 2015). As Ptf1a also contains residues that would make it a candidate for Cdk-dependent phosphorylation, a cell cycle dependent regulation of Ptf1a activity cannot be excluded.

Chromatin remodeling has been reported to regulate transcription during development since actively transcribed genes are associated with accessible chromatin as well as activating histone marks and demethylated DNA (Yao and Jin, 2014; Hsieh and Zhao, 2016; Yao et al., 2016). However, the findings in ATAC-seq that Ptf1a target genes are located within rather closed chromatin regions suggests that chromatin remodeling plays a minor role in the delayed activation of Ptf1a target genes. This is further supported by the fact that the presence of open chromatin marks is not sufficient for inducing transcription (Hontelez et al., 2015) and that high levels of DNA methylation, which is associated with transcriptional silencing, does not affect the transcription of target genes in the early embryo (Bogdanovic et al., 2012). Considering that especially late Ptf1a target genes need Brg1 to be expressed, while induction of most direct early Ptf1a target genes is not affected, BAF complex mediated chromatin remodeling still has an influence on late target gene activation. The finding that Brg1 is required for the activation of several of the late Ptf1 a direct target genes would support an implement of cell cycle regulation in Ptf1a late target gene activation. This is further supported by $\mathrm{Brg} 1$ has been reported to be involved in the regulation of cell cycle exit via interaction with Geminin (Seo et al., 2005). This could also explain that early Ptf1a target genes are mostly unaffected by a Brg1 knockdown, because as those genes are mostly involved in transcriptional and cell cycle regulation, their activity is required before cell cycle exit, which is regulated by Brg1. Nevertheless, chromatin remodeling 
alone cannot explain the delayed activation of late target genes, indicating that several mechanisms drive the delate activation of target genes. A potential mechanism might be the presence or absence of a certain Co-factor or repressor regulating the transcription of those genes. Such a possibility would be supported by findings that the onset of late Ptf1a target gene activation is preponed in presence of CHX (Hedderich, 2012).

Together, these indications lead to a model that Ptf1a induces at least two waves of target gene activation. The first one is activated nearly immediately and mostly controls the transcription of genes controlling the differentiation of the cell to a neuronal cell fate, which leads to a cell cycle escape. An exception is Prdm13, which allows for an early bias of the progenitor cell to a GABAergic cell fate. At the time of cell cycle arrest, a second wave of target genes are activated, which further regulate the subtype specification and function of the induced neuron. Activation of these genes is regulated by several mechanisms. BAF complex mediated chromatin remodeling is required for the activation of a subset of those genes, while another unknown mechanism like the presence an unknown Co-factor or repressor controls the expression of the other late target genes. This would mean that investigating the Ptf1a transcription factor complex at the early and the late time point would be interesting to identify which differences occur in the composition of these complexes between those two time points. 


\section{Bibliography}

Aaku-Saraste, E., et al. (1996). "Loss of occludin and functional tight junctions, but not ZO-1, during neural tube closure-remodeling of the neuroepithelium prior to neurogenesis." Dev Biol 180(2): 664-679.

Afelik, S., et al. (2006). "Combined ectopic expression of Pdx1 and $\mathrm{Ptf} 1 \mathrm{a} / \mathrm{p} 48$ results in the stable conversion of posterior endoderm into endocrine and exocrine pancreatic tissue." Genes Dev 20(11): 1441-1446. Ahnfelt-Rønne, J., et al. (2012). "Ptf1 a control of DIl1 reveals an alternative to the lateral inhibition mechanism." Development 139: 33-45.

Al-Shammari, M., et al. (2011). "A novel PTF1A mutation in a patient with severe pancreatic and cerebellar involvement." Clin Genet 80(2): 196-198.

Ali, F., et al. (2011). "Cell cycle-regulated multi-site phosphorylation of Neurogenin 2 coordinates cell cycling with differentiation during neurogenesis." Development 138(19): 4267-4277.

Ali, F. R., et al. (2014). "The phosphorylation status of Ascl1 is a key determinant of neuronal differentiation and maturation in vivo and in vitro." Development 141(11): 2216-2224.

Allain, A. E., et al. (2011). "Maturation of the GABAergic transmission in normal and pathologic motoneurons." Neural Plast 2011: 905624.

Bachmann, C., et al. (2016). "mSWI/SNF (BAF) Complexes Are Indispensable for the Neurogenesis and Development of Embryonic Olfactory Epithelium." PLoS Genet 12(9): e1006274.

Banik, A., et al. (2017). "Maternal Factors that Induce Epigenetic Changes Contribute to Neurological Disorders in Offspring." Genes 8(6): 150.

Bao, Y. and X. Shen (2007). "INO80 subfamily of chromatin remodeling complexes." Mutation Research/Fundamental and Molecular Mechanisms of Mutagenesis 618(1): 18-29.

Beatus, P. and U. Lendahl (1998). "Notch and neurogenesis." Journal of neuroscience research 54(2): 125-136.

Bellefroid, E. J., et al. (1996). "X-MyT1, a Xenopus C2HC-type zinc finger protein with a regulatory function in neuronal differentiation." Cell 87: 11911202.

Bellefroid, E. J., et al. (1998). "Xiro3 encodes a Xenopus homolog of the Drosophila Iroquois genes and functions in neural specification." EMBO J. 17(1): 191-203.

Beres, T. M., et al. (2006). "PTF1 is an organ-specific and Notchindependent basic helix-loop-helix complex containing the mammalian Suppressor of Hairless (RBP-J) or its paralogue, RBP-L." Mol Cell Biol 26(1): 117-130.

Berkes, C. A., et al. (2004). "Pbx marks genes for activation by MyoD indicating a role for a homeodomain protein in establishing myogenic potential." Molecular cell 14(4): 465-477.

Bernstein, B. E., et al. (2007). "The mammalian epigenome." Cell 128(4): 669-681.

Bertrand, N., et al. (2002). "Proneural genes and the specification of neural cell types." Nat Rev Neurosci 3(7): 517-530.

Bessodes, N., et al. (2017). "Prdm13 forms a feedback loop with Ptf1a and is required for glycinergic amacrine cell genesis in the Xenopus Retina." Neural Dev 12(1): 16. 
Bogdanovic, O., et al. (2012). "The epigenome in early vertebrate development." Genesis 50(3): 192-206.

Bonev, B., et al. (2012). "MicroRNA-9 modulates Hes1 ultradian oscillations by forming a double-negative feedback loop." Cell reports 2(1): 10-18.

Borchers, A. and T. Pieler (2010). "Programming pluripotent precursor cells derived from Xenopus embryos to generate specific tissues and organs." Genes 1(3): 413-426.

Borodinsky, L. N. (2017). "Xenopus laevis as a Model Organism for the Study of Spinal Cord Formation, Development, Function and Regeneration." Frontiers in Neural Circuits 11(90).

Borromeo, M. D., et al. (2014). "A transcription factor network specifying inhibitory versus excitatory neurons in the dorsal spinal cord." Development 141(14): 2803-2812.

Bouwmeester, T., et al. (1996). "Cerberus is a head-inducing secreted factor expressed in the anterior endoderm of Spemann's organizer." Nature 382(6592): 595-601.

Bowes, J. B., et al. (2007). "Xenbase: a Xenopus biology and genomics resource." Nucleic Acids Res 36(suppl_1): D761-D767.

Bray, S. J. (2006). "Notch signalling: a simple pathway becomes complex." Nature reviews Molecular cell biology 7(9): 678-689.

Brewster, R., et al. (1998). "Gli/Zic factors pattern the neural plate by defining domains of cell differentiation." Nature 393(6685): 579-583.

Briscoe, J. and J. Ericson (2001). "Specification of neuronal fates in the ventral neural tube." Current Opinion in Neurobiology 11: 43-49.

Briscoe, J., et al. (2000). "A Homeodomain Protein Code Specifies Progenitor Cell Identity and Neuronal Fate in the Ventral Neural Tube." Cell 101: 435-445.

Buenrostro, J. D., et al. (2013). "Transposition of native chromatin for fast and sensitive epigenomic profiling of open chromatin, DNA-binding proteins and nucleosome position." Nat Methods 10(12): 1213-1218.

Burgess, R., et al. (1995). "Paraxis: a basic helix-loop-helix protein expressed in paraxial mesoderm and developing somites." Dev Biol 168(2).

Castro, D. S., et al. (2011). "A novel function of the proneural factor Ascl1 in progenitor proliferation identified by genome-wide characterization of its targets." Genes Dev 25(9): 930-945.

Castro, D. S., et al. (2006). "Proneural bHLH and Brn proteins coregulate a neurogenic program through cooperative binding to a conserved DNA motif." Dev Cell 11(6): 831-844.

Cau, E., et al. (2002). "Mash1 and Ngn1 control distinct steps of determination and differentiation in the olfactory sensory neuron lineage." Development 129(8): 1871-1880.

Cau, E., et al. (1997). "Mash1 activates a cascade of bHLH regulators in olfactory neuron progenitors." Development 124(8): 1611-1621.

Cedar, H. and Y. Bergman (2009). "Linking DNA methylation and histone modification: patterns and paradigms." Nature Reviews Genetics 10(5): 295304.

Chang, J. C., et al. (2013). "Prdm13 mediates the balance of inhibitory and excitatory neurons in somatosensory circuits." Dev Cell 25(2): 182-195.

Chen, T. and S. Y. Dent (2014). "Chromatin modifiers and remodellers: regulators of cellular differentiation." Nat Rev Genet 15(2): 93-106. 
Chitnis, A. and C. Kintner (1995). "Neural induction and neurogenesis in amphibian embryos. ." Perspect Dev Neurobiol 3(1): 3-15.

Cockell, M., et al. (1989). "Identification of a cell-specific DNA-binding activity that interacts with a transcriptional activator of genes expressed in the acinar pancreas." Mol Cell Biol 9(6): 2464-2476.

Cserjesi, P., et al. (1995). "Scleraxis: a basic helix-loop-helix protein that prefigures skeletal formation during mouse embryogenesis." Development 121(4): 1099-1110.

Davis, R. L. and D. L. Turner (2001). "Vertebrate hairy and Enhancer of split related proteins: transcriptional repressors regulating cellular differentiation and embryonic patterning." Oncogene 20(58): 8342-8357.

Dawson, S. R., et al. (1995). "Specificity for the hairy/enhancer of split basic helix-loop-helix (bHLH) proteins maps outside the bHLH domain and suggests two separable modes of transcriptional repression." Mol Cell Biol 15(12): 6923-6931.

de la Calle-Mustienes, E., et al. (2002). "Xiro homeoproteins coordinate cell cycle exit and primary neuron formation by upregulating neuronal-fate repressors and downregulating the cell-cycle inhibitor XGadd45-y." Mech Dev 119(1): 69-80.

de la Serna, I. L., et al. (2001). "MyoD Can Induce Cell Cycle Arrest but Not Muscle Differentiation in the Presence of Dominant Negative SWI/SNF Chromatin Remodeling Enzymes." Journal of Biological Chemistry 276(44): 41486-41491.

De Robertis, E. M. and H. Kuroda (2004). "Dorsal-Ventral Patterning and Neural Induction in Xenopus Embryos." Annu Rev Cell Dev Biol 20: 285-308. Delgado-Morales, R., et al. (2017). "Epigenetic mechanisms during ageing and neurogenesis as novel therapeutic avenues in human brain disorders." Clinical epigenetics 9(1): 67.

Dobin, A., et al. (2013). "STAR: ultrafast universal RNA-seq aligner." Bioinformatics 29(1): 15-21.

Droz, S. T. and K. A. McLaughlin (2017). Use of Xenopus Frogs to Study Renal Development/Repair. Kidney Development and Disease. R. K. Miller. Cham, Springer International Publishing: 77-107.

Dubey, A. and J.-P. Saint-Jeannet (2017). "Modeling Human Craniofacial Disorders in Xenopus." Current Pathobiology Reports 5(1): 79-92.

Dubois, L., et al. (1998). "XCoe2, a transcription factor of the Col/Olf-1/EBF family involved in the specification of primary neurons in Xenopus." Curr Biol 8(4): 199-209.

Dullin, J. P., et al. (2007). "Ptf1a triggers GABAergic neuronal cell fates in the retina." BMC Dev Biol 7: 110.

Durak, O., et al. (2016). "Chd8 mediates cortical neurogenesis via transcriptional regulation of cell cycle and Wnt signaling." Nat Neurosci.

Egan, C. M., et al. (2013). "CHD5 is required for neurogenesis and has a dual role in facilitating gene expression and polycomb gene repression." Dev Cell 26(3): 223-236.

Ellis, P., et al. (2004). "SOX2, a persistent marker for multipotential neural stem cells derived from embryonic stem cells, the embryo or the adult." Developmental neuroscience 26(2-4): 148-165.

Farah, M. H., et al. (2000). "Generation of neurons by transient expression of neural bHLH proteins in mammalian cells." Development 127: 693-702. 
Feng, J., et al. (2012). "Identifying ChIP-seq enrichment using MACS." Nat Protoc 7(9): 1728-1740.

Fode, C., et al. (2000). "A role for neural determination genes in specifying the dorsoventral identity of telencephalic neurons." Genes and Development 14: 67-80.

Fujitani, Y., et al. (2006). "Ptf1a determines horizontal and amacrine cell fates during mouse retinal development." Development 133(22): 4439-4450.

Gammill, L. S. and H. Sive (1997). "Identification of otx2 target genes and restrictions in ectodermal competence during Xenopus cement gland formation." Development 124(2): 471-481.

Gao, S. (2017). "Epigenetic regulation of somatic cell reprogramming Yixuan Wang, Yan Bi and Shaorong Gao." Current opinion in genetics \& development 46: 156-163.

Gao, Z., et al. (2009). "Neurod1 is essential for the survival and maturation of adult-born neurons." Nat Neurosci 12(9): 1090.

Gaspar-Maia, A., et al. (2009). "Chd1 regulates open chromatin and pluripotency of embryonic stem cells." Nature 460(7257): 863-868.

Glasgow, S. M., et al. (2005). "Ptf1a determines GABAergic over glutamatergic neuronal cell fate in the spinal cord dorsal horn." Development 132(24): 5461-5469.

Gomez-Skarmeta, J. L., et al. (1998). "Xiro, a Xenopus homolog of the Drosophila Iroquois complex genes, controls development at the neural plate." EMBO J. 17(1): 181-190.

Goodwin, L. R. and D. J. Picketts (2017). "The role of ISWI chromatin remodeling complexes in brain development and neurodevelopmental disorders." Molecular and Cellular Neuroscience.

Gowan, K., et al. (2001). "Crossinhibitory Activities of Ngn1 and Math1 Allow Specification of Distinct Dorsal Interneurons." Neuron 31(2): 219-232.

Graham, V., et al. (2003). "SOX2 functions to maintain neural progenitor identity." Neuron 39(5): 749-765.

Gross, M. K., et al. (2002). "Lbx1 Specifies Somatosensory Association Interneurons in the Dorsal Spinal Cord." Neuron 34: 535-549.

Groves, A. K. and C. LaBonne (2014). "Setting appropriate boundaries: fate, patterning and competence at the neural plate border." Dev Biol 389(1): 2-12.

Guénette, S. A., et al. (2013). "Pain Perception and Anaesthesia in Research Frogs." Experimental Animals 62(2): 87-92.

Hamburger, V. (1969). "Hans Spemann and the organizer concept." Cellular and Molecular Life Sciences 25(11): 1121-1125.

Hanotel, J., et al. (2014). "The Prdm13 histone methyltransferase encoding gene is a Ptf1a-Rbpj downstream target that suppresses glutamatergic and promotes GABAergic neuronal fate in the dorsal neural tube." Dev Biol 386(2): 340-357.

Hardcastle, Z., et al. (2000). "FGF-8 stimulates neuronal differentiation through FGFR-4a and interferes with mesoderm induction in Xenopus embryos." Current Biology 10(23): 1511-1514.

Hardwick, L. J., et al. (2015). "Cell cycle regulation of proliferation versus differentiation in the central nervous system." Cell Tissue Res 359(1): 187200. 
Hardwick, L. J. and A. Philpott (2015). "Multi-site phosphorylation regulates NeuroD4 activity during primary neurogenesis: a conserved mechanism amongst proneural proteins." Neural Dev 10: 15.

Harland, R. M. (1991). "In situ hybridization: an improved whole-mount method for Xenopus embryos." Methods Cell Biol 36: 685-695.

Hartenstein, V. (1989). "Early neurogenesis in Xenopus: the spatio-temporal pattern of proliferation and cell lineages in the embryonic spinal cord." Neuron. 3(4): 399-411.

Hartenstein, V. (1993). "Early pattern of neuronal differentiation in the Xenopus embryonic brainstem and spinal cord." Journal of Comparative Neurology 328(2): 213-231.

Heasman, J., et al. (2000). " $\beta$ Catenin Signaling Activity Dissected in the Early Xenopus Embryo: A Novel Antisense Approach." Dev Biol 222(1): 124134.

Hedderich, M. (2008) Diploma Thesis: Charakterisierung der proneuralen Aktivität von Ptf1a/p48 in Xenopus University Medical Center Göttingen, Department of Developmental Biochemistry, University of Göttingen Hedderich, M. (2012). "Doctoral Thesis: Molecular characterization of Ptf1a activity during Xenopus embryogenesis."

Helms, A. W. and J. E. Johnson (2003). "Specification of dorsal spinal cord interneurons." Curr Opin Neurobiol 13(1): 42-49.

Hemmati-Brivanlou, A., et al. (1994). "Follistatin, an Antagonist of Activin, Is Expressed in the Spemann Organizer and Displays Direct Neuralizing Activity." Cell 77: 283-295.

Hemmati-Brivanlou, A. and D. A. Melton (1994). "Inhibition of Activin Receptor Signaling Promotes Neuralization in Xenopus." Cell 77: 273-281. Henke, R. M., et al. (2009). "Neurog2 is a direct downstream target of the Ptf1a-Rbpj transcription complex in dorsal spinal cord." Development 136(17): 2945-2954.

Hernandez-Miranda, L. R., et al. (2017). "The dorsal spinal cord and hindbrain: From developmental mechanisms to functional circuits." Dev Biol 432(1): 34-42.

Hindley, C., et al. (2012). "Post-translational modification of Ngn2 differentially affects transcription of distinct targets to regulate the balance between progenitor maintenance and differentiation." Development 139(10): 1718-1723.

Ho, L. and G. R. Crabtree (2010). "Chromatin remodelling during development." Nature 463(7280): 474-484.

Ho, L., et al. (2009). "An embryonic stem cell chromatin remodeling complex, esBAF, is essential for embryonic stem cell self-renewal and pluripotency."

Proceedings of the National Academy of Sciences 106(13): 5181-5186.

Hollemann, T., et al. (1996). "Xenopus Xsal-1, a vertebrate homolog of the region specific homeotic gene spalt of Drosophila." Mech Dev 55(1): 19-32.

Hontelez, S., et al. (2015). "Embryonic transcription is controlled by maternally defined chromatin state." Nat Commun 6: 10148.

Hori, K., et al. (2008). "A nonclassical bHLH Rbpj transcription factor complex is required for specification of GABAergic neurons independent of Notch signaling." Genes Dev 22(2): 166-178.

Hori, K. and M. Hoshino (2012). "GABAergic neuron specification in the spinal cord, the cerebellum, and the cochlear nucleus." Neural Plast 2012: 921732. 
Horikawa, K., et al. (2006). "Noise-resistant and synchronized oscillation of the segmentation clock." Nature 441(7094): 719-723.

Hoshino, M., et al. (2005). "Ptf1a, a bHLH transcriptional gene, defines GABAergic neuronal fates in cerebellum." Neuron 47(2): 201-213.

Houtmeyers, R., et al. (2013). "The ZIC gene family encodes multifunctional proteins essential for patterning and morphogenesis." $\underline{\text { Cellular and }}$ Molecular Life Sciences 70(20): 3791-3811.

Hoveyda, N., et al. (1999). "Neonatal diabetes mellitus and cerebellar

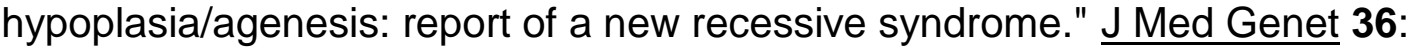
700-704.

Hsieh, J. and X. Zhao (2016). "Genetics and Epigenetics in Adult Neurogenesis." Cold Spring Harb Perspect Biol 8(6).

Huang, M., et al. (2008). "Ptf1a, Lbx1 and Pax2 coordinate glycinergic and peptidergic transmitter phenotypes in dorsal spinal inhibitory neurons." Dev Biol 322(2): 394-405.

Imayoshi, I. and R. Kageyama (2011). "The role of Notch signaling in adult neurogenesis." Molecular neurobiology 44(1): 7-12.

Iskusnykh, I. Y., et al. (2016). "Loss of Ptf1a Leads to a Widespread CellFate Misspecification in the Brainstem, Affecting the Development of Somatosensory and Viscerosensory Nuclei." J Neurosci 36(9): 2691-2710. Jaenisch, R. and A. Bird (2003). "Epigenetic regulation of gene expression: how the genome integrates intrinsic and environmental signals." Nature genetics 33: 245-254.

Jenuwein, T. and C. D. Allis (2001). "Translating the Histone Code." Science 293(5532): 1074-1080.

Jessell, T. M. and J. Dodd (1990). "Floor plate-derived signals and the control of neural cell pattern in vertebrates." Harvey Lect 86: 87-128.

Jones, K. M., et al. (2015). "CHD7 maintains neural stem cell quiescence and prevents premature stem cell depletion in the adult hippocampus." Stem Cells 33(1): 196-210.

Jusuf, P. R., et al. (2012). "Biasing amacrine subtypes in the Atoh7 lineage through expression of Barhl2." Journal of Neuroscience 32(40): 1392913944.

Kadoch, C., et al. (2013). "Proteomic and bioinformatic analysis of mammalian SWI/SNF complexes identifies extensive roles in human malignancy." Nature genetics 45(6): 592-601.

Kageyama, R., et al. (2008). "Dynamic Notch signaling in neural progenitor cells and a revised view of lateral inhibition." Nat Neurosci 11(11): 12471251.

Kanekar, S., et al. (1997). "Xath5 participates in a network of bHLH genes in the developing Xenopus retina." Neuron 19(5): 981-994.

Kani, S., et al. (2010). "Proneural gene-linked neurogenesis in zebrafish cerebellum." Dev Biol 343(1-2): 1-17.

Kawaguchi, Y., et al. (2002). "The role of the transcriptional regulator Ptf1a in converting intestinal to pancreatic progenitors." Nat Genet 32(1): 128-134. Kim, P., et al. (1997). "XATH-1, a Vertebrate Homolog of Drosophila atonal, Induces Neuronal Differentiation within Ectodermal Progenitors." Dev Biol 187: 1-12.

Kiyota, T. and T. Kinoshita (2002). "Cysteine-rich region of X-Serrate-1 is required for activation of Notch signaling in Xenopus primary neurogenesis." International Journal of Developmental Biology 46(8): 1057-1060. 
Kofent, J. and F. M. Spagnoli (2016). "Xenopus as a model system for studying pancreatic development and diabetes." Seminars in Cell \& Developmental Biology 51: 106-116.

Krapp, A., et al. (1996). "The p48 DNA-binding subunit of transcription factor PTF1 is a new exocrine pancreas-specific basic helix-loop-helix protein." EMBO J. 15(16): 4317-4329.

Krapp, A., et al. (1998). "The bHLH protein PTF1-p48 is essential for the formation of the exocrine and the correct spatial organization of the endocrine pancreas." Genes Dev 12(23): 3752-3763.

Kroll, K. L. (2007). "Geminin in embryonic development: coordinating transcription and the cell cycle during differentiation." Front Biosci 12(1395): 409.

Kroll, K. L., et al. (1998). "Geminin, a neuralizing molecule that demarcates the future neural plate at the onset of gastrulation." Development 125(16): 3247-3258.

Kuroda, H., et al. (2004). "Neural induction in Xenopus: requirement for ectodermal and endomesodermal signals via Chordin, Noggin, $\beta$-Catenin, and Cerberus." PLoS biology 2(5): e92.

Lacomme, M., et al. (2012). "NEUROG2 drives cell cycle exit of neuronal precursors by specifically repressing a subset of cyclins acting at the G1 and S phases of the cell cycle." Mol Cell Biol 32(13): 2596-2607.

Lamborghini, J. E. (1980). "Rohon-beard cells and other large neurons in Xenopus embryos originate during gastrulation." Journal of Comparative Neurology 189(2): 323-333.

Lange, C. and F. Calegari (2010). "Cdks and cyclins link G1 length and differentiation of embryonic, neural and hematopoietic stem cells." Cell Cycle 9(10): 1893-1900.

Lange, C., et al. (2009). "Cdk4/cyclinD1 overexpression in neural stem cells shortens $\mathrm{G} 1$, delays neurogenesis, and promotes the generation and expansion of basal progenitors." Cell stem cell 5(3): 320-331.

Langmead, B. and S. L. Salzberg (2012). "Fast gapped-read alignment with Bowtie 2." Nat Methods 9(4): 357-359.

Launay, C., et al. (1996). "A truncated FGF receptor blocks neural induction by endogenous Xenopus inducers." Development 122(3): 869-880.

Le Dréau, G. and E. Martí (2012). "Dorsal-ventral patterning of the neural tube: a tale of three signals." Dev Neurobiol 72(12): 1471-1481.

Leclerc, C., et al. (1997). "L-type calcium channel activation controls the in vivo transduction of the neuralizing signal in the amphibian embryos." Mech Dev 64(1-2): 105-110.

Leclerc, C., et al. (2012). "The calcium: an early signal that initiates the formation of the nervous system during embryogenesis." Frontiers in molecular neuroscience 5: 64.

Leclerc, C., et al. (2000). "Imaging patterns of calcium transients during neural induction in Xenopus laevis embryos." Journal of cell science 113(19): 3519-3529.

Lee-Liu, D., et al. (2017). "The African clawed frog Xenopus laevis: A model organism to study regeneration of the central nervous system." Neuroscience Letters 652: 82-93.

Lee, J. E. (1997). "Basic helix-loop-helix genes in neural development." $\underline{\text { Curr }}$ Opin Neurobiol 7(1): 13-20. 
Lefebvre, V., et al. (2007). "Control of cell fate and differentiation by Sryrelated high-mobility-group box (Sox) transcription factors." The international journal of biochemistry \& cell biology 39(12): 2195-2214.

Lei, Y., et al. (2012). "Efficient targeted gene disruption in Xenopus embryos using engineered transcription activator-like effector nucleases (TALENs)." PNAS 109(43): 17484-17489.

Lelievre, E. C., et al. (2011). "Ptf1a/Rbpj complex inhibits ganglion cell fate and drives the specification of all horizontal cell subtypes in the chick retina." Dev Biol 358(2): 296-308.

Lessard, J., et al. (2007). "An essential switch in subunit composition of a chromatin remodeling complex

during neural development. Neuron. 2007; 55:201-21." Neuron. 55: 201-221. Lewis, J. (1998). Notch signalling and the control of cell fate choices in vertebrates. Seminars in Cell \& Developmental Biology, Elsevier. Li, H., et al. (2011). "Phosphorylation regulates OLIG2 cofactor choice and the motor neuron-oligodendrocyte fate switch." Neuron 69(5): 918-929. Li, H., et al. (2009). "The sequence alignment/map format and SAMtools." Bioinformatics 25(16): 2078-2079.

Li, M., et al. (2006). "The role of early lineage in GABAergic and glutamatergic cell fate determination in Xenopus laevis." J Comp Neurol 495(6): 645-657.

Li, W., et al. (2013). "Brg1 governs distinct pathways to direct multiple aspects of mammalian neural crest cell development." Proceedings of the National Academy of Sciences 110(5): 1738-1743.

Li, Y. and N. E. Baker (2001). "Proneural enhancement by Notch overcomes Suppressor-of-Hairless repressor function in the developing Drosophila eye." Current Biology 11(5): 330-338.

Liem, K. F., Jr.,, et al. (1997). "A role for the roof plate and its resident TGFbeta-related proteins in neuronal patterning in the dorsal spinal cord." Cell 91(1): 127-138.

Lin, H., et al. (2017). "KDM3A-mediated demethylation of histone H3 lysine 9 facilitates the chromatin binding of Neurog2 during neurogenesis."

Development 144(20): 3674-3685.

Lindsell, C. E., et al. (1996). "Expression Patterns ofJagged, Delta1, Notch1, Notch2, andNotch3Genes Identify Ligand-Receptor Pairs That May Function in Neural Development." Molecular and Cellular Neuroscience 8(1): 14-27.

Louvi, A. and S. Artavanis-Tsakonas (2006). "Notch signalling in vertebrate neural development." Nature Reviews Neuroscience 7(2): 93-102.

Love, M. I., et al. (2014). "Moderated estimation of fold change and dispersion for RNA-seq data with DESeq2." Genome Biology 15(12): 550.

Luger, K. and T. J. Richmond (1998). "The histone tails of the nucleosome." Current opinion in genetics \& development 8(2): 140-146.

Ma, Q., et al. (1996). "Identification of neurogenin, a Vertebrate Neuronal Determination Gene." Cell 87.

Ma, Y. C., et al. (2008). "Regulation of motor neuron specification by phosphorylation of neurogenin 2." Neuron 58(1): 65-77.

Masui, T., et al. (2007). "Early pancreatic development requires the vertebrate Suppressor of Hairless (RBPJ) in the PTF1 bHLH complex." Genes Dev 21(20): 2629-2643. 
Masui, T., et al. (2010). "Replacement of Rbpj with Rbpjl in the PTF1 complex controls the final maturation of pancreatic acinar cells."

Gastroenterology 139(1): 270-280.

Masui, T., et al. (2008). "Transcriptional autoregulation controls pancreatic Ptf1a expression during development and adulthood." Mol Cell Biol 28(17): 5458-5468.

Matsumoto, S., et al. (2006). "Brg1 is required for murine neural stem cell maintenance and gliogenesis." Dev Biol 289(2): 372-383.

Mattar, P., et al. (2008). "Basic helix-loop-helix transcription factors cooperate to specify a cortical projection neuron identity." Mol Cell Biol 28(5): 1456-1469.

Maurer, K. A., et al. (2014). "Notch signaling differentially regulates Atoh7 and Neurog2 in the distal mouse retina." Development 141(16): 3243-3254.

Meredith, D. M., et al. (2013). "Program Specificyty for Ptf1a in Pancreas versus Neural Tube Development Correlates with Distinct Collaborating Cofactors and Chromatin Accessability

" Mol Cell Biol 33(16): 3166-3179.

Meredith, D. M., et al. (2009). "Multiple transcriptional mechanisms control Ptf1a levels during neural development including autoregulation by the PTF1J complex." J Neurosci 29(36): 11139-11148.

Millen, K. J., et al. (2014). "Transformation of the cerebellum into more ventral brainstem fates causes cerebellar agenesis in the absence of Ptf1a function." Proc Natl Acad Sci U S A 111(17): E1777-1786.

Mimoto, M. S. and J. L. Christian (2011). "Manipulation of gene function in Xenopus laevis." Vertebrate Embryogenesis: Embryological, Cellular, and Genetic Methods: 55-75.

Mitalipov, S. and D. Wolf (2009). Totipotency, pluripotency and nuclear reprogramming. Engineering of stem cells, Springer: 185-199.

Miyata, Y., et al. (2010). "Cyclin C regulates human hematopoietic stem/progenitor cell quiescence." Stem Cells 28(2): 308-317.

Mizuguchi, R., et al. (2001). "Combinatorial roles of olig2 and neurogenin2 in the coordinated induction of pan-neuronal and subtype-specific properties of motoneurons." Neuron 31(5): 757-771.

Mizuhara, E., et al. (2010). "Purkinje cells originate from cerebellar ventricular zone progenitors positive for Neph3 and E-cadherin." Dev Biol 338(2): 202-214.

Mizuseki, K., et al. (1998). "Xenopus Zic-related-1 and Sox-2, two factors induced by chordin, have distinct activities in the initiation of neural induction." Development 125(4): 579-587.

Mizuseki, K., et al. (1998). "SoxD:an essential mediator of induction of anterior neural tissues in Xenopus embryos." Neuron 21(1): 77-85.

Mo, A., et al. (2015). "Epigenomic Signatures of Neuronal Diversity in the Mammalian Brain." Neuron 86(6): 1369-1384.

Mohn, F., et al. (2008). "Lineage-specific polycomb targets and de novo DNA methylation define restriction and potential of neuronal progenitors." Molecular cell 30(6): 755-766.

Mona, B., et al. (2017). "Repression by PRDM13 is critical for generating precision in neuronal identity." Elife 6.

Mosammaparast, N. and Y. Shi (2010). "Reversal of histone methylation: biochemical and molecular mechanisms of histone demethylases." Annual review of biochemistry 79: 155-179. 
Müller, T., et al. (2002). "The Homeodomain Factor Lbx1 Distinguishes Two Major Programs of Neuronal Differentiation in the Dorsal Spinal Cord."

Neuron 34: 551-562.

Murre, C., et al. (1989). "Interactions between heterologous helix-loop-helix proteins generate complexes that bind specifically to a common DNA sequence." Cell 58(3): 537-544.

Nakata, K., et al. (1997). "Xenopus Zic3, a primary regulator both in neural and neural crest development." Proc Natl Acad Sci U S A 1997(94): 22.

Nakhai, H., et al. (2007). "Ptf1a is essential for the differentiation of GABAergic and glycinergic amacrine cells and horizontal cells in the mouse retina." Development 134(6): 1151-1160.

Narayanan, R., et al. (2015). "Loss of BAF (mSWI/SNF) complexes causes global transcriptional and chromatin state changes in forebrain development." Cell reports 13(9): 1842-1854.

Narayanan, R. and T. C. Tuoc (2014). "Roles of chromatin remodeling BAF complex in neural differentiation and reprogramming." Cell Tissue Res 356(3): 575-584.

Nieber, F., et al. (2009). "Comparative expression analysis of the neurogenins in Xenopus tropicalis and Xenopus laevis." Dev Dyn 238(2): 451-458.

Nieberler, M. (2012) Function of the CHD4/Mi-2ß chromatin remodelling ATPase during neural development of Xenopus laevis

Nieuwkoop, P. D. and J. Faber (1967). "Normal Table of Xenopus laevis(Daudln)." 2nd ed. North Holland Publ. Co.. Amsterdam.

Ninkovic, J., et al. (2013). "The BAF complex interacts with Pax6 in adult neural progenitors to establish a neurogenic cross-regulatory transcriptional network." Cell stem cell 13(4): 403-418.

Nishida, K., et al. (2010). "Ptf1a directly controls expression of immunoglobulin superfamily molecules Nephrin and Neph3 in the developing central nervous system." J Biol Chem 285(1): 373-380.

Nishimura, M., et al. (1998). "Structure, Chromosomal Locus, and Promoter of MouseHes2Gene, a Homologue ofDrosophila hairyandEnhancer of split." Genomics 49(1): 69-75.

Niwa, Y., et al. (2011). "Different types of oscillations in Notch and Fgf signaling regulate the spatiotemporal periodicity of somitogenesis." Genes Dev 25(11): 1115-1120.

Oginuma, M., et al. (2010). "The oscillation of Notch activation, but not its boundary, is required for somite border formation and rostral-caudal patterning within a somite." Development 137(9): 1515-1522.

Ohtsuka, T., et al. (1999). "Hes1 and Hes5 as notch effectors in mammalian neuronal differentiation." EMBO J 18(8): 2196-2207.

Okubo, Y., et al. (2012). "Lfng regulates the synchronized oscillation of the mouse segmentation clock via trans-repression of Notch signalling." Nat Commun 3: 1141.

Oschwald, R., et al. (1991). "Localization of a nervous system-specific class II beta-tubulin gene in Xenopus laevis embryos by whole-mount in situ hybridization." Int J Dev Biol 35: 399-405.

Papalopulu, N. and C. Kintner (1996). "A posteriorising factor, retinoic acid, reveals that anteroposterior patterning controls the timing of neuronal differentiation in Xenopus neuroectoderm." Development 122(11): 34093418. 
Papanayotou, C., et al. (2008). "A mechanism regulating the onset of Sox2 expression in the embryonic neural plate." PLoS biology 6(1): e2.

Parras, C. M., et al. (2002). "Divergent functions of the proneural genes Mash1 and Ngn2 in the specification of neuronal subtype identity." Genes Dev 16(3): 324-338.

Pascual, M., et al. (2007). "Cerebellar GABAergic progenitors adopt an external granule cell-like phenotype in the absence of Ptf1a transcription factor expression." Proc Natl Acad Sci U S A 104(12): 5193-5198.

Pataskar, A., et al. (2016). "NeuroD1 reprograms chromatin and transcription factor landscapes to induce the neuronal program." EMBO J 35(1): 24-45.

Patterson, K. D. and P. A. Krieg (1999). "Hox11-family genes XHox11 and XHox11L2 in xenopus: XHox11L2 expression is restricted to a subset of the primary sensory neurons." Dev Dyn 214(1): 34-43.

Pattyn, A., et al. (2000). "Control of hindbrain motor neuron differentiation by the homeobox gene Phox2b." Development 127(7): 1349-1358.

Pearlman, S. M., et al. (2011). "A Mechanism for the Evolution of Phosphorylation Sites." Cell 147(4): 934-946.

Penzel, R., et al. (2003). "Characterization and early embryonic expression of a neural specific transcription factor xSOX3 in Xenopus laevis." International Journal of Developmental Biology 41(5): 667-677.

Pera, E. M., et al. (2003). "Integration of IGF, FGF, and anti-BMP signals via Smad1 phosphorylation in neural induction." Genes Dev 17(24): 3023-3028.

Pera, E. M., et al. (2001). "Neural and head induction by insulin-like growth factor signals." Dev Cell 1(5): 655-665.

Perron, M., et al. (1999). "X-ngnr-1 and Xath3 promote ectopic expression of sensory neuron markers in the neurula ectoderm and have distinct inducing properties in the retina." Proc Natl Acad Sci U S A 96(26): 14996-15001.

Pevny, L. and M. Placzek (2005). "SOX genes and neural progenitor identity." Current Opinion in Neurobiology 15(1): 7-13.

Pfeuty, B. (2015). "Neuronal specification exploits the inherent flexibility of cell-cycle gap phases." Neurogenesis (Austin) 2(1): e1095694.

Phelan, M. L., et al. (1999). "Reconstitution of a core chromatin remodeling complex from SWI/SNF subunits." Molecular cell 3(2): 247-253.

Piccolo, S., et al. (1999). "The head inducer Cerberus is a multifunctional antagonist of Nodal, BMP and Wnt signals." Nature 397(6721): 707-710.

Piccolo, S., et al. (1996). "Dorsoventral Patterning in Xenopus: Inhibition of Ventral Signals by Direct Binding of Chordin to BMP-4." Cell 86(4): 589-598.

Pitulescu, M., et al. (2005). "The regulation of embryonic patterning and DNA replication by geminin." Cellular and Molecular Life Sciences CMLS 62(13): 1425-1433.

Poduri, A. and D. Lowenstein (2011). "Epilepsy genetics--past, present, and future." Curr Opin Genet Dev 21(3): 325-332.

Pozzoli, O., et al. (2001). "Xebf3 is a regulator of neuronal differentiation during primary neurogenesis in Xenopus." Dev Biol 233(2): 495-512.

Pratt, K. G. and A. S. Khakhalin (2013). "Modeling human neurodevelopmental disorders in the <em $>$ Xenopus $</$ em $>$ tadpole: from mechanisms to therapeutic targets." Disease Models \&amp; Mechanisms. Quan, X.-J., et al. (2016). "Post-translational control of the temporal dynamics of transcription factor activity regulates neurogenesis." Cell 164(3): 460-475. 
Quinlan, A. R. and I. M. Hall (2010). "BEDTools: a flexible suite of utilities for comparing genomic features." Bioinformatics 26(6): 841-842.

Richard-Parpaillon, L., et al. (2004). "G1/S phase cyclin-dependent kinase overexpression perturbs early development and delays tissue-specific differentiation in Xenopus." Development 131(11): 2577-2586.

Richard-Parpaillon, L., et al. (2002). "The IGF pathway regulates head formation by inhibiting Wnt signaling in Xenopus." Dev Biol 244(2): 407-417. Richts, S. (2013) Master's Thesis: Characterization of Ptf1a protein domains during neurogenesis University Medical Center Göttingen, Department of Developmental Biochemistry, University of Göttingen

Roberts, A. (2000). "Early functional organization of spinal neurons in developing lower vertebrates." Brain research bulletin 53(5): 585-593.

Roberts, A., et al. (2012). "A functional scaffold of CNS neurons for the vertebrates: the developing Xenopus laevis spinal cord." Dev Neurobiol 72(4): 575-584.

Rodolosse, A., et al. (2009). "p/CAF modulates the activity of the transcription factor p48/Ptf1a involved in pancreatic acinar differentiation." Biochem J 418(2): 463-473.

Roese-Koerner, B., et al. (2016). "Reciprocal Regulation between Bifunctional miR-9/9* and its Transcriptional Modulator Notch in Human Neural Stem Cell Self-Renewal and Differentiation." Stem Cell Reports 7(2): 207-219.

Roese-Koerner, B., et al. (2017). "Notch/Hes signaling and miR-9 engage in complex feedback interactions controlling neural progenitor cell proliferation and differentiation." Neurogenesis (Austin) 4(1): e1313647.

Rogers, C. D., et al. (2011). "The response of early neural genes to FGF signaling or inhibition of BMP indicate the absence of a conserved neural induction module." BMC Dev Biol 11(1): 74.

Rogers, C. D., et al. (2009). "Xenopus Sox3 activates sox2 and geminin and indirectly represses Xvent2 expression to induce neural progenitor formation at the expense of non-neural ectodermal derivatives." Mech Dev 126(1-2): 42-55.

Ronan, J. L., et al. (2013). "From neural development to cognition: unexpected roles for chromatin." Nat Rev Genet 14(5): 347--359.

Rossi, C. C., et al. (2008). "Rohon-Beard sensory neurons are induced by BMP4 expressing non-neural ectoderm in Xenopus laevis." Dev Biol 314(2): 351-361.

Rossi, C. C., et al. (2009). "Transcriptional control of Rohon-Beard sensory neuron development at the neural plate border." Developmental Dynamics 238(4): 931-943.

Roux, E., et al. (1989). "The cell-specific transcription factor PTF1 contains two different subunits that interact with the DNA." Genes Dev 3(10): 16131624.

Roy, K., et al. (2002). "The myogenic basic helix-loop-helix family of transcription factors shows similar requirements for SWI/SNF chromatin remodeling enzymes during muscle differentiation in culture." Journal of Biological Chemistry 277(37): 33818-33824.

Saka, Y., et al. (2007). "Nuclear accumulation of Smad complexes occurs only after the midblastula transition in Xenopus." Development 134(23): 4209-4218. 
Sakamoto, l., et al. (2000). "A novel $\beta$-catenin-binding protein inhibits $\beta$ catenin-dependent Tcf activation and axis formation." Journal of Biological Chemistry 275(42): 32871-32878.

Sanes, D. H., et al. (2011). Development of the nervous system, Academic Press.

Sasai, Y., et al. (1992). "Two mammalian helix-loop-helix factors structurally related to Drosophila hairy and Enhancer of split." Genes Dev 6(12b): 26202634.

Sasai, Y., et al. (1996). "Endoderm induction by the organizer-secreted factors chordin and noggin in Xenopus animal caps." EMBO J. 15(17): 45474555.

Schlosser, G., et al. (2002). "Thyroid hormone promotes neurogenesis in the Xenopus spinal cord." Dev Dyn 225(4): 485-498.

Schneider-Poetsch, T., et al. (2010). "Inhibition of eukaryotic translation elongation by cycloheximide and lactimidomycin." Nature chemical biology 6(3): 209-217.

Schneider, M. L., et al. (2001). "Notch signaling can inhibit Xath5 function in the neural plate and developing retina." Molecular and Cellular Neuroscience 18(5): 458-472.

Schnetz, M. P., et al. (2009). "Genomic distribution of CHD7 on chromatin tracks H3K4 methylation patterns." Genome Res 19(4): 590-601.

Schroeter, E. H., et al. (1998). "Notch-1 signalling requires ligand-induced proteolytic release of intracellular domain." Nature 393(6683): 382-386.

Selkoe, D. and R. Kopan (2003). "Notch and Presenilin: regulated intramembrane proteolysis links development and degeneration." Annual review of neuroscience 26(1): 565-597.

Sellick, G. S., et al. (2004). "Mutations in PTF1A cause pancreatic and cerebellar agenesis." Nat Genet 36(12): 1301-1305.

Seo, S. and K. L. Kroll (2006). "Geminin's double life: chromatin connections that regulate transcription at the transition from proliferation to differentiation." Cell Cycle 5(4): 374-379.

Seo, S., et al. (2007). "Neurogenin and NeuroD direct transcriptional targets and their regulatory enhancers." EMBO J 26: 5093-5108.

Seo, S., et al. (2005). "The SWI/SNF chromatin remodeling protein Brg1 is required for vertebrate neurogenesis and mediates transactivation of $\mathrm{Ngn}$ and NeuroD." Development 132(1): 105-115.

Sharp, P. A., et al. (1973). "Detection of two restriction endonuclease activities in Haemophilus parainfluenzae using analytical agarose--ethidium bromide electrophoresis." Biochemistry 12(16): 3055-3063.

Shen, T., et al. (2015). "CHD2 is required for embryonic neurogenesis in the developing cerebral cortex." Stem Cells 33(6): 1794-1806.

Shimojo, H., et al. (2016). "Oscillatory control of Delta-like1 in cell interactions regulates dynamic gene expression and tissue morphogenesis." Genes and Development 30(1): 102-116.

Shimojo, H., et al. (2008). "Oscillations in notch signaling regulate maintenance of neural progenitors." Neuron 58(1): 52-64.

Simmen, M. W. (2008). "Genome-scale relationships between cytosine methylation and dinucleotide abundances in animals." Genomics 92(1): 3340.

Sive, H. L., et al. (2000). "Early Development of Xenopus laevis - A Laboratory Manual." Cold Spring Harb Laboratory Press London. 
Smith, D. K., et al. (2016). "Small Molecules Modulate Chromatin Accessibility to Promote NEUROG2-Mediated Fibroblast-to-Neuron Reprogramming." Stem Cell Reports 7(5): 955-969.

Sokpor, G., et al. (2017). "Chromatin Remodeling BAF (SWI/SNF) Complexes in Neural Development and Disorders." Frontiers in molecular neuroscience 10: 243.

Sölter, M., et al. (1999). "Characterization of a subfamily of related winged helix genes, XFD-12/12'/12 "(XFLIP), during Xenopus embryogenesis." Mech Dev 89(1): 161-165.

Sommer, L., et al. (1996). "neurogenins, a novel family of atonal-related bHLH transcription factors, are Putative mammalian neuronal determination genes that reveal progenitor cell heterogeneity in the developing CNS and PNS." Molecular and Cellular Neuroscince 8: 221-241.

Soufi, A., et al. (2015). "Pioneer transcription factors target partial DNA motifs on nucleosomes to initiate reprogramming." Cell 161(3): 555-568. Souopgui, J., et al. (2002). "Xebf3 is a regulator of neuronal differentiation during primary neurogenesis in Xenopus." Dev Biol 233(2): 495-512.

Stancheva, I. and R. R. Meehan (2000). "Transient depletion of xDnmt1 leads to premature gene activation in Xenopus embryos." Genes Dev 14(3): 313-327.

Storm, R., et al. (2009). "The bHLH transcription factor Olig3 marks the dorsal neuroepithelium of the hindbrain and is essential for the development of brainstem nuclei." Development 136(2): 295-305.

Strahl, B. D. and C. D. Allis (2000). "The language of covalent histone modifications." Nature 403(6765): 41-45.

Strickfaden, S. C., et al. (2007). "A Mechanism for Cell Cycle Regulation of MAP Kinase Signaling in a Yeast Differentiation Pathway." Cell 128(3): 519531.

Sullivan, S. A., et al. (2001). "foxD5a, a Xenopus winged helix gene, maintains an immature neural ectoderm via transcriptional repression that is dependent on the C-terminal domain." Dev Biol 232(2): 439-457.

Thuret, R., et al. (2015). "Analysis of neural progenitors from embryogenesis to juvenile adult in Xenopus laevis reveals biphasic neurogenesis and continuous lengthening of the cell cycle." Biol Open 4(12): 1772-1781.

Tuoc, T. C., et al. (2013). "Chromatin regulation by BAF170 controls cerebral cortical size and thickness." Dev Cell 25(3): 256-269.

Tutak, E., et al. (2009). "A Turkish newborn infant with cerebellar agenesis/neonatal diabetes mellitus and PTF1A mutation." Genet Couns. 20(0): 147-152.

Ubersax, J. A. and J. E. Ferrell, Jr. (2006). "A noisy 'Start' to the cell cycle." Mol Syst Biol 2: 20060014

Uchikawa, M., et al. (1999). "Two distinct subgroups of Group B Sox genes for transcriptional activators and repressors: their expression during embryonic organogenesis of the chicken." Mech Dev 84(1): 103-120.

Uribe, R. A., et al. (2016). "A novel subset of enteric neurons revealed by ptf1a:GFP in the developing zebrafish enteric nervous system." Genesis 54(3): 123-128.

Vernon, A. E. (2003). "The cdk inhibitor p27Xic1 is required for differentiation of primary neurones in Xenopus." Development 130(1): 85-92.

Vosper, J. M., et al. (2007). "Regulation of neurogenin stability by ubiquitinmediated proteolysis." Biochem J 407(2): 277-284. 
Wagner, G., et al. (2017). "Brg1 chromatin remodeling ATPase balances germ layer patterning by amplifying the transcriptional burst at midblastula transition." PLoS Genet 13(5): e1006757.

Wang, F., et al. (2015). "Targeted gene disruption in Xenopus laevis using CRISPR/Cas9." Cell \& bioscience 5(1): 15.

Wapinski, O. L., et al. (2013). "Hierarchical mechanisms for direct reprogramming of fibroblasts to neurons." Cell 155(3): 621-635.

Weedon, M. N., et al. (2014). "Recessive mutations in a distal PTF1A enhancer cause isolated pancreatic agenesis." Nat Genet 46(1): 61-64.

Weintraub, H. (1993). "The MyoD Family and Myogenesis: Redundancy, Networks, and Thresholds." Cell 75(7): 1241-1244.

Wettstein, D. A., et al. (1997). "The Xenopus homolog of Drosophila Suppressor of Hairless mediates Notch signaling during primary neurogenesis." Development 124(3): 693-702.

Wiebe, P. O., et al. (2007). "Ptf1 a binds to and activates area III, a highly conserved region of the $\mathrm{Pdx} 1$ promoter that mediates early pancreas-wide Pdx1 expression." Mol Cell Biol 27(11): 4093-4104.

Wobus, A. M. and K. R. Boheler (2005). "Embryonic stem cells: prospects for developmental biology and cell therapy." Physiological reviews 85(2): 635-678.

Wullimann, M. F., et al. (2005). "Secondary neurogenesis in the brain of the African clawed frog, Xenopus laevis, as revealed by PCNA, Delta-1,

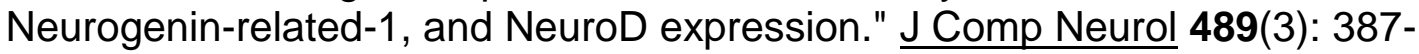
402.

Yamada, M., et al. (2007). "Origin of climbing fiber neurons and their developmental dependence on Ptf1a." J Neurosci 27(41): 10924-10934. Yao, B., et al. (2016). "Epigenetic mechanisms in neurogenesis." Nat Rev Neurosci 17(9): 537-549.

Yao, B. and P. Jin (2014). "Unlocking epigenetic codes in neurogenesis." Genes Dev 28(12): 1253-1271.

Zaret, K. S. and J. S. Carroll (2011). "Pioneer transcription factors: establishing competence for gene expression." Genes Dev 25(21): 22272241.

Zaret, K. S. and S. E. Mango (2016). "Pioneer transcription factors, chromatin dynamics, and cell fate control." Curr Opin Genet Dev 37: 76-81. Zhan, X., et al. (2011). "Dual role of Brg chromatin remodeling factor in Sonic hedgehog signaling during neural development." Proceedings of the National Academy of Sciences 108(31): 12758-12763.

Zhang, Y., et al. (1998). "The dermatomyositis-specific autoantigen Mi2 is a component of a complex containing histone deacetylase and nucleosome remodeling activities." Cell 95(2): 279-289.

Zimmerman, L. B., et al. (1996). "The Spemann Organizer Signal noggin Binds and Inactivates Bone Morphogenetic Protein 4." Cell 86: 599-606. 


\section{Appendix}

\subsection{Summary of Nanostring data}

Given are the average counts after normalization and background subtraction of three independent experiments for each sample and gene, respectively. In a second table, the calculated standard error of the mean (SEM) is given for each sample and gene, respectively. Data were processed as described in Material and Methods.

\subsubsection{Ptf1a threonine mutants}

Table S1: Summary of average normalized counts of three independent Nanostring experiments for each sample and gene

\begin{tabular}{|l|r|r|r|r|r|}
\hline Gene Name & \multicolumn{1}{l|}{ CC } & Ptf1a & Ptf1aW224A/W242A $^{\text {Ptf1a }}{ }^{\text {T243A }}$ & Ptf1a $^{\text {T243E }}$ \\
\hline odc1.L & 15504 & 20639 & 18332 & 17622 & 20778 \\
\hline ppi1.I & 2825 & 3110 & 2921 & 2912 & 3073 \\
\hline rplp0.S & 33879 & 23110 & 27623 & 28940 & 23618 \\
\hline t.S & 81 & 23 & 55 & 47 & 34 \\
\hline pax2.L & 12 & 524 & 11 & 104 & 299 \\
\hline prdm14.L & 17 & 562 & 789 & 889 & 1603 \\
\hline tubb2b.S & 100 & 17477 & 5978 & 9622 & 24822 \\
\hline dll1.L & 14 & 168 & 26 & 49 & 134 \\
\hline ebf2.S & 1 & 493 & 50 & 184 & 476 \\
\hline Ihx1.S & 3 & 779 & 6 & 146 & 904 \\
\hline myt1.S & 430 & 3173 & 1560 & 1697 & 3389 \\
\hline prdm13.S & 4 & 3572 & 33 & 547 & 2303 \\
\hline zc3h12c.L & 432 & 1528 & 1302 & 1017 & 1613 \\
\hline onecut1.2.L & 22 & 9630 & 944 & 4628 & 9215 \\
\hline aldh1b1.L & 9 & 235 & 7 & 31 & 148 \\
\hline barhl2.L & 38 & 238 & 21 & 31 & 169 \\
\hline gad1.1.L & 52 & 2989 & 981 & 2435 \\
\hline kirrel2.L & 11 & 4356 & 406 & 1494 \\
\hline neurog2.S & 3 & 244 & 40 & 129 \\
\hline
\end{tabular}




\begin{tabular}{|l|r|r|r|r|r|}
\hline nr5a2.L & 9 & 737 & 184 & 507 & 1082 \\
\hline tlx3.L & 11 & 70 & 1336 & 492 & 418 \\
\hline pdia2.L & 18 & 62 & 13 & 12 & 35 \\
\hline slc17a7.L & 2 & 219 & 372 & 377 & 648 \\
\hline slc32a1.S & 14 & 583 & 58 & 176 & 773 \\
\hline
\end{tabular}

Table S2: Summary of the calculated SEM of the normalized counts shown in Table S1 for each sample and gene

\begin{tabular}{|c|c|c|c|c|c|}
\hline Gene Name & CC & Ptf1a & Ptf1aW224A/W242A & $\mathrm{Ptf} \mathrm{a}^{\mathrm{T} 243 \mathrm{~A}}$ & Ptf1a $a^{T 243 E}$ \\
\hline odc1.L & 3578 & 4765 & 4568 & 4501 & 4749 \\
\hline ppi1.I & 407 & 479 & 448 & 367 & 307 \\
\hline rplp0.S & 7747 & 5206 & 5543 & 6417 & 6601 \\
\hline t.S & 29 & 8 & 15 & 20 & 16 \\
\hline pax2.L & 3 & 146 & 5 & 18 & 33 \\
\hline prdm14.L & 5 & 159 & 203 & 179 & 80 \\
\hline tubb2b.S & 22 & 3230 & 1981 & 1686 & 2168 \\
\hline dll1.L & 4 & 44 & 13 & 9 & 22 \\
\hline ebf2.S & 1 & 94 & 26 & 33 & 93 \\
\hline $\ln \times 1 . S$ & 1 & 156 & 3 & 31 & 172 \\
\hline myt1.S & 144 & 651 & 625 & 435 & 700 \\
\hline prdm13.S & 2 & 858 & 16 & 111 & 83 \\
\hline zc3h12c.L & 123 & 275 & 401 & 195 & 324 \\
\hline onecut1.2.L & 5 & 1484 & 325 & 797 & 1383 \\
\hline aldh1b1.L & 2 & 54 & 3 & 9 & 14 \\
\hline barhl2.L & 13 & 38 & 7 & 10 & 10 \\
\hline gad1.1.L & 33 & 483 & 8 & 99 & 259 \\
\hline kirrel2.L & 2 & 1082 & 8 & 88 & 217 \\
\hline neurog2.S & 1 & 85 & 3 & 20 & 33 \\
\hline nr5a2.L & 3 & 117 & 61 & 134 & 229 \\
\hline tlx3.L & 7 & 13 & 400 & 101 & 159 \\
\hline pdia2.L & 9 & 5 & 0 & 3 & 4 \\
\hline
\end{tabular}




\begin{tabular}{|l|r|r|r|r|r|}
\hline slc17a7.L & 1 & 75 & 93 & 82 & 77 \\
\hline slc32a1.S & 4 & 100 & 19 & 50 & 218 \\
\hline
\end{tabular}

\subsubsection{Time course analysis}

Table S3: Summary of average normalized counts of three independent Nanostring experiments for each sample and gene

\begin{tabular}{|c|c|c|c|c|c|c|c|c|c|c|c|c|}
\hline & \multicolumn{2}{|c|}{$3 \mathrm{~h}$} & \multicolumn{2}{|c|}{$6 \mathrm{~h}$} & \multicolumn{2}{|c|}{$9 \mathrm{~h}$} & \multicolumn{2}{|c|}{$12 \mathrm{~h}$} & \multicolumn{2}{|c|}{$15 \mathrm{~h}$} & \multicolumn{2}{|c|}{$25 \mathrm{~h}$} \\
\hline Gene name & CC & Ptf1a & CC & Ptf1a & CC & Ptf1a & CC & Ptf1a & CC & Ptf1a & CC & Ptf1a \\
\hline odc1.L & 33893 & 33078 & 40930 & 42484 & 43118 & 41525 & 31637 & 35136 & 26616 & 29026 & 13869 & 14788 \\
\hline ppi1.I & 2092 & 2094 & 2092 & 2094 & 2090 & 2093 & 2093 & 2090 & 2091 & 2088 & 2090 & 2086 \\
\hline rplp0.S & 8787 & 8936 & 8803 & 9148 & 8850 & 9127 & 9872 & 10422 & 11934 & 10118 & 28671 & 20126 \\
\hline$t . S$ & 38 & 169 & 67 & 58 & 57 & 67 & 81 & 45 & 41 & 31 & 140 & 17 \\
\hline pax2.L & 4 & 8 & 5 & 16 & 4 & 24 & 9 & 116 & 11 & 255 & 28 & 403 \\
\hline prdm14.L & 392 & 705 & 536 & 794 & 349 & 377 & 265 & 365 & 212 & 308 & 7 & 101 \\
\hline tubb2b.S & 36 & 46 & 59 & 126 & 69 & 147 & 66 & 348 & 48 & 958 & 59 & 7236 \\
\hline dll1.L & 61 & 264 & 127 & 672 & 214 & 841 & 72 & 537 & 36 & 530 & 16 & 181 \\
\hline$e b f 2 . S$ & 1 & 151 & 1 & 682 & 1 & 451 & 1 & 342 & 1 & 424 & 1 & 234 \\
\hline $\ln \times 1 . S$ & 56 & 77 & 37 & 99 & 2 & 82 & 1 & 93 & 1 & 139 & 1 & 305 \\
\hline myt1.S & 149 & 1512 & 279 & 9508 & 590 & 10047 & 1099 & 7541 & 1400 & 6967 & 358 & 2421 \\
\hline prdm13.S & 1 & 348 & 1 & 2845 & 1 & 4498 & 1 & 4960 & 1 & 4970 & 1 & 2172 \\
\hline zc3h12c.L & 118 & 1845 & 270 & 6817 & 351 & 5820 & 157 & 3947 & 123 & 3283 & 528 & 1038 \\
\hline onecut1.2.L & 21 & 18 & 101 & 422 & 508 & 1966 & 777 & 5344 & 544 & 6221 & 10 & 5517 \\
\hline aldh1b1.L & 2 & 47 & 2 & 169 & 4 & 523 & 1 & 824 & 1 & 786 & 3 & 159 \\
\hline barhl2.L & 18 & 37 & 15 & 115 & 22 & 476 & 14 & 974 & 9 & 686 & 9 & 118 \\
\hline gad1.1.L & 1 & 1 & 1 & 1 & 1 & 14 & 1 & 385 & 1 & 697 & 72 & 1027 \\
\hline kirrel2.L & 1028 & 938 & 1251 & 2695 & 761 & 5455 & 71 & 6693 & 26 & 5610 & 13 & 3474 \\
\hline neurog2.S & 182 & 235 & 104 & 120 & 49 & 171 & 20 & 641 & 17 & 667 & 2 & 252 \\
\hline nr5a2.L & 2 & 12 & 2 & 146 & 7 & 330 & 83 & 543 & 65 & 538 & 1 & 365 \\
\hline$t / x 3 . L$ & 27 & 24 & 31 & 125 & 24 & 326 & 11 & 266 & 23 & 211 & 21 & 11 \\
\hline pdia2.L & 2 & 19 & 1 & 36 & 1 & 110 & 1 & 162 & 1 & 160 & 12 & 74 \\
\hline slc17a7.L & 3 & 2 & 1 & 1 & 1 & 2 & 2 & 1 & 1 & 1 & 1 & 17 \\
\hline slc32a1.S & 21 & 21 & 16 & 21 & 7 & 15 & 2 & 44 & 2 & 39 & 2 & 248 \\
\hline
\end{tabular}


Table S4: Summary of the calculated SEM of the normalized counts shown in Table S3 for each sample and gene

\begin{tabular}{|c|c|c|c|c|c|c|c|c|c|c|c|c|}
\hline & \multicolumn{2}{|c|}{$3 \mathrm{~h}$} & \multicolumn{2}{|c|}{$6 \mathrm{~h}$} & \multicolumn{2}{|c|}{$9 \mathrm{~h}$} & \multicolumn{2}{|c|}{$12 \mathrm{~h}$} & \multicolumn{2}{|c|}{$15 \mathrm{~h}$} & \multicolumn{2}{|c|}{$25 \mathrm{~h}$} \\
\hline Gene Name & CC & Ptf1a & CC & Ptf1a & CC & Ptf1a & CC & Ptf1a & CC & Ptf1a & CC & Ptf1a \\
\hline odc1.L & 2289 & 3005 & 4585 & 5388 & 5548 & 3565 & 4115 & 2821 & 3236 & 2935 & 1943 & 2463 \\
\hline ppi1.l & 37 & 36 & 36 & 37 & 36 & 37 & 37 & 37 & 37 & 39 & 36 & 36 \\
\hline rplp0.S & 817 & 885 & 956 & 1333 & 496 & 351 & 794 & 687 & 1016 & 772 & 2715 & 2286 \\
\hline t.S & 4 & 56 & 32 & 10 & 3 & 35 & 41 & 22 & 17 & 5 & 23 & 3 \\
\hline pax2.L & 2 & 2 & 1 & 6 & 2 & 3 & 2 & 16 & 5 & 27 & 13 & 29 \\
\hline prdm14.L & 93 & 113 & 58 & 223 & 90 & 57 & 188 & 58 & 157 & 105 & 4 & 17 \\
\hline tubb2b.S & 7 & 8 & 10 & 26 & 16 & 29 & 10 & 46 & 7 & 43 & 7 & 237 \\
\hline dIII.L & 30 & 24 & 37 & 70 & 71 & 134 & 17 & 108 & 11 & 98 & 3 & 22 \\
\hline$e b f 2 . S$ & 0 & 24 & 0 & 35 & 0 & 54 & 0 & 36 & 0 & 20 & 0 & 15 \\
\hline $\operatorname{lh} \times 1 . S$ & 6 & 4 & 12 & 7 & 1 & 5 & 0 & 17 & 0 & 12 & 0 & 23 \\
\hline myt1.S & 37 & 120 & 63 & 645 & 171 & 1555 & 138 & 1048 & 272 & 915 & 51 & 180 \\
\hline prdm13.S & 0 & 62 & 0 & 323 & 0 & 866 & 0 & 667 & 0 & 527 & 0 & 176 \\
\hline zc3h12c.L & 59 & 286 & 86 & 321 & 106 & 226 & 65 & 380 & 54 & 277 & 97 & 110 \\
\hline onecut1.2.L & 14 & 5 & 35 & 116 & 203 & 830 & 126 & 766 & 60 & 617 & 2 & 349 \\
\hline aldh1b1.L & 1 & 20 & 1 & 65 & 1 & 154 & 0 & 306 & 0 & 245 & 1 & 38 \\
\hline barh/2.L & 4 & 12 & 5 & 23 & 8 & 194 & 3 & 236 & 3 & 123 & 3 & 12 \\
\hline gad1.1.L & 0 & 0 & 0 & 0 & 0 & 0 & 0 & 70 & 0 & 107 & 53 & 134 \\
\hline kirrel2.L & 147 & 130 & 150 & 427 & 90 & 1471 & 17 & 797 & 4 & 582 & 6 & 213 \\
\hline neurog2.S & 7 & 69 & 13 & 14 & 9 & 42 & 11 & 83 & 11 & 72 & 1 & 30 \\
\hline$n r 5 a 2 . L$ & 1 & 4 & 1 & 40 & 2 & 103 & 11 & 94 & 15 & 71 & 0 & 46 \\
\hline$t / x 3 . L$ & 7 & 4 & 9 & 16 & 2 & 97 & 5 & 50 & 18 & 56 & 11 & 4 \\
\hline pdia2.L & 1 & 3 & 0 & 11 & 0 & 19 & 0 & 30 & 0 & 14 & 5 & 7 \\
\hline slc17a7.L & 1 & 1 & 0 & 0 & 0 & 1 & 0 & 0 & 0 & 0 & 0 & 4 \\
\hline slc32a1.S & 3 & 8 & 4 & 4 & 3 & 3 & 1 & 10 & 1 & 12 & 1 & 56 \\
\hline
\end{tabular}

\subsubsection{Brg1 knock-down}

Table S5: Summary of average normalized counts of three independent Nanostring experiments for each sample and gene

\begin{tabular}{|l|c|c|c|c|}
\hline Gene Name & CC & Ptf1a & Ptf1a + cMO & Ptf1a + Brg1MO \\
\hline LOC100487395.L & 0 & 0 & 3 & 0 \\
\hline
\end{tabular}




\begin{tabular}{|c|c|c|c|c|}
\hline Xelaev18005831m.g & 20 & 247 & 280 & 177 \\
\hline Xelaev18007508m.g & 1 & 2650 & 2653 & 857 \\
\hline Xelaev18040877m.g & 12 & 877 & 1020 & 386 \\
\hline Xetrov90021170m.L & 43 & 45 & 37 & 77 \\
\hline adamts20.L & 1 & 0 & 4 & 5 \\
\hline aldh1b1.L & 1 & 38 & 59 & 24 \\
\hline arid1b.L & 71 & 1961 & 2065 & 1368 \\
\hline arl4a.S & 7406 & 8582 & 8358 & 11119 \\
\hline b4galnt1.S & 9 & 300 & 260 & 86 \\
\hline barhl2.L & 6 & 484 & 619 & 187 \\
\hline bgn.L & 2 & 771 & 585 & 195 \\
\hline bhlhe40.L & 53 & 244 & 233 & 115 \\
\hline cacna2d2.L & 2 & 69 & 79 & 32 \\
\hline cbfa2t2.S & 360 & 7883 & 8720 & 9569 \\
\hline chst3.L & 1 & 641 & 762 & 466 \\
\hline dll1.L & 52 & 395 & 403 & 402 \\
\hline$d m b \times 1 . S$ & 12 & 71 & 109 & 54 \\
\hline dmrta2.L & 6 & 576 & 628 & 141 \\
\hline dpys/3.L & 7 & 291 & 311 & 82 \\
\hline $\operatorname{drg} x . L$ & 21 & 161 & 177 & 59 \\
\hline ebf2.S & 1 & 371 & 435 & 261 \\
\hline efnb1.L & 17 & 19 & 22 & 25 \\
\hline elavl3.L & 298 & 9329 & 11480 & 7174 \\
\hline eya1.L & 882 & 6855 & 7133 & 4627 \\
\hline fam102b.L & 85 & 48 & 47 & 96 \\
\hline$f z d 10 . S$ & 1513 & 1227 & 1248 & 1785 \\
\hline gad1.1.L & 1 & 1293 & 764 & 133 \\
\hline gas6.L & 1146 & 2812 & 2280 & 2107 \\
\hline gfi1.L & 56 & 345 & 405 & 653 \\
\hline gfra1.S & 56 & 1575 & 1800 & 907 \\
\hline hes3.3.S & 1752 & 3874 & 4086 & 4770 \\
\hline hes5.1.S & 819 & 3827 & 4457 & 4893 \\
\hline hes5.2.S & 0 & 132 & 143 & 25 \\
\hline hes9-1.S & 54 & 524 & 547 & 243 \\
\hline hesx1.S & 21 & 3694 & 3490 & 740 \\
\hline hoxc4.S & 3 & 24 & 30 & 22 \\
\hline hoxd1.L & 16 & 103 & 95 & 299 \\
\hline id2.L & 11345 & 20374 & 19866 & 25793 \\
\hline
\end{tabular}




\begin{tabular}{|c|c|c|c|c|}
\hline id4.S & 3645 & 7030 & 6969 & 5193 \\
\hline insm1.L & 258 & 3388 & 3753 & 2799 \\
\hline kif26a.L & 0 & 31 & 49 & 5 \\
\hline kirrel2.L & 30 & 3798 & 4499 & 3343 \\
\hline k/h/14.L & 0 & 0 & 1 & 0 \\
\hline Ibx $\times 1 . S$ & 1 & 92 & 122 & 24 \\
\hline $\ln \times 1 . S$ & 2 & 89 & 126 & 23 \\
\hline map2.S & 6 & 709 & 741 & 307 \\
\hline map3k12.S & 13 & 120 & 152 & 48 \\
\hline$m m p 28 . L$ & 30 & 653 & 648 & 102 \\
\hline mxra7.L & 33 & 1324 & 1579 & 755 \\
\hline myt1.S & 1589 & 7720 & 8590 & 6062 \\
\hline ncam1.S & 76 & 822 & 880 & 159 \\
\hline nes.L & 22 & 1019 & 1488 & 651 \\
\hline neurod4.L & 2 & 5382 & 5844 & 1307 \\
\hline neurog2.S & 10 & 277 & 373 & 428 \\
\hline nr5a2.L & 6 & 29 & 37 & 4 \\
\hline olig2.S & 30 & 1645 & 2079 & 1253 \\
\hline onecut1.2.L & 661 & 4637 & 4972 & 1747 \\
\hline otx2.L & 367 & 776 & 1116 & 663 \\
\hline pax2.L & 2 & 55 & 69 & 33 \\
\hline pcdh18.L & 1 & 3 & 9 & 25 \\
\hline pck1.L & 27 & 930 & 715 & 142 \\
\hline pdia2.L & 1 & 37 & 37 & 38 \\
\hline plk3.L & 177 & 1473 & 1625 & 960 \\
\hline pou3f1.S & 2 & 1510 & 2069 & 1137 \\
\hline pou3f2.L & 37 & 494 & 680 & 238 \\
\hline ppp1r9b.S & 4 & 418 & 428 & 53 \\
\hline prdm13.S & 1 & 4303 & 4560 & 2242 \\
\hline prdm14.L & 337 & 434 & 698 & 680 \\
\hline prph.S & 10 & 2106 & 2578 & 352 \\
\hline prr15l.S & 0 & 1 & 1 & 2 \\
\hline rapgef5.L & 1149 & 812 & 690 & 565 \\
\hline runx1t1.L & 12 & 482 & 489 & 181 \\
\hline scrt1.S & 1 & 1523 & 1930 & 489 \\
\hline six3.L & 75 & 1025 & 1181 & 183 \\
\hline slc17a7.L & 2 & 0 & 4 & 4 \\
\hline slc18a3.S & 9 & 843 & 924 & 751 \\
\hline
\end{tabular}




\begin{tabular}{|c|c|c|c|c|}
\hline slc32a1.S & 2 & 6 & 11 & 2 \\
\hline slc3a2.L & 45 & 2599 & 2543 & 170 \\
\hline slc43a2.S & 29 & 692 & 841 & 351 \\
\hline snai2.L & 85 & 885 & 1121 & 1004 \\
\hline snap25.L & 14 & 1227 & 992 & 377 \\
\hline snrk.S & 179 & 190 & 189 & 405 \\
\hline Sox11.L & 906 & 9292 & 12047 & 6872 \\
\hline Sox9.L & 15 & 385 & 420 & 166 \\
\hline t.S & 7 & 8 & 12 & 20 \\
\hline tfap2b.S & 21 & 2824 & 2672 & 999 \\
\hline$t / \times 3 . L$ & 22 & 434 & 526 & 428 \\
\hline tox3.L & 543 & 2779 & 3087 & 2363 \\
\hline tubb2b.S & 94 & 369 & 494 & 176 \\
\hline tubb3.L & 1 & 130 & 221 & 24 \\
\hline vcan.L & 9 & 58 & 80 & 86 \\
\hline zc3h12c.L & 222 & 3702 & 4360 & 3906 \\
\hline odc1.L & 25972 & 27916 & 29603 & 24441 \\
\hline ppi1.I & 2249 & 2365 & 2324 & 2117 \\
\hline rplp0.S & 6807 & 5917 & 5697 & 7544 \\
\hline
\end{tabular}

TableS6: Summary of the calculated SEM of the normalized counts shown in TableS5 for each sample and gene

\begin{tabular}{|l|c|c|c|c|}
\hline Gene Name & CC & Ptf1a & Ptf1a + cMO & Ptf1a + Brg1MO \\
\hline LOC100487395.L & 0.3 & 0.3 & 1.4 & 0.3 \\
\hline Xelaev18005831m.g & 12.0 & 91.9 & 73.6 & 55.1 \\
\hline Xelaev18007508m.g & 0.4 & 266.0 & 669.2 & 53.5 \\
\hline Xelaev18040877m.g & 4.0 & 125.1 & 72.8 & 154.7 \\
\hline Xetrov90021170m.L & 11.4 & 2.8 & 8.7 & 22.0 \\
\hline adamts20.L & 1.2 & 0.3 & 1.8 & 1.4 \\
\hline aldh1b1.L & 0.4 & 11.9 & 28.5 & 4.2 \\
\hline arid1b.L & 6.6 & 309.4 & 337.5 & 162.3 \\
\hline arl4a.S & 1032.3 & 1162.4 & 853.1 & 1197.2 \\
\hline b4galnt1.S & 4.8 & 86.9 & 72.1 & 23.6 \\
\hline barhl2.L & 2.5 & 67.4 & 105.4 & 77.3 \\
\hline bgn.L & 1.9 & 290.7 & 129.7 & 60.9 \\
\hline bhlhe40.L & 6.5 & 39.2 & 26.4 & 11.8 \\
\hline
\end{tabular}




\begin{tabular}{|c|c|c|c|c|}
\hline cacna2d2.L & 0.8 & 18.6 & 17.9 & 11.0 \\
\hline cbfa2t2.S & 122.8 & 1600.8 & 1785.0 & 1799.8 \\
\hline chst3.L & 1.2 & 120.1 & 146.5 & 92.7 \\
\hline$d / I 1 . L$ & 16.8 & 66.4 & 106.8 & 160.9 \\
\hline$d m b \times 1 . S$ & 8.3 & 4.2 & 22.9 & 16.1 \\
\hline dmrta2.L & 4.8 & 145.0 & 186.9 & 58.3 \\
\hline dpys/3.L & 2.5 & 79.4 & 84.2 & 27.8 \\
\hline $\operatorname{drgx} . L$ & 4.7 & 35.7 & 32.2 & 1.9 \\
\hline$e b f 2 . S$ & 0.4 & 47.0 & 94.9 & 38.3 \\
\hline efnb1.L & 6.3 & 5.0 & 0.6 & 2.5 \\
\hline elav/3.L & 20.9 & 1184.4 & 1339.5 & 1441.1 \\
\hline eya1.L & 297.7 & 1517.1 & 1473.2 & 1064.7 \\
\hline fam102b.L & 10.2 & 13.1 & 7.0 & 24.5 \\
\hline$f z d 10 . S$ & 283.5 & 294.1 & 330.8 & 321.9 \\
\hline gad1.1.L & 0.4 & 536.2 & 101.1 & 78.2 \\
\hline gas6.L & 442.4 & 971.7 & 474.5 & 392.7 \\
\hline gfi1.L & 11.6 & 74.3 & 67.4 & 190.4 \\
\hline gfra1.S & 11.5 & 77.3 & 58.4 & 235.7 \\
\hline hes3.3.S & 561.9 & 1095.9 & 1239.8 & 1551.3 \\
\hline hes5.1.S & 187.4 & 554.1 & 541.7 & 1153.2 \\
\hline hes5.2.S & 0.3 & 33.1 & 15.6 & 6.9 \\
\hline hes9-1.S & 11.2 & 71.5 & 51.3 & 46.0 \\
\hline hesx1.S & 10.9 & 797.8 & 641.8 & 197.8 \\
\hline hoxc4.S & 1.7 & 7.4 & 6.0 & 6.5 \\
\hline hoxd1.L & 6.9 & 28.7 & 40.9 & 81.6 \\
\hline$i d 2 . L$ & 3042.5 & 6611.5 & 5984.5 & 8745.8 \\
\hline id4.S & 835.0 & 1936.9 & 1406.9 & 1060.0 \\
\hline insm1.L & 101.7 & 844.6 & 914.4 & 276.8 \\
\hline kif26a.L & 0.3 & 13.7 & 14.7 & 3.5 \\
\hline kirrel2.L & 7.6 & 226.6 & 587.5 & 105.0 \\
\hline k/h/14.L & 0.3 & 0.3 & 0.4 & 0.3 \\
\hline$I b \times 1 . S$ & 0.4 & 11.5 & 21.8 & 12.4 \\
\hline $\operatorname{lh} x 1 . S$ & 0.7 & 37.5 & 20.9 & 6.3 \\
\hline map2.S & 0.9 & 132.5 & 130.9 & 46.9 \\
\hline map3k12.S & 5.7 & 33.3 & 5.8 & 14.9 \\
\hline$m m p 28 . L$ & 11.0 & 199.2 & 179.1 & 17.9 \\
\hline mxra7.L & 10.2 & 380.8 & 369.8 & 134.4 \\
\hline myt1.S & 726.4 & 1958.6 & 2241.6 & 1004.7 \\
\hline
\end{tabular}




\begin{tabular}{|c|c|c|c|c|}
\hline ncam1.S & 6.9 & 131.2 & 120.8 & 34.5 \\
\hline nes.L & 2.0 & 70.8 & 106.6 & 128.9 \\
\hline neurod4.L & 1.2 & 760.6 & 1314.2 & 120.2 \\
\hline neurog2.S & 4.0 & 50.5 & 39.7 & 26.2 \\
\hline nr5a2.L & 2.1 & 8.4 & 8.2 & 3.2 \\
\hline olig2.S & 8.9 & 518.4 & 542.8 & 63.8 \\
\hline onecut1.2.L & 191.8 & 1040.7 & 672.5 & 436.2 \\
\hline otx2.L & 69.5 & 14.6 & 74.1 & 224.8 \\
\hline pax2.L & 0.5 & 8.5 & 12.0 & 14.7 \\
\hline pcdh18.L & 0.1 & 1.0 & 0.5 & 3.7 \\
\hline pck1.L & 15.8 & 222.2 & 177.1 & 27.5 \\
\hline pdia2.L & 0.4 & 7.0 & 9.7 & 11.7 \\
\hline plk3.L & 38.6 & 118.7 & 165.0 & 81.0 \\
\hline pou3f1.S & 1.9 & 480.6 & 740.8 & 304.0 \\
\hline pou3f2.L & 11.0 & 96.0 & 189.0 & 42.7 \\
\hline ppp1rgb.S & 3.3 & 69.0 & 27.5 & 31.6 \\
\hline prdm13.S & 0.2 & 531.2 & 705.8 & 272.9 \\
\hline prdm14.L & 128.7 & 27.3 & 86.6 & 147.4 \\
\hline prph.S & 3.0 & 345.1 & 393.2 & 154.0 \\
\hline prr15l.S & 0.3 & 0.4 & 0.4 & 1.6 \\
\hline rapgef5.L & 332.6 & 220.2 & 131.5 & 89.0 \\
\hline runx1t1.L & 5.8 & 82.0 & 90.8 & 7.4 \\
\hline scrt1.S & 0.4 & 122.9 & 340.8 & 115.4 \\
\hline six3.L & 29.2 & 134.9 & 143.9 & 53.6 \\
\hline slc17a7.L & 1.3 & 0.2 & 1.6 & 2.1 \\
\hline slc18a3.S & 5.9 & 235.1 & 255.6 & 129.1 \\
\hline slc32a1.S & 1.1 & 1.4 & 1.6 & 0.6 \\
\hline slc3a2.L & 10.9 & 640.0 & 571.7 & 86.7 \\
\hline slc43a2.S & 3.9 & 134.9 & 91.4 & 63.4 \\
\hline snai2.L & 30.1 & 126.7 & 170.1 & 228.2 \\
\hline snap25.L & 6.2 & 232.0 & 121.8 & 55.4 \\
\hline snrk.S & 21.9 & 11.9 & 16.9 & 100.3 \\
\hline SOX11.L & 302.1 & 734.5 & 1214.8 & 729.2 \\
\hline Sox9.L & 3.5 & 25.1 & 33.0 & 41.7 \\
\hline$t . S$ & 5.7 & 0.7 & 2.1 & 7.4 \\
\hline tfap2b.S & 1.1 & 179.5 & 247.8 & 347.4 \\
\hline$t / x 3 . L$ & 12.3 & 141.6 & 149.1 & 109.3 \\
\hline tox3.L & 189.7 & 534.0 & 504.7 & 419.6 \\
\hline
\end{tabular}




\begin{tabular}{|l|c|c|c|c|}
\hline tubb2b.S & 5.9 & 57.4 & 37.5 & 20.8 \\
\hline tubb3.L & 0.1 & 14.1 & 14.5 & 10.3 \\
\hline vcan.L & 3.0 & 6.2 & 8.8 & 15.3 \\
\hline zc3h12c.L & 107.2 & 944.4 & 1209.4 & 1032.3 \\
\hline odc1.L & 5393.4 & 5003.5 & 5961.8 & 4832.0 \\
\hline ppi1.I & 183.5 & 231.2 & 273.2 & 243.0 \\
\hline rplp0.S & 1306.4 & 1089.4 & 825.9 & 1179.1 \\
\hline
\end{tabular}

\subsection{Summary of the genes analyzed with the Nanostring}

Table S7: Summary of the geneses analyzed with Nanostring. Given is the gene symbol, its accession number as well as the target region and the target sequence. Grey marks the genes analyzed on the 24 gene codeset used for analysis of the time course and threonine mutants experiments

\begin{tabular}{|c|c|c|c|}
\hline Gene & Accession & Target region & Target Sequence \\
\hline odc1.L & $\begin{array}{l}\text { NM_001086 } \\
698.1\end{array}$ & $856-955$ & $\begin{array}{l}\text { GGATATAATTGGTGTGAGTTTCCATGTTGGCAGTGGCTGC } \\
\text { ACTGATCCACAGACTTATGTACAAGCTGTCTCAGATGCAC } \\
\text { GATGTGTCTTTGACATGGGG }\end{array}$ \\
\hline ppi1.l & $\begin{array}{l}\text { NM_001193 } \\
402.1\end{array}$ & $430-529$ & $\begin{array}{l}\text { AAACAGACTTGGAATGGTGGAAACAGATTCTCAGGACCG } \\
\text { ACCACTTGATGAAGTTCAAGTATTGCGGGCTTATCCATCT } \\
\text { GGCTAAATGGAGGCAGATAAA }\end{array}$ \\
\hline rplpo.s & $\begin{array}{l}\text { NM_001086 } \\
665.1\end{array}$ & $127-226$ & $\begin{array}{l}\text { TCAATTGCTCGATGATTATCCAAAATGCTTCATTGTGGGG } \\
\text { GCGGACAATGTTGGTTCAAAACAAATGCAGCAGATCCGTA } \\
\text { TGTCCCTGCGTGGAAAAGCT }\end{array}$ \\
\hline$t . S$ & $\begin{array}{l}\text { NM_001090 } \\
578.1\end{array}$ & $1530-1629$ & $\begin{array}{l}\text { TGTAGGCCTCCAAAACAACTTAAAGATGTGCTTAGGCAAG } \\
\text { TTATATCAGTGTTTACCTGCTTCTAAAGACTTCATGGGCCC } \\
\text { AACCAGGTGTGGGTGGTCT }\end{array}$ \\
\hline tubb2b.S & $\begin{array}{l}\text { NM_001086 } \\
064.1\end{array}$ & $901-1000$ & $\begin{array}{l}\text { TGCACTTTTTTATGCCAGGCTTTGCCCCATTAACAAGTCG } \\
\text { TGGCAGCCAACAATACCGAGCCCTGACAGTGCCAGAACT } \\
\text { AACACAGCAAATGTTTGATTC }\end{array}$ \\
\hline neurog2.S & $\begin{array}{l}\text { NM_001088 } \\
335.1\end{array}$ & $1173-1272$ & $\begin{array}{l}\text { CATCGTTAGCTATGTGTATTAGGAAACTGTCTATCCCTCAT } \\
\text { CTGCACCTGTTAGACTACAGCTACCAACTTCCTGTTACCA } \\
\text { GGGGGCTACTGGGTAATGT }\end{array}$ \\
\hline ebf2.S & $\begin{array}{l}\text { NM_001085 } \\
678.1\end{array}$ & $1765-1864$ & $\begin{array}{l}\text { CTCCAATGGTTTCCGAGAAAATCTGTTGAAGGACAGTCCT } \\
\text { GCCATGAGGCAAGGTCAATACTTCGCCCAGTGCAGCAAT } \\
\text { CCCTCAGTGGGTTTCTGGTAG }\end{array}$ \\
\hline$t \mid x 3 . L$ & $\begin{array}{l}\text { XM_018252 } \\
115.1\end{array}$ & $340-439$ & $\begin{array}{l}\text { ACCTTCATGGATGTGCTGTGAGGAAGACGAAGCGTTTAG } \\
\text { AGGGGAGAGTTCTCATTTGAGACAATAAATCTATCGACGG } \\
\text { AGGATGGATCAGCCAACAACT }\end{array}$ \\
\hline $\operatorname{gad1.1.L}$ & $\begin{array}{l}\text { NM_001085 } \\
801.1\end{array}$ & $795-894$ & $\begin{array}{l}\text { GGCAGCAGTCCCCAGATTGGTCCTGTTTACTTCAGAACAC } \\
\text { AGCCACTATTCCATAAAGAAAACTGGCGCGGCATTAGGAT } \\
\text { TTGGAAGTGAAAATGTGATC }\end{array}$ \\
\hline slc32a1.S & $\begin{array}{l}\text { NM_001086 } \\
492.1\end{array}$ & $1078-1177$ & $\begin{array}{l}\text { TTAATGTACAACAGCTTTCCAAACCTGCCCATATCCCAGA } \\
\text { AGTCTTGGTCCATCATGGCCACTGCTGTGCTCCTGCCCT } \\
\text { GTGCATTTCTCAAGAACCTTA }\end{array}$ \\
\hline
\end{tabular}




\begin{tabular}{|c|c|c|c|}
\hline slc17a7.L & $\begin{array}{l}\text { NM_001089 } \\
635.1\end{array}$ & 2178-2277 & $\begin{array}{l}\text { AGTGAATTGAGTTAGATGTACACATTACTGCGTGATTCCC } \\
\text { AGGGATACGGTGTAGAAAATATCCATGAGGATGGGGCAT } \\
\text { GATCAATTCTACATCACTTGG }\end{array}$ \\
\hline prdm14.L & $\begin{array}{l}\text { Xelaev1803 } \\
2099 \mathrm{~m} .1\end{array}$ & $586-685$ & $\begin{array}{l}\text { ATCTCCCATAGTCCCCAAAAAGTGCAAAAATGTGTCCCTG } \\
\text { AGTGTCCCCAGCCTACAGGTGAGACCCACTCGCACTTAC } \\
\text { CACTTTACAGAAGAAGACTTA }\end{array}$ \\
\hline prdm13.S & $\begin{array}{l}\text { XM_018265 } \\
418.1\end{array}$ & 33-132 & $\begin{array}{l}\text { ATTGGATTTTTAGGAATGAGAAGGTCGCCTGGACCATTGT } \\
\text { CTGGTTACCATTCATGGGAGCTGATTATGCAGGAGGCTTA } \\
\text { GGGAAAGTCTCCATGTGACC }\end{array}$ \\
\hline zc3h12c.L & $\begin{array}{l}\text { XM_018247 } \\
762.1\end{array}$ & $86-185$ & $\begin{array}{l}\text { GTGTCTGAGGCGGGCAGCTTGCTCCCTAATGACTTGGCC } \\
\text { TCTCACTGAGCAGCTGCCTGCTGCCTCCTTTGTGTTGGCC } \\
\text { TATTGTTGCCGCACACCCCTC }\end{array}$ \\
\hline myt1.S & $\begin{array}{l}\text { NM_001088 } \\
192.1\end{array}$ & $685-784$ & $\begin{array}{l}\text { GAGCTAAACAATGAAAAGCCAACTTCAGTAAAGTCGGGTC } \\
\text { AAGCGGAAATAGAACAACTTATGGTAGAAGAGGCGTGTG } \\
\text { AGAAAGAAATCATCATCCAGA }\end{array}$ \\
\hline dII1.L & $\begin{array}{l}\text { XM_018263 } \\
063.1\end{array}$ & $3628-3727$ & $\begin{array}{l}\text { CCCATGTAACTTCAACAGTATTTCTGGTGGGGGTTCACTG } \\
\text { TGATTGGCAGAGGAGTGGGTGTAGTATCTCTGGCGTCAA } \\
\text { CCCTGCATGCTTTTGGTCCCT }\end{array}$ \\
\hline kirrel2.L & $\begin{array}{l}\text { NM_001086 } \\
488.1\end{array}$ & $691-790$ & $\begin{array}{l}\text { CCCAGTCGCCAATGTCCTGCTTGATGTACCCTACAATCTC } \\
\text { ACCTGTCTTGCTTCCGTCGCTAAGCCTGCTGCAGAGATTA } \\
\text { CCTGGTTCCGTGATGGAAAG }\end{array}$ \\
\hline pax2.L & $\begin{array}{l}\text { NM_001086 } \\
361.1\end{array}$ & $1495-1594$ & $\begin{array}{l}\text { GAGTGGCCTACTCTGCTTTCCCTACTGACGTTTCTCAGCT } \\
\text { GTAATAAGCTGCTCTTACCTCTGCTGCAAGCCTCTTTGTT } \\
\text { GTTATTGTGCACGGTAGCTA }\end{array}$ \\
\hline Ihx1.S & $\begin{array}{l}\text { NM_001090 } \\
659.1\end{array}$ & $22-121$ & $\begin{array}{l}\text { TCTATTCTCCTAATCCGCCCATTCCTCTAAAATCCCAAATA } \\
\text { ACCAAAGGCAATGGTTCACTGTGCTGGATGCGAAAGGCC } \\
\text { CATTCTGGACCGTTTCTTGT }\end{array}$ \\
\hline onecut1.2.L & $\begin{array}{l}\text { XM_002935 } \\
458.4\end{array}$ & $4346-4445$ & $\begin{array}{l}\text { ATGCTCATTATAACAAGGAGAGTCTGGGGTGAGGTCCCC } \\
\text { AATTTGTGTGGGGGATTCAACTGAGGAACCACAGGAAAT } \\
\text { GCAGGCAAGAAGGGATCTTTTC }\end{array}$ \\
\hline barh/2.L & $\begin{array}{l}\text { NM_001161 } \\
386.1\end{array}$ & $860-959$ & $\begin{array}{l}\text { ATTGGAGTTACTAGCCGAAGCTGGCAATTATTCAGCCCTG } \\
\text { CAGAGAATGTTCCCATCACCCTATTTCTATCATCCGAGTC } \\
\text { TGCTGAGCAGTATGGACAGT }\end{array}$ \\
\hline nr5a2.L & $\begin{array}{l}\text { NM_001087 } \\
716.1\end{array}$ & $152-251$ & $\begin{array}{l}\text { CCACTTACTCCCTAGCTCATGCTCCCTACGGAACTTGCTT } \\
\text { TATCCGATGACTGTAATTCCAATTCTGCCACAGGACAACC } \\
\text { GACTGATTCACCTCCAAATC }\end{array}$ \\
\hline aldh1b1.L & $\begin{array}{l}\text { XM_018257 } \\
027.1\end{array}$ & $967-1066$ & $\begin{array}{l}\text { AGAACAAGCCGTGGAACAATGTCACGAGGCTTTGTTTTTT } \\
\text { AACATGGGACAGTGTTGTGCTGCAGGTTCCAGGACTTTTG } \\
\text { TGGAGGAAACGATCTACCGT }\end{array}$ \\
\hline pdia2.L & $\begin{array}{l}\text { NM_001090 } \\
179.1\end{array}$ & $882-981$ & $\begin{array}{l}\text { TGCTGCCCAGATCCCAAACCACTTGTTGCTGTTTATCAAT } \\
\text { AAGAGTGACGATTCCCAACTGGTGCTGCTGGAACATTTCC } \\
\text { GCAAAGCAGCTCCTGACTTT }\end{array}$ \\
\hline arl4a.S & $\begin{array}{l}\text { NM_001090 } \\
805.1\end{array}$ & 893-992 & $\begin{array}{l}\text { AAACACCAGGAAACCTAATGTACGTCAGAGGCTGAAACG } \\
\text { CAATCTAGTCATTGTGAGTGTAAACTGTGAATGTGTGCAG } \\
\text { GGTGTTGAAACAGCTGAGGGT }\end{array}$ \\
\hline elav/3.L & $\begin{array}{l}\text { NM_001090 } \\
611.1\end{array}$ & $919-1018$ & $\begin{array}{l}\text { ACATCCCGTATCCTGGTGGATCAAGTGACAGGTGTTTCTC } \\
\text { GAGGTGTGGGCTTTATCCGGTTTGATAAGAGAATAGAGG } \\
\text { CAGAAGAGGCTATTAAAGGCC }\end{array}$ \\
\hline$f z d 10 . S$ & $\begin{array}{l}\text { NM_001087 } \\
457.1\end{array}$ & $2470-2569$ & $\begin{array}{l}\text { AGCAGTCATGGAAATAGTAGTTAGTTCAGTAGGATCTGGA } \\
\text { TTGCTGCAACAGGGCAGTGCCCACCTAAGTTCTTAGCATG } \\
\text { CGTAGGGTTTGCTTGGCTCT }\end{array}$ \\
\hline gfi1.L & $\begin{array}{l}\text { XM_018258 } \\
576.1\end{array}$ & $735-834$ & $\begin{array}{l}\text { CCTGCTCTGCACGCGGCTGCTAATCAACAACGGCTCATA } \\
\text { CAAATGCCTTAAATGCAGCAAGGTTTTCTCCACACCCCAT } \\
\text { GGGCTTGAAGTCCATGTACGT }\end{array}$ \\
\hline
\end{tabular}




\begin{tabular}{|c|c|c|c|}
\hline hes3.3.S & $\begin{array}{l}\text { XM_018227 } \\
971.1\end{array}$ & $974-1073$ & $\begin{array}{l}\text { ACCCTAGGCTGAACGCAGGCAGGCCGGACTTGCAGCCCT } \\
\text { CTGGCAATTTTGCCCTGAGGAAAACTTGCAAATTCTGCAA } \\
\text { TGGTTTATCCATGGCACTTAA }\end{array}$ \\
\hline hes5.1.S & $\begin{array}{l}\text { NM_001095 } \\
627.1\end{array}$ & $327-426$ & $\begin{array}{l}\text { CAGAAAATGGAGAAACCCAGATGAAACTGCTTAATCACCT } \\
\text { GCAAGCACCCCAAAGGCTCTCAGTTGCCCCTCACACCTA } \\
\text { TATCCCCAGTGTTTCTGACTC }\end{array}$ \\
\hline hes5.2.S & $\begin{array}{l}\text { NM_001095 } \\
626.2\end{array}$ & $339-438$ & $\begin{array}{l}\text { AAGCAGCCAGAAGCAGAAGCCAAGCTAATCAGTCATTTCC } \\
\text { ATTCAAATGCGGCAGCCAGCAGCATGTCGTCTTTTCCTAT } \\
\text { GAGATACAATCAATCCAAGC }\end{array}$ \\
\hline hes9-1.S & $\begin{array}{l}\text { NM_001095 } \\
628.1\end{array}$ & $352-451$ & $\begin{array}{l}\text { GCTTCTGCAAAATTTCCATGATGATGTGACAGCGTGTCCT } \\
\text { TCTGTGTCACCTTTTTACCAGAATCCTGCCAAGTGCACAA } \\
\text { CCCCTGAGGCCAACAAAGTT }\end{array}$ \\
\hline slc18a3.S & $\begin{array}{l}\text { XM_018239 } \\
458.1\end{array}$ & $329-428$ & $\begin{array}{l}\text { CCAAAATGAGGGAGTCCCGCTCCTATCTGTGATGCTACTG } \\
\text { GAGTCACCATGTGAGAGGGGAGGTTCGCTCACCGGCAAT } \\
\text { TGGTTAATGTTCCTCCATGTG }\end{array}$ \\
\hline mxra7.L & $\begin{array}{l}\text { NM_001094 } \\
445.1\end{array}$ & $663-762$ & $\begin{array}{l}\text { ACACATCACCTAGTGGTAGTGGGTGATACTGCAGCGCAA } \\
\text { GATGCTTAAAGACACAGAAACGGTGACCGGCCAATAGAG } \\
\text { TTCTGCAATAACGTGGCAGTGT }\end{array}$ \\
\hline olig2.S & $\begin{array}{l}\text { XM_018248 } \\
885.1\end{array}$ & $1734-1833$ & $\begin{array}{l}\text { TTCGGTTCTGTGTCCCAGACACTGACAATGAACTATCAGC } \\
\text { CTGTAAGTCTTAACTACTTCTACCACAGTCAGGTGCAGAT } \\
\text { CACTTTAAATGCCACCAACA }\end{array}$ \\
\hline pcdh18.L & $\begin{array}{l}\text { XM_018231 } \\
826.1\end{array}$ & $590-689$ & $\begin{array}{l}\text { TTGTGGCAGCTATATTTATCACTTGGTGGCTGTGTACTTG } \\
\text { GACATGACCAGCCTGCTGCCCTCCAGATGTAGTGGATTG } \\
\text { AAGTGTCAGCAGAGTTTCAAT }\end{array}$ \\
\hline pou3f1.S & $\begin{array}{l}\text { NM_001103 } \\
185.1\end{array}$ & $424-523$ & $\begin{array}{l}\text { ATCTACTCGCAGTCCTCCTACACCAACCTGAATGGCATGC } \\
\text { TGGGACCTCAGGCTTCCTCTTTGCACCACAGCATGAGAG } \\
\text { ACCCCTTGCACGATGACCCCG }\end{array}$ \\
\hline$i d 2 . L$ & $\begin{array}{l}\text { NM_001088 } \\
433.1\end{array}$ & $868-967$ & $\begin{array}{l}\text { CGACTTTTTGGGCGATAAAAGGAGCGAAGAAGATCGGGA } \\
\text { AACTTATTTCAGTTTTTGCTCGAAGTGTAAAACCAGAAAAC } \\
\text { CTTGAAACCTTCCCATCCCG }\end{array}$ \\
\hline$i d 4 . S$ & $\begin{array}{l}\text { NM_001171 } \\
975.1\end{array}$ & $149-248$ & $\begin{array}{l}\text { ATGATATGAATGACTGTTACAGCCGGCTCAAGAGGCTCGT } \\
\text { GCCCACCATTCCACCCAACAAGAAAGTCAGCAAAGTGGA } \\
\text { AATCCTGCAGCATGTTATTGA }\end{array}$ \\
\hline insm1.L & $\begin{array}{l}\text { NM_001110 } \\
719.1\end{array}$ & 2178-2277 & $\begin{array}{l}\text { CAAATGACTGTGTCCGCCCATGAGTCGCTTCTACAATGAG } \\
\text { ATAAATGTATATTGCCCAGTTGCCATCCACTCACTGTTACA } \\
\text { GCATTTGTGATGCCCTGAC }\end{array}$ \\
\hline cbfa2t2.S & $\begin{array}{l}\text { NM_001086 } \\
475.1\end{array}$ & $2327-2426$ & $\begin{array}{l}\text { TCATTTGGTTGCTATCAGCTTGCTACAGCCTTCAAATATG } \\
\text { GCCTCAAAGTGCTATCCCAATTATAGACTGCTTACTGGAC } \\
\text { TAAGCCATGTCTGTATTTGG }\end{array}$ \\
\hline chst3.L & $\begin{array}{l}\text { XM_018225 } \\
491.1\end{array}$ & $4782-4881$ & $\begin{array}{l}\text { GGTCATCATTGTATCTATCCAGTAAAAGGTATCACCCAAT } \\
\text { CCACAGGATCCACACTTGTGCCCTTACACATACCTATGCT } \\
\text { AAAAGCAGGTATTTTCCAGA }\end{array}$ \\
\hline prr15l.S & $\begin{array}{l}\text { XM_018238 } \\
024.1\end{array}$ & $249-348$ & $\begin{array}{l}\text { CGTTCCTTTTGTCGTCCATACCCACCAGCTAGAAAAGGTT } \\
\text { GTAGGTCGGGAAGTCTCAGTTGTACATACAGTTTGCCTCA } \\
\text { AGCAAATCTGAAGGGGCTAT }\end{array}$ \\
\hline $\operatorname{six} 3 . L$ & $\begin{array}{l}\text { NM_001085 } \\
702.1\end{array}$ & $777-876$ & $\begin{array}{l}\text { ACAGAGCGGCCGCCGCTAAAAACAGGCTTCAGCACCAAT } \\
\text { CTATCGGACAGAGTGGCATGAGGTCGTTGGCAGATCCCG } \\
\text { GCTGCCCCACACACAGCTCGGC }\end{array}$ \\
\hline snrk.S & $\begin{array}{l}\text { XM_018269 } \\
314.1\end{array}$ & $2348-2447$ & $\begin{array}{l}\text { TCGCCCAGAAGTGCTGGAGAGTTGGTGGAAAGTTTAAAA } \\
\text { CTAATGAGCCTCTGTTTGGGCACGCAGCTACATAACGGG } \\
\text { GCTAAGTATATAATTGACTCTC }\end{array}$ \\
\hline tfap2b.S & $\begin{array}{l}\text { NM_001094 } \\
232.1\end{array}$ & $1243-1342$ & $\begin{array}{l}\text { CTTTGTAAAGAGTTCACAGATCTTTTGGCCCAAGACAGGA } \\
\text { CACCCATAGGCAACAGTAGGCCCAGTCCAATTTTGGAAC } \\
\text { CTGGAATCCAGAGCTGCTTGA }\end{array}$ \\
\hline
\end{tabular}




\begin{tabular}{|c|c|c|c|}
\hline slc43a2.S & $\begin{array}{l}\text { NM_001097 } \\
830.1\end{array}$ & $3239-3338$ & $\begin{array}{l}\text { GGTGACCTACTGTCAGTCTTTTGTGTGATCAGAGGTGAAT } \\
\text { CTGGGAAAATGAATGTTTTGGGAAGCAAAACTCAACTCAG } \\
\text { ATACATCTGGCATCAGGACA }\end{array}$ \\
\hline $\begin{array}{l}\text { Xetrov90021 } \\
170 \mathrm{~m} . \mathrm{L}\end{array}$ & $\begin{array}{l}\text { XM_018232 } \\
445.1\end{array}$ & $1120-1219$ & $\begin{array}{l}\text { TGCCTCTGGCACAGGCTCACCAGACAATTGCTCTTCGTTT } \\
\text { CAAGAGGATGAGGACTTAGATATGGAAAACCTTGGCTGG } \\
\text { CCTGTTAGTAGTTTCACCATC }\end{array}$ \\
\hline $\begin{array}{l}\text { Xelaev1800 } \\
5831 \mathrm{~m} . \mathrm{g}\end{array}$ & $\begin{array}{l}\text { XM_018252 } \\
463.1\end{array}$ & $379-478$ & $\begin{array}{l}\text { TGGATCCCTCAGCACCATGGGTTCAAGGGATTATCAAGAA } \\
\text { AAATTCTCTACAAGCAGGCAACAGAGATAAGTAAGACAAA } \\
\text { GAAGAAGAGCAAGTCTTCGA }\end{array}$ \\
\hline hoxd1.L & $\begin{array}{l}\text { XM_018235 } \\
932.1\end{array}$ & $961-1060$ & $\begin{array}{l}\text { ACGCTTGACTACAGACTCTCAGCTAGAAACCAAATATATG } \\
\text { GGATTGTCTCGTAACACAATGCATACTGCTGCACCCCAAG } \\
\text { CAGCCATAAACTAAGGGGAC }\end{array}$ \\
\hline arid1b.L & $\begin{array}{l}\text { XM_018263 } \\
082.1\end{array}$ & 193-292 & $\begin{array}{l}\text { GCTGAGCACTCTACCAAATGATATGCTTATTGCCAGACTG } \\
\text { TGTCCAAAGTAGGAAAGGGCACCATACATCCTTGGATTCT } \\
\text { TCTTGCTAGAAATCACCTTG }\end{array}$ \\
\hline bhlhe $40 . L$ & $\begin{array}{l}\text { XM_018259 } \\
016.1\end{array}$ & $386-485$ & $\begin{array}{l}\text { CTCACTAGCCCTGGACGAGCCCATTCGCTCCGTCAGGTT } \\
\text { CATGCCACTGACTGGAATACACACACCCACCCGCTCCATT } \\
\text { CCCAAACAGCTGCCACTCGGG }\end{array}$ \\
\hline dmrta2.L & $\begin{array}{l}\text { XM_018258 } \\
408.1\end{array}$ & $629-728$ & $\begin{array}{l}\text { ATAGGGCAGTGAGTGCAGTTATTGGCGCTTTACTGTAGC } \\
\text { GCATAGAGTGTGTATAGAGTTGTACCAGTCAGGGGAGTTT } \\
\text { GTGCCCTTTCCAGTCCCGTGT }\end{array}$ \\
\hline dpys/3.L & $\begin{array}{l}\text { NM_001086 } \\
642.1\end{array}$ & $80-179$ & $\begin{array}{l}\text { AAAAAATACATTGCCTGTATCTGATCAGCTGTAAATCAGTC } \\
\text { AGGGGAAGCAGAAGCAGCGGGCTCGGAGGCAAAACATG } \\
\text { TCTTACCAGGGGAAGAAGAAC }\end{array}$ \\
\hline efnb1.L & $\begin{array}{l}\text { NM_001087 } \\
479.2\end{array}$ & $110-209$ & $\begin{array}{l}\text { TTATATCTGGAAAGGGCCTGGTTCTATACCCTGAAATTGG } \\
\text { CGACCGCTTGGACATTATCTGCCCCAAGGGGGACTCTTC } \\
\text { CCAGCCTTATGAATACTATAA }\end{array}$ \\
\hline gfra1.S & $\begin{array}{l}\text { XM_018227 } \\
240.1\end{array}$ & $1934-2033$ & $\begin{array}{l}\text { ACAAGATCGAGAACAACCAATTCGACTCTAACAGAGGAAA } \\
\text { AATAAAATAAAGACTGCTCTCTGGTATTTAGGGGGTGTTG } \\
\text { TAATTTATCACTGAGGTGCC }\end{array}$ \\
\hline nes.L & $\begin{array}{l}\text { XM_018231 } \\
440.1\end{array}$ & 6793-6892 & $\begin{array}{l}\text { GTACTGTAAGGCTACAGGGTCTTTACAGGCTTGGGATATT } \\
\text { GAGGGTTTAAAATGTTAGCTGTATGGAAATGGGTTGATTC } \\
\text { TTAGGAATGGCAGCTGAGTG }\end{array}$ \\
\hline neurod4.L & $\begin{array}{l}\text { NM_001087 } \\
744.1\end{array}$ & $666-765$ & $\begin{array}{l}\text { AGTAATGGATCCTTCTGTGGTAACCCATACACTTAACTGT } \\
\text { ACCACTCCACCATATGAAGGAGCTCTAACACCTCCACTCA } \\
\text { GCATCGGTGGTAATTTTTCT }\end{array}$ \\
\hline plk3.L & $\begin{array}{l}\text { XM_018258 } \\
343.1\end{array}$ & 2967-3066 & $\begin{array}{l}\text { GACTGTTTTTGACGGAACACAAAGCAGACCAGCAATATGC } \\
\text { AACACTTTGTGTAAGAGTAGAGAAAACACTACAGTTGTGG } \\
\text { ATCACTTCAATCAGCAGACC }\end{array}$ \\
\hline snai2.L & $\begin{array}{l}\text { NM_001086 } \\
282.1\end{array}$ & $554-653$ & $\begin{array}{l}\text { CTCTACTTTCTCTGGGTTGGCTAAGCACAAGCAGCTGCAT } \\
\text { TGCGACGCCCAGTCTCGGAAATCATTTAGCTGCAAGTACT } \\
\text { GTGAAAAGGAGTATGTGAGC }\end{array}$ \\
\hline sox9.L & $\begin{array}{l}\text { NM_001094 } \\
473.1\end{array}$ & $163-262$ & $\begin{array}{l}\text { CTGACTTGGCCACTTCCATTGTTCTCGGTTAAGTTGCGCA } \\
\text { AAGACGCGGCTCTGCGCAAACAGTGCCACCCGCAGCCG } \\
\text { ACGAACTCGCTTTTCCAACATT }\end{array}$ \\
\hline tox3.L & $\begin{array}{l}\text { XM_018257 } \\
839.1\end{array}$ & $557-656$ & $\begin{array}{l}\text { ACAAACCGAACAGCTAGCTCCTACCACAGAGACTCCTCTC } \\
\text { TAGATTTTTGTGCGATTGTAAATGCCGAGTAACTGGAAGA } \\
\text { TCTGCCACCAGCCTTTACGC }\end{array}$ \\
\hline vcan.L & $\begin{array}{l}\text { NM_001110 } \\
715.1\end{array}$ & $433-532$ & $\begin{array}{l}\text { TCTTTGGATGTTTCAGGAGTCGTGTTTCACTACCGAGCCT } \\
\text { CTACAGACAAGTACTCTTTGGACTTTGAGGCTGCTCAGAA } \\
\text { AGCTTGTATAGACAGCGGTG }\end{array}$ \\
\hline pou3f2.L & $\begin{array}{l}\text { NM_001096 } \\
751.1\end{array}$ & $29-128$ & $\begin{array}{l}\text { GCTCAGCATCCCGCAGGTTGGAAAAGGGTGGGCGGGAC } \\
\text { TGTTTGGATCATCATCCAATGGCTACGGCGGAAAGTCTCC } \\
\text { GGTTGCTAGAGGTATCCACGTA }\end{array}$ \\
\hline
\end{tabular}




\begin{tabular}{|c|c|c|c|}
\hline scrt1.S & $\begin{array}{l}\text { XM_018238 } \\
286.1\end{array}$ & $4316-4415$ & $\begin{array}{l}\text { TAGGCAGGGACTACATATTAGCCGTGTGCTCTTTCTCCAC } \\
\text { CCTATAGTATTTCTTTGGAACAGTTGGAAGGGGCTACTTA } \\
\text { TGAGACTCACGCTCTTCCAT }\end{array}$ \\
\hline hesx1.S & $\begin{array}{l}\text { NM_001085 } \\
795.1\end{array}$ & $169-268$ & $\begin{array}{l}\text { TTGAGTGCAGCAGCAAGGTTGACGGCACCTTTTGGCAAA } \\
\text { TCCCATTGATCAGCTATGACCTTCCCGTACAAGTTGACGC } \\
\text { CATGCGCCGATCCGCAGAAGA }\end{array}$ \\
\hline $\operatorname{drgx} . L$ & $\begin{array}{l}\text { XM_018225 } \\
618.1\end{array}$ & $12-111$ & $\begin{array}{l}\text { ACATGGGACTAGGCAGACAGCGCTAATTCTGACCCGAAG } \\
\text { GGAAGACAAGACTTCCCCTCGAGGATCAAGTCAGCGATC } \\
\text { ATGAGCATGACCATAGCTCCCC }\end{array}$ \\
\hline b4galnt1.S & $\begin{array}{l}\text { XM_018250 } \\
090.1\end{array}$ & 2683-2782 & $\begin{array}{l}\text { TCTGAAGCGCAACTTAGTAAGAGCAAGTAAGAGCCTAGG } \\
\text { ATGCTAGAAAAGAAAGACCTCAACTCCTCCAACCTACATA } \\
\text { ATCACCAAAGCTCCTACCCAT }\end{array}$ \\
\hline map3k12.S & $\begin{array}{l}\text { NM_001100 } \\
941.1\end{array}$ & $1870-1969$ & $\begin{array}{l}\text { AGAGGAATGTACCTCAGAAATTATCCCCACATGGGAAAAG } \\
\text { GCCAGACATTTTGAAATCTGAAGTCCTGCTGCCAAAGGTA } \\
\text { GACTCTGTCTTGTCACCCTC }\end{array}$ \\
\hline tubb3.L & $\begin{array}{l}\text { NM_001094 } \\
986.1\end{array}$ & $1642-1741$ & $\begin{array}{l}\text { AGGTACTGCACCTTAATAACCCTTTTAACGCTTGCCAGTTT } \\
\text { CAGCAGCAGAGCAATGTTCAGCATTCAACAACATATCATT } \\
\text { GCAGGCTCCAGTGAACAGA }\end{array}$ \\
\hline hoxc4.S & $\begin{array}{l}\text { XM_018250 } \\
129.1\end{array}$ & 483-582 & $\begin{array}{l}\text { TTGGAATGAGCCTCTGGTTCCTTATCCGGGGACCTGAGC } \\
\text { GCACGTTGTGATTGGCTGCCGGAGTCACATGGTGAAAGT } \\
\text { AACTTTACGGGGTCGCCAGCTA }\end{array}$ \\
\hline kif26a.L & $\begin{array}{l}\text { XM_018230 } \\
605.1\end{array}$ & $118-217$ & $\begin{array}{l}\text { TTATAAGTATTGATTGATACCCACCTGCCTCTTATTGCACG } \\
\text { GGCTGCACTTCCATTACCACGTGATTGACTACACGACTAA } \\
\text { GTGCTTTCCTTGTAGCTGT }\end{array}$ \\
\hline eya1.L & $\begin{array}{l}\text { NM_001090 } \\
419.1\end{array}$ & $820-919$ & $\begin{array}{l}\text { ACAGCCGAATACAGCACTATCCACAGTCCATCAACGCCTA } \\
\text { TTAAAGATTCAGATTCAGATCGATTGCGGCGCAGTTCGGA } \\
\text { TGGAAAGTCACGGGGACGAG }\end{array}$ \\
\hline bgn.L & $\begin{array}{l}\text { NM_001090 } \\
709.1\end{array}$ & $1208-1307$ & $\begin{array}{l}\text { GTTGGCGTCAATGACTTCTGCCCTATAGGATTCGGAGTGA } \\
\text { AACGCGCCTACTACAACGGAATTAGTCTATTTAACAACCC } \\
\text { CGTGCCCTACTGGGAAGTGC }\end{array}$ \\
\hline $\begin{array}{l}\text { Xelaev1804 } \\
\text { 0877m.g }\end{array}$ & $\begin{array}{l}\text { NM_001086 } \\
467.1\end{array}$ & $1086-1185$ & $\begin{array}{l}\text { CAACCCCAACGGGATCATTGCCACACCCATCACCCAAATT } \\
\text { AACCCAATCACTTCTTCTTCAGGTACCAGTACTCCACCAA } \\
\text { CACTCACTGCCACTCAAGTA }\end{array}$ \\
\hline $\begin{array}{l}\text { LOC100487 } \\
395 . L\end{array}$ & $\begin{array}{l}\text { XM_004911 } \\
907.2\end{array}$ & $5272-5371$ & $\begin{array}{l}\text { GCCTTACCACTGACTTCCTCTACTTTGGCCATTTTCTGATA } \\
\text { GGTTACACCCATCTCCGGCCAAGTCCAGAGTCTCTGTAGT } \\
\text { TGTGCTGTGACCTAACAGG }\end{array}$ \\
\hline gas6.L & $\begin{array}{l}\text { NM_001089 } \\
688.1\end{array}$ & $1811-1910$ & $\begin{array}{l}\text { TCCTTTGATGGGACCCTCGGACTAAAAGAACTGCCTGATT } \\
\text { CACAGATGCAGATGACTTTGCTTCTGCTAAATGACCACTT } \\
\text { AGGAAAGGGTGTAAAGACAT }\end{array}$ \\
\hline Ibx1.S & $\begin{array}{l}\text { XM_018227 } \\
455.1\end{array}$ & $272-371$ & $\begin{array}{l}\text { TTCTTTTACCTTCTCCCTGGCCAAAGGATGAATTTAGCTG } \\
\text { GGAGTAGGCTGAATTCTGTTTGAAGGGAAGGAGCCTGAG } \\
\text { ATTGCACAAGAAACATCCAGC }\end{array}$ \\
\hline $\begin{array}{l}\text { Xelaev1800 } \\
7508 \mathrm{~m} . \mathrm{g}\end{array}$ & $\begin{array}{l}\text { XR_001935 } \\
594.1\end{array}$ & $572-671$ & $\begin{array}{l}\text { CTTCAACAGGATGTGCAGAAGTGAGCACCAAGCACTGGT } \\
\text { AAGATTGCATACACAGAGCTGCAAGAGAGTCCTGGTACTT } \\
\text { ATCCATACAAGACCTCCAGAG }\end{array}$ \\
\hline map2.S & $\begin{array}{l}\text { NM_001086 } \\
770.1\end{array}$ & $1188-1287$ & $\begin{array}{l}\text { CCACAAACCAGGTGGTGGACATGTGAGGATTGAAAGTGT } \\
\text { AAAACTTGATTTCAAGGAGAAAGCACAGGCTAAAATTGGC } \\
\text { TCTTTAGACAATGCCAGTCAT }\end{array}$ \\
\hline$m m p 28 . L$ & $\begin{array}{l}\text { NM_001172 } \\
229.2\end{array}$ & $14-113$ & $\begin{array}{l}\text { GGTGAGCAGCTACAGGTCTCAATCCAAGGACTATACCAT } \\
\text { GCCTGGCTGAGAAGAACAGCAGAAGTCTCTGATAACTCT } \\
\text { GCCAAACTCTACACTTGGGAAG }\end{array}$ \\
\hline ncam1.S & $\begin{array}{l}\text { NM_001087 } \\
827.1\end{array}$ & $45-144$ & $\begin{array}{l}\text { CATCTGGACTTTATATTTCATAGGAACTGCAGTGGCGTTG } \\
\text { GAAGTGAACATTGTTCCAGATCAAGGAGAAATAAGCCTTG } \\
\text { GGGAGTCCAAATTCTTCCTG }\end{array}$ \\
\hline
\end{tabular}




\begin{tabular}{|c|c|c|c|}
\hline otx2.L & $\begin{array}{l}\text { NM_001090 } \\
691.1\end{array}$ & $43-142$ & $\begin{array}{l}\text { TCCACTTGTAGAAAATTGTGCGCAAAAAAAAATAAGAAAAA } \\
\text { ACGCCCAACGGATCCAGCAAACACTGATCGCCCGACTTT } \\
\text { GTCGCTGCAACGATTTCTTC }\end{array}$ \\
\hline pck1.L & $\begin{array}{l}\text { NM_001086 } \\
683.1\end{array}$ & $2306-2405$ & $\begin{array}{l}\text { TGTAGGGTGGATGTACAAAGCAAAGGCGGTATAGTTTGT } \\
\text { GCCTCTTCTTTATTAGAGGTGGGTCAGTCTAGTGAAATAA } \\
\text { GACTCAATCCAGATTGTCACT }\end{array}$ \\
\hline ppp1r9b.S & $\begin{array}{l}\text { XM_018238 } \\
072.1\end{array}$ & $3927-4026$ & $\begin{array}{l}\text { TCCGGCTGCCCCAGATATCCTAGCACCTCTCATGACCCTC } \\
\text { CCGTTGTGTTAAAATTCCACTGTAAGAATGCAGAGGAGGT } \\
\text { TCGCTGTTTGGGGGTGACAT }\end{array}$ \\
\hline snap25.L & $\begin{array}{l}\text { NM_001090 } \\
411.1\end{array}$ & $495-594$ & $\begin{array}{l}\text { TAGAGAAACAGAAATGGATGAGAACCTTGAGCAAGTCGG } \\
\text { TGGCATTATTGGAAATCTTCGTCACATGGCCCTCGATATG } \\
\text { GGCAATGAGATTGACACACAA }\end{array}$ \\
\hline sox11.L & $\begin{array}{l}\text { NM_001142 } \\
362.1\end{array}$ & $593-692$ & $\begin{array}{l}\text { AGCGGCAGCGGCTCCAAGTCGCTCAGTATCAAGTCCGAG } \\
\text { TACAGCGGCGGCAGCGACGAGTATGTGTTCGGCAGCCC } \\
\text { CAAAGCGTCCGGCAAAGCTGCGG }\end{array}$ \\
\hline prph.S & $\begin{array}{l}\text { NM_001086 } \\
386.1\end{array}$ & $227-326$ & $\begin{array}{l}\text { AAGGTCCAGTACCCCTGTCCGAGTCTCATTGGACCGAGT } \\
\text { GGACTTCTCTGCGGCTGAGGCTGTTAACCAAGAATTCCTG } \\
\text { ACCACCCGCAGTAATGAGAAA }\end{array}$ \\
\hline slc3a2.L & $\begin{array}{l}\text { NM_001085 } \\
977.1\end{array}$ & $855-954$ & $\begin{array}{l}\text { AGAGTCCTGTTGTTATCCACAAGTAGTGCTCAAAACAACC } \\
\text { TTACTGGTGGCTTCAATGAGACAATAGATGGCACGCTCTT } \\
\text { CTACCGCTTCCTGGGTGCTG }\end{array}$ \\
\hline runx1t1.L & $\begin{array}{l}\text { NM_001095 } \\
596.1\end{array}$ & $650-749$ & $\begin{array}{l}\text { ACCTTCATCCTCTTCGTCATCGTCGCTGGCCAATCAGCAG } \\
\text { TTGCCTCCGGCTTGTGGCGCACGGCAGCTCAGTAAGCTC } \\
\text { AAGCGATTCCTCACTACATTG }\end{array}$ \\
\hline$d m b x 1 . S$ & $\begin{array}{l}\text { XM_018260 } \\
870.1\end{array}$ & 2128-2227 & $\begin{array}{l}\text { TGCGAAAAGGAAATGCTTTAAGCTGTAGGGAAGAAAACACT } \\
\text { TTTGGTGTGGACTGTGTTCCGCAGAGACTAGATGCCATAC } \\
\text { TTTTCCTCTGACTGAATGCC }\end{array}$ \\
\hline cacna2d2.L & $\begin{array}{l}\text { XM_018258 } \\
986.1\end{array}$ & $5458-5557$ & $\begin{array}{l}\text { ACCTCCCTGTCATACAGTACGGTACCAAAGAAGCAGGTTT } \\
\text { TGACTAAACGTGTTGCATCTCTTCGGCGTAACGTGTCACA } \\
\text { TCTGAACAGGAAGCAATGGC }\end{array}$ \\
\hline rapgef5.L & $\begin{array}{l}\text { XM_018267 } \\
363.1\end{array}$ & 1998-2097 & $\begin{array}{l}\text { CTGCGGGGAAAAACATGAGCTTCTACCAAATGAATTGGTC } \\
\text { CTCTCCAAAACCCTGGAGCCCTCCAACCGTATTTATACTT } \\
\text { ACAGGAAGAGTGACACATTG }\end{array}$ \\
\hline k/h/14.L & $\begin{array}{l}\text { XM_018267 } \\
966.1\end{array}$ & $524-623$ & $\begin{array}{l}\text { CATTATTGCTACTCGCACATTCCTGCTCTCCCTCGGCGGC } \\
\text { TCATTCACTACTAGGAGCACCATGTTGATGATATACGGAC } \\
\text { AAATACACTGTGAGGAGTGA }\end{array}$ \\
\hline fam102b.L & $\begin{array}{l}\text { XM_018258 } \\
650.1\end{array}$ & $3715-3814$ & $\begin{array}{l}\text { GTGCTGCTGATTCACTATAAGAGCAAGTATATTGGCAGTA } \\
\text { GTTCAAAGGGCAGAGGCCAAAACAATTAAAATCGGTGCC } \\
\text { ATTCAGTTGTATGGCAGATAC }\end{array}$ \\
\hline adamts20.L & $\begin{array}{l}\text { XM_018251 } \\
404.1\end{array}$ & $3770-3869$ & $\begin{array}{l}\text { CAGCTTCTTGTGGGAGGGGTAATCGTGCTCGATATGTGA } \\
\text { GTTGTAGGGATGCGTTTGGTGGAGTTGCTGAAGAACTGTT } \\
\text { TTGCGCCCATTTTCCACGACC }\end{array}$ \\
\hline
\end{tabular}




\subsection{GO tables RNA sequencing experiments}

\subsubsection{RNA sequencing experiment onset of target gene activation by}

\section{Ptf1a}

Gene Ontology (GO) analysis was performed using DAVID (http://david.abcc. ncifcrf.gov) as previously described (Dennis et al., 2003). Analyzed were genes which were upregulated after 3,6 and $12 \mathrm{~h}$, respectively.

\subsubsection{GO analysis of candidate genes upregulated after 3,6 or $12 \mathrm{~h}$.} Given is the biological process, the number of genes and the P-value. Biological processes were sorted according to the P-value from lowest to highest.

Table S8: Summary of GO analysis of genes upregulated after $\mathbf{3 h}$. Shown is the top 20 enriched categories in Biological Processes

\begin{tabular}{|l|c|l|}
\hline Term & Count & PValue \\
\hline GO:0007275 multicellular organism development & 29 & $1.67 \mathrm{E}-10$ \\
\hline GO:0045944 positive regulation of transcription from RNA polymerase II promoter & 25 & $4.33 \mathrm{E}-08$ \\
\hline GO:0000122 negative regulation of transcription from RNA polymerase II promoter & 21 & $8.74 \mathrm{E}-08$ \\
\hline GO:0045893 positive regulation of transcription, DNA-templated & 17 & $1.65 \mathrm{E}-06$ \\
\hline GO:0006351 transcription, DNA-templated & 32 & $1.93 \mathrm{E}-06$ \\
\hline GO:0045665 negative regulation of neuron differentiation & 7 & $1.24 \mathrm{E}-05$ \\
\hline GO:0006355 regulation of transcription, DNA-templated & 34 & $1.30 \mathrm{E}-05$ \\
\hline GO:0007399 nervous system development & 12 & $4.97 \mathrm{E}-05$ \\
\hline GO:0048663 neuron fate commitment & 5 & $6.13 \mathrm{E}-05$ \\
\hline GO:0042472 inner ear morphogenesis & 6 & $1.26 \mathrm{E}-04$ \\
\hline GO:0045892 negative regulation of transcription, DNA-templated & 14 & $1.47 \mathrm{E}-04$ \\
\hline GO:0007386 compartment pattern specification & 3 & $2.69 \mathrm{E}-04$ \\
\hline GO:0001657 ureteric bud development & 5 & $3.05 \mathrm{E}-04$ \\
\hline GO:0090090 negative regulation of canonical Wnt signaling pathway & 6 & $6.36 \mathrm{E}-04$ \\
\hline GO:0008045 motor neuron axon guidance & 4 & $8.68 \mathrm{E}-04$ \\
\hline GO:0014807 regulation of somitogenesis & 3 & $1.23 \mathrm{E}-03$ \\
\hline GO:0060612 adipose tissue development & 4 & $1.54 \mathrm{E}-03$ \\
\hline GO:0001822 kidney development & 6 & $1.88 \mathrm{E}-03$ \\
\hline GO:0045747 positive regulation of Notch signaling pathway & 4 & $1.97 \mathrm{E}-03$ \\
\hline GO:0043507 positive regulation of JUN kinase activity & $2.47 \mathrm{E}-03$ \\
\hline
\end{tabular}


Table S9: Summary of GO analysis of genes upregulated after $6 \mathrm{~h}$. Shown is the top 20 enriched categories in Biological Processes

\begin{tabular}{|l|c|l|}
\hline Term & Count & PValue \\
\hline GO:0007275 multicellular organism development & 57 & $1.15 \mathrm{E}-20$ \\
\hline GO:0006351 transcription, DNA-templated & 62 & $8.09 \mathrm{E}-12$ \\
\hline GO:0045944 positive regulation of transcription from RNA polymerase II promoter & 42 & $3.68 \mathrm{E}-11$ \\
\hline GO:0006355 regulation of transcription, DNA-templated & 67 & $1.17 \mathrm{E}-10$ \\
\hline GO:0007399 nervous system development & 25 & $1.40 \mathrm{E}-10$ \\
\hline GO:0000122 negative regulation of transcription from RNA polymerase II promoter & 34 & $3.61 \mathrm{E}-10$ \\
\hline GO:0045893 positive regulation of transcription, DNA-templated & 29 & $1.84 \mathrm{E}-09$ \\
\hline GO:0045665 negative regulation of neuron differentiation & 11 & $4.92 \mathrm{E}-08$ \\
\hline GO:0001822 kidney development & 13 & $1.25 \mathrm{E}-07$ \\
\hline GO:0048665 neuron fate specification & 6 & $9.40 \mathrm{E}-07$ \\
\hline GO:0045892 negative regulation of transcription, DNA-templated & 24 & $1.95 \mathrm{E}-06$ \\
\hline GO:0007219 Notch signaling pathway & 11 & $5.26 \mathrm{E}-06$ \\
\hline GO:0030182 neuron differentiation & 10 & $4.12 \mathrm{E}-05$ \\
\hline GO:0010628 positive regulation of gene expression & 17 & $6.68 \mathrm{E}-05$ \\
\hline GO:0071599 otic vesicle development & 4 & $7.21 \mathrm{E}-05$ \\
\hline GO:0030154 cell differentiation & 25 & $7.94 \mathrm{E}-05$ \\
\hline GO:0061314 Notch signaling involved in heart development & 4 & $1.14 \mathrm{E}-04$ \\
\hline GO:0014807 regulation of somitogenesis & 4 & $1.14 \mathrm{E}-04$ \\
\hline GO:0010977 negative regulation of neuron projection development & 7 & $1.76 \mathrm{E}-04$ \\
\hline GO:0010629 negative regulation of gene expression & 13 & $1.82 \mathrm{E}-04$ \\
\hline
\end{tabular}

Table S10: Summary of GO analysis of genes upregulated after $12 \mathrm{~h}$. Shown is the top 20 enriched categories in Biological Processes

\begin{tabular}{|l|c|l|}
\hline Term & Count & PValue \\
\hline GO:0007275 multicellular organism development & 93 & $9.40 \mathrm{E}-25$ \\
\hline GO:0007399 nervous system development & 52 & $1.45 \mathrm{E}-21$ \\
\hline GO:0006351 transcription, DNA-templated & 107 & $1.89 \mathrm{E}-13$ \\
\hline GO:0006355 regulation of transcription, DNA-templated & 119 & $1.64 \mathrm{E}-12$ \\
\hline GO:0030154 cell differentiation & 54 & $8.26 \mathrm{E}-10$ \\
\hline GO:0030182 neuron differentiation & 20 & $1.59 \mathrm{E}-09$ \\
\hline $\begin{array}{l}\text { GO:0000122 negative regulation of transcription from RNA } \\
\text { polymerase Il promoter }\end{array}$ & 51 & $1.88 \mathrm{E}-09$ \\
\hline GO:0014032 neural crest cell development & 9 & $5.56 \mathrm{E}-09$ \\
\hline $\begin{array}{l}\text { GO:0045893 positive regulation of transcription, DNA- } \\
\text { templated }\end{array}$ & 43 & $6.98 \mathrm{E}-09$ \\
\hline $\begin{array}{l}\text { GO:0045944 positive regulation of transcription from RNA } \\
\text { polymerase II promoter }\end{array}$ & 59 & $3.46 \mathrm{E}-08$ \\
\hline GO:0001822 kidney development & 18 & $8.94 \mathrm{E}-08$ \\
\hline GO:0007411 axon guidance & 18 & $6.53 \mathrm{E}-07$ \\
\hline GO:0009952 anterior/posterior pattern specification & 15 & $2.13 \mathrm{E}-06$ \\
\hline
\end{tabular}




\begin{tabular}{|l|l|l|}
\hline GO:0045165 cell fate commitment & 12 & $3.66 \mathrm{E}-06$ \\
\hline GO:0001501 skeletal system development & 14 & $6.74 \mathrm{E}-06$ \\
\hline $\begin{array}{l}\text { GO:0045892 negative regulation of transcription, DNA- } \\
\text { templated }\end{array}$ & 36 & $9.39 \mathrm{E}-06$ \\
\hline GO:0001756 somitogenesis & 10 & $1.85 \mathrm{E}-05$ \\
\hline GO:0007507 heart development & 21 & $3.31 \mathrm{E}-05$ \\
\hline $\begin{array}{l}\text { GO:0010977 negative regulation of neuron projection } \\
\text { development }\end{array}$ & 10 & $5.54 \mathrm{E}-05$ \\
\hline GO:0001649 osteoblast differentiation & 13 & $5.90 \mathrm{E}-05$ \\
\hline
\end{tabular}

\subsubsection{GO analysis of direct target genes having their onset at 3, 6 or} $12 \mathrm{~h}$.

Given is the biological process, the number of genes and the P-value. Biological processes were sorted according to the P-value from lowest to highest

\section{Table S11: Summary of GO analysis of direct target genes having their onset at}

$\mathbf{3} \mathbf{h}$. Shown is the top 20 enriched categories in Biological Processes

\begin{tabular}{|c|c|c|}
\hline Term & Count & PValue \\
\hline $\begin{array}{l}\text { GO:0045944 positive regulation of transcription from RNA polymerase II } \\
\text { promoter }\end{array}$ & 10 & $2.99 \mathrm{E}-05$ \\
\hline GO:0006351 transcription, DNA-templated & 13 & 3.37E-05 \\
\hline $\begin{array}{l}\text { GO:0000122 negative regulation of transcription from RNA polymerase II } \\
\text { promoter }\end{array}$ & 8 & $1.90 \mathrm{E}-04$ \\
\hline GO:0006355 regulation of transcription, DNA-templated & 13 & 2.17E-04 \\
\hline GO:0045665 negative regulation of neuron differentiation & 4 & $2.95 \mathrm{E}-04$ \\
\hline GO:0010628 positive regulation of gene expression & 6 & $5.40 \mathrm{E}-04$ \\
\hline GO:0007275 multicellular organism development & 8 & $\begin{array}{l}0.00149290 \\
3\end{array}$ \\
\hline GO:0043507 positive regulation of JUN kinase activity & 3 & $\begin{array}{l}0.00213050 \\
4\end{array}$ \\
\hline GO:0061074 regulation of neural retina development & 2 & 0.00513470 \\
\hline GO:0007420 brain development & 4 & $\begin{array}{l}0.00590557 \\
2\end{array}$ \\
\hline GO:0051302 regulation of cell division & 2 & $\begin{array}{l}0.01024389 \\
6\end{array}$ \\
\hline GO:0001701 in utero embryonic development & 4 & $\begin{array}{l}0.01429242 \\
1\end{array}$ \\
\hline GO:0032308 positive regulation of prostaglandin secretion & 2 & $\begin{array}{l}0.01532768 \\
8\end{array}$ \\
\hline GO:0045893 positive regulation of transcription, DNA-templated & 5 & $\begin{array}{l}0.01621132 \\
8\end{array}$ \\
\hline GO:0045892 negative regulation of transcription, DNA-templated & 5 & $\begin{array}{l}0.01649368 \\
9\end{array}$ \\
\hline GO:0007399 nervous system development & 4 & $\begin{array}{l}0.02625220 \\
5\end{array}$ \\
\hline GO:0042491 auditory receptor cell differentiation & 2 & 0.02709179 \\
\hline GO:0060042 retina morphogenesis in camera-type eye & 2 & 0.02709179 \\
\hline GO:0014032 neural crest cell development & 2 & $\begin{array}{l}0.02876123 \\
3\end{array}$ \\
\hline GO:0050880 regulation of blood vessel size & 2 & $\begin{array}{l}0.03209180 \\
6\end{array}$ \\
\hline
\end{tabular}


Table S12: Summary of GO analysis of direct target genes having their onset at

\section{$6 \mathbf{h}$. Shown is the top 20 enriched categories in Biological Processes}

\begin{tabular}{|l|c|l|}
\hline Term & Count & PValue \\
\hline GO:0006351 transcription, DNA-templated & 21 & $4.03 \mathrm{E}-07$ \\
\hline $\begin{array}{l}\text { GO:0045944 positive regulation of transcription from RNA polymerase II } \\
\text { promoter }\end{array}$ & 15 & $1.41 \mathrm{E}-06$ \\
\hline $\begin{array}{l}\text { GO:0000122 negative regulation of transcription from RNA polymerase II } \\
\text { promoter }\end{array}$ & 13 & $1.84 \mathrm{E}-06$ \\
\hline GO:0006355 regulation of transcription, DNA-templated & 21 & $7.97 \mathrm{E}-06$ \\
\hline GO:0010628 positive regulation of gene expression & 9 & $2.95 \mathrm{E}-05$ \\
\hline GO:0051302 regulation of cell division & 3 & $1.40 \mathrm{E}-04$ \\
\hline GO:0007399 nervous system development & 8 & $1.56 \mathrm{E}-04$ \\
\hline GO:0007219 Notch signaling pathway & 5 & $6.05 \mathrm{E}-04$ \\
\hline GO:0048666 neuron development & 4 & $7.69 \mathrm{E}-04$ \\
\hline GO:0010977 negative regulation of neuron projection development & 4 & $\begin{array}{l}0.00102543 \\
9\end{array}$ \\
\hline GO:0007275 multicellular organism development & 11 & $\begin{array}{l}0.00110944 \\
5\end{array}$ \\
\hline GO:0007405 neuroblast proliferation & 3 & $\begin{array}{l}0.00155735 \\
3\end{array}$ \\
\hline GO:0001701 in utero embryonic development & 6 & $\begin{array}{l}0.00229267 \\
5\end{array}$ \\
\hline GO:0045840 positive regulation of mitotic nuclear division & 3 & $\begin{array}{l}0.00362493 \\
3\end{array}$ \\
\hline GO:0043507 positive regulation of JUN kinase activity & 3 & 0.00681414 \\
\hline GO:0031018 endocrine pancreas development & 3 & $\begin{array}{l}0.00714943 \\
3\end{array}$ \\
\hline GO:0045893 positive regulation of transcription, DNA-templated & 7 & $\begin{array}{l}0.00857335 \\
3\end{array}$ \\
\hline GO:0045892 negative regulation of transcription, DNA-templated & 2 & $\begin{array}{l}0.00878360 \\
2\end{array}$ \\
\hline GO:0060741 prostate gland stromal morphogenesis & $\begin{array}{l}0.00926277 \\
4\end{array}$ \\
\hline GO:0001660 fever generation & 2 & 0.01539108 \\
7
\end{tabular}

Table S13: Summary of GO analysis of direct target genes having their onset at $12 \mathrm{~h}$.

\begin{tabular}{|l|c|l|}
\hline Term & Count & PValue \\
\hline GO:0098903 regulation of membrane repolarization during action potential & 2 & 0.005135 \\
\hline $\begin{array}{l}\text { GO:0061577 calcium ion transmembrane transport via high voltage-gated } \\
\text { calcium channel }\end{array}$ & 2 & 0.005135 \\
\hline GO:0007275 multicellular organism development & 7 & 0.007235 \\
\hline GO:0030182 neuron differentiation & 3 & 0.019926 \\
\hline GO:0060402 calcium ion transport into cytosol & 2 & 0.020386 \\
\hline GO:0007399 nervous system development & 4 & 0.026252 \\
\hline GO:0007411 axon guidance & 3 & 0.026829 \\
\hline GO:0006351 transcription, DNA-templated & 8 & 0.037116 \\
\hline GO:0009755 hormone-mediated signaling pathway & 2 & 0.042017 \\
\hline GO:0042127 regulation of cell proliferation & 3 & 0.057504 \\
\hline GO:0016358 dendrite development & 2 & 0.059959 \\
\hline GO:0007017 microtubule-based process & 2 & 0.068006 \\
\hline
\end{tabular}




\subsubsection{RNA sequencing experiment Brg1 knock-down}

Gene Ontology (GO) analysis was performed using DAVID (http://david.abcc. ncifcrf.gov) as previously described (Dennis et al., 2003). Analyzed were genes which were affected by a Brg1 knock-down at stage 14

Table S14: Summary of GO analysis Brg1 affected candidate genes. Shown is the top 20 enriched categories in Biological Processes

\begin{tabular}{|c|c|c|}
\hline Term & Count & PValue \\
\hline GO:0007275 multicellular organism development & 11 & $5.15 E-04$ \\
\hline $\begin{array}{l}\text { GO:0045944 positive regulation of transcription from RNA polymerase II } \\
\text { promoter }\end{array}$ & 10 & 0.001680363 \\
\hline GO:0003333 amino acid transmembrane transport & 3 & 0.002068881 \\
\hline GO:0007420 brain development & 5 & 0.003190223 \\
\hline GO:0010975 regulation of neuron projection development & 3 & 0.003225707 \\
\hline GO:0042127 regulation of cell proliferation & 5 & 0.003809389 \\
\hline GO:0007017 microtubule-based process & 3 & 0.00596161 \\
\hline GO:0006865 amino acid transport & 3 & 0.006248464 \\
\hline GO:0009953 dorsal/ventral pattern formation & 3 & 0.008427991 \\
\hline GO:0006351 transcription, DNA-templated & 12 & 0.014441686 \\
\hline GO:0015807 L-amino acid transport & 2 & 0.016806335 \\
\hline GO:0050680 negative regulation of epithelial cell proliferation & 3 & 0.017579689 \\
\hline GO:0021766 hippocampus development & 3 & 0.018042667 \\
\hline GO:0045665 negative regulation of neuron differentiation & 3 & 0.019462391 \\
\hline GO:0021798 forebrain dorsal/ventral pattern formation & 2 & 0.019580338 \\
\hline GO:0007399 nervous system development & 5 & 0.02153236 \\
\hline GO:0030154 cell differentiation & 7 & 0.021850607 \\
\hline GO:0021978 telencephalon regionalization & 2 & 0.022346667 \\
\hline GO:0001843 neural tube closure & 3 & 0.03062912 \\
\hline GO:0030336 negative regulation of cell migration & 3 & 0.036028973 \\
\hline
\end{tabular}




\subsection{RNA-seq data}

\subsubsection{Candidate gene list for the RNA sequencing analysis of Ptf1a-GR} time course

Given are the genes which are differentially expressed between Ptf1a-GR overexpressing animal caps over non-injected control caps after 3, 6 and $12 \mathrm{~h}$ in two individual replicates. Given are the gene ID, the $\log _{2} \mathrm{FC}$ activation over $\mathrm{CC}$ and the p-value.

Table S15: Summary of differentially expressed genes activates after $3 \mathbf{h}$

\begin{tabular}{|c|c|c|}
\hline ID & log2FC & P-value \\
\hline ptf1a.L & 7.97 & $1.39 \mathrm{E}-44$ \\
\hline$z c 3 h 12 c . L$ & 6.21 & 8.02E-38 \\
\hline hes5.1.L & 6.19 & $1.55 \mathrm{E}-28$ \\
\hline prdm13.S & 5.62 & $8.35 \mathrm{E}-17$ \\
\hline zc3h12c.S & 5.40 & 3.36E-23 \\
\hline hes5.1.S & 5.13 & $9.48 \mathrm{E}-43$ \\
\hline hes5_X2.S & 5.12 & $7.14 \mathrm{E}-11$ \\
\hline$e b f 2 . L$ & 4.93 & $3.11 \mathrm{E}-16$ \\
\hline prr15l.S & 4.77 & $4.79 \mathrm{E}-07$ \\
\hline grb14.L & 4.75 & $3.79 \mathrm{E}-11$ \\
\hline slc18a3.S & 4.73 & $1.75 \mathrm{E}-12$ \\
\hline$n k x 2-1 . L$ & 4.71 & $3.29 \mathrm{E}-05$ \\
\hline cbfa2t2.S & 4.57 & $1.62 \mathrm{E}-31$ \\
\hline hes5.2.S & 4.55 & 1.67E-11 \\
\hline dlc.L & 4.50 & $2.01 \mathrm{E}-13$ \\
\hline il1b.S & 4.48 & $4.19 \mathrm{E}-07$ \\
\hline myt1.L & 4.47 & $2.50 \mathrm{E}-24$ \\
\hline hes5.3.S & 4.35 & $1.02 \mathrm{E}-14$ \\
\hline mxra7.L & 4.24 & $3.29 \mathrm{E}-10$ \\
\hline hes9-1.L & 4.13 & 8.82E-08 \\
\hline fzd10.S & 4.12 & $1.34 \mathrm{E}-19$ \\
\hline rbm24.L & 4.08 & $4.59 \mathrm{E}-28$ \\
\hline LOC100494099.S & 4.00 & 5.02E-04 \\
\hline thbs1.L & 3.99 & $2.46 \mathrm{E}-24$ \\
\hline pcdh18.L & 3.97 & $5.08 \mathrm{E}-14$ \\
\hline foxc1.L & 3.87 & $8.53 \mathrm{E}-08$ \\
\hline$n h s / 2 . L$ & 3.81 & $5.66 \mathrm{E}-08$ \\
\hline
\end{tabular}




\begin{tabular}{|c|c|c|}
\hline hes5.2.L & 3.76 & $6.74 \mathrm{E}-11$ \\
\hline LOC100495335.L & 3.72 & $2.78 \mathrm{E}-05$ \\
\hline hepacam2.L & 3.69 & $2.30 \mathrm{E}-10$ \\
\hline dII1.L & 3.67 & $7.75 \mathrm{E}-21$ \\
\hline Xelaev18037602m.g & 3.64 & $1.65 \mathrm{E}-10$ \\
\hline hoxd1.L & 3.61 & $5.49 \mathrm{E}-06$ \\
\hline gpr63.S & 3.52 & $1.78 \mathrm{E}-14$ \\
\hline hes9-2.L & 3.52 & $2.55 \mathrm{E}-05$ \\
\hline Irrn1-like.1.S & 3.51 & $2.48 \mathrm{E}-13$ \\
\hline arl4a.S & 3.50 & 1.23E-29 \\
\hline prdm13.L & 3.49 & $3.34 \mathrm{E}-05$ \\
\hline LOC101733706-like.L & 3.48 & $6.55 \mathrm{E}-06$ \\
\hline post.L & 3.45 & $7.62 \mathrm{E}-12$ \\
\hline hes10.L & 3.44 & $3.01 \mathrm{E}-07$ \\
\hline Xetrov90021170m.L & 3.41 & $7.76 \mathrm{E}-25$ \\
\hline$s p 5 . L$ & 3.38 & $3.16 \mathrm{E}-07$ \\
\hline adam33.S & 3.37 & $5.14 \mathrm{E}-05$ \\
\hline aldh1b1.L & 3.35 & $1.43 \mathrm{E}-03$ \\
\hline gfi1.L & 3.28 & 5.02E-04 \\
\hline post.S & 3.25 & $3.21 \mathrm{E}-10$ \\
\hline Irrn1-like.1.L & 3.23 & $1.31 \mathrm{E}-25$ \\
\hline id4.S & 3.21 & 7.30E-15 \\
\hline pzp.L & 3.21 & $6.20 \mathrm{E}-05$ \\
\hline dll1.S & 3.20 & $1.76 \mathrm{E}-10$ \\
\hline hes3.3.S & 3.19 & $3.61 \mathrm{E}-06$ \\
\hline cd247.S & 3.17 & $8.11 \mathrm{E}-04$ \\
\hline Irrc36.L & 3.16 & 5.17E-08 \\
\hline fstl1.S & 3.15 & $7.74 \mathrm{E}-04$ \\
\hline hes5_X1.L & 3.14 & $5.54 \mathrm{E}-08$ \\
\hline tfap2b.S & 3.14 & 1.86E-03 \\
\hline pou3f1.L & 3.08 & $7.70 \mathrm{E}-06$ \\
\hline dlk2.L & 3.07 & 4.47E-05 \\
\hline hes6.2.L & 3.03 & $2.12 \mathrm{E}-02$ \\
\hline hes9-1.S & 3.03 & $1.02 \mathrm{E}-09$ \\
\hline LOC101733157.L & 3.03 & $2.52 \mathrm{E}-04$ \\
\hline ebf2.S & 3.02 & $9.24 \mathrm{E}-06$ \\
\hline insm1.S & 3.02 & $7.99 \mathrm{E}-06$ \\
\hline elavl3.S & 3.01 & $3.63 \mathrm{E}-08$ \\
\hline
\end{tabular}




\begin{tabular}{|c|c|c|}
\hline kcnn1.S & 3.01 & 8.87E-04 \\
\hline mmp11.S & 3.00 & $3.40 \mathrm{E}-02$ \\
\hline olig2.S & 2.99 & $2.43 \mathrm{E}-02$ \\
\hline c8orf46.S & 2.98 & $1.53 \mathrm{E}-02$ \\
\hline sybu.L & 2.98 & $1.01 \mathrm{E}-09$ \\
\hline Xelaev18035340m.g & 2.98 & $4.48 \mathrm{E}-07$ \\
\hline myt1.S & 2.92 & $2.25 \mathrm{E}-06$ \\
\hline zbtb18.S & 2.92 & $1.01 \mathrm{E}-09$ \\
\hline$c d c 25 b . L$ & 2.89 & $1.17 \mathrm{E}-10$ \\
\hline $\operatorname{Imcd1.L}$ & 2.86 & $2.34 \mathrm{E}-02$ \\
\hline id4.L & 2.82 & $2.55 \mathrm{E}-03$ \\
\hline LOC100487224.L & 2.81 & $1.34 \mathrm{E}-08$ \\
\hline numbl.L & 2.81 & $1.00 \mathrm{E}-10$ \\
\hline LOC100127750.S & 2.80 & $1.12 \mathrm{E}-04$ \\
\hline cbfa2t2.L & 2.72 & $1.71 \mathrm{E}-11$ \\
\hline fgf16.L & 2.72 & $6.54 \mathrm{E}-03$ \\
\hline npnt.S & 2.70 & $2.39 \mathrm{E}-04$ \\
\hline LOC100489305.S & 2.67 & $9.28 \mathrm{E}-03$ \\
\hline spo11.L & 2.67 & $7.50 \mathrm{E}-03$ \\
\hline tril.S & 2.65 & $2.90 \mathrm{E}-07$ \\
\hline Xelaev18032619m.g & 2.63 & $9.71 \mathrm{E}-04$ \\
\hline $\operatorname{six} 3 . L$ & 2.62 & $7.74 \mathrm{E}-04$ \\
\hline hes8.L & 2.61 & $2.74 \mathrm{E}-02$ \\
\hline tiparp.L & 2.61 & $1.72 \mathrm{E}-20$ \\
\hline tsc22d3.L & 2.56 & $4.11 \mathrm{E}-06$ \\
\hline prr7.L & 2.53 & $8.16 \mathrm{E}-04$ \\
\hline myb.S & 2.52 & 1.17E-06 \\
\hline Ihx4.S & 2.51 & $4.05 \mathrm{E}-02$ \\
\hline pou3f1.S & 2.51 & $7.32 \mathrm{E}-08$ \\
\hline ankrd65.L & 2.50 & $4.79 \mathrm{E}-03$ \\
\hline Xelaev18007001m.g & 2.49 & $4.41 \mathrm{E}-07$ \\
\hline slc43a2.S & 2.47 & $3.01 \mathrm{E}-07$ \\
\hline Ca2.L & 2.44 & $1.57 \mathrm{E}-02$ \\
\hline Xelaev18000619m.g & 2.44 & $2.21 \mathrm{E}-02$ \\
\hline amotl2.L & 2.41 & $3.17 \mathrm{E}-16$ \\
\hline hes5_X2.L & 2.39 & $3.00 \mathrm{E}-05$ \\
\hline $\operatorname{lh} \times 1 . S$ & 2.36 & $4.47 \mathrm{E}-05$ \\
\hline atp6v1c2.L & 2.35 & $4.65 \mathrm{E}-04$ \\
\hline
\end{tabular}




\begin{tabular}{|c|c|c|}
\hline$m m p 17 . L$ & 2.35 & $4.90 \mathrm{E}-02$ \\
\hline nek2.L & 2.35 & $1.32 \mathrm{E}-03$ \\
\hline egr4.L & 2.33 & $6.54 \mathrm{E}-03$ \\
\hline manea.L & 2.33 & $1.25 \mathrm{E}-02$ \\
\hline sec24d-like.1.L & 2.32 & 4.19E-04 \\
\hline kitlg.S & 2.25 & $3.81 \mathrm{E}-03$ \\
\hline Xelaev18005831m.g & 2.23 & $1.16 \mathrm{E}-03$ \\
\hline edn1.L & 2.22 & $1.46 \mathrm{E}-02$ \\
\hline fam198b.L & 2.21 & $2.73 \mathrm{E}-06$ \\
\hline ampd2.S & 2.17 & 4.05E-02 \\
\hline$r b / 2 . L$ & 2.17 & $1.64 \mathrm{E}-02$ \\
\hline$d k k 2 . L$ & 2.16 & 4.05E-02 \\
\hline znf534.L & 2.14 & $2.44 \mathrm{E}-03$ \\
\hline ap/nr.L & 2.13 & $4.52 \mathrm{E}-03$ \\
\hline axin2.L & 2.13 & $3.93 \mathrm{E}-02$ \\
\hline foxi1.L & 2.12 & $1.86 \mathrm{E}-03$ \\
\hline gjc2.L & 2.12 & 6.77E-03 \\
\hline insm1.L & 2.12 & $1.96 \mathrm{E}-03$ \\
\hline lancl3.S & 2.12 & $4.51 \mathrm{E}-02$ \\
\hline tp73.S & 2.10 & $2.03 \mathrm{E}-02$ \\
\hline rab15.L & 2.09 & $5.19 \mathrm{E}-04$ \\
\hline LOC101731452.L & 2.07 & $5.12 \mathrm{E}-05$ \\
\hline stard4.L & 2.07 & 7.74E-04 \\
\hline fam101b.L & 2.06 & $2.44 \mathrm{E}-03$ \\
\hline$m x i 1 . L$ & 2.05 & $1.16 \mathrm{E}-04$ \\
\hline$m y b . L$ & 2.05 & 5.17E-04 \\
\hline vash2.S & 2.05 & $1.28 \mathrm{E}-05$ \\
\hline fzd10.L & 2.03 & 3.57E-03 \\
\hline prickle1.S & 2.03 & $4.02 \mathrm{E}-08$ \\
\hline cdknx.L & 2.02 & $1.35 \mathrm{E}-07$ \\
\hline
\end{tabular}

Table S16: Summary of differentially expressed genes activates after 6 h

\begin{tabular}{|l|c|c|}
\hline ID & log2FC & P-value \\
\hline ptf1a.L & 8.52 & $3,81 \mathrm{E}-43$ \\
\hline prdm13.S & 7.86 & $3,91 \mathrm{E}-34$ \\
\hline prdm13.L & 7.36 & $7,60 \mathrm{E}-23$ \\
\hline
\end{tabular}




\begin{tabular}{|c|c|c|}
\hline$e b f 2 . L$ & 7.08 & $9,35 E-33$ \\
\hline gfi1.L & 6.31 & 9,57E-13 \\
\hline pou3f1.L & 6.27 & $2,34 \mathrm{E}-20$ \\
\hline mxra7.L & 6.03 & $6,25 \mathrm{E}-17$ \\
\hline pou3f1.S & 5.88 & $1,21 \mathrm{E}-33$ \\
\hline cbfa2t2.S & 5.78 & $2,21 \mathrm{E}-50$ \\
\hline LOC100127750.S & 5.69 & $3,79 \mathrm{E}-11$ \\
\hline zc3h12c.L & 5.62 & $2,98 \mathrm{E}-38$ \\
\hline hes5.2.L & 5.6 & $7,18 \mathrm{E}-13$ \\
\hline prr15l.S & 5.57 & $2,06 \mathrm{E}-09$ \\
\hline hes8.L & 5.55 & $4,89 \mathrm{E}-14$ \\
\hline ebf2.S & 5.48 & $2,28 \mathrm{E}-17$ \\
\hline hes5.1.L & 5.46 & $2,69 \mathrm{E}-27$ \\
\hline neurod4.L & 5.36 & $2,16 \mathrm{E}-09$ \\
\hline grb14.L & 5.26 & $1,29 \mathrm{E}-08$ \\
\hline LOC100494099.S & 5.22 & $1,99 \mathrm{E}-07$ \\
\hline fzd10.S & 5.11 & $4,26 \mathrm{E}-33$ \\
\hline hes8.S & 5.11 & $3,17 \mathrm{E}-12$ \\
\hline zc3h12c.S & 5.09 & $5,61 \mathrm{E}-23$ \\
\hline id4.S & 4.99 & $2,69 \mathrm{E}-46$ \\
\hline gpr63.S & 4.96 & $5,62 \mathrm{E}-18$ \\
\hline dmrt2.L & 4.93 & $6,25 \mathrm{E}-17$ \\
\hline hepacam2.L & 4.91 & $1,55 \mathrm{E}-17$ \\
\hline elavl3.S & 4.88 & $1,32 \mathrm{E}-17$ \\
\hline hes3.3.S & 4.84 & $6,69 \mathrm{E}-19$ \\
\hline Xelaev18007001m.g & 4.84 & $9,10 \mathrm{E}-25$ \\
\hline cd247.S & 4.82 & $4,15 \mathrm{E}-08$ \\
\hline slc18a3.S & 4.78 & $3,58 \mathrm{E}-09$ \\
\hline thbs1.L & 4.69 & $2,19 \mathrm{E}-26$ \\
\hline chst3.L & 4.6 & $2,63 \mathrm{E}-15$ \\
\hline olig2.S & 4.58 & $4,78 \mathrm{E}-06$ \\
\hline hes5.1.S & 4.56 & 2,92E-35 \\
\hline$p z p . L$ & 4.54 & $4,14 \mathrm{E}-09$ \\
\hline b4galnt1.S & 4.53 & $6,59 \mathrm{E}-10$ \\
\hline hes9-1.L & 4.47 & $9,16 \mathrm{E}-09$ \\
\hline fam101b.L & 4.45 & $8,32 \mathrm{E}-14$ \\
\hline Ihx1.S & 4.45 & $5,62 \mathrm{E}-18$ \\
\hline hoxd1.L & 4.44 & $2,51 \mathrm{E}-08$ \\
\hline
\end{tabular}




\begin{tabular}{|c|c|c|}
\hline hes5_X2.S & 4.42 & $5,18 \mathrm{E}-10$ \\
\hline runx1t1.L & 4.39 & 1,85E-09 \\
\hline igf2.L & 4.36 & $3,14 \mathrm{E}-07$ \\
\hline ptger3.L & 4.35 & 1,26E-06 \\
\hline hes5_X1.L & 4.34 & $1,81 \mathrm{E}-13$ \\
\hline hes5.3.S & 4.29 & $5,26 \mathrm{E}-13$ \\
\hline c8orf46.S & 4.27 & $2,17 \mathrm{E}-06$ \\
\hline foxc1.L & 4.26 & $1,55 \mathrm{E}-07$ \\
\hline tcf15.L & 4.19 & 1,33E-06 \\
\hline myt1.S & 4.17 & $7,72 \mathrm{E}-14$ \\
\hline c8orf46.L & 4.16 & $6,26 \mathrm{E}-04$ \\
\hline zbtb18.S & 4.13 & $2,29 \mathrm{E}-17$ \\
\hline myt1.L & 4.1 & $4,67 \mathrm{E}-21$ \\
\hline$t / x 3 . S$ & 4.03 & $1,58 \mathrm{E}-07$ \\
\hline nhsl2.L & 4.02 & $2,52 \mathrm{E}-07$ \\
\hline six1.L & 4.02 & $9,30 \mathrm{E}-05$ \\
\hline $\operatorname{lh} \times 4 . S$ & 3.98 & $2,77 \mathrm{E}-06$ \\
\hline hes10.L & 3.97 & $1,59 \mathrm{E}-09$ \\
\hline mamdc2.L & 3.96 & $5,76 \mathrm{E}-06$ \\
\hline dlk2.L & 3.93 & $4,14 \mathrm{E}-09$ \\
\hline numbl.L & 3.93 & $1,06 \mathrm{E}-22$ \\
\hline $\operatorname{drgx} . L$ & 3.85 & $1,75 \mathrm{E}-04$ \\
\hline scrt1.S & 3.84 & $7,49 \mathrm{E}-07$ \\
\hline Xelaev18036651m.g & 3.84 & $9,19 \mathrm{E}-04$ \\
\hline cbfa2t2.L & 3.78 & $3,44 \mathrm{E}-23$ \\
\hline Xelaev18036200m.g & 3.78 & $9,23 \mathrm{E}-06$ \\
\hline hes6.2.L & 3.77 & $2,81 \mathrm{E}-04$ \\
\hline fstl1.S & 3.72 & $1,16 \mathrm{E}-04$ \\
\hline bmp3.L & 3.71 & $2,15 \mathrm{E}-05$ \\
\hline Xelaev18037602m.g & 3.71 & $7,13 \mathrm{E}-11$ \\
\hline$c d c 25 b . L$ & 3.7 & $4,76 \mathrm{E}-18$ \\
\hline il1b.S & 3.67 & $2,87 \mathrm{E}-04$ \\
\hline sybu.L & 3.65 & $5,63 \mathrm{E}-13$ \\
\hline$m m p 17 . L$ & 3.63 & $1,12 \mathrm{E}-04$ \\
\hline kitlg.S & 3.59 & $2,90 \mathrm{E}-07$ \\
\hline egr4.L & 3.58 & $7,11 \mathrm{E}-06$ \\
\hline zc3h12a.L & 3.55 & $6,53 \mathrm{E}-03$ \\
\hline plk3.L & 3.51 & $3,72 \mathrm{E}-13$ \\
\hline
\end{tabular}




\begin{tabular}{|c|c|c|}
\hline $\operatorname{drgx.S}$ & 3.48 & 4,14E-05 \\
\hline Xelaev18028850m.g & 3.48 & $7,23 \mathrm{E}-11$ \\
\hline hnf1b.S & 3.44 & 3,31E-03 \\
\hline hes6.2.S & 3.43 & $6,91 \mathrm{E}-03$ \\
\hline myos-like.S & 3.41 & 6,38E-03 \\
\hline Xetrov90021170m.L & 3.38 & 9,93E-25 \\
\hline hes9-1.S & 3.37 & $2,09 \mathrm{E}-11$ \\
\hline adam33.S & 3.36 & 5,10E-05 \\
\hline gfra1.S & 3.36 & 9,67E-04 \\
\hline twist1.L & 3.36 & 9,70E-04 \\
\hline edn1.L & 3.35 & $2,10 \mathrm{E}-05$ \\
\hline gfra3.S & 3.35 & $1,42 \mathrm{E}-02$ \\
\hline relt.S & 3.35 & 2,04E-05 \\
\hline Xelaev18005831m.g & 3.31 & $1,58 \mathrm{E}-07$ \\
\hline arl4a.S & 3.3 & $2,08 \mathrm{E}-26$ \\
\hline LOC100036671.L & 3.29 & $3,67 \mathrm{E}-04$ \\
\hline dpys/3.L & 3.28 & $3,88 \mathrm{E}-04$ \\
\hline Irrn1-like.1.S & 3.28 & $7,50 \mathrm{E}-17$ \\
\hline pou3f3.L & 3.27 & $4,64 \mathrm{E}-03$ \\
\hline s/c43a2.S & 3.27 & $2,10 \mathrm{E}-11$ \\
\hline tril.S & 3.26 & $2,71 \mathrm{E}-10$ \\
\hline dmrta2.S & 3.24 & $8,19 \mathrm{E}-06$ \\
\hline$f z d 10 . L$ & 3.23 & $1,56 \mathrm{E}-08$ \\
\hline LOC101733706-like.L & 3.23 & $2,44 \mathrm{E}-03$ \\
\hline rltpr.L & 3.23 & $1,06 \mathrm{E}-04$ \\
\hline ecel1.S & 3.22 & $2,47 \mathrm{E}-02$ \\
\hline insm1.S & 3.22 & $6,55 \mathrm{E}-07$ \\
\hline$m m p 11 . L$ & 3.22 & $5,56 \mathrm{E}-03$ \\
\hline Xelaev18036111m.g & 3.21 & $1,19 \mathrm{E}-07$ \\
\hline shd.L & 3.2 & $5,62 \mathrm{E}-03$ \\
\hline aldh1b1.L & 3.18 & $4,59 \mathrm{E}-03$ \\
\hline LOC100495335.L & 3.16 & $3,93 \mathrm{E}-04$ \\
\hline zc3h12a.S & 3.16 & $7,72 \mathrm{E}-03$ \\
\hline tIr3.L & 3.15 & $2,21 \mathrm{E}-02$ \\
\hline LOC101733157.L & 3.14 & $1,38 \mathrm{E}-03$ \\
\hline foxa1.S & 3.13 & $2,47 \mathrm{E}-10$ \\
\hline tfe3.L & 3.13 & $1,15 \mathrm{E}-02$ \\
\hline$r b m 24 . L$ & 3.12 & $6,25 \mathrm{E}-17$ \\
\hline
\end{tabular}




\begin{tabular}{|c|c|c|}
\hline hes9-2.L & 3.09 & 7,34E-04 \\
\hline LOC100485259.L & 3.09 & $2,97 \mathrm{E}-17$ \\
\hline arhgef26.L & 3.06 & $4,73 \mathrm{E}-06$ \\
\hline neurod4.S & 3.06 & $1,06 \mathrm{E}-03$ \\
\hline Xelaev18019047m.g & 3.06 & $2,20 \mathrm{E}-03$ \\
\hline$h m \times 3 . L$ & 3.05 & $1,44 \mathrm{E}-02$ \\
\hline$s p 5 . L$ & 3.05 & $2,72 \mathrm{E}-06$ \\
\hline rassf6.L & 3.04 & $2,33 \mathrm{E}-02$ \\
\hline s/c3a2.L & 3.02 & $1,15 \mathrm{E}-02$ \\
\hline Xelaev18032400m.g & 3.02 & $4,09 \mathrm{E}-02$ \\
\hline snrk.S & 3.01 & $3,94 \mathrm{E}-15$ \\
\hline$n p t \times 2 . L$ & 3 & $3,50 \mathrm{E}-02$ \\
\hline axin2.S & 2.99 & $6,69 \mathrm{E}-03$ \\
\hline ag1.S & 2.97 & 3,67E-09 \\
\hline fgf16.L & 2.97 & $1,52 \mathrm{E}-03$ \\
\hline $\operatorname{tn} 3 . L$ & 2.97 & $1,58 \mathrm{E}-02$ \\
\hline muc1.L & 2.96 & $2,99 \mathrm{E}-02$ \\
\hline prr7.L & 2.95 & $2,35 \mathrm{E}-05$ \\
\hline$e m \times 1 . L$ & 2.94 & $4,10 \mathrm{E}-02$ \\
\hline gfi1.S & 2.94 & $1,02 \mathrm{E}-04$ \\
\hline rab15.L & 2.93 & $3,03 \mathrm{E}-10$ \\
\hline p2ry4.L & 2.91 & $1,71 \mathrm{E}-02$ \\
\hline eln2.L & 2.9 & $2,46 \mathrm{E}-04$ \\
\hline axin2.L & 2.88 & $6,17 \mathrm{E}-04$ \\
\hline faxc.L & 2.88 & $1,21 \mathrm{E}-24$ \\
\hline Xetrov90002103m.L & 2.87 & $3,20 \mathrm{E}-02$ \\
\hline cfap70.L & 2.85 & $7,65 \mathrm{E}-03$ \\
\hline Xelaev18000619m.g & 2.83 & $1,57 \mathrm{E}-03$ \\
\hline prr18.L & 2.82 & $1,95 \mathrm{E}-03$ \\
\hline bh/he22.L & 2.81 & $1,15 \mathrm{E}-02$ \\
\hline angpt/4.L & 2.79 & $9,30 \mathrm{E}-05$ \\
\hline galnt12.L & 2.78 & $3,56 \mathrm{E}-07$ \\
\hline ccdc141.L & 2.77 & $1,62 \mathrm{E}-03$ \\
\hline pou2f3.L & 2.77 & $2,81 E-09$ \\
\hline snai2.L & 2.76 & $8,04 \mathrm{E}-05$ \\
\hline $\operatorname{irx3.L}$ & 2.75 & $3,20 \mathrm{E}-04$ \\
\hline LOC100493614.S & 2.74 & $8,14 \mathrm{E}-07$ \\
\hline amot/2.S & 2.72 & $4,42 \mathrm{E}-16$ \\
\hline
\end{tabular}




\begin{tabular}{|c|c|c|}
\hline phlda1.L & 2.72 & $1,50 \mathrm{E}-03$ \\
\hline gch1.L & 2.7 & $3,51 \mathrm{E}-13$ \\
\hline tfap2b.S & 2.69 & $2,89 \mathrm{E}-02$ \\
\hline post.L & 2.68 & $1,56 \mathrm{E}-07$ \\
\hline LOC100494099.L & 2.67 & $1,41 \mathrm{E}-02$ \\
\hline mdk.S & 2.66 & 1,27E-05 \\
\hline ncmap.S & 2.64 & $8,17 \mathrm{E}-03$ \\
\hline post.S & 2.64 & $3,20 \mathrm{E}-07$ \\
\hline bhlhe40.L & 2.63 & $2,43 E-03$ \\
\hline Xelaev18005924m.g & 2.63 & $2,41 \mathrm{E}-06$ \\
\hline insm1.L & 2.62 & 1,31E-05 \\
\hline frem2.S & 2.6 & $1,02 \mathrm{E}-04$ \\
\hline hes3.1.S & 2.6 & $2,50 \mathrm{E}-09$ \\
\hline rab15.S & 2.57 & $3,20 \mathrm{E}-02$ \\
\hline arhgap23.L & 2.56 & $4,08 \mathrm{E}-05$ \\
\hline eya1.S & 2.56 & $2,54 \mathrm{E}-06$ \\
\hline rnf165.L & 2.56 & $4,03 \mathrm{E}-08$ \\
\hline vtcn1.L & 2.56 & 1,15E-03 \\
\hline hes5.2.S & 2.55 & $2,87 \mathrm{E}-02$ \\
\hline lypd6.L & 2.55 & $4,38 \mathrm{E}-03$ \\
\hline fjx1.S & 2.54 & $1,68 \mathrm{E}-02$ \\
\hline kremen2.S & 2.53 & $2,85 \mathrm{E}-02$ \\
\hline spo11.L & 2.53 & $1,53 \mathrm{E}-02$ \\
\hline Xetrov90025030m.L & 2.53 & 4,37E-05 \\
\hline Imcd1.L & 2.5 & $4,34 \mathrm{E}-02$ \\
\hline gria1-like.1.S & 2.49 & $4,75 \mathrm{E}-02$ \\
\hline kctd12.L & 2.49 & $1,18 \mathrm{E}-02$ \\
\hline Irrc36.L & 2.49 & $1,08 \mathrm{E}-05$ \\
\hline meis1.L & 2.48 & $4,50 \mathrm{E}-05$ \\
\hline hes5_X2.L & 2.47 & $3,78 \mathrm{E}-05$ \\
\hline Xetrov90000859m.L & 2.47 & $2,05 \mathrm{E}-02$ \\
\hline LOC100497338-like.L & 2.45 & 1,27E-05 \\
\hline vcan.L & 2.44 & $7,74 \mathrm{E}-03$ \\
\hline ctsk.L & 2.43 & $1,68 \mathrm{E}-02$ \\
\hline Xelaev18042455m.g & 2.43 & 5,79E-03 \\
\hline pm20d1.L & 2.42 & $1,88 \mathrm{E}-05$ \\
\hline tfap2e.S & 2.42 & 6,37E-03 \\
\hline elavl4.L & 2.41 & $1,15 \mathrm{E}-02$ \\
\hline
\end{tabular}




\begin{tabular}{|c|c|c|}
\hline Ihx8.L & 2.41 & $4,91 \mathrm{E}-06$ \\
\hline prickle1.S & 2.4 & $2,09 \mathrm{E}-11$ \\
\hline bhlhe40.S & 2.39 & $2,71 \mathrm{E}-03$ \\
\hline tiparp.L & 2.38 & $4,71 \mathrm{E}-16$ \\
\hline slc1a1.L & 2.37 & $3,03 E-02$ \\
\hline$d b p . S$ & 2.36 & 8,24E-05 \\
\hline irf1.L & 2.35 & $1,83 \mathrm{E}-07$ \\
\hline cidec.L & 2.33 & $1,19 \mathrm{E}-16$ \\
\hline glipr2.S & 2.33 & 3,98E-05 \\
\hline Xelaev18026372m.g & 2.32 & $7,78 \mathrm{E}-08$ \\
\hline cdknx.L & 2.31 & $2,71 \mathrm{E}-10$ \\
\hline onecut1.2.L & 2.31 & 3,39E-03 \\
\hline a2m.S & 2.3 & $4,67 \mathrm{E}-16$ \\
\hline amotl2.L & 2.3 & $1,85 \mathrm{E}-15$ \\
\hline $\operatorname{aim1I.S}$ & 2.28 & $1,14 \mathrm{E}-02$ \\
\hline syt16.L & 2.28 & $1,23 \mathrm{E}-03$ \\
\hline fam214a.S & 2.27 & $2,20 \mathrm{E}-05$ \\
\hline kcnc4.S & 2.27 & $1,00 \mathrm{E}-02$ \\
\hline Xelaev18013182m.g & 2.27 & $6,39 \mathrm{E}-04$ \\
\hline id4.L & 2.25 & 5,22E-05 \\
\hline plk3.S & 2.25 & $6,95 \mathrm{E}-05$ \\
\hline atf $6 b . S$ & 2.24 & 7,67E-04 \\
\hline rnf125.L & 2.23 & $2,85 E-02$ \\
\hline znf238.2.L & 2.21 & $6,04 \mathrm{E}-20$ \\
\hline sept5.L & 2.2 & $3,04 \mathrm{E}-04$ \\
\hline SOX9.L & 2.19 & $7,42 \mathrm{E}-03$ \\
\hline dll1.L & 2.18 & $2,12 \mathrm{E}-07$ \\
\hline sec24d-like.1.L & 2.17 & $5,16 \mathrm{E}-03$ \\
\hline serpinf1.L & 2.17 & $4,44 \mathrm{E}-02$ \\
\hline znf534.L & 2.17 & 3,73E-04 \\
\hline pcdh18.L & 2.15 & $4,14 \mathrm{E}-04$ \\
\hline iqca1.S & 2.14 & $2,11 \mathrm{E}-02$ \\
\hline reep5.S & 2.14 & $4,38 \mathrm{E}-05$ \\
\hline znf238.2.S & 2.13 & $6,45 \mathrm{E}-09$ \\
\hline kdm7a.L & 2.11 & $6,68 \mathrm{E}-04$ \\
\hline C8orf4.L & 2.1 & $1,21 \mathrm{E}-02$ \\
\hline creb3/2.S & 2.1 & $3,25 \mathrm{E}-03$ \\
\hline rfk.S & 2.1 & $4,10 \mathrm{E}-02$ \\
\hline
\end{tabular}




\begin{tabular}{|l|c|c|}
\hline atp6v0d2.L & 2.09 & $4,81 \mathrm{E}-02$ \\
\hline dnase1/3.L & 2.09 & $2,12 \mathrm{E}-02$ \\
\hline eps8/2.L & 2.08 & $3,64 \mathrm{E}-02$ \\
\hline nes.L & 2.08 & $2,37 \mathrm{E}-04$ \\
\hline fam83e.S & 2.06 & $6,05 \mathrm{E}-03$ \\
\hline Xelaev18023501m.g & 2.06 & $3,34 \mathrm{E}-07$ \\
\hline ankrd65.L & 2.05 & $3,80 \mathrm{E}-02$ \\
\hline gse1.L & 2.02 & $3,04 \mathrm{E}-05$ \\
\hline rap1gap.S & 2.01 & $1,47 \mathrm{E}-02$ \\
\hline xk.L & 2.01 & $2,82 \mathrm{E}-02$ \\
\hline
\end{tabular}

Table S17: Summary of differentially expressed genes activates after $12 \mathrm{~h}$

\begin{tabular}{|l|c|c|}
\hline ID & log2FC & p-value \\
\hline prdm13.L & 9.38 & $1,04 \mathrm{E}-37$ \\
\hline prdm13.S & 9.33 & $3,02 \mathrm{E}-45$ \\
\hline neurod4.L & 8.84 & $4,86 \mathrm{E}-27$ \\
\hline scrt1.S & 8.55 & $2,29 \mathrm{E}-31$ \\
\hline aldh1b1.L & 8.4 & $3,23 \mathrm{E}-28$ \\
\hline ptf1a.L & 8.38 & $5,09 \mathrm{E}-43$ \\
\hline chst3.L & 8.06 & $4,49 \mathrm{E}-28$ \\
\hline ebf2.L & 7.91 & $8,93 \mathrm{E}-34$ \\
\hline ebf2.S & 7.85 & $8,56 \mathrm{E}-27$ \\
\hline neurod4.S & 7.56 & $2,54 \mathrm{E}-20$ \\
\hline dpys/3.L & 7.44 & $3,18 \mathrm{E}-47$ \\
\hline pck1.L & 7.39 & $5,55 \mathrm{E}-22$ \\
\hline Xetrov90027705m.L & 7.24 & $4,51 \mathrm{E}-15$ \\
\hline ppp1r9b.S & 7.1 & $3,61 \mathrm{E}-22$ \\
\hline tfap2b.S & 7.06 & $1,27 \mathrm{E}-24$ \\
\hline bgn.L & 7.05 & $2,26 \mathrm{E}-31$ \\
\hline mxra7.L & 6.89 & $1,57 \mathrm{E}-24$ \\
\hline shd.L & 6.71 & $1,88 \mathrm{E}-19$ \\
\hline slc18a3.S & 6.53 & $4,21 \mathrm{E}-25$ \\
\hline Ibx1.S & 6.52 & $5,90 \mathrm{E}-11$ \\
\hline drgx.L & 6.45 & $7,42 \mathrm{E}-13$ \\
\hline pou3f1.L & $8,84 \mathrm{E}-18$ \\
\hline hesx1.S & $4,28 \mathrm{E}-27$ \\
\hline
\end{tabular}




\begin{tabular}{|c|c|c|}
\hline$m m p 28 . L$ & 6.28 & $6,27 \mathrm{E}-13$ \\
\hline Xelaev18040877m.g & 6.28 & $5,95 \mathrm{E}-16$ \\
\hline$n p b . L$ & 6.23 & $1,25 \mathrm{E}-09$ \\
\hline LOC100490531-like.L & 6.22 & $7,15 \mathrm{E}-10$ \\
\hline igfbp/1.L & 6.17 & $3,59 \mathrm{E}-11$ \\
\hline prph.S & 5.94 & $5,31 \mathrm{E}-37$ \\
\hline LOC100487395.L & 5.91 & $9,09 \mathrm{E}-22$ \\
\hline tubb3.L & 5.91 & $1,47 \mathrm{E}-11$ \\
\hline igf2.L & 5.82 & $9,07 \mathrm{E}-13$ \\
\hline kirrel2.L & 5.78 & $6,60 \mathrm{E}-41$ \\
\hline pou3f1.S & 5.77 & $2,20 \mathrm{E}-18$ \\
\hline Xelaev18032183m.g & 5.7 & $5,63 \mathrm{E}-08$ \\
\hline dpys/4.L & 5.63 & $3,67 \mathrm{E}-08$ \\
\hline nr5a2.L & 5.59 & $1,25 \mathrm{E}-08$ \\
\hline cd247.S & 5.55 & $3,82 \mathrm{E}-11$ \\
\hline snap25.L & 5.52 & $5,63 \mathrm{E}-12$ \\
\hline kirrel2.S & 5.5 & $2,41 \mathrm{E}-37$ \\
\hline tfap2b.L & 5.49 & $1,52 \mathrm{E}-07$ \\
\hline map2.S & 5.43 & $1,82 \mathrm{E}-22$ \\
\hline onecut1.2.L & 5.4 & $4,03 E-40$ \\
\hline nova1.L & 5.17 & $7,10 \mathrm{E}-13$ \\
\hline pyy.L & 5.17 & $4,41 \mathrm{E}-06$ \\
\hline elavl4.L & 5.16 & $5,31 \mathrm{E}-15$ \\
\hline LOC101733706-like.L & 5.16 & 1,61E-09 \\
\hline myl1.L & 5.14 & $1,00 \mathrm{E}-05$ \\
\hline elavl3.S & 5.12 & $2,58 \mathrm{E}-27$ \\
\hline barhl2.L & 5.09 & $6,54 \mathrm{E}-10$ \\
\hline phlda1.L & 5.08 & $8,25 \mathrm{E}-10$ \\
\hline bmp3.L & 5.02 & $5,90 \mathrm{E}-11$ \\
\hline nhlh1.S & 5.01 & $4,72 \mathrm{E}-06$ \\
\hline tcf15.S & 5.01 & $2,37 \mathrm{E}-06$ \\
\hline tfap2e.S & 5 & $3,53 \mathrm{E}-15$ \\
\hline nhlh1.L & 4.99 & $8,91 \mathrm{E}-07$ \\
\hline Scrt1.L & 4.97 & $1,49 \mathrm{E}-06$ \\
\hline gpr63.S & 4.96 & $1,94 \mathrm{E}-18$ \\
\hline tuba4a.L & 4.9 & $1,52 \mathrm{E}-28$ \\
\hline st18.L & 4.82 & $1,45 \mathrm{E}-07$ \\
\hline$t \mid x 3 . S$ & 4.8 & $5,65 \mathrm{E}-10$ \\
\hline
\end{tabular}




\begin{tabular}{|c|c|c|}
\hline eln2.L & 4.79 & $5,31 \mathrm{E}-11$ \\
\hline$t / x 3 . L$ & 4.77 & $1,37 \mathrm{E}-14$ \\
\hline tubb3.S & 4.76 & $1,44 \mathrm{E}-16$ \\
\hline dmrta2.L & 4.72 & $4,72 \mathrm{E}-19$ \\
\hline gch1.L & 4.7 & $2,41 \mathrm{E}-37$ \\
\hline map3k12.S & 4.67 & $6,04 \mathrm{E}-09$ \\
\hline pcmtd1.L & 4.66 & 1,26E-05 \\
\hline ak3.S & 4.64 & $1,34 \mathrm{E}-08$ \\
\hline Xelaev18007508m.g & 4.64 & $1,25 E-04$ \\
\hline LOC100490531-like.S & 4.62 & $8,44 \mathrm{E}-05$ \\
\hline fitm1.L & 4.61 & $6,16 \mathrm{E}-05$ \\
\hline tmem116.L & 4.6 & $1,13 \mathrm{E}-20$ \\
\hline adcyap1.S & 4.56 & $2,19 \mathrm{E}-05$ \\
\hline LOC100127750.S & 4.56 & $9,24 \mathrm{E}-08$ \\
\hline Cuzd1.L & 4.55 & $1,01 \mathrm{E}-04$ \\
\hline slc3a2.L & 4.53 & $8,31 \mathrm{E}-15$ \\
\hline unc5a.L & 4.52 & $2,30 \mathrm{E}-06$ \\
\hline Xelaev18021728m.g & 4.52 & $8,11 \mathrm{E}-05$ \\
\hline tcf15.L & 4.49 & $1,79 \mathrm{E}-10$ \\
\hline Xetrov90027705m.S & 4.49 & $1,15 \mathrm{E}-04$ \\
\hline$m m p 17 . L$ & 4.48 & $9,72 \mathrm{E}-07$ \\
\hline gria1-like.1.S & 4.42 & $1,08 \mathrm{E}-07$ \\
\hline skor1.S & 4.42 & $1,32 \mathrm{E}-12$ \\
\hline des.1.S & 4.4 & $1,46 \mathrm{E}-04$ \\
\hline tal1.L & 4.39 & $1,74 \mathrm{E}-04$ \\
\hline tespa1.S & 4.39 & $6,16 \mathrm{E}-05$ \\
\hline sparc. L & 4.38 & $4,17 \mathrm{E}-07$ \\
\hline aqp3.L & 4.37 & $7,14 \mathrm{E}-05$ \\
\hline rassf6.L & 4.34 & $3,33 \mathrm{E}-05$ \\
\hline ptger3.L & 4.32 & $5,67 \mathrm{E}-08$ \\
\hline mcf2l.S & 4.31 & $7,41 \mathrm{E}-10$ \\
\hline slc43a2.S & 4.31 & $2,38 \mathrm{E}-22$ \\
\hline $\operatorname{lh} \times 8 . L$ & 4.3 & $2,85 \mathrm{E}-18$ \\
\hline prdm8.L & 4.29 & $3,03 E-04$ \\
\hline cacnb1.S & 4.28 & $2,58 \mathrm{E}-04$ \\
\hline Xelaev18047032m.g & 4.27 & $7,28 \mathrm{E}-05$ \\
\hline cyp1c1.L & 4.23 & $2,75 \mathrm{E}-04$ \\
\hline six1.S & 4.23 & $1,86 \mathrm{E}-05$ \\
\hline
\end{tabular}




\begin{tabular}{|c|c|c|}
\hline gad1.1.L & 4.2 & $2,45 \mathrm{E}-06$ \\
\hline tfap2e.L & 4.2 & $1,15 \mathrm{E}-06$ \\
\hline emilin1.L & 4.19 & $1,04 \mathrm{E}-10$ \\
\hline bag2.L & 4.16 & $3,76 \mathrm{E}-04$ \\
\hline dpp10.S & 4.15 & 5,57E-07 \\
\hline nat14.S & 4.14 & 5,69E-05 \\
\hline evi5.L & 4.13 & $2,58 \mathrm{E}-30$ \\
\hline snai2.L & 4.13 & $6,74 \mathrm{E}-13$ \\
\hline zc3h12c.S & 4.12 & $4,10 \mathrm{E}-15$ \\
\hline Xelaev18007001m.g & 4.11 & $2,37 \mathrm{E}-20$ \\
\hline edn1.L & 4.06 & 7,53E-07 \\
\hline LOC100487796-like.L & 4.03 & 1,21E-09 \\
\hline$m t c / 1 . S$ & 4.02 & $1,14 \mathrm{E}-05$ \\
\hline Xelaev18006418m.g & 4.02 & $4,59 \mathrm{E}-04$ \\
\hline kank2.L & 4.01 & 1,06E-03 \\
\hline neurog2.S & 3.99 & $4,04 \mathrm{E}-10$ \\
\hline adamts/1.L & 3.9 & $1,11 \mathrm{E}-08$ \\
\hline fezf1.L & 3.89 & $3,74 \mathrm{E}-04$ \\
\hline des.1.L & 3.88 & 7,51E-05 \\
\hline kiaa1755.L & 3.88 & $1,39 \mathrm{E}-04$ \\
\hline LOC100496628-like.L & 3.87 & $6,87 \mathrm{E}-06$ \\
\hline LOC100271753.L & 3.86 & $2,21 \mathrm{E}-06$ \\
\hline barhl2.S & 3.81 & 4,87E-04 \\
\hline runx1t1.S & 3.77 & $5,46 \mathrm{E}-06$ \\
\hline hmox1.L & 3.76 & $3,23 E-28$ \\
\hline LOC100488523.L & 3.76 & $6,38 \mathrm{E}-04$ \\
\hline des.2.L & 3.74 & $3,10 \mathrm{E}-03$ \\
\hline serpinf1.L & 3.74 & $1,45 \mathrm{E}-07$ \\
\hline hmox1.S & 3.73 & $1,48 \mathrm{E}-03$ \\
\hline $\operatorname{drgx} . S$ & 3.72 & $9,95 \mathrm{E}-07$ \\
\hline reep5.S & 3.72 & $1,34 \mathrm{E}-16$ \\
\hline adcyap1.L & 3.69 & $1,50 \mathrm{E}-03$ \\
\hline arhgef26.S & 3.69 & $4,63 \mathrm{E}-05$ \\
\hline $\operatorname{atg} 9 b . L$ & 3.69 & $2,25 \mathrm{E}-11$ \\
\hline cacna2d2.L & 3.68 & $6,27 \mathrm{E}-07$ \\
\hline tmem35.L & 3.68 & $7,24 \mathrm{E}-04$ \\
\hline hes5.2.L & 3.67 & $1,47 \mathrm{E}-06$ \\
\hline kiaa1715.S & 3.66 & 2,54E-03 \\
\hline
\end{tabular}




\begin{tabular}{|c|c|c|}
\hline pou3f2.L & 3.66 & $1,45 \mathrm{E}-07$ \\
\hline hdac7.S & 3.64 & $4,16 \mathrm{E}-05$ \\
\hline dlk2.L & 3.63 & $5,60 \mathrm{E}-05$ \\
\hline bhlhe22.L & 3.59 & $2,83 \mathrm{E}-04$ \\
\hline eva1b.S & 3.59 & $8,10 \mathrm{E}-04$ \\
\hline bcar1.S & 3.58 & $2,98 \mathrm{E}-06$ \\
\hline hoxc5.S & 3.58 & $2,04 \mathrm{E}-03$ \\
\hline Imo2.S & 3.58 & $2,51 \mathrm{E}-03$ \\
\hline samd7.L & 3.58 & 3,35E-05 \\
\hline cbfa2t2.S & 3.57 & $8,07 \mathrm{E}-21$ \\
\hline runx1t1.L & 3.57 & 3,27E-08 \\
\hline zc3h12c.L & 3.56 & $5,45 \mathrm{E}-17$ \\
\hline cbfa2t2.L & 3.55 & $2,37 \mathrm{E}-20$ \\
\hline ror2.S & 3.55 & $8,28 \mathrm{E}-12$ \\
\hline cbfa2t3.L & 3.52 & $2,06 \mathrm{E}-03$ \\
\hline six3.L & 3.52 & $1,08 \mathrm{E}-08$ \\
\hline Imcd1.S & 3.51 & $8,02 \mathrm{E}-12$ \\
\hline chat.S & 3.49 & $4,76 \mathrm{E}-03$ \\
\hline tapbp.L & 3.49 & $3,16 \mathrm{E}-04$ \\
\hline adam33.S & 3.48 & $9,49 \mathrm{E}-06$ \\
\hline zbtb16.S & 3.47 & $7,30 \mathrm{E}-09$ \\
\hline Xetrov90000859m.L & 3.46 & 1,78E-05 \\
\hline ca14.S & 3.41 & 4,67E-05 \\
\hline fdft1.L & 3.39 & $1,01 \mathrm{E}-06$ \\
\hline gas7.L & 3.39 & $2,81 \mathrm{E}-07$ \\
\hline clip2.S & 3.38 & $2,09 \mathrm{E}-06$ \\
\hline kank3.L & 3.38 & 1,87E-08 \\
\hline mespa.S & 3.36 & $7,44 \mathrm{E}-03$ \\
\hline irx3.L & 3.35 & $3,68 \mathrm{E}-05$ \\
\hline rgmb.L & 3.32 & $1,86 \mathrm{E}-11$ \\
\hline skor1.L & 3.32 & $2,52 \mathrm{E}-06$ \\
\hline pak3.S & 3.31 & $6,49 \mathrm{E}-23$ \\
\hline rgs $10 . L$ & 3.31 & $1,95 \mathrm{E}-03$ \\
\hline Xelaev18024617m.g & 3.31 & $2,73 \mathrm{E}-03$ \\
\hline map2.L & 3.3 & $8,94 \mathrm{E}-05$ \\
\hline p2ry4.L & 3.3 & 1,07E-08 \\
\hline Xetrov90010371m.L & 3.3 & $7,61 \mathrm{E}-04$ \\
\hline epb41/1.L & 3.29 & $3,27 \mathrm{E}-04$ \\
\hline
\end{tabular}




\begin{tabular}{|c|c|c|}
\hline foxc2.S & 3.28 & $2,51 \mathrm{E}-03$ \\
\hline sox11.L & 3.28 & $2,79 \mathrm{E}-17$ \\
\hline zcchc24.L & 3.28 & $7,32 \mathrm{E}-03$ \\
\hline adprh.S & 3.27 & $2,26 \mathrm{E}-04$ \\
\hline fam212a.L & 3.26 & $3,93 \mathrm{E}-16$ \\
\hline ppp1r14b.S & 3.26 & 1,19E-05 \\
\hline Xetrov90016928m.L & 3.25 & $9,10 \mathrm{E}-03$ \\
\hline arhgap4.L & 3.24 & $5,60 \mathrm{E}-05$ \\
\hline c8orf46.S & 3.24 & $1,18 \mathrm{E}-03$ \\
\hline dennd2c.L & 3.24 & $6,28 \mathrm{E}-11$ \\
\hline otx2.L & 3.24 & $1,03 \mathrm{E}-02$ \\
\hline pip4k2b.L & 3.24 & $2,08 \mathrm{E}-06$ \\
\hline gfra1.S & 3.2 & $2,29 E-04$ \\
\hline pmp22.L & 3.19 & $7,49 \mathrm{E}-03$ \\
\hline gfi1.L & 3.16 & $2,45 E-04$ \\
\hline plekhg4.S & 3.16 & $6,32 \mathrm{E}-10$ \\
\hline celf2.L & 3.15 & $2,67 \mathrm{E}-04$ \\
\hline ncmap.S & 3.15 & $3,65 \mathrm{E}-05$ \\
\hline cdc42ep3.S & 3.14 & $1,38 \mathrm{E}-04$ \\
\hline$d b n 1 . L$ & 3.14 & $5,50 \mathrm{E}-34$ \\
\hline nrp2.L & 3.14 & $1,25 \mathrm{E}-08$ \\
\hline sfrp2.L & 3.14 & $1,53 \mathrm{E}-11$ \\
\hline$z b t b 18 . S$ & 3.14 & $4,11 \mathrm{E}-09$ \\
\hline rundc3a.L & 3.13 & $7,20 \mathrm{E}-04$ \\
\hline pkdcc.L & 3.12 & $1,06 \mathrm{E}-03$ \\
\hline sox11.S & 3.1 & $7,43 \mathrm{E}-13$ \\
\hline aldh1a2.S & 3.09 & 1,74E-09 \\
\hline dlc.S & 3.09 & $1,20 \mathrm{E}-04$ \\
\hline nhs.S & 3.09 & $4,17 \mathrm{E}-04$ \\
\hline nox4.S & 3.09 & $7,20 \mathrm{E}-03$ \\
\hline faxc.L & 3.08 & $4,03 \mathrm{E}-24$ \\
\hline olig2.S & 3.08 & $5,22 \mathrm{E}-03$ \\
\hline ttc9.L & 3.08 & $5,96 \mathrm{E}-26$ \\
\hline$c p / x 2 . S$ & 3.07 & $2,58 \mathrm{E}-02$ \\
\hline Xelaev18020376m.g & 3.07 & $3,13 \mathrm{E}-02$ \\
\hline ptprt.L & 3.06 & $1,68 \mathrm{E}-04$ \\
\hline plekhg4.L & 3.05 & $8,02 \mathrm{E}-12$ \\
\hline $\operatorname{vim} . S$ & 3.03 & $2,25 \mathrm{E}-07$ \\
\hline
\end{tabular}




\begin{tabular}{|c|c|c|}
\hline$z e b 2 . L$ & 3.03 & $2,50 \mathrm{E}-05$ \\
\hline$d b n 1 . S$ & 3.02 & $3,02 E-15$ \\
\hline dlx6.L & 3.02 & $1,75 \mathrm{E}-07$ \\
\hline clstn3.L & 3.01 & $2,28 \mathrm{E}-02$ \\
\hline$d m b \times 1 . S$ & 3.01 & $2,53 \mathrm{E}-02$ \\
\hline klf7.L & 3.01 & $7,48 \mathrm{E}-04$ \\
\hline LOC100125030.S & 3.01 & $5,74 \mathrm{E}-03$ \\
\hline pla2g12b.S & 3.01 & 6,66E-03 \\
\hline rnf165.L & 3.01 & $2,97 E-11$ \\
\hline Xelaev18021928m.g & 3.01 & $1,96 \mathrm{E}-15$ \\
\hline LOC100492460.L & 2.99 & $1,91 \mathrm{E}-02$ \\
\hline s1pr5.S & 2.99 & $4,22 \mathrm{E}-11$ \\
\hline slc38a8.L & 2.99 & $1,08 \mathrm{E}-03$ \\
\hline st8sia6.L & 2.99 & $5,41 \mathrm{E}-07$ \\
\hline ap/nr.L & 2.98 & $1,39 \mathrm{E}-06$ \\
\hline aplnr.S & 2.98 & $2,67 E-05$ \\
\hline$c p / x 2 . L$ & 2.98 & $9,09 \mathrm{E}-04$ \\
\hline crmp1.S & 2.98 & $9,73 \mathrm{E}-04$ \\
\hline sybu.L & 2.98 & $8,46 \mathrm{E}-08$ \\
\hline$a b t b 2 . S$ & 2.97 & $2,39 E-03$ \\
\hline shox2.L & 2.96 & 3,59E-02 \\
\hline neurog1.L & 2.95 & $1,62 \mathrm{E}-02$ \\
\hline Xelaev18041660m.g & 2.95 & $1,58 \mathrm{E}-02$ \\
\hline cpe.S & 2.94 & $1,00 \mathrm{E}-02$ \\
\hline$e m \times 1 . L$ & 2.94 & $1,30 \mathrm{E}-02$ \\
\hline slc25a38.L & 2.94 & $1,32 \mathrm{E}-08$ \\
\hline zic3.L & 2.94 & 1,31E-02 \\
\hline cables1.L & 2.93 & 6,37E-03 \\
\hline Xelaev18003350m.g & 2.93 & $2,93 \mathrm{E}-02$ \\
\hline$a 2 m . S$ & 2.92 & $5,63 \mathrm{E}-27$ \\
\hline slc8a2.S & 2.91 & $1,83 \mathrm{E}-02$ \\
\hline ulk1.L & 2.91 & $7,25 \mathrm{E}-06$ \\
\hline Xelaev18016582m.g & 2.91 & $1,42 \mathrm{E}-04$ \\
\hline Xelaev18047968m.g & 2.91 & $2,16 \mathrm{E}-02$ \\
\hline arid1b.L & 2.9 & $4,23 \mathrm{E}-19$ \\
\hline cacna2d1.L & 2.9 & $2,18 \mathrm{E}-09$ \\
\hline Xelaev18030957m.g & 2.9 & $1,80 \mathrm{E}-02$ \\
\hline il5ra.S & 2.88 & $2,11 \mathrm{E}-04$ \\
\hline
\end{tabular}




\begin{tabular}{|c|c|c|}
\hline LOC100487395.S & 2.88 & $4,17 E-04$ \\
\hline LOC101733102.L & 2.88 & 5,99E-04 \\
\hline st18.S & 2.88 & $2,11 \mathrm{E}-02$ \\
\hline zfh $x 4 . S$ & 2.88 & $1,32 \mathrm{E}-06$ \\
\hline cep85I.S & 2.87 & $5,02 \mathrm{E}-10$ \\
\hline nova2.S & 2.87 & $1,27 \mathrm{E}-03$ \\
\hline plekhd1.L & 2.87 & $3,27 E-02$ \\
\hline hlf.L & 2.86 & $2,53 E-03$ \\
\hline pzp.L & 2.86 & $2,17 \mathrm{E}-04$ \\
\hline$a s b 2 . L$ & 2.85 & $3,87 \mathrm{E}-02$ \\
\hline bhlhe40.L & 2.85 & $3,94 \mathrm{E}-04$ \\
\hline pdia2.S & 2.85 & $6,64 \mathrm{E}-03$ \\
\hline Xelaev18039965m.g & 2.85 & $2,18 \mathrm{E}-06$ \\
\hline$d b n d d 1 . L$ & 2.84 & $2,95 \mathrm{E}-02$ \\
\hline LOC100494987-like.L & 2.84 & $2,62 \mathrm{E}-02$ \\
\hline ankrd6.L & 2.83 & $5,04 \mathrm{E}-03$ \\
\hline elav/3.L & 2.82 & $4,21 \mathrm{E}-10$ \\
\hline hoxd1.L & 2.82 & $1,14 \mathrm{E}-05$ \\
\hline $\operatorname{limd2.L}$ & 2.82 & $2,04 \mathrm{E}-04$ \\
\hline LOC100485834.L & 2.82 & $1,75 \mathrm{E}-03$ \\
\hline LOC100496678.L & 2.81 & $4,38 \mathrm{E}-02$ \\
\hline rap1gap.S & 2.81 & $2,53 \mathrm{E}-05$ \\
\hline slc26a9.S & 2.81 & $9,22 \mathrm{E}-03$ \\
\hline Xelaev18038420m.g & 2.81 & $7,03 \mathrm{E}-09$ \\
\hline afap1/1.L & 2.8 & $3,50 \mathrm{E}-04$ \\
\hline$m d k \cdot L$ & 2.8 & $1,45 \mathrm{E}-19$ \\
\hline plod2.S & 2.8 & $7,29 \mathrm{E}-05$ \\
\hline Six1.L & 2.8 & $3,80 \mathrm{E}-03$ \\
\hline asb12.L & 2.79 & $2,95 \mathrm{E}-03$ \\
\hline $\operatorname{lh} x 1 . S$ & 2.79 & $8,75 \mathrm{E}-05$ \\
\hline fam102a.S & 2.78 & $2,35 \mathrm{E}-04$ \\
\hline mamdc2.L & 2.78 & $2,18 \mathrm{E}-03$ \\
\hline pcdh9.L & 2.78 & $1,69 \mathrm{E}-04$ \\
\hline plekhg1.L & 2.78 & $6,74 \mathrm{E}-10$ \\
\hline arhgef26.L & 2.77 & $6,86 \mathrm{E}-05$ \\
\hline btbd19.L & 2.77 & $4,27 \mathrm{E}-02$ \\
\hline gcnt1.S & 2.77 & $7,64 \mathrm{E}-05$ \\
\hline kcna1.S & 2.77 & 8,53E-04 \\
\hline
\end{tabular}




\begin{tabular}{|c|c|c|}
\hline draxin.S & 2.76 & 1,90E-02 \\
\hline nuak1.L & 2.76 & $9,87 E-06$ \\
\hline cacnb3.L & 2.75 & $3,23 \mathrm{E}-02$ \\
\hline$m s \times 2 . S$ & 2.75 & $1,86 \mathrm{E}-08$ \\
\hline hcar3.L & 2.74 & $1,05 E-03$ \\
\hline hes5.2.S & 2.74 & $4,75 \mathrm{E}-03$ \\
\hline ag1.S & 2.73 & $3,03 E-08$ \\
\hline CXCr2.L & 2.73 & $4,90 \mathrm{E}-02$ \\
\hline dnajc6.S & 2.73 & $9,28 \mathrm{E}-03$ \\
\hline hes6.2.L & 2.71 & $3,04 \mathrm{E}-02$ \\
\hline tmem169.L & 2.7 & $4,11 \mathrm{E}-09$ \\
\hline$a 2 m \cdot L$ & 2.69 & $2,93 \mathrm{E}-04$ \\
\hline cldn5.L & 2.69 & $5,81 \mathrm{E}-03$ \\
\hline map3k13.L & 2.69 & $2,23 \mathrm{E}-02$ \\
\hline$s p 5 . L$ & 2.68 & $2,48 \mathrm{E}-06$ \\
\hline tet3.S & 2.68 & $6,20 \mathrm{E}-04$ \\
\hline glis2.S & 2.67 & $1,07 E-05$ \\
\hline Xelaev18043580m.g & 2.67 & $1,34 \mathrm{E}-08$ \\
\hline insm1.L & 2.66 & $4,72 \mathrm{E}-06$ \\
\hline pcdh18.L & 2.66 & $3,39 E-05$ \\
\hline tie1.S & 2.66 & 1,77E-02 \\
\hline auts2.S & 2.65 & $1,39 \mathrm{E}-11$ \\
\hline cited2.L & 2.65 & $7,77 \mathrm{E}-12$ \\
\hline faxc.S & 2.65 & $2,50 \mathrm{E}-02$ \\
\hline agbl1.S & 2.64 & $5,80 \mathrm{E}-08$ \\
\hline agbl1.S & 2.64 & $5,80 \mathrm{E}-08$ \\
\hline agbl1.S & 2.64 & $5,80 \mathrm{E}-08$ \\
\hline agbl1.S & 2.64 & $5,80 \mathrm{E}-08$ \\
\hline LOC100495335.L & 2.64 & $5,69 \mathrm{E}-03$ \\
\hline limd2.S & 2.63 & $5,21 \mathrm{E}-06$ \\
\hline dmrta2.S & 2.62 & $3,39 \mathrm{E}-05$ \\
\hline fam89a.S & 2.62 & $9,85 \mathrm{E}-06$ \\
\hline grb14.L & 2.62 & $2,91 \mathrm{E}-02$ \\
\hline hes5_X2.L & 2.59 & $3,16 \mathrm{E}-06$ \\
\hline Irp4.S & 2.59 & $2,09 E-06$ \\
\hline tdrp.L & 2.58 & $3,01 \mathrm{E}-20$ \\
\hline Irrn1-like.1.S & 2.57 & $3,15 \mathrm{E}-11$ \\
\hline nes.L & 2.57 & $6,27 \mathrm{E}-07$ \\
\hline
\end{tabular}




\begin{tabular}{|c|c|c|}
\hline cel-like.1.S & 2.56 & $7,17 \mathrm{E}-03$ \\
\hline ctsk.L & 2.56 & $2,32 \mathrm{E}-03$ \\
\hline irx4.L & 2.56 & $2,60 \mathrm{E}-02$ \\
\hline $\operatorname{lh} \times 5 . L$ & 2.55 & $9,53 \mathrm{E}-18$ \\
\hline LOC100489305.S & 2.55 & $9,69 \mathrm{E}-03$ \\
\hline fst/1.L & 2.54 & $3,73 E-02$ \\
\hline krt19.S & 2.53 & $2,73 \mathrm{E}-12$ \\
\hline LOC101733157.L & 2.53 & $3,16 \mathrm{E}-04$ \\
\hline Xelaev18016676m.g & 2.53 & $5,12 \mathrm{E}-08$ \\
\hline$a b t b 2 . L$ & 2.52 & $2,05 \mathrm{E}-03$ \\
\hline pcmtd1.S & 2.52 & $8,75 \mathrm{E}-05$ \\
\hline f2r.S & 2.51 & 1,65E-02 \\
\hline st3gal2.1.L & 2.51 & $4,22 \mathrm{E}-05$ \\
\hline$a b c b 9 . L$ & 2.5 & $2,97 \mathrm{E}-19$ \\
\hline eya1.L & 2.5 & $2,87 \mathrm{E}-04$ \\
\hline fam102a.L & 2.5 & $7,55 \mathrm{E}-05$ \\
\hline frmd6.L & 2.49 & $8,44 \mathrm{E}-18$ \\
\hline gas1.L & 2.49 & $2,19 \mathrm{E}-05$ \\
\hline Xelaev18023424m.g & 2.49 & $2,66 \mathrm{E}-02$ \\
\hline Xelaev18039076m.g & 2.49 & $4,64 \mathrm{E}-03$ \\
\hline arhgap33.S & 2.48 & $4,91 \mathrm{E}-05$ \\
\hline hes5.3.S & 2.48 & $9,01 \mathrm{E}-07$ \\
\hline srrm4.L & 2.48 & $1,50 \mathrm{E}-03$ \\
\hline egr4.L & 2.47 & $4,01 \mathrm{E}-02$ \\
\hline fgf16.L & 2.47 & $6,40 \mathrm{E}-03$ \\
\hline hes2.L & 2.47 & $1,34 \mathrm{E}-05$ \\
\hline pnhd.S & 2.47 & $2,56 \mathrm{E}-02$ \\
\hline kans/1I.L & 2.45 & $1,11 \mathrm{E}-02$ \\
\hline rnf165.S & 2.45 & $3,41 \mathrm{E}-07$ \\
\hline Xelaev18024498m.g & 2.45 & $3,42 \mathrm{E}-02$ \\
\hline hand2.S & 2.44 & $3,54 \mathrm{E}-04$ \\
\hline stk40.L & 2.44 & $2,95 \mathrm{E}-10$ \\
\hline tox3.L & 2.44 & $3,41 \mathrm{E}-12$ \\
\hline f3.S & 2.43 & $2,91 \mathrm{E}-03$ \\
\hline rfk.S & 2.43 & $2,03 E-03$ \\
\hline Xelaev18019210m.g & 2.43 & $6,18 \mathrm{E}-03$ \\
\hline prdm14.L & 2.42 & $2,33 \mathrm{E}-05$ \\
\hline rnd3.S & 2.42 & 4,59E-05 \\
\hline
\end{tabular}




\begin{tabular}{|c|c|c|}
\hline tuba4a.S & 2.41 & $8,16 \mathrm{E}-05$ \\
\hline fgd1.S & 2.4 & 5,75E-03 \\
\hline irx1.L & 2.4 & $6,18 \mathrm{E}-03$ \\
\hline zfhx4.L & 2.4 & $7,52 \mathrm{E}-04$ \\
\hline LOC100496628-like.S & 2.39 & $4,59 E-02$ \\
\hline klh/25.S & 2.38 & $2,12 \mathrm{E}-03$ \\
\hline cemip.S & 2.37 & $4,46 \mathrm{E}-04$ \\
\hline mdk.S & 2.37 & 5,63E-05 \\
\hline slc43a2.L & 2.37 & 1,01E-03 \\
\hline myt1.L & 2.36 & $6,16 \mathrm{E}-07$ \\
\hline Xelaev18009525m.g & 2.36 & $6,38 \mathrm{E}-04$ \\
\hline eya2.S & 2.35 & $4,76 \mathrm{E}-03$ \\
\hline neurog2.L & 2.35 & $4,08 \mathrm{E}-02$ \\
\hline slit1.S & 2.35 & $2,52 \mathrm{E}-03$ \\
\hline znf329.S & 2.35 & $5,74 \mathrm{E}-04$ \\
\hline aldh1a2.L & 2.34 & 1,17E-07 \\
\hline cep85I.L & 2.33 & $5,64 \mathrm{E}-07$ \\
\hline hoxd1.S & 2.33 & 4,77E-02 \\
\hline Irig1.S & 2.33 & 2,27E-02 \\
\hline myt1.S & 2.33 & $1,74 \mathrm{E}-04$ \\
\hline ndst1.S & 2.33 & $7,54 \mathrm{E}-07$ \\
\hline irf1.L & 2.32 & $3,64 \mathrm{E}-06$ \\
\hline pitx3.L & 2.32 & $2,33 E-02$ \\
\hline spo11.L & 2.32 & $3,16 \mathrm{E}-02$ \\
\hline btg2.S & 2.31 & $6,38 \mathrm{E}-04$ \\
\hline mctp2.L & 2.31 & $3,76 \mathrm{E}-03$ \\
\hline LOC100492804.L & 2.3 & 3,65E-05 \\
\hline manea.L & 2.3 & 3,97E-03 \\
\hline$m t c / 1 . L$ & 2.3 & $1,47 \mathrm{E}-03$ \\
\hline b4galnt1.S & 2.29 & $2,87 \mathrm{E}-04$ \\
\hline cldn6.1.L & 2.29 & $2,17 \mathrm{E}-08$ \\
\hline Xelaev18028850m.g & 2.29 & $5,93 E-05$ \\
\hline cpe.L & 2.28 & $6,64 \mathrm{E}-06$ \\
\hline$z e b 2 . S$ & 2.28 & $6,74 \mathrm{E}-04$ \\
\hline dennd2c.S & 2.27 & 3,29E-05 \\
\hline hes5_X2.S & 2.27 & $1,05 \mathrm{E}-03$ \\
\hline smoc1.S & 2.27 & $2,48 \mathrm{E}-02$ \\
\hline arhgap36.L & 2.26 & $9,88 \mathrm{E}-04$ \\
\hline
\end{tabular}




\begin{tabular}{|c|c|c|}
\hline ephb3.S & 2.26 & $1,11 \mathrm{E}-10$ \\
\hline smim3.S & 2.25 & 2,93E-03 \\
\hline Xelaev18033094m.g & 2.25 & $3,86 \mathrm{E}-02$ \\
\hline cdc42ep4.L & 2.23 & $2,79 \mathrm{E}-03$ \\
\hline tmem74b.S & 2.23 & $8,06 \mathrm{E}-03$ \\
\hline Xelaev18042973m.g & 2.23 & 4,99E-02 \\
\hline meis1.L & 2.22 & 6,77E-05 \\
\hline rufy3.L & 2.22 & 4,54E-06 \\
\hline gtf2ird1.L & 2.21 & 1,94E-02 \\
\hline rasal2-like.2.S & 2.21 & $8,02 \mathrm{E}-12$ \\
\hline antxr2.L & 2.2 & $7,09 \mathrm{E}-04$ \\
\hline rab3a.S & 2.2 & $1,19 \mathrm{E}-12$ \\
\hline mcc.S & 2.19 & $2,69 \mathrm{E}-03$ \\
\hline mybl1.S & 2.19 & 1,07E-03 \\
\hline tuba1b.L & 2.19 & 9,54E-07 \\
\hline cdyl2.S & 2.18 & $1,74 \mathrm{E}-02$ \\
\hline gfi1.S & 2.18 & 9,57E-03 \\
\hline LOC100488626.L & 2.18 & $1,96 \mathrm{E}-02$ \\
\hline $\operatorname{vim} . L$ & 2.18 & $1,13 \mathrm{E}-06$ \\
\hline acy3.L & 2.17 & 9,06E-07 \\
\hline numbl.L & 2.17 & $3,10 \mathrm{E}-07$ \\
\hline ppp1r1a.L & 2.17 & $2,00 \mathrm{E}-04$ \\
\hline rgma.L & 2.17 & $7,29 \mathrm{E}-07$ \\
\hline dgki.S & 2.16 & $2,10 \mathrm{E}-02$ \\
\hline insm2.L & 2.15 & $4,46 \mathrm{E}-02$ \\
\hline krt18.S & 2.15 & $6,89 \mathrm{E}-07$ \\
\hline cuedc1.S & 2.14 & $1,06 \mathrm{E}-11$ \\
\hline $\ln x 1 . L$ & 2.14 & $4,68 \mathrm{E}-02$ \\
\hline ptch2.S & 2.14 & $2,65 \mathrm{E}-02$ \\
\hline rph3al.S & 2.14 & $2,13 \mathrm{E}-02$ \\
\hline tuft1.L & 2.14 & $3,29 \mathrm{E}-02$ \\
\hline Xelaev18016674m.g & 2.14 & $8,87 \mathrm{E}-04$ \\
\hline dact1.L & 2.13 & $9,63 \mathrm{E}-13$ \\
\hline fsd1.L & 2.13 & $2,13 \mathrm{E}-02$ \\
\hline foxc1.S & 2.12 & $3,99 \mathrm{E}-02$ \\
\hline mcf2l.2.L & 2.12 & $6,42 \mathrm{E}-03$ \\
\hline pak3.L & 2.11 & $1,84 \mathrm{E}-03$ \\
\hline sall3.L & 2.11 & 1,87E-08 \\
\hline
\end{tabular}




\begin{tabular}{|c|c|c|}
\hline aim1I.S & 2.1 & 1,15E-02 \\
\hline dlk2.S & 2.1 & $1,80 \mathrm{E}-02$ \\
\hline hes8.L & 2.1 & $8,78 \mathrm{E}-05$ \\
\hline dnajc6.L & 2.09 & $2,00 \mathrm{E}-02$ \\
\hline cdc42se2-like.1.L & 2.08 & 1,29E-05 \\
\hline dclk1.S & 2.08 & $3,44 \mathrm{E}-02$ \\
\hline insm1.S & 2.08 & $3,83 \mathrm{E}-03$ \\
\hline tmem170b.L & 2.08 & $3,64 \mathrm{E}-03$ \\
\hline kctd1.L & 2.07 & $2,42 \mathrm{E}-03$ \\
\hline map1/c3a.L & 2.07 & $1,95 \mathrm{E}-03$ \\
\hline nckap5I.L & 2.06 & $3,82 \mathrm{E}-05$ \\
\hline$f b \times 032 . L$ & 2.05 & $2,16 \mathrm{E}-02$ \\
\hline pik3r3.L & 2.05 & $2,54 \mathrm{E}-04$ \\
\hline rbm38.L & 2.05 & 1,74E-09 \\
\hline rexo1.s & 2.05 & $4,59 \mathrm{E}-05$ \\
\hline rnf130.S & 2.05 & $2,99 \mathrm{E}-05$ \\
\hline kiaa1468.L & 2.04 & $1,08 \mathrm{E}-03$ \\
\hline ras/10b.L & 2.04 & 1,09E-06 \\
\hline Xelaev18044942m.g & 2.04 & $1,75 \mathrm{E}-04$ \\
\hline tes.L & 2.03 & $4,43 \mathrm{E}-03$ \\
\hline cttnbp2nl.L & 2.02 & $7,68 \mathrm{E}-04$ \\
\hline scamp4.S & 2.02 & $4,56 \mathrm{E}-02$ \\
\hline sfrp2.S & 2.02 & $1,01 \mathrm{E}-14$ \\
\hline stmn1.S & 2.02 & $2,81 \mathrm{E}-08$ \\
\hline hoxc4.S & 2.01 & $2,06 \mathrm{E}-02$ \\
\hline fstl1.S & 2 & $1,39 \mathrm{E}-02$ \\
\hline LOC100496433.1.L & 2 & $1,68 \mathrm{E}-04$ \\
\hline Irp4.L & 2 & $4,06 \mathrm{E}-06$ \\
\hline pmp22.S & 2 & $1,78 \mathrm{E}-02$ \\
\hline
\end{tabular}

\subsubsection{Candidate gene list for the RNA sequencing analysis of Brg1}

\section{knock-down experiment}

Given are the genes which are differentially expressed between Ptf1a-GR + cMO or Ptf1a-GR + Brg1MO overexpressing animal caps over non-injected control caps in two individual replicates. Given are the gene ID, the $\log _{2} \mathrm{FC}$ activation over $\mathrm{CC}$ and the $p$-value. 
Table S18: Summary of differentially expressed genes by Ptf1a + cMO

\begin{tabular}{|c|c|c|}
\hline ID & log2FC & P-value \\
\hline Xelaev18007508m.g & 14.26 & $9.14 \mathrm{E}-18$ \\
\hline neurod4.L & 12.86 & $4.40 \mathrm{E}-14$ \\
\hline prdm13.L & 12.29 & $4.88 \mathrm{E}-14$ \\
\hline prph.S & 12.22 & $6.31 \mathrm{E}-28$ \\
\hline neurod4.S & 11.98 & $1.88 \mathrm{E}-11$ \\
\hline nhlh1.L & 11.94 & $4.57 \mathrm{E}-13$ \\
\hline pou3f1.L & 11.65 & $1.21 \mathrm{E}-11$ \\
\hline prdm13.S & 11.46 & $7.21 \mathrm{E}-19$ \\
\hline nhlh1.S & 11.42 & $1.87 \mathrm{E}-11$ \\
\hline scrt1.S & 11.25 & $3.90 \mathrm{E}-12$ \\
\hline igfbp/1.L & 11.16 & $7.42 \mathrm{E}-11$ \\
\hline dpys/4.L & 10.93 & $6.15 \mathrm{E}-10$ \\
\hline st18.L & 10.78 & $2.33 \mathrm{E}-10$ \\
\hline neurog1.L & 10.75 & $1.53 \mathrm{E}-10$ \\
\hline des.2.L & 10.49 & $1.78 \mathrm{E}-08$ \\
\hline hoxc3.L & 10.43 & 4.64E-07 \\
\hline prdm8.L & 10.31 & $2.43 \mathrm{E}-04$ \\
\hline Imo2.S & 10.28 & $4.37 \mathrm{E}-09$ \\
\hline Ibx1.S & 10.14 & $1.36 \mathrm{E}-08$ \\
\hline LOC100490531-like.L & 9.95 & 3.31E-09 \\
\hline LOC100496628-like.L & 9.89 & $1.83 \mathrm{E}-07$ \\
\hline pyy.L & 9.88 & $1.72 \mathrm{E}-07$ \\
\hline hes5.2.L & 9.75 & 7.77E-07 \\
\hline aqp3.L & 9.69 & $1.68 \mathrm{E}-04$ \\
\hline$e b f 2 . L$ & 9.65 & 6.74E-32 \\
\hline fezf1.L & 9.64 & $1.12 \mathrm{E}-05$ \\
\hline Xetrov90027705m.L & 9.54 & $8.64 \mathrm{E}-15$ \\
\hline nr5a2.L & 9.43 & $1.86 \mathrm{E}-06$ \\
\hline skor1.S & 9.36 & $6.12 \mathrm{E}-12$ \\
\hline Xelaev18047032m.g & 9.35 & $8.25 \mathrm{E}-05$ \\
\hline kiaa1755.L & 9.26 & $4.74 \mathrm{E}-21$ \\
\hline adcyap1.S & 9.19 & $3.14 \mathrm{E}-06$ \\
\hline aldh1b1.L & 9.13 & $6.83 \mathrm{E}-06$ \\
\hline Xelaev18032183m.g & 9.06 & $1.65 \mathrm{E}-06$ \\
\hline mllt11.S & 9.01 & $2.31 \mathrm{E}-06$ \\
\hline tfap2b.S & 9.01 & 8.87E-36 \\
\hline
\end{tabular}




\begin{tabular}{|c|c|c|}
\hline skor2.S & 8.94 & $6.35 \mathrm{E}-04$ \\
\hline pcdh9.L & 8.92 & 8.63E-06 \\
\hline map3k13.L & 8.87 & $9.24 \mathrm{E}-05$ \\
\hline Xelaev18002612m.g & 8.83 & $1.62 E-03$ \\
\hline Xelaev18018357m.g & 8.82 & $1.44 \mathrm{E}-05$ \\
\hline Xelaev18004302m.g & 8.81 & 1.49E-05 \\
\hline tespa1.S & 8.77 & $1.14 \mathrm{E}-05$ \\
\hline shox2.L & 8.76 & $9.85 \mathrm{E}-06$ \\
\hline prdm8.S & 8.73 & $1.70 \mathrm{E}-04$ \\
\hline igfbpl1.S & 8.73 & 5.92E-05 \\
\hline tubb3.L & 8.70 & $7.68 \mathrm{E}-07$ \\
\hline shd.L & 8.64 & $5.11 \mathrm{E}-12$ \\
\hline hesx1.S & 8.56 & 1.26E-09 \\
\hline des.1.L & 8.52 & $2.53 \mathrm{E}-10$ \\
\hline foxd2.L & 8.48 & $7.82 \mathrm{E}-05$ \\
\hline tfap2e.L & 8.35 & $4.09 \mathrm{E}-06$ \\
\hline ebf2.S & 8.34 & $8.84 \mathrm{E}-31$ \\
\hline dpys/3.L & 8.27 & $5.82 \mathrm{E}-79$ \\
\hline nova2.S & 8.27 & $9.24 \mathrm{E}-05$ \\
\hline nes.L & 8.27 & $1.68 \mathrm{E}-19$ \\
\hline pou3f1.S & 8.20 & 8.52E-03 \\
\hline Xelaev18000445m.g & 8.19 & $5.78 \mathrm{E}-05$ \\
\hline tcf15.S & 8.14 & $2.74 \mathrm{E}-04$ \\
\hline Xetrov90027705m.S & 8.14 & $8.38 \mathrm{E}-05$ \\
\hline barhl2.S & 8.03 & $7.62 \mathrm{E}-09$ \\
\hline$n t s . L$ & 8.03 & $1.67 \mathrm{E}-03$ \\
\hline$n k x 3-2 . S$ & 8.01 & $5.50 \mathrm{E}-03$ \\
\hline foxb1.S & 7.99 & $2.18 \mathrm{E}-03$ \\
\hline ptger3.L & 7.99 & $1.32 \mathrm{E}-04$ \\
\hline$h m \times 1.1$ & 7.97 & $5.85 \mathrm{E}-03$ \\
\hline clstn3.L & 7.93 & $2.51 \mathrm{E}-12$ \\
\hline hes6.2.L & 7.92 & $5.89 \mathrm{E}-03$ \\
\hline LOC100490531-like.S & 7.91 & $4.37 \mathrm{E}-04$ \\
\hline sybu.L & 7.89 & $6.70 \mathrm{E}-08$ \\
\hline gad1.1.L & 7.87 & $1.29 \mathrm{E}-16$ \\
\hline ptf1a.L & 7.83 & 6.09E-05 \\
\hline slc26a9.S & 7.83 & $1.28 \mathrm{E}-04$ \\
\hline spry4.S & 7.74 & 1.96E-03 \\
\hline
\end{tabular}




\begin{tabular}{|c|c|c|}
\hline pnhd.S & 7.72 & $1.88 \mathrm{E}-04$ \\
\hline Xelaev18047333m.g & 7.71 & 6.45E-05 \\
\hline eva1b.S & 7.70 & 7.41E-05 \\
\hline bgn.L & 7.68 & $3.14 \mathrm{E}-45$ \\
\hline fam212b.L & 7.66 & $6.98 \mathrm{E}-04$ \\
\hline Xelaev18040877m.g & 7.63 & $5.34 \mathrm{E}-18$ \\
\hline Xelaev18045246m.g & 7.62 & 7.76E-04 \\
\hline map2.S & 7.58 & 8.87E-36 \\
\hline$v s \times 1 . S$ & 7.56 & $6.56 \mathrm{E}-04$ \\
\hline tal1.L & 7.54 & $8.62 \mathrm{E}-08$ \\
\hline stk32a.L & 7.48 & 3.78E-04 \\
\hline map3k12.S & 7.47 & $3.14 \mathrm{E}-07$ \\
\hline Xelaev18036651m.g & 7.46 & $1.23 \mathrm{E}-35$ \\
\hline Xelaev18001525m.g & 7.44 & $1.89 \mathrm{E}-02$ \\
\hline$n p t x 2 . L$ & 7.44 & 1.05E-02 \\
\hline$m m p 17 . L$ & 7.43 & $2.71 \mathrm{E}-04$ \\
\hline chst15.L & 7.41 & $3.38 \mathrm{E}-03$ \\
\hline elavl3.S & 7.41 & $2.06 \mathrm{E}-04$ \\
\hline barhl2.L & 7.40 & $4.55 \mathrm{E}-04$ \\
\hline$m y l 1 . L$ & 7.36 & $2.56 \mathrm{E}-04$ \\
\hline LOC100489771.L & 7.35 & $3.58 \mathrm{E}-03$ \\
\hline tmem116.L & 7.34 & $2.77 \mathrm{E}-17$ \\
\hline slc1a2.S & 7.33 & $4.01 \mathrm{E}-02$ \\
\hline pdyn.S & 7.33 & $1.24 \mathrm{E}-02$ \\
\hline tubb3.S & 7.33 & $6.66 \mathrm{E}-37$ \\
\hline pcdh8.S & 7.33 & $3.38 \mathrm{E}-04$ \\
\hline neurod1.S & 7.32 & $4.31 \mathrm{E}-03$ \\
\hline kirrel2.L & 7.24 & $2.18 \mathrm{E}-81$ \\
\hline chat.S & 7.23 & $3.03 \mathrm{E}-03$ \\
\hline Irrn1-like.1.S & 7.18 & $1.15 \mathrm{E}-45$ \\
\hline kiaa1715.S & 7.17 & $6.42 \mathrm{E}-03$ \\
\hline foxd3.L & 7.17 & $3.60 \mathrm{E}-05$ \\
\hline dmrta2.L & 7.14 & 1.77E-04 \\
\hline$z e b 2 . L$ & 7.14 & $1.44 \mathrm{E}-25$ \\
\hline olig3.S & 7.11 & $6.49 \mathrm{E}-03$ \\
\hline plekhd1.L & 7.11 & $7.85 \mathrm{E}-04$ \\
\hline spns2.L & 7.09 & $7.42 \mathrm{E}-04$ \\
\hline dmrta1.L & 7.05 & 5.53E-03 \\
\hline
\end{tabular}




\begin{tabular}{|c|c|c|}
\hline nat14.S & 7.05 & $2.20 \mathrm{E}-03$ \\
\hline dpp10.S & 7.01 & $2.55 \mathrm{E}-28$ \\
\hline ppp1r9b.S & 6.97 & $2.20 \mathrm{E}-06$ \\
\hline ppp1r9b.L & 6.96 & $3.46 \mathrm{E}-11$ \\
\hline myo3b.L & 6.96 & $6.33 \mathrm{E}-03$ \\
\hline nes.S & 6.95 & $3.22 E-24$ \\
\hline cdh1.L & 6.95 & $6.54 \mathrm{E}-12$ \\
\hline Xelaev18042973m.g & 6.94 & $1.05 \mathrm{E}-05$ \\
\hline paqr9.L & 6.94 & $1.23 \mathrm{E}-08$ \\
\hline neurod1.L & 6.93 & $1.11 \mathrm{E}-02$ \\
\hline$z e b 2 . S$ & 6.93 & $8.19 \mathrm{E}-27$ \\
\hline LOC101732256-like.L & 6.92 & $4.00 \mathrm{E}-02$ \\
\hline Xelaev18033867m.g & 6.91 & 7.82E-03 \\
\hline cacnb1.S & 6.89 & 7.51E-06 \\
\hline Xetrov90008914m.L & 6.88 & $2.01 \mathrm{E}-02$ \\
\hline Xelaev18004376m.g & 6.87 & $4.33 \mathrm{E}-02$ \\
\hline hes5.2.S & 6.84 & $1.05 \mathrm{E}-02$ \\
\hline chst3.L & 6.84 & $2.61 \mathrm{E}-13$ \\
\hline pnhd.L & 6.83 & $2.05 \mathrm{E}-07$ \\
\hline Xelaev18026149m.g & 6.83 & $3.58 \mathrm{E}-03$ \\
\hline shox2.S & 6.75 & $1.51 \mathrm{E}-03$ \\
\hline hoxd1.S & 6.75 & $5.72 \mathrm{E}-03$ \\
\hline myod1.S & 6.74 & $1.51 \mathrm{E}-03$ \\
\hline tal1.S & 6.69 & $4.31 \mathrm{E}-06$ \\
\hline ror2.S & 6.67 & $2.53 \mathrm{E}-21$ \\
\hline kirrel2.S & 6.66 & $1.22 \mathrm{E}-64$ \\
\hline$m m p 28 . L$ & 6.62 & $2.56 \mathrm{E}-02$ \\
\hline slc18a3.S & 6.61 & $1.19 \mathrm{E}-03$ \\
\hline chrd.S & 6.60 & 9.99E-04 \\
\hline Xelaev18022024m.g & 6.59 & $2.08 \mathrm{E}-05$ \\
\hline nova1.L & 6.55 & $9.18 \mathrm{E}-18$ \\
\hline $\ln \times 1 . S$ & 6.49 & 1.89E-02 \\
\hline LOC100127750.S & 6.45 & $2.00 \mathrm{E}-02$ \\
\hline slc24a2.L & 6.41 & $1.93 \mathrm{E}-02$ \\
\hline sp5.S & 6.40 & $2.75 \mathrm{E}-02$ \\
\hline$m n \times 1 . L$ & 6.39 & $1.70 \mathrm{E}-02$ \\
\hline olig2.S & 6.34 & $1.49 \mathrm{E}-20$ \\
\hline Xelaev18046363m.g & 6.33 & $3.12 \mathrm{E}-02$ \\
\hline
\end{tabular}




\begin{tabular}{|c|c|c|}
\hline Xelaev18020334m.g & 6.31 & 3.76E-03 \\
\hline phlda1.L & 6.29 & $5.18 \mathrm{E}-08$ \\
\hline Xelaev18002611m.g & 6.25 & $4.86 \mathrm{E}-05$ \\
\hline LOC100487395.L & 6.23 & $3.28 \mathrm{E}-34$ \\
\hline LOC100487395.S & 6.23 & $2.25 \mathrm{E}-12$ \\
\hline tapbp.L & 6.22 & $3.18 \mathrm{E}-03$ \\
\hline faxc.S & 6.21 & $7.27 \mathrm{E}-05$ \\
\hline hes5_X2.L & 6.21 & $9.17 \mathrm{E}-11$ \\
\hline pax2.L & 6.19 & $2.92 \mathrm{E}-07$ \\
\hline elavl4.L & 6.18 & $3.06 \mathrm{E}-10$ \\
\hline$d m b \times 1 . L$ & 6.17 & $9.20 \mathrm{E}-08$ \\
\hline onecut1.2.L & 6.16 & $1.74 \mathrm{E}-19$ \\
\hline slc32a1.S & 6.16 & $9.89 \mathrm{E}-03$ \\
\hline LOC100490819.L & 6.15 & $2.61 \mathrm{E}-02$ \\
\hline mxra7.L & 6.15 & $3.76 \mathrm{E}-23$ \\
\hline tub.L & 6.08 & $8.56 \mathrm{E}-03$ \\
\hline LOC100496628-like.S & 6.05 & $4.31 \mathrm{E}-15$ \\
\hline sostdc1.S & 6.04 & $2.05 \mathrm{E}-02$ \\
\hline adcyap1.L & 6.04 & $5.59 \mathrm{E}-05$ \\
\hline Xelaev18044103m.g & 6.01 & $4.81 \mathrm{E}-04$ \\
\hline six1.S & 6.01 & $4.12 \mathrm{E}-03$ \\
\hline zic3.S & 5.99 & $1.06 \mathrm{E}-03$ \\
\hline mecom.L & 5.99 & $4.08 \mathrm{E}-06$ \\
\hline wnt8a.L & 5.99 & $7.45 \mathrm{E}-04$ \\
\hline slc3a2.L & 5.96 & $7.20 \mathrm{E}-03$ \\
\hline plod2.S & 5.96 & $4.04 \mathrm{E}-02$ \\
\hline sp5.L & 5.95 & $9.89 \mathrm{E}-03$ \\
\hline $\operatorname{limd2.L}$ & 5.95 & $7.84 \mathrm{E}-23$ \\
\hline bmp3.L & 5.95 & $2.87 \mathrm{E}-02$ \\
\hline pck1.L & 5.93 & $3.80 \mathrm{E}-14$ \\
\hline tfap2b.L & 5.92 & $8.73 E-21$ \\
\hline Scrt1.L & 5.92 & $1.75 \mathrm{E}-05$ \\
\hline tpbg.L & 5.91 & $3.02 \mathrm{E}-03$ \\
\hline dkk1.L & 5.91 & $3.92 \mathrm{E}-02$ \\
\hline gria1-like.1.S & 5.91 & $1.48 \mathrm{E}-12$ \\
\hline slc1a3.L & 5.91 & $1.60 \mathrm{E}-02$ \\
\hline myct1.L & 5.90 & $1.04 \mathrm{E}-03$ \\
\hline Xetrov90000859m.L & 5.89 & $1.72 \mathrm{E}-10$ \\
\hline
\end{tabular}




\begin{tabular}{|c|c|c|}
\hline cldn3.L & 5.89 & $5.43 \mathrm{E}-03$ \\
\hline skor1.L & 5.83 & $1.02 \mathrm{E}-05$ \\
\hline kif26a.S & 5.83 & $1.39 \mathrm{E}-17$ \\
\hline gad1.1.S & 5.83 & $1.72 \mathrm{E}-05$ \\
\hline hcrtr2.L & 5.79 & $1.66 \mathrm{E}-02$ \\
\hline LOC100495096.1 & 5.77 & $1.43 \mathrm{E}-02$ \\
\hline tmem35.L & 5.75 & $1.33 \mathrm{E}-12$ \\
\hline Xelaev18024653m.g & 5.75 & $1.40 \mathrm{E}-06$ \\
\hline Xelaev18025499m.g & 5.74 & $3.60 \mathrm{E}-05$ \\
\hline cyp1a1.S & 5.73 & $3.42 \mathrm{E}-02$ \\
\hline neurog2.S & 5.72 & 1.55E-03 \\
\hline kcnj2.S & 5.70 & $1.08 \mathrm{E}-02$ \\
\hline bhlhe22.L & 5.68 & $3.35 \mathrm{E}-02$ \\
\hline$n k \times 6-2 . L$ & 5.67 & $7.65 \mathrm{E}-04$ \\
\hline neurog2.L & 5.67 & $3.40 \mathrm{E}-02$ \\
\hline sall3.L & 5.66 & $2.74 \mathrm{E}-10$ \\
\hline snap25.L & 5.63 & $3.22 \mathrm{E}-24$ \\
\hline $\operatorname{drgx} . L$ & 5.62 & $2.70 \mathrm{E}-04$ \\
\hline$c p / x 2 . L$ & 5.57 & $1.26 \mathrm{E}-15$ \\
\hline mamdc2.L & 5.55 & $6.26 \mathrm{E}-05$ \\
\hline tubb2b.S & 5.55 & $1.31 \mathrm{E}-07$ \\
\hline kiaa0408.L & 5.55 & 4.64E-02 \\
\hline tox2.S & 5.55 & $6.62 \mathrm{E}-25$ \\
\hline stxbp1.S & 5.53 & $6.87 \mathrm{E}-03$ \\
\hline pax8.L & 5.52 & $2.82 \mathrm{E}-09$ \\
\hline LOC100487796-like.L & 5.50 & $1.23 \mathrm{E}-25$ \\
\hline Xelaev18026630m.g & 5.49 & 5.46E-06 \\
\hline pla2g12b.S & 5.48 & $3.04 \mathrm{E}-02$ \\
\hline st18.S & 5.47 & $1.39 \mathrm{E}-06$ \\
\hline kank2.L & 5.47 & $4.16 \mathrm{E}-02$ \\
\hline homer3.L & 5.46 & $2.63 \mathrm{E}-02$ \\
\hline Xelaev18015119m.g & 5.44 & $1.65 \mathrm{E}-06$ \\
\hline c8orf46.S & 5.41 & $5.50 \mathrm{E}-05$ \\
\hline Xelaev18039076m.g & 5.41 & $5.02 \mathrm{E}-26$ \\
\hline Xetrov90016928m.L & 5.39 & $5.16 \mathrm{E}-04$ \\
\hline Xelaev18007001m.g & 5.39 & $2.00 \mathrm{E}-14$ \\
\hline hapln3.S & 5.37 & $3.19 \mathrm{E}-08$ \\
\hline c8orf46.L & 5.37 & 7.03E-03 \\
\hline
\end{tabular}




\begin{tabular}{|c|c|c|}
\hline igf2.L & 5.36 & $2.49 \mathrm{E}-03$ \\
\hline st8sia1.S & 5.36 & $1.61 \mathrm{E}-02$ \\
\hline cbfa2t3.L & 5.36 & $2.32 \mathrm{E}-06$ \\
\hline pax8.S & 5.35 & $7.94 \mathrm{E}-03$ \\
\hline $\operatorname{six1.L}$ & 5.35 & $2.54 \mathrm{E}-02$ \\
\hline rph3al.S & 5.33 & 1.10E-03 \\
\hline Xelaev18024617m.g & 5.33 & $7.28 \mathrm{E}-12$ \\
\hline Xelaev18047557m.g & 5.32 & $2.01 \mathrm{E}-02$ \\
\hline gpx3.S & 5.31 & $2.55 \mathrm{E}-03$ \\
\hline slc43a2.S & 5.28 & $1.82 \mathrm{E}-28$ \\
\hline tfap2e.S & 5.24 & $6.45 \mathrm{E}-14$ \\
\hline Xetrov90008930m.S & 5.24 & $1.45 \mathrm{E}-03$ \\
\hline Sox9.L & 5.24 & $2.40 \mathrm{E}-02$ \\
\hline adora2a.L & 5.24 & $7.28 \mathrm{E}-03$ \\
\hline aldh1a2.L & 5.24 & 2.27E-09 \\
\hline LOC100496446.L & 5.22 & 1.57E-02 \\
\hline celf2.L & 5.22 & $4.54 \mathrm{E}-13$ \\
\hline hoxc3.S & 5.21 & 1.16E-02 \\
\hline pmp22.L & 5.21 & $1.74 \mathrm{E}-12$ \\
\hline Xelaev18021728m.g & 5.17 & $1.53 \mathrm{E}-12$ \\
\hline mcf2I.S & 5.16 & $2.81 \mathrm{E}-09$ \\
\hline gpr63.S & 5.15 & $2.37 \mathrm{E}-07$ \\
\hline kremen2.S & 5.10 & 9.50E-07 \\
\hline hes5_X2.S & 5.09 & $4.52 \mathrm{E}-04$ \\
\hline Xelaev18015305m.g & 5.09 & $1.40 \mathrm{E}-02$ \\
\hline$d b n 1 . L$ & 5.08 & $3.22 \mathrm{E}-27$ \\
\hline Xelaev18018196m.g & 5.08 & $1.63 \mathrm{E}-06$ \\
\hline$d 1 \times 6 . L$ & 5.07 & $1.68 \mathrm{E}-19$ \\
\hline Xelaev18016582m.g & 5.07 & $5.34 \mathrm{E}-19$ \\
\hline sacs.L & 5.07 & 1.16E-05 \\
\hline$d b n 1 . S$ & 5.06 & $2.56 \mathrm{E}-20$ \\
\hline dnajc6.S & 5.05 & $1.83 \mathrm{E}-08$ \\
\hline nrp1.L & 5.05 & $5.16 \mathrm{E}-12$ \\
\hline nrp2.L & 5.04 & $1.64 \mathrm{E}-16$ \\
\hline$a b c d 2 . S$ & 5.04 & $4.17 \mathrm{E}-02$ \\
\hline Xelaev18005831m.g & 5.04 & $3.34 \mathrm{E}-05$ \\
\hline Xetrov90016831m.L & 5.03 & 5.99E-05 \\
\hline jam3.S & 5.03 & $1.25 \mathrm{E}-05$ \\
\hline
\end{tabular}




\begin{tabular}{|c|c|c|}
\hline tmeff1.S & 5.02 & 1.14E-09 \\
\hline insm1.L & 4.99 & 8.73E-03 \\
\hline gli2.S & 4.98 & $6.18 \mathrm{E}-11$ \\
\hline kif26a.L & 4.95 & 5.42E-09 \\
\hline fam198b.L & 4.94 & 8.39E-03 \\
\hline runx1t1.S & 4.94 & $9.66 \mathrm{E}-09$ \\
\hline crabp2.S & 4.90 & $1.25 \mathrm{E}-10$ \\
\hline syt2-like.S & 4.90 & $1.96 \mathrm{E}-03$ \\
\hline clip2.S & 4.89 & 1.16E-04 \\
\hline npr3.L & 4.88 & $1.55 \mathrm{E}-02$ \\
\hline LOC101734468.L & 4.87 & $4.56 \mathrm{E}-02$ \\
\hline arhgap4.L & 4.87 & $1.42 \mathrm{E}-18$ \\
\hline rab3b.S & 4.87 & $4.16 \mathrm{E}-04$ \\
\hline pax2.S & 4.86 & 1.83E-09 \\
\hline$t b \times 22 . S$ & 4.85 & $4.31 \mathrm{E}-02$ \\
\hline tuba4a.L & 4.85 & $3.70 \mathrm{E}-23$ \\
\hline slc38a8.L & 4.83 & $5.61 \mathrm{E}-03$ \\
\hline ube2q/1.S & 4.82 & $2.40 \mathrm{E}-02$ \\
\hline $\operatorname{arx} . L$ & 4.80 & $4.96 \mathrm{E}-02$ \\
\hline$a s b 2 . L$ & 4.80 & $2.07 \mathrm{E}-03$ \\
\hline$d m b \times 1 . S$ & 4.78 & $1.40 \mathrm{E}-04$ \\
\hline flnc.S & 4.78 & 1.77E-08 \\
\hline plekhg4.S & 4.77 & $6.24 \mathrm{E}-07$ \\
\hline Xelaev18044320m.g & 4.77 & $3.66 \mathrm{E}-05$ \\
\hline$n r 2 f 2 . L$ & 4.73 & $1.76 \mathrm{E}-03$ \\
\hline hdac7.S & 4.73 & $2.27 \mathrm{E}-05$ \\
\hline cacnb3.L & 4.72 & 3.97E-02 \\
\hline LOC100488523.L & 4.72 & $1.99 \mathrm{E}-03$ \\
\hline$n k \times 2-6 . S$ & 4.70 & $1.97 \mathrm{E}-04$ \\
\hline pak3.S & 4.70 & $5.13 \mathrm{E}-21$ \\
\hline Xelaev18043580m.g & 4.69 & $3.76 \mathrm{E}-24$ \\
\hline Sox11.L & 4.66 & $3.76 \mathrm{E}-04$ \\
\hline hes5.3.S & 4.66 & $5.74 \mathrm{E}-04$ \\
\hline fstl1.S & 4.66 & $6.57 \mathrm{E}-08$ \\
\hline Xelaev18013307m.g & 4.64 & $4.51 \mathrm{E}-02$ \\
\hline Xelaev18042154m.g & 4.64 & $1.28 \mathrm{E}-08$ \\
\hline kif5a.L & 4.63 & $6.32 \mathrm{E}-03$ \\
\hline faxc.L & 4.63 & $3.01 \mathrm{E}-12$ \\
\hline
\end{tabular}




\begin{tabular}{|c|c|c|}
\hline bcam.S & 4.62 & 9.73E-06 \\
\hline emilin1.L & 4.62 & $5.88 \mathrm{E}-13$ \\
\hline zic3.L & 4.61 & 3.97E-04 \\
\hline six3.S & 4.60 & $5.50 \mathrm{E}-09$ \\
\hline bmpr1b.L & 4.58 & $2.91 \mathrm{E}-02$ \\
\hline st6galnac6.L & 4.57 & 3.88E-03 \\
\hline Xelaev18025917m.g & 4.56 & $3.15 \mathrm{E}-06$ \\
\hline adamts/1.L & 4.55 & $1.80 \mathrm{E}-11$ \\
\hline $\operatorname{scg} 3 . L$ & 4.55 & $1.71 \mathrm{E}-02$ \\
\hline lef1.S & 4.55 & 1.18E-05 \\
\hline LOC100489483-like.S & 4.55 & 1.64E-02 \\
\hline pak3.L & 4.55 & 7.82E-05 \\
\hline rgs9bp.S & 4.53 & 6.82E-05 \\
\hline runx1t1.L & 4.53 & $1.12 \mathrm{E}-05$ \\
\hline aldh1a2.S & 4.53 & $8.36 \mathrm{E}-17$ \\
\hline slco5a1.S & 4.52 & $4.40 \mathrm{E}-07$ \\
\hline limd2.S & 4.51 & 5.67E-05 \\
\hline b4galnt1.S & 4.49 & $1.10 \mathrm{E}-08$ \\
\hline gfra1.S & 4.46 & $2.12 \mathrm{E}-11$ \\
\hline plekho1.L & 4.46 & $4.33 \mathrm{E}-05$ \\
\hline nckap5l.L & 4.45 & $1.46 \mathrm{E}-19$ \\
\hline prph.L & 4.45 & $4.92 \mathrm{E}-14$ \\
\hline hdac9.S & 4.44 & $4.00 \mathrm{E}-02$ \\
\hline cited2.L & 4.43 & $7.42 \mathrm{E}-14$ \\
\hline ebf3.L & 4.42 & 3.97E-03 \\
\hline pitx2.L & 4.39 & $4.02 \mathrm{E}-03$ \\
\hline otx2.L & 4.38 & 2.27E-09 \\
\hline unc5a.L & 4.37 & $4.99 \mathrm{E}-03$ \\
\hline ssbp4.S & 4.37 & $4.02 \mathrm{E}-03$ \\
\hline Xelaev18019210m.g & 4.37 & $2.19 \mathrm{E}-13$ \\
\hline unc45b.S & 4.36 & $2.60 \mathrm{E}-12$ \\
\hline tmem116.S & 4.36 & $6.02 \mathrm{E}-09$ \\
\hline cpe.S & 4.35 & $2.85 \mathrm{E}-07$ \\
\hline auts2.S & 4.34 & $4.42 \mathrm{E}-07$ \\
\hline CXCr2.L & 4.34 & $1.08 \mathrm{E}-02$ \\
\hline insm2.L & 4.33 & $2.09 \mathrm{E}-06$ \\
\hline fgf16.L & 4.32 & $3.10 \mathrm{E}-07$ \\
\hline des.1.S & 4.31 & $9.24 \mathrm{E}-06$ \\
\hline
\end{tabular}




\begin{tabular}{|c|c|c|}
\hline six3.L & 4.30 & 1.56E-29 \\
\hline srrm4.L & 4.29 & $6.76 \mathrm{E}-05$ \\
\hline tcf15.L & 4.29 & 5.39E-04 \\
\hline pip4k2b.L & 4.25 & $1.77 \mathrm{E}-16$ \\
\hline pou3f2.S & 4.25 & $9.24 \mathrm{E}-05$ \\
\hline cacna2d1.L & 4.25 & $7.42 \mathrm{E}-04$ \\
\hline bag2.L & 4.23 & $7.74 \mathrm{E}-04$ \\
\hline cacna2d2.L & 4.21 & $1.41 \mathrm{E}-04$ \\
\hline itga8.L & 4.21 & $2.60 \mathrm{E}-03$ \\
\hline sfrp2.L & 4.20 & $1.67 \mathrm{E}-20$ \\
\hline skor2.L & 4.19 & $7.95 \mathrm{E}-03$ \\
\hline itgb8.L & 4.18 & $1.98 \mathrm{E}-02$ \\
\hline ppp1r9a.S & 4.17 & $2.84 \mathrm{E}-02$ \\
\hline$n k x 6-1 . L$ & 4.16 & 9.80E-04 \\
\hline Xelaev18024654m.g & 4.16 & $7.21 \mathrm{E}-08$ \\
\hline cep85I.L & 4.15 & $6.18 \mathrm{E}-08$ \\
\hline Irat.S & 4.15 & $5.56 \mathrm{E}-03$ \\
\hline pax6.S & 4.14 & $5.29 \mathrm{E}-09$ \\
\hline zfhx4.S & 4.12 & $1.85 \mathrm{E}-14$ \\
\hline pmp22.S & 4.11 & $4.06 \mathrm{E}-13$ \\
\hline zcchc24.L & 4.10 & $9.16 \mathrm{E}-03$ \\
\hline cbfa2t2.L & 4.10 & $9.62 \mathrm{E}-12$ \\
\hline eln2.L & 4.09 & $9.78 \mathrm{E}-03$ \\
\hline$t / x 3 . L$ & 4.09 & $1.74 \mathrm{E}-02$ \\
\hline evi5.L & 4.08 & $4.52 \mathrm{E}-26$ \\
\hline rgmb.L & 4.08 & 7.61E-05 \\
\hline $\operatorname{lh} \times 5 . L$ & 4.08 & 2.25E-02 \\
\hline cyp26c1.L & 4.07 & 9.77E-04 \\
\hline dnajc6.L & 4.05 & $1.12 \mathrm{E}-07$ \\
\hline hes2.L & 4.04 & $1.27 \mathrm{E}-04$ \\
\hline dpys/3.S & 4.03 & $1.68 \mathrm{E}-19$ \\
\hline klf7.L & 4.02 & $1.40 \mathrm{E}-02$ \\
\hline pde11a.S & 4.02 & $1.22 \mathrm{E}-02$ \\
\hline pde11a.S & 4.02 & $1.22 \mathrm{E}-02$ \\
\hline crmp1.L & 4.01 & $1.00 \mathrm{E}-02$ \\
\hline mctp2.L & 4.01 & $4.11 \mathrm{E}-05$ \\
\hline $\operatorname{vim} . S$ & 4.00 & 5.77E-03 \\
\hline fam212a.L & 3.99 & $4.79 \mathrm{E}-08$ \\
\hline
\end{tabular}




\begin{tabular}{|c|c|c|}
\hline Xelaev18004886m.g & 3.99 & $2.16 \mathrm{E}-08$ \\
\hline nuak1.L & 3.98 & 3.50E-07 \\
\hline sncb.L & 3.98 & $8.27 \mathrm{E}-10$ \\
\hline tdrp.L & 3.98 & $3.22 E-24$ \\
\hline pcdh8.L & 3.98 & $1.45 \mathrm{E}-03$ \\
\hline serpinf1.L & 3.97 & 1.35E-04 \\
\hline ror2.L & 3.94 & $1.25 \mathrm{E}-10$ \\
\hline map3k13.S & 3.94 & 2.90E-02 \\
\hline Xelaev18008189m.g & 3.93 & $4.58 \mathrm{E}-05$ \\
\hline cbfa2t2.S & 3.92 & $7.07 \mathrm{E}-13$ \\
\hline$m e x 3 b . L$ & 3.92 & $1.10 \mathrm{E}-09$ \\
\hline gngt2.1.S & 3.92 & $3.68 \mathrm{E}-02$ \\
\hline cdc42ep3.S & 3.90 & $3.95 \mathrm{E}-07$ \\
\hline arl8a.S & 3.89 & $1.72 \mathrm{E}-04$ \\
\hline Xelaev18003346m.g & 3.89 & $3.48 \mathrm{E}-03$ \\
\hline prr5l.S & 3.88 & $6.16 \mathrm{E}-03$ \\
\hline scn8a.L & 3.88 & $2.89 \mathrm{E}-02$ \\
\hline elav/3.L & 3.88 & $5.43 \mathrm{E}-18$ \\
\hline fam110b.L & 3.87 & $1.11 \mathrm{E}-06$ \\
\hline foxg1.L & 3.86 & $7.65 \mathrm{E}-08$ \\
\hline Xelaev18023457m.g & 3.86 & $1.01 \mathrm{E}-03$ \\
\hline mef2d.S & 3.86 & $1.73 \mathrm{E}-08$ \\
\hline zc3h12c.L & 3.85 & $8.45 \mathrm{E}-15$ \\
\hline slc6a5.L & 3.84 & $4.72 \mathrm{E}-02$ \\
\hline LOC100494817.L & 3.82 & $9.87 \mathrm{E}-06$ \\
\hline dpys/4.S & 3.82 & $4.34 \mathrm{E}-02$ \\
\hline hes8.L & 3.82 & $1.74 \mathrm{E}-12$ \\
\hline dbndd1.L & 3.80 & $2.05 \mathrm{E}-05$ \\
\hline $\operatorname{lh} \times 5 . S$ & 3.78 & $1.52 \mathrm{E}-06$ \\
\hline dennd2c.L & 3.78 & $9.03 \mathrm{E}-11$ \\
\hline hes10.L & 3.77 & $1.18 \mathrm{E}-06$ \\
\hline gcnt1.S & 3.75 & $1.18 \mathrm{E}-05$ \\
\hline hes5.1.L & 3.74 & $4.31 \mathrm{E}-06$ \\
\hline$m d k \cdot L$ & 3.73 & $3.40 \mathrm{E}-17$ \\
\hline tox.L & 3.73 & $1.04 \mathrm{E}-05$ \\
\hline spon1.S & 3.72 & $5.18 \mathrm{E}-04$ \\
\hline cdc42bpa.S & 3.71 & $1.04 \mathrm{E}-13$ \\
\hline adamdec1.L & 3.71 & 1.10E-02 \\
\hline
\end{tabular}




\begin{tabular}{|c|c|c|}
\hline fn1.S & 3.70 & $1.47 \mathrm{E}-19$ \\
\hline Xelaev18037602m.g & 3.69 & $7.76 \mathrm{E}-10$ \\
\hline map2.L & 3.69 & $5.12 \mathrm{E}-05$ \\
\hline sox9.S & 3.68 & 1.89E-05 \\
\hline edn1.L & 3.67 & $2.50 \mathrm{E}-03$ \\
\hline hnrnpll.S & 3.67 & $2.69 \mathrm{E}-13$ \\
\hline$m t c / 1 . L$ & 3.67 & $1.81 \mathrm{E}-04$ \\
\hline mycl.S & 3.67 & $3.02 \mathrm{E}-08$ \\
\hline nr5a2.S & 3.66 & $1.22 \mathrm{E}-08$ \\
\hline nox4.S & 3.64 & $6.16 \mathrm{E}-03$ \\
\hline dII1.L & 3.62 & $9.68 \mathrm{E}-07$ \\
\hline Xelaev18044758m.g & 3.61 & 4.33E-02 \\
\hline sgip1.L & 3.60 & $1.23 \mathrm{E}-06$ \\
\hline Xelaev18020376m.g & 3.60 & $1.01 \mathrm{E}-02$ \\
\hline klh/35.S & 3.60 & $1.38 \mathrm{E}-03$ \\
\hline st3gal2.2.L & 3.60 & $3.57 \mathrm{E}-02$ \\
\hline sox11.S & 3.58 & $1.43 \mathrm{E}-02$ \\
\hline nceh1.L & 3.57 & $2.09 \mathrm{E}-05$ \\
\hline manea.S & 3.57 & $3.67 \mathrm{E}-03$ \\
\hline ulk1.L & 3.56 & $2.07 \mathrm{E}-08$ \\
\hline spon1.L & 3.56 & 9.47E-06 \\
\hline rnf165.L & 3.56 & $1.22 \mathrm{E}-03$ \\
\hline$c p / x 2 . S$ & 3.55 & $2.37 \mathrm{E}-02$ \\
\hline dst.L & 3.54 & 8.87E-05 \\
\hline slc43a2.L & 3.54 & $2.77 \mathrm{E}-07$ \\
\hline$m x i 1 . S$ & 3.54 & $5.02 \mathrm{E}-05$ \\
\hline gsta1.L & 3.53 & $5.27 \mathrm{E}-03$ \\
\hline cdc42ep4.L & 3.53 & $3.79 \mathrm{E}-04$ \\
\hline kcnq3.S & 3.52 & 2.77E-07 \\
\hline ncam1.S & 3.52 & $6.76 \mathrm{E}-11$ \\
\hline creb3/1.S & 3.51 & $1.03 \mathrm{E}-07$ \\
\hline aplnr.S & 3.51 & $4.93 \mathrm{E}-04$ \\
\hline$x k r 4 . L$ & 3.49 & 1.39E-06 \\
\hline Irch2.L & 3.48 & $9.72 \mathrm{E}-08$ \\
\hline sp5I.S & 3.48 & $2.17 \mathrm{E}-03$ \\
\hline gfi1.L & 3.47 & $1.85 \mathrm{E}-02$ \\
\hline dennd2c.S & 3.46 & $1.83 \mathrm{E}-07$ \\
\hline zic2.L & 3.44 & $2.27 \mathrm{E}-04$ \\
\hline
\end{tabular}




\begin{tabular}{|c|c|c|}
\hline rassf6.L & 3.44 & $2.51 \mathrm{E}-03$ \\
\hline hes9-1.L & 3.44 & $6.62 \mathrm{E}-04$ \\
\hline dact1.L & 3.43 & $2.38 \mathrm{E}-06$ \\
\hline st8sia1.L & 3.43 & $3.02 \mathrm{E}-05$ \\
\hline wipf1.L & 3.42 & $1.64 \mathrm{E}-02$ \\
\hline eya1.S & 3.42 & $4.20 \mathrm{E}-07$ \\
\hline slco5a1.L & 3.41 & 3.63E-09 \\
\hline$f b \times 010 . L$ & 3.41 & $1.35 \mathrm{E}-10$ \\
\hline sox4.S & 3.41 & $5.45 \mathrm{E}-05$ \\
\hline LOC105946937.L & 3.41 & $2.26 \mathrm{E}-05$ \\
\hline dusp4.S & 3.40 & $9.50 \mathrm{E}-07$ \\
\hline$z b t b 16 . S$ & 3.40 & $7.24 \mathrm{E}-05$ \\
\hline LOC100490436.L & 3.39 & $2.57 \mathrm{E}-02$ \\
\hline cdc42se2-like.1.L & 3.39 & $1.64 \mathrm{E}-09$ \\
\hline LOC100494502.L & 3.38 & $1.14 \mathrm{E}-02$ \\
\hline$a p 3 b 2 . L$ & 3.38 & $2.70 \mathrm{E}-04$ \\
\hline bmpr1b.S & 3.37 & $1.95 \mathrm{E}-03$ \\
\hline$a s b 1 . L$ & 3.37 & $2.15 \mathrm{E}-07$ \\
\hline firt3.L & 3.37 & $7.45 \mathrm{E}-04$ \\
\hline fam134b.S & 3.37 & $1.29 \mathrm{E}-02$ \\
\hline dlc.S & 3.35 & 8.87E-05 \\
\hline crabp2.L & 3.34 & $1.08 \mathrm{E}-05$ \\
\hline Imcd1.S & 3.34 & $2.63 \mathrm{E}-02$ \\
\hline gch1.L & 3.34 & $6.12 \mathrm{E}-05$ \\
\hline gli2.L & 3.34 & $4.31 \mathrm{E}-11$ \\
\hline el/3.L & 3.32 & $4.08 \mathrm{E}-02$ \\
\hline ag1.S & 3.32 & $1.31 \mathrm{E}-10$ \\
\hline tmod4.S & 3.32 & $2.31 \mathrm{E}-02$ \\
\hline Xelaev18030284m.g & 3.31 & $4.39 \mathrm{E}-03$ \\
\hline hes9-1.S & 3.31 & $7.49 \mathrm{E}-14$ \\
\hline sesn1.L & 3.31 & $4.16 \mathrm{E}-06$ \\
\hline sulf1.S & 3.31 & $3.55 \mathrm{E}-03$ \\
\hline$m c f 2 I .2 . L$ & 3.30 & $2.56 \mathrm{E}-05$ \\
\hline rundc3a.L & 3.30 & $2.58 \mathrm{E}-04$ \\
\hline LOC101732576.S & 3.30 & $4.01 \mathrm{E}-02$ \\
\hline ppp1r14b.S & 3.29 & $3.10 \mathrm{E}-07$ \\
\hline tmem169.S & 3.29 & $3.01 \mathrm{E}-04$ \\
\hline arid1b.L & 3.28 & $1.99 \mathrm{E}-13$ \\
\hline
\end{tabular}




\begin{tabular}{|c|c|c|}
\hline reep5.S & 3.28 & $2.29 \mathrm{E}-24$ \\
\hline Cd82.L & 3.26 & 4.77E-07 \\
\hline fam43a.S & 3.25 & $1.79 \mathrm{E}-03$ \\
\hline cttnbp2nl.L & 3.25 & $4.11 \mathrm{E}-04$ \\
\hline arhgef26.S & 3.25 & $1.08 \mathrm{E}-02$ \\
\hline sfrp2.S & 3.24 & $3.87 E-39$ \\
\hline sparc.L & 3.24 & $7.18 \mathrm{E}-04$ \\
\hline tmem169.L & 3.22 & $4.37 \mathrm{E}-04$ \\
\hline fyn-like.L & 3.22 & $6.87 \mathrm{E}-03$ \\
\hline Xelaev18027950m.g & 3.21 & $4.03 \mathrm{E}-10$ \\
\hline plekhg1.L & 3.21 & $6.37 \mathrm{E}-06$ \\
\hline Xelaev18044942m.g & 3.19 & $3.18 \mathrm{E}-02$ \\
\hline ak3.S & 3.19 & $3.89 \mathrm{E}-10$ \\
\hline Irpprc.S & 3.19 & $5.15 \mathrm{E}-04$ \\
\hline dclk1.S & 3.19 & $9.03 \mathrm{E}-03$ \\
\hline tmem158.L & 3.19 & $1.36 \mathrm{E}-05$ \\
\hline Irp4.L & 3.17 & $1.12 \mathrm{E}-05$ \\
\hline irx1.L & 3.17 & $1.23 \mathrm{E}-06$ \\
\hline st6galnac1.S & 3.17 & $2.50 \mathrm{E}-03$ \\
\hline bcar1.S & 3.15 & $1.41 \mathrm{E}-04$ \\
\hline LOC100498409-like.S & 3.15 & $2.72 \mathrm{E}-03$ \\
\hline LOC100170576.L & 3.15 & $7.02 \mathrm{E}-10$ \\
\hline otx1.S & 3.14 & 9.33E-06 \\
\hline MGC147600.S & 3.13 & $3.85 \mathrm{E}-02$ \\
\hline eya2.S & 3.12 & $6.03 \mathrm{E}-04$ \\
\hline fmnl2-like.S & 3.12 & $3.61 \mathrm{E}-02$ \\
\hline pkdcc.L & 3.11 & $2.80 \mathrm{E}-15$ \\
\hline mybl1.S & 3.11 & $2.22 \mathrm{E}-02$ \\
\hline nr2e1.S & 3.11 & $4.31 \mathrm{E}-03$ \\
\hline Xelaev18024436m.g & 3.11 & $1.94 \mathrm{E}-06$ \\
\hline fgfr1.S & 3.10 & $8.39 \mathrm{E}-13$ \\
\hline LOC100487080-like.L & 3.10 & $1.46 \mathrm{E}-02$ \\
\hline cdk5r1.S & 3.10 & $5.85 \mathrm{E}-06$ \\
\hline arhgef40.S & 3.10 & $1.34 \mathrm{E}-02$ \\
\hline LOC101730599.S & 3.10 & $1.29 \mathrm{E}-03$ \\
\hline egln2.L & 3.09 & $9.62 \mathrm{E}-04$ \\
\hline$e m \times 2 . S$ & 3.09 & $4.41 \mathrm{E}-02$ \\
\hline pag1.S & 3.08 & $2.06 \mathrm{E}-05$ \\
\hline
\end{tabular}




\begin{tabular}{|c|c|c|}
\hline psat1.L & 3.07 & 2.34E-05 \\
\hline sulf1.L & 3.07 & $2.74 \mathrm{E}-10$ \\
\hline cel-like.1.S & 3.06 & $2.21 \mathrm{E}-02$ \\
\hline zfhx4.L & 3.06 & 2.63E-09 \\
\hline Xelaev18029662m.g & 3.05 & 2.77E-02 \\
\hline znf219.L & 3.04 & 3.06E-03 \\
\hline plekhg4.L & 3.04 & $1.80 \mathrm{E}-07$ \\
\hline$a b c b 9 . L$ & 3.03 & $1.48 \mathrm{E}-05$ \\
\hline tnni1.2.L & 3.02 & $4.82 \mathrm{E}-02$ \\
\hline tuba4a.S & 3.02 & $1.22 \mathrm{E}-03$ \\
\hline pdia2.S & 3.01 & 2.87E-02 \\
\hline rnf165.S & 3.00 & $4.61 \mathrm{E}-12$ \\
\hline trabd2a.L & 3.00 & $7.06 \mathrm{E}-07$ \\
\hline ptch2.S & 2.99 & $2.26 \mathrm{E}-02$ \\
\hline ngfr.L & 2.98 & $1.43 E-06$ \\
\hline manea.L & 2.98 & $1.62 \mathrm{E}-03$ \\
\hline ptprt.L & 2.97 & $4.02 \mathrm{E}-03$ \\
\hline tpbg.S & 2.97 & 1.17E-04 \\
\hline pik3r3.L & 2.97 & $1.18 \mathrm{E}-04$ \\
\hline afap1.S & 2.96 & $3.94 \mathrm{E}-03$ \\
\hline$c b / b . L$ & 2.94 & $3.52 \mathrm{E}-06$ \\
\hline plxnb1.L & 2.94 & $5.45 \mathrm{E}-09$ \\
\hline ptch1.L & 2.93 & $8.95 \mathrm{E}-06$ \\
\hline Xelaev18016487m.g & 2.93 & 2.37E-02 \\
\hline fdft1.L & 2.93 & $1.02 \mathrm{E}-03$ \\
\hline fgfr4.L & 2.93 & $1.34 \mathrm{E}-11$ \\
\hline prickle1.S & 2.92 & 7.63E-09 \\
\hline spsb4-like.L & 2.92 & $1.33 \mathrm{E}-03$ \\
\hline pitx2.S & 2.92 & $2.76 \mathrm{E}-02$ \\
\hline dclk1.L & 2.91 & $2.70 \mathrm{E}-02$ \\
\hline rgma.L & 2.91 & $1.09 \mathrm{E}-06$ \\
\hline LOC100498368-like.L & 2.90 & $2.04 \mathrm{E}-02$ \\
\hline smim3.S & 2.90 & $7.08 \mathrm{E}-04$ \\
\hline amot/2.L & 2.90 & $1.15 \mathrm{E}-08$ \\
\hline gas7.L & 2.89 & $5.18 \mathrm{E}-03$ \\
\hline mgc69520.S & 2.88 & $2.15 \mathrm{E}-07$ \\
\hline slit1.S & 2.88 & $2.77 \mathrm{E}-02$ \\
\hline sox4.L & 2.88 & $8.44 \mathrm{E}-04$ \\
\hline
\end{tabular}




\begin{tabular}{|c|c|c|}
\hline myh6.L & 2.88 & $1.72 \mathrm{E}-02$ \\
\hline ncam1.L & 2.88 & $9.12 \mathrm{E}-06$ \\
\hline Xelaev18044438m.g & 2.87 & $9.51 \mathrm{E}-03$ \\
\hline znf608.S & 2.87 & $6.99 \mathrm{E}-16$ \\
\hline Xelaev18003796m.g & 2.86 & 1.67E-02 \\
\hline homer1.S & 2.85 & $7.12 \mathrm{E}-03$ \\
\hline tmem132a.L & 2.85 & $5.71 \mathrm{E}-04$ \\
\hline il17rd.S & 2.85 & 2.47E-04 \\
\hline Xetrov90002103m.L & 2.85 & $3.27 \mathrm{E}-03$ \\
\hline $\operatorname{Im} x 1 b .1 . L$ & 2.85 & $6.18 \mathrm{E}-03$ \\
\hline ankrd65.L & 2.85 & $2.70 \mathrm{E}-03$ \\
\hline akap12.L & 2.83 & $1.65 \mathrm{E}-06$ \\
\hline rfk.S & 2.82 & $8.23 \mathrm{E}-04$ \\
\hline Ifng.L & 2.82 & $4.22 \mathrm{E}-10$ \\
\hline$m t c / 1 . S$ & 2.82 & 1.19E-02 \\
\hline mex3a.S & 2.81 & $7.45 \mathrm{E}-04$ \\
\hline Xelaev18015889m.g & 2.81 & $4.70 \mathrm{E}-03$ \\
\hline hes3.3.L & 2.80 & $1.04 \mathrm{E}-06$ \\
\hline calb1.S & 2.80 & $2.20 \mathrm{E}-02$ \\
\hline kank3.L & 2.80 & $1.07 \mathrm{E}-03$ \\
\hline Xetrov90009914m.S & 2.79 & $1.24 \mathrm{E}-04$ \\
\hline arhgap36.L & 2.79 & $1.70 \mathrm{E}-11$ \\
\hline scamp4.S & 2.79 & 4.96E-04 \\
\hline gmnn.L & 2.79 & $4.12 \mathrm{E}-20$ \\
\hline rai2.S & 2.79 & $2.04 \mathrm{E}-03$ \\
\hline st3gal6.L & 2.78 & $1.42 \mathrm{E}-04$ \\
\hline mamld1.L & 2.77 & $2.18 \mathrm{E}-02$ \\
\hline afap1/1.L & 2.77 & $2.27 \mathrm{E}-19$ \\
\hline dsc3.S & 2.77 & $8.95 \mathrm{E}-03$ \\
\hline ndst1.S & 2.77 & $2.95 \mathrm{E}-07$ \\
\hline Xelaev18010427m.g & 2.76 & $2.53 \mathrm{E}-04$ \\
\hline rbm38.S & 2.76 & $6.82 \mathrm{E}-10$ \\
\hline LOC100492579.S & 2.76 & $2.49 \mathrm{E}-03$ \\
\hline rnf130.S & 2.75 & $4.50 \mathrm{E}-02$ \\
\hline Irrn1-like.1.L & 2.75 & $8.62 \mathrm{E}-08$ \\
\hline plxnb1.S & 2.75 & $1.71 \mathrm{E}-04$ \\
\hline spry2.L & 2.74 & $7.60 \mathrm{E}-03$ \\
\hline vcan.L & 2.74 & 4.26E-04 \\
\hline
\end{tabular}




\begin{tabular}{|c|c|c|}
\hline auts2.L & 2.73 & $8.62 \mathrm{E}-08$ \\
\hline ulk1.S & 2.73 & 7.06E-06 \\
\hline pros1.S & 2.73 & 2.05E-04 \\
\hline prtg.L & 2.73 & $1.55 \mathrm{E}-02$ \\
\hline crmp1.S & 2.73 & $1.27 \mathrm{E}-02$ \\
\hline Xelaev18004582m.g & 2.73 & $2.35 \mathrm{E}-02$ \\
\hline Xelaev18030439m.g & 2.72 & 1.19E-02 \\
\hline scn1b.L & 2.70 & 3.97E-02 \\
\hline magi1.S & 2.70 & $8.65 \mathrm{E}-06$ \\
\hline LOC100489386-like.L & 2.70 & $8.48 \mathrm{E}-04$ \\
\hline pcmtd1.S & 2.69 & $1.64 \mathrm{E}-05$ \\
\hline Xelaev18038420m.g & 2.69 & $4.79 \mathrm{E}-06$ \\
\hline myt1.L & 2.69 & $1.66 \mathrm{E}-04$ \\
\hline tmem198.S & 2.68 & $2.15 \mathrm{E}-07$ \\
\hline ptpru.L & 2.68 & $4.99 \mathrm{E}-05$ \\
\hline fam102b.S & 2.67 & $3.56 \mathrm{E}-02$ \\
\hline pkdcc.S & 2.67 & $1.48 \mathrm{E}-02$ \\
\hline fam222a.L & 2.67 & $1.13 \mathrm{E}-07$ \\
\hline$r \operatorname{tn} 2 . L$ & 2.67 & $2.59 \mathrm{E}-09$ \\
\hline irf1.L & 2.66 & $1.05 \mathrm{E}-02$ \\
\hline fgf8.L & 2.66 & $1.69 \mathrm{E}-02$ \\
\hline tmem55a.S & 2.65 & $1.69 \mathrm{E}-02$ \\
\hline atp8a1.L & 2.65 & $2.15 \mathrm{E}-03$ \\
\hline sst.S & 2.64 & $1.71 \mathrm{E}-02$ \\
\hline twist1.L & 2.64 & $1.31 \mathrm{E}-05$ \\
\hline cables1.L & 2.64 & $4.43 \mathrm{E}-02$ \\
\hline fmn2.L & 2.64 & $1.95 \mathrm{E}-02$ \\
\hline LOC100492639-like.L & 2.63 & $1.93 \mathrm{E}-09$ \\
\hline Xelaev18027769m.g & 2.63 & $3.42 \mathrm{E}-03$ \\
\hline fgd1.S & 2.63 & $1.24 \mathrm{E}-04$ \\
\hline epn1.S & 2.62 & $4.49 \mathrm{E}-06$ \\
\hline slc27a3.L & 2.61 & $1.57 \mathrm{E}-18$ \\
\hline acer2.L & 2.61 & $8.32 \mathrm{E}-06$ \\
\hline btbd11.L & 2.61 & $2.90 \mathrm{E}-02$ \\
\hline hes8.S & 2.60 & $7.63 \mathrm{E}-09$ \\
\hline Irp4.S & 2.60 & $3.56 \mathrm{E}-05$ \\
\hline rcor2.L & 2.60 & $1.35 \mathrm{E}-10$ \\
\hline LOC496795.L & 2.60 & $1.54 \mathrm{E}-03$ \\
\hline
\end{tabular}




\begin{tabular}{|c|c|c|}
\hline tet3.S & 2.59 & 5.99E-05 \\
\hline cdc42se2-like.1.S & 2.58 & $1.73 \mathrm{E}-04$ \\
\hline gdpd5.S & 2.57 & $4.02 \mathrm{E}-05$ \\
\hline rnf125.S & 2.57 & 7.33E-03 \\
\hline spry2.S & 2.57 & $3.58 \mathrm{E}-03$ \\
\hline gnao1.S & 2.56 & 2.07E-06 \\
\hline skida1.S & 2.56 & $5.20 \mathrm{E}-11$ \\
\hline sh2b2.L & 2.56 & 8.47E-03 \\
\hline$n k x 3-1 . L$ & 2.56 & 2.87E-02 \\
\hline rassf2.L & 2.56 & $1.11 \mathrm{E}-04$ \\
\hline gse1.L & 2.56 & $2.46 \mathrm{E}-07$ \\
\hline psat1.S & 2.55 & 4.53E-02 \\
\hline mospd1.L & 2.55 & 2.27E-09 \\
\hline Xetrov90023254m.S & 2.55 & 3.36E-03 \\
\hline enpp2.L & 2.54 & 1.37E-03 \\
\hline pcbp2.S & 2.54 & $8.59 E-05$ \\
\hline cpe.L & 2.54 & $3.04 \mathrm{E}-04$ \\
\hline zc4h2.L & 2.54 & $6.02 \mathrm{E}-07$ \\
\hline ano8.L & 2.53 & $9.38 \mathrm{E}-03$ \\
\hline fam222a.S & 2.53 & 3.37E-03 \\
\hline Irrn1-like.2.L & 2.53 & $2.28 \mathrm{E}-03$ \\
\hline cuedc1.L & 2.51 & 1.57E-04 \\
\hline map1/c3a.L & 2.51 & $1.13 \mathrm{E}-03$ \\
\hline rufy3.L & 2.50 & $8.24 \mathrm{E}-10$ \\
\hline gramd1a.L & 2.49 & $3.43 \mathrm{E}-03$ \\
\hline sh3glb2.S & 2.49 & 1.57E-02 \\
\hline Xelaev18000699m.g & 2.48 & 2.95E-03 \\
\hline onecut2.L & 2.48 & $4.86 \mathrm{E}-03$ \\
\hline chst8.S & 2.48 & $1.78 \mathrm{E}-02$ \\
\hline cass4.L & 2.48 & $2.33 \mathrm{E}-06$ \\
\hline krt19.S & 2.48 & $2.70 \mathrm{E}-04$ \\
\hline a2m.S & 2.47 & $5.92 \mathrm{E}-06$ \\
\hline rnd3.S & 2.45 & $1.40 \mathrm{E}-02$ \\
\hline best2.S & 2.45 & $1.15 \mathrm{E}-02$ \\
\hline epb41/1.L & 2.45 & $5.25 E-05$ \\
\hline Xelaev18031949m.g & 2.44 & $9.04 \mathrm{E}-03$ \\
\hline Ipar4.S & 2.43 & 7.38E-07 \\
\hline Xelaev18021928m.g & 2.43 & $8.13 \mathrm{E}-05$ \\
\hline
\end{tabular}




\begin{tabular}{|c|c|c|}
\hline Xelaev18039965m.g & 2.43 & $4.09 \mathrm{E}-11$ \\
\hline myt1.S & 2.43 & $1.28 \mathrm{E}-05$ \\
\hline hnrnpll.L & 2.43 & $1.39 \mathrm{E}-04$ \\
\hline mob3b.L & 2.42 & $3.12 \mathrm{E}-02$ \\
\hline tox3.L & 2.42 & 3.79E-03 \\
\hline nxpe3.L & 2.42 & $3.24 \mathrm{E}-02$ \\
\hline pik3r3.S & 2.41 & $9.24 \mathrm{E}-05$ \\
\hline efnb2.S & 2.41 & $1.40 \mathrm{E}-03$ \\
\hline rnf125.L & 2.40 & 9.26E-05 \\
\hline Irrc8b.S & 2.40 & $1.44 \mathrm{E}-03$ \\
\hline Xelaev18009525m.g & 2.40 & $1.63 \mathrm{E}-03$ \\
\hline mex3b.S & 2.40 & $2.55 \mathrm{E}-04$ \\
\hline hdac4.L & 2.40 & $2.77 \mathrm{E}-02$ \\
\hline atg9b.L & 2.40 & $1.21 \mathrm{E}-02$ \\
\hline$d 1 \times 6 . S$ & 2.40 & $2.95 \mathrm{E}-03$ \\
\hline Irig3.L & 2.39 & $2.38 \mathrm{E}-06$ \\
\hline clic5.S & 2.38 & $1.18 \mathrm{E}-03$ \\
\hline tet3.L & 2.38 & $3.74 \mathrm{E}-04$ \\
\hline cybrd1.L & 2.38 & $3.13 \mathrm{E}-08$ \\
\hline st3gal2.1.S & 2.38 & $4.18 \mathrm{E}-04$ \\
\hline fyn-like.S & 2.38 & $4.42 \mathrm{E}-03$ \\
\hline$s t \times 2 . L$ & 2.37 & $5.27 \mathrm{E}-05$ \\
\hline fam102a.L & 2.37 & $3.27 \mathrm{E}-05$ \\
\hline dock6-like.L & 2.37 & $1.11 \mathrm{E}-06$ \\
\hline cyp27c1.S & 2.37 & $3.82 \mathrm{E}-04$ \\
\hline LOC100145446.S & 2.37 & $2.84 \mathrm{E}-02$ \\
\hline$m m p 14 . L$ & 2.36 & $3.18 \mathrm{E}-05$ \\
\hline rgs 10.L & 2.36 & $3.31 \mathrm{E}-02$ \\
\hline slc23a2.L & 2.36 & $5.63 \mathrm{E}-05$ \\
\hline myh6.S & 2.36 & $2.75 \mathrm{E}-02$ \\
\hline pknox2.L & 2.36 & $4.31 \mathrm{E}-06$ \\
\hline srrm4.S & 2.35 & $3.69 \mathrm{E}-02$ \\
\hline rimbp2.L & 2.34 & $2.61 \mathrm{E}-02$ \\
\hline tuba1b.L & 2.33 & $7.24 \mathrm{E}-09$ \\
\hline Xetrov90025030m.L & 2.33 & $3.88 \mathrm{E}-03$ \\
\hline map1/c3b.S & 2.32 & $2.45 \mathrm{E}-02$ \\
\hline jag1.L & 2.32 & $9.73 \mathrm{E}-04$ \\
\hline hectd4.L & 2.32 & $1.18 \mathrm{E}-04$ \\
\hline
\end{tabular}




\begin{tabular}{|c|c|c|}
\hline cyyr1.L & 2.31 & 1.02E-02 \\
\hline aplnr.L & 2.31 & 5.80E-07 \\
\hline rufy3.S & 2.30 & 4.99E-02 \\
\hline fam211b.S & 2.30 & $2.37 E-02$ \\
\hline $\operatorname{vim} \cdot L$ & 2.30 & $9.84 \mathrm{E}-07$ \\
\hline rnf130.L & 2.30 & $5.49 \mathrm{E}-03$ \\
\hline kctd1.S & 2.29 & 8.02E-08 \\
\hline epha4.L & 2.28 & $4.47 \mathrm{E}-03$ \\
\hline nkain1.L & 2.28 & $2.36 \mathrm{E}-02$ \\
\hline ephb3.S & 2.27 & 3.76E-09 \\
\hline onecut1.2.S & 2.27 & $3.26 \mathrm{E}-05$ \\
\hline b4galt4.S & 2.26 & $3.90 \mathrm{E}-04$ \\
\hline sept5.L & 2.26 & $8.33 \mathrm{E}-08$ \\
\hline rtbdn.S & 2.26 & $2.34 \mathrm{E}-04$ \\
\hline LOC100489897.L & 2.26 & 4.67E-02 \\
\hline gas1.S & 2.26 & $5.39 \mathrm{E}-04$ \\
\hline$c d c 25 b . L$ & 2.25 & $2.47 \mathrm{E}-04$ \\
\hline LOC100492859.L & 2.25 & $3.77 \mathrm{E}-02$ \\
\hline fnbp1.S & 2.25 & $3.58 \mathrm{E}-03$ \\
\hline smarcc2.S & 2.24 & $2.89 \mathrm{E}-02$ \\
\hline fam43a.L & 2.24 & $1.45 \mathrm{E}-02$ \\
\hline cep85I.S & 2.24 & $2.37 \mathrm{E}-02$ \\
\hline nrarp.L & 2.24 & $2.27 \mathrm{E}-04$ \\
\hline Xelaev18019689m.g & 2.24 & $3.48 \mathrm{E}-02$ \\
\hline Xelaev18016676m.g & 2.24 & $8.81 \mathrm{E}-05$ \\
\hline kctd1.L & 2.24 & $6.53 \mathrm{E}-03$ \\
\hline fam189a1.L & 2.24 & $1.51 \mathrm{E}-02$ \\
\hline map4k4-like.L & 2.23 & $5.45 \mathrm{E}-09$ \\
\hline hmox1.L & 2.23 & $1.01 \mathrm{E}-09$ \\
\hline pcsk5.S & 2.23 & $1.67 \mathrm{E}-03$ \\
\hline sox2.S & 2.22 & $3.18 \mathrm{E}-02$ \\
\hline notch1.L & 2.22 & $1.06 \mathrm{E}-04$ \\
\hline Xelaev18044381m.g & 2.22 & $2.39 \mathrm{E}-06$ \\
\hline Xelaev18016675m.g & 2.20 & $2.30 \mathrm{E}-06$ \\
\hline tubb2b.L & 2.19 & $8.41 \mathrm{E}-04$ \\
\hline bhlhe40.L & 2.19 & 7.01E-04 \\
\hline hes9-2.L & 2.19 & $3.96 \mathrm{E}-02$ \\
\hline bcr.L & 2.18 & $1.90 \mathrm{E}-06$ \\
\hline
\end{tabular}




\begin{tabular}{|c|c|c|}
\hline mylip.S & 2.18 & 1.56E-02 \\
\hline frmd4a.S & 2.18 & $4.40 \mathrm{E}-07$ \\
\hline spire2.S & 2.17 & $2.38 \mathrm{E}-02$ \\
\hline $\operatorname{tacc} 1 . L$ & 2.17 & $3.48 \mathrm{E}-02$ \\
\hline prnp.S & 2.17 & $3.11 \mathrm{E}-03$ \\
\hline dact1.S & 2.17 & 7.08E-03 \\
\hline acy3.L & 2.16 & 7.06E-06 \\
\hline znf850.L & 2.16 & $2.41 \mathrm{E}-03$ \\
\hline cdc42ep4.S & 2.16 & 4.57E-03 \\
\hline hes6.1.S & 2.16 & 3.75E-03 \\
\hline zbtb18.S & 2.15 & 4.19E-02 \\
\hline Xelaev18024012m.g & 2.15 & $8.14 \mathrm{E}-03$ \\
\hline$d k k 1 . S$ & 2.15 & 3.07E-02 \\
\hline Irig3.S & 2.14 & $2.16 \mathrm{E}-02$ \\
\hline tes.L & 2.14 & $1.42 \mathrm{E}-02$ \\
\hline Xelaev18044325m.g & 2.13 & $2.32 \mathrm{E}-05$ \\
\hline p2ry4.L & 2.13 & $4.23 \mathrm{E}-07$ \\
\hline arid1b.S & 2.12 & 4.50E-03 \\
\hline numbl.L & 2.12 & $1.35 \mathrm{E}-02$ \\
\hline gatm.S & 2.12 & 3.17E-02 \\
\hline fgfr1.L & 2.12 & $7.61 \mathrm{E}-09$ \\
\hline marcks.S & 2.12 & $8.29 \mathrm{E}-10$ \\
\hline $\operatorname{lh} \times 2 . L$ & 2.11 & 3.88E-02 \\
\hline Xelaev18028757m.g & 2.11 & 3.94E-02 \\
\hline ppm1e.S & 2.11 & $7.40 \mathrm{E}-03$ \\
\hline cbfb.S & 2.11 & $9.12 \mathrm{E}-06$ \\
\hline mprip.L & 2.11 & $2.06 \mathrm{E}-02$ \\
\hline meis2.L & 2.11 & $2.45 \mathrm{E}-02$ \\
\hline cuedc1.S & 2.10 & $4.17 \mathrm{E}-07$ \\
\hline tmem2.L & 2.10 & 9.24E-05 \\
\hline Xelaev18043719m.g & 2.10 & $1.22 \mathrm{E}-02$ \\
\hline smtn.L & 2.09 & $1.43 \mathrm{E}-03$ \\
\hline$m s i 1 . L$ & 2.09 & $9.71 \mathrm{E}-04$ \\
\hline bach2.S & 2.09 & $2.18 \mathrm{E}-02$ \\
\hline sh3rf1.S & 2.09 & $1.44 \mathrm{E}-04$ \\
\hline rbms1.S & 2.08 & 3.39E-04 \\
\hline atat1.L & 2.08 & 9.96E-06 \\
\hline LOC100493716.S & 2.07 & $1.04 \mathrm{E}-06$ \\
\hline
\end{tabular}




\begin{tabular}{|l|c|c|}
\hline stk40.L & 2.07 & $1.27 \mathrm{E}-03$ \\
\hline Ippr3.S & 2.07 & $3.82 \mathrm{E}-02$ \\
\hline fzd2.L & 2.07 & $2.05 \mathrm{E}-05$ \\
\hline grm7.S & 2.07 & $3.31 \mathrm{E}-02$ \\
\hline lace1.L & 2.06 & $2.47 \mathrm{E}-03$ \\
\hline rftn1.L & 2.05 & $3.27 \mathrm{E}-02$ \\
\hline vegfa.L & 2.05 & $1.38 \mathrm{E}-02$ \\
\hline Xetrov90024734m.L & 2.05 & $2.64 \mathrm{E}-05$ \\
\hline rab11fip4l.S & 2.05 & $2.78 \mathrm{E}-02$ \\
\hline vav2.S & 2.05 & $3.75 \mathrm{E}-04$ \\
\hline Xelaev18010400m.g & 2.05 & $1.85 \mathrm{E}-02$ \\
\hline tspan17.S & 2.05 & $2.51 \mathrm{E}-06$ \\
\hline sesn1.S & 2.04 & $8.95 \mathrm{E}-07$ \\
\hline tiparp.L & 2.04 & $1.35 \mathrm{E}-04$ \\
\hline LOC100489393.L & 2.04 & $4.10 \mathrm{E}-03$ \\
\hline hes5.1.S & 2.03 & $5.25 \mathrm{E}-03$ \\
\hline fsd1.L & 2.03 & $8.36 \mathrm{E}-04$ \\
\hline ankrd10.L & 2.02 & $4.16 \mathrm{E}-04$ \\
\hline paqr4-like.S & 2.02 & $3.36 \mathrm{E}-02$ \\
\hline irx2.L & 2.01 & $4.57 \mathrm{E}-05$ \\
\hline draxin.S & 2.01 & $2.68 \mathrm{E}-02$ \\
\hline fgfr4.S & 2.00 & $6.94 \mathrm{E}-10$ \\
\hline dock4.L & $5.02 \mathrm{E}-03$ \\
\hline amotl2.S & $4.42 \mathrm{E}-03$ \\
\hline
\end{tabular}

Table S19: Summary of differentially expressed genes by Ptf1a + Brg1MO

\begin{tabular}{|l|c|c|}
\hline ID & log2FC & P-value \\
\hline Xelaev18007508m.g & 12.92 & $2.82 \mathrm{E}-14$ \\
\hline neurod4.L & 11.64 & $2.14 \mathrm{E}-11$ \\
\hline prdm13.L & 10.92 & $5.72 \mathrm{E}-11$ \\
\hline pnhd.S & 10.65 & $3.20 \mathrm{E}-08$ \\
\hline prph.S & 10.55 & $2.20 \mathrm{E}-20$ \\
\hline neurod4.S & 10.49 & $1.02 \mathrm{E}-08$ \\
\hline prdm13.S & 10.45 & $2.57 \mathrm{E}-15$ \\
\hline scrt1.S & 9.99 & $1.73 \mathrm{E}-09$ \\
\hline pou3f1.L & 9.59 & $7.06 \mathrm{E}-08$ \\
\hline
\end{tabular}




\begin{tabular}{|c|c|c|}
\hline prdm8.L & 9.58 & $6.76 \mathrm{E}-04$ \\
\hline neurog1.L & 9.55 & $2.85 \mathrm{E}-08$ \\
\hline igfbp/1.L & 9.37 & $1.21 \mathrm{E}-07$ \\
\hline dpys/4.L & 9.08 & $7.21 \mathrm{E}-07$ \\
\hline fezf1.L & 9.05 & 4.24E-05 \\
\hline eva1b.S & 9.00 & 1.57E-06 \\
\hline st18.L & 8.95 & $3.86 \mathrm{E}-07$ \\
\hline LOC100490531-like.L & 8.84 & 2.66E-07 \\
\hline Xetrov90027705m.L & 8.63 & $6.53 \mathrm{E}-12$ \\
\hline des.2.L & 8.59 & 9.57E-06 \\
\hline foxd2.L & 8.58 & 5.57E-05 \\
\hline shd.L & 8.43 & $2.83 E-11$ \\
\hline$e b f 2 . L$ & 8.41 & $1.15 \mathrm{E}-23$ \\
\hline slc26a9.S & 8.36 & 2.91E-05 \\
\hline hes5.2.L & 8.30 & $4.58 \mathrm{E}-05$ \\
\hline pyy.L & 8.25 & $2.52 \mathrm{E}-05$ \\
\hline aldh1b1.L & 8.16 & $8.16 \mathrm{E}-05$ \\
\hline tfap2b.S & 8.14 & $1.39 \mathrm{E}-28$ \\
\hline LOC100496628-like.L & 8.06 & $4.50 \mathrm{E}-05$ \\
\hline slc18a3.S & 8.05 & $3.01 \mathrm{E}-05$ \\
\hline tespa1.S & 8.02 & 7.93E-05 \\
\hline olig3.S & 8.01 & $1.22 \mathrm{E}-03$ \\
\hline Xelaev18032183m.g & 7.98 & $3.98 \mathrm{E}-05$ \\
\hline tubb3.L & 7.94 & 8.95E-06 \\
\hline shox2.L & 7.91 & $9.14 \mathrm{E}-05$ \\
\hline skor1.S & 7.86 & $2.10 \mathrm{E}-08$ \\
\hline Xelaev18047032m.g & 7.84 & $1.42 \mathrm{E}-03$ \\
\hline adcyap1.S & 7.81 & $1.29 \mathrm{E}-04$ \\
\hline$s p 9 . L$ & 7.79 & $1.47 \mathrm{E}-02$ \\
\hline tfap2e.L & 7.68 & $2.89 \mathrm{E}-05$ \\
\hline des.1.L & 7.63 & $3.06 \mathrm{E}-08$ \\
\hline chat.S & 7.58 & $1.39 \mathrm{E}-03$ \\
\hline sag.S & 7.58 & $2.40 \mathrm{E}-03$ \\
\hline admp.L & 7.57 & $1.65 \mathrm{E}-02$ \\
\hline fam212b.L & 7.55 & $7.85 \mathrm{E}-04$ \\
\hline ptf1a.L & 7.55 & $1.07 \mathrm{E}-04$ \\
\hline nhlh1.L & 7.51 & $3.24 \mathrm{E}-05$ \\
\hline Xelaev18000445m.g & 7.51 & $2.94 \mathrm{E}-04$ \\
\hline
\end{tabular}




\begin{tabular}{|c|c|c|}
\hline Xelaev18018357m.g & 7.49 & $3.84 \mathrm{E}-04$ \\
\hline slc46a2.L & 7.45 & $1.51 \mathrm{E}-02$ \\
\hline kirrel2.L & 7.44 & $5.77 \mathrm{E}-86$ \\
\hline hoxd1.S & 7.44 & 1.44E-03 \\
\hline egr4.L & 7.43 & $3.14 \mathrm{E}-03$ \\
\hline dpys/3.L & 7.36 & $5.79 \mathrm{E}-62$ \\
\hline mllt11.S & 7.31 & $2.71 \mathrm{E}-04$ \\
\hline pnhd.L & 7.15 & $4.38 \mathrm{E}-08$ \\
\hline bgn.L & 7.10 & $4.49 \mathrm{E}-38$ \\
\hline aqp3.L & 7.07 & $9.68 \mathrm{E}-03$ \\
\hline nr5a2.L & 7.06 & $7.56 \mathrm{E}-04$ \\
\hline ebf2.S & 7.05 & $2.59 \mathrm{E}-21$ \\
\hline Ibx1.S & 7.04 & $3.03 E-04$ \\
\hline barh/2.L & 6.99 & $9.12 \mathrm{E}-04$ \\
\hline hesx1.S & 6.97 & $1.83 \mathrm{E}-06$ \\
\hline sybu.L & 6.97 & $3.28 \mathrm{E}-06$ \\
\hline Xelaev18040877m.g & 6.96 & $1.47 \mathrm{E}-14$ \\
\hline prdm8.S & 6.95 & $4.51 \mathrm{E}-03$ \\
\hline vsx1.S & 6.93 & $2.12 \mathrm{E}-03$ \\
\hline pcdh9.L & 6.89 & $1.08 \mathrm{E}-03$ \\
\hline elavl3.S & 6.86 & $6.30 \mathrm{E}-04$ \\
\hline$n p t \times 2 . L$ & 6.84 & $1.89 \mathrm{E}-02$ \\
\hline clstn3.L & 6.84 & $4.64 \mathrm{E}-09$ \\
\hline shox2.S & 6.84 & $1.07 \mathrm{E}-03$ \\
\hline Xetrov90027705m.S & 6.83 & $1.41 \mathrm{E}-03$ \\
\hline kiaa1755.L & 6.82 & $3.89 \mathrm{E}-11$ \\
\hline Xelaev18046363m.g & 6.82 & $1.42 \mathrm{E}-02$ \\
\hline map2.S & 6.80 & $4.04 \mathrm{E}-28$ \\
\hline tapbp.L & 6.79 & $8.13 \mathrm{E}-04$ \\
\hline ptger3.L & 6.74 & $1.78 \mathrm{E}-03$ \\
\hline LOC100490531-like.S & 6.74 & $3.97 \mathrm{E}-03$ \\
\hline hspb8.L & 6.73 & $2.82 \mathrm{E}-02$ \\
\hline nhlh1.S & 6.71 & $4.78 \mathrm{E}-04$ \\
\hline barhl2.S & 6.70 & $3.51 \mathrm{E}-06$ \\
\hline$m n \times 1 . L$ & 6.69 & $9.01 \mathrm{E}-03$ \\
\hline tcf15.S & 6.65 & $4.71 \mathrm{E}-03$ \\
\hline gad1.1.L & 6.63 & $1.47 \mathrm{E}-11$ \\
\hline$n k \times 3-2 . S$ & 6.63 & $2.69 \mathrm{E}-02$ \\
\hline
\end{tabular}




\begin{tabular}{|c|c|c|}
\hline spry4.S & 6.60 & $1.06 \mathrm{E}-02$ \\
\hline Xelaev18045246m.g & 6.60 & 4.97E-03 \\
\hline admp.S & 6.60 & 4.95E-02 \\
\hline skor2.S & 6.59 & 1.96E-02 \\
\hline myod1.S & 6.57 & $1.83 \mathrm{E}-03$ \\
\hline kirrel2.S & 6.54 & $5.79 \mathrm{E}-62$ \\
\hline$h m \times 1.1$ & 6.50 & 3.13E-02 \\
\hline Sox17a.L & 6.49 & $1.35 \mathrm{E}-03$ \\
\hline Xelaev18033867m.g & 6.42 & $1.40 \mathrm{E}-02$ \\
\hline Xelaev18020334m.g & 6.39 & $2.71 \mathrm{E}-03$ \\
\hline Xelaev18036651m.g & 6.28 & $2.51 \mathrm{E}-24$ \\
\hline myo3b.L & 6.23 & 1.67E-02 \\
\hline tmem116.L & 6.22 & $4.53 \mathrm{E}-12$ \\
\hline$m m p 17 . L$ & 6.21 & 3.34E-03 \\
\hline hes6.2.L & 6.21 & 4.19E-02 \\
\hline nova2.S & 6.18 & $6.24 \mathrm{E}-03$ \\
\hline nes. $L$ & 6.16 & $1.76 \mathrm{E}-10$ \\
\hline sostdc1.S & 6.15 & $1.45 \mathrm{E}-02$ \\
\hline$m m p 28 . L$ & 6.12 & 3.62E-02 \\
\hline neurod1.L & 6.11 & $2.89 \mathrm{E}-02$ \\
\hline ror2.S & 6.09 & $2.63 \mathrm{E}-17$ \\
\hline Imo2.S & 6.08 & $1.91 \mathrm{E}-03$ \\
\hline Xelaev18034787m.g & 6.08 & $1.57 \mathrm{E}-02$ \\
\hline pla2g12b.S & 6.07 & $1.06 \mathrm{E}-02$ \\
\hline Irrn1-like.1.S & 6.06 & $9.05 \mathrm{E}-32$ \\
\hline Xelaev18042973m.g & 6.01 & $2.14 \mathrm{E}-04$ \\
\hline chst3.L & 5.96 & $5.95 \mathrm{E}-10$ \\
\hline cdh1.L & 5.94 & $1.53 \mathrm{E}-08$ \\
\hline tmem47.L & 5.93 & $2.47 \mathrm{E}-02$ \\
\hline Xelaev18026532m.g & 5.90 & $1.35 \mathrm{E}-02$ \\
\hline sp5.L & 5.88 & $8.84 \mathrm{E}-03$ \\
\hline matn4.S & 5.88 & $2.25 \mathrm{E}-02$ \\
\hline LOC100495096.1 & 5.87 & $1.01 \mathrm{E}-02$ \\
\hline chrd.S & 5.85 & $4.11 \mathrm{E}-03$ \\
\hline bmp3.L & 5.82 & $2.82 \mathrm{E}-02$ \\
\hline igf2.L & 5.78 & $7.57 \mathrm{E}-04$ \\
\hline Xelaev18035158m.g & 5.76 & $4.19 \mathrm{E}-02$ \\
\hline olig2.S & 5.74 & $2.57 \mathrm{E}-16$ \\
\hline
\end{tabular}




\begin{tabular}{|c|c|c|}
\hline usp13.S & 5.71 & $1.90 \mathrm{E}-03$ \\
\hline LOC100489771.L & 5.70 & $3.60 \mathrm{E}-02$ \\
\hline $\operatorname{limd2.L}$ & 5.67 & $3.98 \mathrm{E}-20$ \\
\hline tubb3.S & 5.67 & $2.86 \mathrm{E}-21$ \\
\hline$m \times r a 7 . L$ & 5.67 & $4.50 \mathrm{E}-19$ \\
\hline Xelaev18004302m.g & 5.65 & $1.53 \mathrm{E}-02$ \\
\hline map3k13.L & 5.63 & 3.05E-02 \\
\hline fam198b.L & 5.62 & $1.56 \mathrm{E}-03$ \\
\hline plekhd1.L & 5.61 & 1.23E-02 \\
\hline neurog2.S & 5.54 & $1.93 \mathrm{E}-03$ \\
\hline neurod1.S & 5.50 & 4.96E-02 \\
\hline neurog2.L & 5.50 & $3.42 \mathrm{E}-02$ \\
\hline dmrta2.L & 5.49 & $6.01 \mathrm{E}-03$ \\
\hline hoxc3.L & 5.49 & $2.77 \mathrm{E}-02$ \\
\hline $\ln x 8 . L$ & 5.47 & $1.51 \mathrm{E}-02$ \\
\hline hortr2.L & 5.42 & $2.46 \mathrm{E}-02$ \\
\hline nova1.L & 5.41 & $1.10 \mathrm{E}-11$ \\
\hline wnt8a.L & 5.40 & $2.82 \mathrm{E}-03$ \\
\hline hes5_X2.L & 5.40 & $5.35 \mathrm{E}-08$ \\
\hline Xelaev18025499m.g & 5.39 & $1.32 \mathrm{E}-04$ \\
\hline$n k x 2-6.5$ & 5.36 & $1.14 \mathrm{E}-05$ \\
\hline dpp10.S & 5.35 & $8.39 \mathrm{E}-16$ \\
\hline Xelaev18015119m.g & 5.33 & 3.04E-06 \\
\hline$t / x 3 . L$ & 5.31 & $6.78 \mathrm{E}-04$ \\
\hline$z e b 2 . L$ & 5.28 & $2.34 \mathrm{E}-13$ \\
\hline LOC101730771-like.L & 5.28 & $4.13 \mathrm{E}-04$ \\
\hline sez6.S & 5.26 & $9.88 \mathrm{E}-05$ \\
\hline$z e b 2 . S$ & 5.25 & $1.47 \mathrm{E}-14$ \\
\hline LOC100487395.L & 5.25 & $1.90 \mathrm{E}-23$ \\
\hline six1.S & 5.24 & $1.33 \mathrm{E}-02$ \\
\hline bhlhe22.L & 5.23 & $4.68 \mathrm{E}-02$ \\
\hline elav/4.L & 5.22 & $2.73 \mathrm{E}-07$ \\
\hline fam212a.L & 5.22 & $1.47 \mathrm{E}-13$ \\
\hline tub.L & 5.22 & $2.88 \mathrm{E}-02$ \\
\hline spns2.L & 5.20 & $2.31 \mathrm{E}-02$ \\
\hline Xelaev18026630m.g & 5.20 & $1.88 \mathrm{E}-05$ \\
\hline gpr63.S & 5.19 & $1.81 \mathrm{E}-07$ \\
\hline$n m b . S$ & 5.17 & $1.55 \mathrm{E}-03$ \\
\hline
\end{tabular}




\begin{tabular}{|c|c|c|}
\hline tal1.L & 5.14 & 7.85E-04 \\
\hline faxc.S & 5.11 & $1.85 \mathrm{E}-03$ \\
\hline skor2.L & 5.10 & 5.37E-04 \\
\hline$n p r 3 . L$ & 5.09 & 8.67E-03 \\
\hline onecut1.2.L & 5.08 & $5.74 \mathrm{E}-13$ \\
\hline stk32a.L & 5.06 & 3.08E-02 \\
\hline pax2.L & 5.06 & $6.42 \mathrm{E}-05$ \\
\hline ppp1r9b.S & 5.03 & $1.33 \mathrm{E}-03$ \\
\hline nes.S & 5.00 & $6.41 \mathrm{E}-12$ \\
\hline slc32a1.S & 4.94 & 4.98E-02 \\
\hline hes5_X1.L & 4.92 & 4.89E-02 \\
\hline$c p / x 2 . L$ & 4.92 & $8.77 \mathrm{E}-12$ \\
\hline phlda1.L & 4.91 & 6.03E-05 \\
\hline LOC100492420.L & 4.91 & $3.43 \mathrm{E}-02$ \\
\hline Sox9.L & 4.88 & $3.30 \mathrm{E}-02$ \\
\hline sall3.L & 4.87 & $1.51 \mathrm{E}-07$ \\
\hline adcyap1.L & 4.86 & $1.90 \mathrm{E}-03$ \\
\hline tal1.S & 4.86 & 1.96E-03 \\
\hline Xelaev18003346m.g & 4.85 & $9.53 \mathrm{E}-05$ \\
\hline map3k12.S & 4.85 & $2.66 \mathrm{E}-03$ \\
\hline tfap2b.L & 4.85 & $2.40 \mathrm{E}-13$ \\
\hline map3k12.L & 4.85 & $2.42 \mathrm{E}-05$ \\
\hline$d m b \times 1 . L$ & 4.82 & $8.47 \mathrm{E}-05$ \\
\hline eda2r.L & 4.79 & $2.63 \mathrm{E}-17$ \\
\hline crabp2.S & 4.78 & $5.95 \mathrm{E}-10$ \\
\hline$m c f 2 I . S$ & 4.78 & $6.86 \mathrm{E}-08$ \\
\hline nt5e.S & 4.76 & $4.56 \mathrm{E}-02$ \\
\hline cacna2d1.L & 4.74 & $1.02 \mathrm{E}-04$ \\
\hline Xelaev18007001m.g & 4.74 & $5.35 \mathrm{E}-11$ \\
\hline LOC100494774.L & 4.74 & $2.75 \mathrm{E}-02$ \\
\hline des.1.S & 4.72 & $7.11 \mathrm{E}-07$ \\
\hline Xelaev18005831m.g & 4.72 & $1.18 \mathrm{E}-04$ \\
\hline Xetrov90000859m.L & 4.72 & $1.36 \mathrm{E}-06$ \\
\hline mycl.S & 4.71 & $2.08 \mathrm{E}-13$ \\
\hline vegt.S & 4.70 & $4.58 \mathrm{E}-03$ \\
\hline arx.L & 4.70 & $4.66 \mathrm{E}-02$ \\
\hline stxbp1.S & 4.69 & 2.67E-02 \\
\hline gcnt1.S & 4.69 & $1.18 \mathrm{E}-08$ \\
\hline
\end{tabular}




\begin{tabular}{|c|c|c|}
\hline Xelaev18016582m.g & 4.68 & $1.07 \mathrm{E}-15$ \\
\hline zic3.S & 4.68 & $1.65 \mathrm{E}-02$ \\
\hline hdac9.S & 4.68 & $2.34 \mathrm{E}-02$ \\
\hline LOC100487395.S & 4.65 & $8.27 \mathrm{E}-07$ \\
\hline s/c38a8.L & 4.65 & 7.49E-03 \\
\hline LOC100492804.S & 4.64 & 9.94E-03 \\
\hline bhlhe22.S & 4.64 & $1.30 \mathrm{E}-02$ \\
\hline plekhg4.S & 4.64 & $1.50 \mathrm{E}-06$ \\
\hline ankrd65.L & 4.62 & $4.21 \mathrm{E}-08$ \\
\hline pax2.S & 4.62 & $2.21 \mathrm{E}-08$ \\
\hline efemp2.L & 4.60 & $4.15 \mathrm{E}-02$ \\
\hline$m y / 1 . L$ & 4.59 & $4.76 \mathrm{E}-02$ \\
\hline wipf1.L & 4.55 & 4.09E-04 \\
\hline rufy2.L & 4.53 & 8.98E-04 \\
\hline skor1.L & 4.53 & $1.04 \mathrm{E}-03$ \\
\hline insm1.L & 4.51 & $1.76 \mathrm{E}-02$ \\
\hline hes5_X2.S & 4.48 & $2.79 \mathrm{E}-03$ \\
\hline runx1t1.S & 4.48 & 3.74E-07 \\
\hline mtmr7-like.L & 4.47 & $2.51 \mathrm{E}-04$ \\
\hline LOC100487796-like.L & 4.46 & $6.78 \mathrm{E}-16$ \\
\hline faxc.L & 4.46 & $3.63 \mathrm{E}-11$ \\
\hline lef1.S & 4.43 & 2.07E-05 \\
\hline celf2.L & 4.43 & 4.34E-09 \\
\hline asb2.L & 4.42 & $5.32 \mathrm{E}-03$ \\
\hline pck1.L & 4.42 & $8.35 \mathrm{E}-08$ \\
\hline dlx6.L & 4.41 & $2.44 \mathrm{E}-14$ \\
\hline Xelaev18038044m.g & 4.39 & $5.84 \mathrm{E}-03$ \\
\hline gfra1.S & 4.39 & $7.78 \mathrm{E}-11$ \\
\hline adora2a.L & 4.36 & $3.28 \mathrm{E}-02$ \\
\hline nyx.S & 4.35 & $1.84 \mathrm{E}-02$ \\
\hline CXCr2.L & 4.35 & $9.45 \mathrm{E}-03$ \\
\hline Xetrov90016928m.L & 4.34 & $8.02 \mathrm{E}-03$ \\
\hline klf7.L & 4.32 & 5.92E-03 \\
\hline$c f d . L$ & 4.32 & $2.60 \mathrm{E}-02$ \\
\hline foxd3.L & 4.31 & $2.92 \mathrm{E}-02$ \\
\hline sema3d.L & 4.31 & $1.04 \mathrm{E}-04$ \\
\hline kif26a.S & 4.29 & $4.64 \mathrm{E}-09$ \\
\hline Xelaev18030284m.g & 4.28 & $7.28 \mathrm{E}-05$ \\
\hline
\end{tabular}




\begin{tabular}{|c|c|c|}
\hline serpinf1.L & 4.27 & $2.74 \mathrm{E}-05$ \\
\hline pitx2.L & 4.27 & 5.05E-03 \\
\hline Xetrov90008930m.S & 4.23 & $1.51 \mathrm{E}-02$ \\
\hline$d b n 1 . S$ & 4.22 & $7.23 \mathrm{E}-14$ \\
\hline spon1.S & 4.21 & $5.16 \mathrm{E}-05$ \\
\hline gad1.1.S & 4.21 & 4.56E-03 \\
\hline Xelaev18042154m.g & 4.20 & 4.48E-07 \\
\hline Xelaev18002611m.g & 4.20 & 1.23E-02 \\
\hline snap25.L & 4.20 & $5.96 \mathrm{E}-13$ \\
\hline Xelaev18024617m.g & 4.19 & $3.74 \mathrm{E}-07$ \\
\hline cbfa2t2.L & 4.18 & $5.24 \mathrm{E}-12$ \\
\hline $\operatorname{lh} \times 5 . L$ & 4.16 & 1.53E-02 \\
\hline tmeff1.S & 4.14 & $1.50 \mathrm{E}-06$ \\
\hline hes5.3.S & 4.14 & $2.78 \mathrm{E}-03$ \\
\hline sncb.L & 4.14 & $2.09 \mathrm{E}-10$ \\
\hline$d b n 1 . L$ & 4.12 & $1.96 \mathrm{E}-17$ \\
\hline itga2b.1-like.S & 4.12 & $2.10 \mathrm{E}-06$ \\
\hline c8orf46.S & 4.12 & $4.41 \mathrm{E}-03$ \\
\hline st8sia6.L & 4.11 & $1.94 \mathrm{E}-02$ \\
\hline Xelaev18021728m.g & 4.10 & $1.23 \mathrm{E}-07$ \\
\hline gngt2.1.S & 4.09 & $2.28 \mathrm{E}-02$ \\
\hline aldh1a2.L & 4.08 & 9.73E-06 \\
\hline bcam.S & 4.08 & $1.60 \mathrm{E}-04$ \\
\hline dnajc6.S & 4.06 & $1.66 \mathrm{E}-05$ \\
\hline Xelaev18018196m.g & 4.06 & 3.16E-04 \\
\hline LOC100489305.S & 4.06 & $4.89 \mathrm{E}-02$ \\
\hline kremen2.S & 4.06 & $2.14 \mathrm{E}-04$ \\
\hline plekho1.L & 4.05 & $3.03 E-04$ \\
\hline hes7.L & 4.04 & $1.21 \mathrm{E}-02$ \\
\hline$t b \times 6 . L$ & 4.04 & $1.04 \mathrm{E}-03$ \\
\hline crabp2.L & 4.04 & $3.56 \mathrm{E}-08$ \\
\hline hes8.L & 4.03 & $1.15 \mathrm{E}-13$ \\
\hline hoxd1.L & 4.03 & $2.83 \mathrm{E}-03$ \\
\hline Xelaev18025917m.g & 4.02 & $7.63 \mathrm{E}-05$ \\
\hline$d m b x 1 . S$ & 4.01 & $1.99 \mathrm{E}-03$ \\
\hline gfi1.L & 4.00 & $3.73 \mathrm{E}-03$ \\
\hline crybb3.L & 4.00 & $5.61 \mathrm{E}-03$ \\
\hline mamdc2.L & 3.99 & 9.01E-03 \\
\hline
\end{tabular}




\begin{tabular}{|c|c|c|}
\hline asb1.L & 3.98 & 3.36E-10 \\
\hline Xelaev18039076m.g & 3.98 & $1.89 \mathrm{E}-13$ \\
\hline hdac7.S & 3.97 & $5.99 \mathrm{E}-04$ \\
\hline nrp2.L & 3.97 & $6.14 \mathrm{E}-10$ \\
\hline rab3b.S & 3.95 & $6.48 \mathrm{E}-03$ \\
\hline sacs.L & 3.95 & $1.33 \mathrm{E}-03$ \\
\hline mecom.L & 3.92 & $6.80 \mathrm{E}-03$ \\
\hline nox4.S & 3.92 & 2.27E-03 \\
\hline st6galnac6.L & 3.87 & 1.87E-02 \\
\hline gli2.S & 3.87 & $1.29 \mathrm{E}-06$ \\
\hline cpe.S & 3.84 & $9.43 \mathrm{E}-06$ \\
\hline pde10a.L & 3.84 & $9.58 \mathrm{E}-03$ \\
\hline fn1.S & 3.83 & $1.41 \mathrm{E}-20$ \\
\hline zc3h12c.L & 3.82 & $2.44 \mathrm{E}-14$ \\
\hline gipr.L & 3.82 & 4.09E-02 \\
\hline tmcc2.S & 3.81 & $3.08 \mathrm{E}-02$ \\
\hline zcchc24.L & 3.80 & $1.62 \mathrm{E}-02$ \\
\hline crmp1.L & 3.80 & $1.49 \mathrm{E}-02$ \\
\hline fstl1.S & 3.79 & $3.13 \mathrm{E}-05$ \\
\hline dennd2c.L & 3.78 & $1.18 \mathrm{E}-10$ \\
\hline st3gal2.2.L & 3.78 & $2.08 \mathrm{E}-02$ \\
\hline clip2.S & 3.77 & $5.35 \mathrm{E}-03$ \\
\hline manea.L & 3.77 & $2.06 \mathrm{E}-05$ \\
\hline ssbp4.S & 3.75 & $1.62 \mathrm{E}-02$ \\
\hline plekhg4.L & 3.73 & $4.11 \mathrm{E}-11$ \\
\hline Sox11.L & 3.72 & $6.24 \mathrm{E}-03$ \\
\hline ulk1.L & 3.72 & 5.04E-09 \\
\hline $\operatorname{lh} \times 5 . S$ & 3.71 & $2.72 \mathrm{E}-06$ \\
\hline pmp22.L & 3.70 & $2.41 \mathrm{E}-06$ \\
\hline st18.S & 3.69 & $3.58 \mathrm{E}-03$ \\
\hline tuba4a.L & 3.67 & $1.47 \mathrm{E}-12$ \\
\hline Xelaev18008189m.g & 3.67 & $1.77 \mathrm{E}-04$ \\
\hline ror2.L & 3.66 & $4.64 \mathrm{E}-09$ \\
\hline srrm4.L & 3.65 & $9.79 \mathrm{E}-04$ \\
\hline insm2.L & 3.65 & $1.19 \mathrm{E}-04$ \\
\hline cd82.L & 3.64 & $1.10 \mathrm{E}-08$ \\
\hline jam3.S & 3.64 & $3.63 \mathrm{E}-03$ \\
\hline$n r 2 f 2 . L$ & 3.63 & $2.48 \mathrm{E}-02$ \\
\hline
\end{tabular}




\begin{tabular}{|c|c|c|}
\hline dennd2c.S & 3.63 & $4.25 \mathrm{E}-08$ \\
\hline cyp1c1.L & 3.62 & 3.99E-02 \\
\hline cbfa2t2.S & 3.62 & $7.57 \mathrm{E}-11$ \\
\hline ppp1r9b.L & 3.61 & $3.61 \mathrm{E}-03$ \\
\hline LOC101734175.L & 3.60 & $4.55 \mathrm{E}-02$ \\
\hline cacna2d2.L & 3.59 & $1.83 E-03$ \\
\hline artn.S & 3.58 & $2.02 \mathrm{E}-02$ \\
\hline dnajc6.L & 3.55 & $6.61 \mathrm{E}-06$ \\
\hline $\operatorname{Irp4.L}$ & 3.54 & 4.79E-07 \\
\hline elav/3.L & 3.54 & $1.80 \mathrm{E}-14$ \\
\hline runx1t1.L & 3.53 & $1.09 \mathrm{E}-03$ \\
\hline Xelaev18043580m.g & 3.53 & $6.95 \mathrm{E}-13$ \\
\hline hes10.L & 3.53 & $8.68 \mathrm{E}-06$ \\
\hline $\operatorname{limd2.S}$ & 3.52 & 3.03E-03 \\
\hline tox.L & 3.52 & $4.70 \mathrm{E}-05$ \\
\hline eln2.L & 3.51 & $2.99 \mathrm{E}-02$ \\
\hline cdk5r2.L & 3.51 & $3.35 \mathrm{E}-02$ \\
\hline$m d k \cdot L$ & 3.51 & $7.13 \mathrm{E}-15$ \\
\hline vim.S & 3.50 & $1.66 \mathrm{E}-02$ \\
\hline hes2.L & 3.49 & $1.27 \mathrm{E}-03$ \\
\hline$m t c / 1 . L$ & 3.48 & $4.41 \mathrm{E}-04$ \\
\hline Xelaev18044438m.g & 3.45 & $9.17 \mathrm{E}-04$ \\
\hline tmem35.L & 3.44 & 1.84E-04 \\
\hline sp5I.S & 3.43 & $2.26 \mathrm{E}-03$ \\
\hline LOC100498368-like.L & 3.43 & $3.42 \mathrm{E}-03$ \\
\hline zfhx $x$.S & 3.42 & $9.24 \mathrm{E}-10$ \\
\hline Irrn1-like.1.L & 3.41 & $9.20 \mathrm{E}-12$ \\
\hline cyyr1.L & 3.40 & $2.37 \mathrm{E}-05$ \\
\hline myh6.L & 3.39 & $2.66 \mathrm{E}-03$ \\
\hline adamts/1.L & 3.38 & $4.08 \mathrm{E}-06$ \\
\hline aldh1a2.S & 3.37 & $4.12 \mathrm{E}-09$ \\
\hline mcf2I.2.L & 3.36 & $1.60 \mathrm{E}-05$ \\
\hline rgmb.L & 3.36 & $1.63 \mathrm{E}-03$ \\
\hline$a b c b 9 . L$ & 3.36 & $9.36 \mathrm{E}-07$ \\
\hline cttnbp2nl.L & 3.36 & $2.25 \mathrm{E}-04$ \\
\hline Ippr3.S & 3.36 & $8.24 \mathrm{E}-05$ \\
\hline Xelaev18029662m.g & 3.35 & $1.06 \mathrm{E}-02$ \\
\hline slco5a1.S & 3.35 & $4.80 \mathrm{E}-04$ \\
\hline
\end{tabular}




\begin{tabular}{|c|c|c|}
\hline Irfn1.S & 3.33 & 3.98E-02 \\
\hline LOC101732555.L & 3.33 & $2.16 \mathrm{E}-02$ \\
\hline arl8a.S & 3.32 & $1.99 \mathrm{E}-03$ \\
\hline pak3.L & 3.32 & 8.96E-03 \\
\hline gli2.L & 3.31 & $1.45 \mathrm{E}-10$ \\
\hline LOC105946937.L & 3.31 & $4.75 \mathrm{E}-05$ \\
\hline tfap2e.S & 3.31 & $1.50 \mathrm{E}-05$ \\
\hline gria1-like.1.S & 3.30 & $6.72 \mathrm{E}-04$ \\
\hline Xelaev18012713m.g & 3.29 & $1.15 \mathrm{E}-02$ \\
\hline Xelaev18007062m.g & 3.29 & $5.12 \mathrm{E}-07$ \\
\hline evi5.L & 3.27 & $3.05 E-16$ \\
\hline zic3.L & 3.27 & $2.06 \mathrm{E}-02$ \\
\hline LOC100490436.L & 3.27 & $3.08 \mathrm{E}-02$ \\
\hline Xelaev18047968m.g & 3.26 & 3.83E-02 \\
\hline Xelaev18004582m.g & 3.26 & $3.55 \mathrm{E}-03$ \\
\hline rassf6.L & 3.26 & $4.08 \mathrm{E}-03$ \\
\hline rgs3.L & 3.26 & $6.20 \mathrm{E}-03$ \\
\hline Xelaev18032990m.g & 3.24 & $3.80 \mathrm{E}-02$ \\
\hline mxi1.S & 3.24 & $2.94 \mathrm{E}-04$ \\
\hline Xelaev18001443m.g & 3.24 & $1.66 \mathrm{E}-03$ \\
\hline ulk1.S & 3.24 & $4.05 E-08$ \\
\hline sgip1.L & 3.23 & $2.68 \mathrm{E}-05$ \\
\hline Xelaev18027950m.g & 3.23 & $6.77 \mathrm{E}-10$ \\
\hline sox11.S & 3.22 & $2.70 \mathrm{E}-02$ \\
\hline sesn1.L & 3.21 & $9.96 \mathrm{E}-06$ \\
\hline hapln3.S & 3.20 & $4.31 \mathrm{E}-03$ \\
\hline btbd19.S & 3.20 & 1.19E-02 \\
\hline arid1b.L & 3.18 & $2.21 \mathrm{E}-12$ \\
\hline Xelaev18028200m.g & 3.17 & $4.85 \mathrm{E}-02$ \\
\hline pax8.L & 3.17 & $2.84 \mathrm{E}-03$ \\
\hline cmtm4.S & 3.17 & $2.33 \mathrm{E}-02$ \\
\hline unc $45 b . S$ & 3.16 & $2.15 \mathrm{E}-06$ \\
\hline eya1.S & 3.16 & $3.86 \mathrm{E}-06$ \\
\hline kif26a.L & 3.15 & $8.12 \mathrm{E}-04$ \\
\hline Irpprc.S & 3.15 & $5.54 \mathrm{E}-04$ \\
\hline rnf165.L & 3.15 & 4.89E-03 \\
\hline LOC100496628-like.S & 3.13 & $5.26 \mathrm{E}-04$ \\
\hline$x k r 4 . L$ & 3.12 & 3.03E-05 \\
\hline
\end{tabular}




\begin{tabular}{|c|c|c|}
\hline pip4k2b.L & 3.12 & $1.35 \mathrm{E}-08$ \\
\hline foxf1.L & 3.11 & $2.18 \mathrm{E}-02$ \\
\hline cdc42ep3.S & 3.11 & $1.25 \mathrm{E}-04$ \\
\hline$d / I 1 . L$ & 3.11 & 4.08E-05 \\
\hline pak3.S & 3.10 & $1.74 \mathrm{E}-08$ \\
\hline pole3.S & 3.09 & $2.23 E-02$ \\
\hline auts2.S & 3.09 & 8.08E-04 \\
\hline st3gal5.S & 3.09 & $2.49 \mathrm{E}-02$ \\
\hline b4galnt1.S & 3.07 & 3.39E-04 \\
\hline pik3r3.S & 3.06 & $1.94 \mathrm{E}-07$ \\
\hline tdrp.L & 3.06 & $1.05 \mathrm{E}-13$ \\
\hline Xelaev18044942m.g & 3.06 & $3.49 \mathrm{E}-02$ \\
\hline slc43a2.S & 3.05 & $6.22 \mathrm{E}-09$ \\
\hline Xelaev18016487m.g & 3.04 & $1.56 \mathrm{E}-02$ \\
\hline tars2.L & 3.04 & $6.88 \mathrm{E}-04$ \\
\hline dlx6.S & 3.03 & $5.56 \mathrm{E}-05$ \\
\hline Xelaev18037602m.g & 3.02 & $1.61 \mathrm{E}-06$ \\
\hline nrp1.L & 3.02 & $2.34 \mathrm{E}-04$ \\
\hline prr5l.S & 3.01 & $4.80 \mathrm{E}-02$ \\
\hline creb3/1.S & 3.01 & $9.56 \mathrm{E}-06$ \\
\hline$d k k 1 . S$ & 3.00 & $6.83 \mathrm{E}-04$ \\
\hline dact1.L & 3.00 & $5.75 \mathrm{E}-05$ \\
\hline hes3.3.L & 2.99 & $1.44 \mathrm{E}-07$ \\
\hline dpys/3.S & 2.99 & $3.05 \mathrm{E}-10$ \\
\hline$n k x 6-1 . L$ & 2.98 & $3.08 \mathrm{E}-02$ \\
\hline Xelaev18027769m.g & 2.97 & $5.10 \mathrm{E}-04$ \\
\hline larp6-like.1.S & 2.97 & $3.56 \mathrm{E}-04$ \\
\hline fam222a.L & 2.97 & $2.38 \mathrm{E}-09$ \\
\hline ptch2.S & 2.96 & $2.16 \mathrm{E}-02$ \\
\hline sulf1.L & 2.96 & $1.63 \mathrm{E}-09$ \\
\hline $\operatorname{vim} . L$ & 2.96 & $5.23 \mathrm{E}-11$ \\
\hline plekhg1.L & 2.96 & $3.84 \mathrm{E}-05$ \\
\hline cdc42ep4.S & 2.95 & $2.20 \mathrm{E}-05$ \\
\hline ap/nr.L & 2.95 & $3.63 \mathrm{E}-11$ \\
\hline enpp1.L & 2.95 & $3.54 \mathrm{E}-02$ \\
\hline mex3a.S & 2.95 & $3.58 \mathrm{E}-04$ \\
\hline epn1.S & 2.94 & $1.66 \mathrm{E}-07$ \\
\hline$a p 3 b 2 . L$ & 2.94 & $2.21 \mathrm{E}-03$ \\
\hline
\end{tabular}




\begin{tabular}{|c|c|c|}
\hline LOC100498215-like.L & 2.94 & $2.65 \mathrm{E}-10$ \\
\hline reep5.S & 2.93 & $5.80 \mathrm{E}-19$ \\
\hline tmem169.S & 2.93 & $1.72 \mathrm{E}-03$ \\
\hline fgfr4.L & 2.91 & $3.07 E-11$ \\
\hline rev1.L & 2.88 & $1.01 \mathrm{E}-04$ \\
\hline tox2.S & 2.88 & $3.84 \mathrm{E}-06$ \\
\hline rundc3a.L & 2.87 & $2.03 E-03$ \\
\hline st3gal6.L & 2.87 & 8.07E-05 \\
\hline spsb4-like.L & 2.87 & $1.41 \mathrm{E}-03$ \\
\hline six3.L & 2.87 & $1.33 \mathrm{E}-12$ \\
\hline map2.L & 2.86 & $3.50 \mathrm{E}-03$ \\
\hline ag1.S & 2.86 & $6.90 \mathrm{E}-08$ \\
\hline rgs9bp.S & 2.86 & $2.73 \mathrm{E}-02$ \\
\hline cited2.L & 2.85 & $1.64 \mathrm{E}-05$ \\
\hline slco5a1.L & 2.85 & $2.67 \mathrm{E}-06$ \\
\hline tmem116.S & 2.82 & $7.76 \mathrm{E}-04$ \\
\hline cemip.S & 2.82 & $2.31 \mathrm{E}-02$ \\
\hline numbl.L & 2.80 & $3.53 \mathrm{E}-04$ \\
\hline Xelaev18003796m.g & 2.79 & $1.70 \mathrm{E}-02$ \\
\hline Xelaev18019210m.g & 2.79 & $2.68 \mathrm{E}-05$ \\
\hline gsta1.L & 2.78 & $3.66 \mathrm{E}-02$ \\
\hline akap12.L & 2.78 & $3.13 \mathrm{E}-06$ \\
\hline fam222a.S & 2.77 & $8.29 \mathrm{E}-04$ \\
\hline znf219.L & 2.76 & $7.32 \mathrm{E}-03$ \\
\hline sparc.L & 2.76 & $5.09 \mathrm{E}-03$ \\
\hline slc27a3.L & 2.76 & $4.21 \mathrm{E}-20$ \\
\hline psat1.S & 2.75 & 2.26E-02 \\
\hline$m e x 3 b . L$ & 2.75 & $6.51 \mathrm{E}-05$ \\
\hline sox4.S & 2.75 & $1.74 \mathrm{E}-03$ \\
\hline rnf165.S & 2.74 & $6.87 \mathrm{E}-10$ \\
\hline plxnb1.S & 2.74 & $1.54 \mathrm{E}-04$ \\
\hline hes9-1.S & 2.74 & 4.64E-09 \\
\hline cep85I.S & 2.73 & $2.78 \mathrm{E}-03$ \\
\hline LOC100145446.S & 2.73 & $7.33 \mathrm{E}-03$ \\
\hline nipa1.L & 2.72 & $2.37 \mathrm{E}-04$ \\
\hline rpa2.S & 2.72 & $1.23 \mathrm{E}-02$ \\
\hline dgki.S & 2.72 & $4.10 \mathrm{E}-02$ \\
\hline twist1.L & 2.72 & $7.98 \mathrm{E}-06$ \\
\hline
\end{tabular}




\begin{tabular}{|c|c|c|}
\hline hes5.1.L & 2.72 & 1.79E-03 \\
\hline sox21-like.S & 2.71 & 9.94E-03 \\
\hline otx2.L & 2.71 & 7.06E-04 \\
\hline LOC733561.S & 2.71 & $6.98 \mathrm{E}-08$ \\
\hline LOC100485511-like.S & 2.71 & $1.36 \mathrm{E}-02$ \\
\hline znf608.S & 2.70 & $1.28 \mathrm{E}-13$ \\
\hline vcan.L & 2.69 & $6.14 \mathrm{E}-04$ \\
\hline$\overline{i r f 1 . L}$ & 2.69 & $8.14 \mathrm{E}-03$ \\
\hline ptpdc1.L & 2.68 & $4.18 \mathrm{E}-04$ \\
\hline crmp1.S & 2.68 & $1.24 \mathrm{E}-02$ \\
\hline enpp2.L & 2.67 & 5.83E-04 \\
\hline Irp4.S & 2.67 & $1.77 \mathrm{E}-05$ \\
\hline smarcc2.S & 2.67 & 4.94E-03 \\
\hline rgma.L & 2.65 & $1.14 \mathrm{E}-05$ \\
\hline LOC100492639-like.L & 2.65 & $2.79 \mathrm{E}-09$ \\
\hline Irwd1.L & 2.64 & $3.33 \mathrm{E}-14$ \\
\hline edn1.L & 2.63 & $4.61 \mathrm{E}-02$ \\
\hline fgf16.L & 2.63 & $6.52 \mathrm{E}-03$ \\
\hline $\operatorname{Im} x 1 b .1 . L$ & 2.63 & $1.33 \mathrm{E}-02$ \\
\hline sulf1.S & 2.62 & $2.77 \mathrm{E}-02$ \\
\hline pik3r3.L & 2.62 & $8.73 E-04$ \\
\hline tox3.L & 2.61 & $1.09 \mathrm{E}-03$ \\
\hline nckap5I.L & 2.61 & $1.69 \mathrm{E}-06$ \\
\hline$n k d 1 . S$ & 2.60 & $1.09 \mathrm{E}-02$ \\
\hline spry2.S & 2.60 & $2.61 \mathrm{E}-03$ \\
\hline ak3.S & 2.59 & $1.76 \mathrm{E}-06$ \\
\hline Xelaev18032779m.g & 2.59 & $2.10 \mathrm{E}-02$ \\
\hline gse1.L & 2.59 & $1.66 \mathrm{E}-07$ \\
\hline efnb2.S & 2.58 & $3.96 \mathrm{E}-04$ \\
\hline kcnq3.S & 2.58 & $5.85 \mathrm{E}-04$ \\
\hline Irch2.L & 2.58 & $2.50 \mathrm{E}-04$ \\
\hline ncam1.S & 2.58 & 8.07E-06 \\
\hline fam43a.S & 2.57 & $2.05 \mathrm{E}-02$ \\
\hline ckap2.S & 2.57 & $2.08 \mathrm{E}-03$ \\
\hline nxpe3.L & 2.56 & $1.79 \mathrm{E}-02$ \\
\hline s1pr5.L & 2.56 & $2.62 \mathrm{E}-03$ \\
\hline zic2.L & 2.56 & $1.01 \mathrm{E}-02$ \\
\hline ppp1r14b.S & 2.55 & $2.15 \mathrm{E}-04$ \\
\hline
\end{tabular}




\begin{tabular}{|c|c|c|}
\hline Xelaev18010427m.g & 2.55 & $8.48 \mathrm{E}-04$ \\
\hline $\operatorname{tacc1.L}$ & 2.55 & 7.07E-03 \\
\hline Xelaev18021928m.g & 2.55 & $2.97 \mathrm{E}-05$ \\
\hline fbxo10.L & 2.54 & $9.01 \mathrm{E}-06$ \\
\hline cdk5r1.S & 2.54 & $3.59 \mathrm{E}-04$ \\
\hline hnrnpll.S & 2.54 & $3.81 \mathrm{E}-06$ \\
\hline hes8.S & 2.53 & 3.04E-08 \\
\hline sox9.S & 2.53 & $6.34 \mathrm{E}-03$ \\
\hline LOC100489393.L & 2.53 & $1.41 \mathrm{E}-04$ \\
\hline magi1.S & 2.53 & $3.94 \mathrm{E}-05$ \\
\hline fgfr1.S & 2.51 & $2.81 \mathrm{E}-08$ \\
\hline cdc42bpa.S & 2.51 & $3.10 \mathrm{E}-06$ \\
\hline hes9-1.L & 2.51 & $2.37 \mathrm{E}-02$ \\
\hline pou5f3.2.S & 2.51 & 4.97E-03 \\
\hline homer3.S & 2.51 & $3.75 \mathrm{E}-02$ \\
\hline $\operatorname{aim11.S}$ & 2.50 & $1.03 \mathrm{E}-11$ \\
\hline jag1.L & 2.50 & $2.69 \mathrm{E}-04$ \\
\hline fdft1.L & 2.49 & $6.78 \mathrm{E}-03$ \\
\hline$p k d c c . L$ & 2.48 & $2.05 \mathrm{E}-09$ \\
\hline ttc9.L & 2.48 & $4.45 \mathrm{E}-08$ \\
\hline cbfb.S & 2.48 & $7.74 \mathrm{E}-08$ \\
\hline ir $\times 2 . L$ & 2.47 & $1.70 \mathrm{E}-07$ \\
\hline fndc3b.S & 2.47 & $2.10 \mathrm{E}-02$ \\
\hline cyp27c1.S & 2.47 & 1.67E-04 \\
\hline cdc42ep4.L & 2.46 & $2.28 \mathrm{E}-02$ \\
\hline sh2b2.L & 2.46 & $1.19 \mathrm{E}-02$ \\
\hline riok3.S & 2.46 & $3.63 \mathrm{E}-11$ \\
\hline dnajc11.S & 2.45 & $2.92 \mathrm{E}-06$ \\
\hline Xelaev18015889m.g & 2.45 & $1.80 \mathrm{E}-02$ \\
\hline$s t \times 2 . L$ & 2.45 & $3.15 \mathrm{E}-05$ \\
\hline krt19.S & 2.44 & $2.85 \mathrm{E}-04$ \\
\hline aplnr.S & 2.44 & $2.78 \mathrm{E}-02$ \\
\hline scamp4.S & 2.44 & $3.30 \mathrm{E}-03$ \\
\hline tmem169.L & 2.44 & $1.41 \mathrm{E}-02$ \\
\hline pdgfb.L & 2.44 & $7.32 \mathrm{E}-03$ \\
\hline hn1.S & 2.43 & $8.06 \mathrm{E}-10$ \\
\hline Xetrov90002103m.L & 2.43 & $1.50 \mathrm{E}-02$ \\
\hline $\operatorname{lm} \times 1 b .1 . S$ & 2.42 & $3.86 \mathrm{E}-02$ \\
\hline
\end{tabular}




\begin{tabular}{|c|c|c|}
\hline sfrp2.L & 2.42 & $9.20 \mathrm{E}-07$ \\
\hline Xelaev18028757m.g & 2.42 & $1.18 \mathrm{E}-02$ \\
\hline tpbg.S & 2.42 & $2.75 \mathrm{E}-03$ \\
\hline sesn1.S & 2.41 & 3.77E-09 \\
\hline egln2.L & 2.41 & 1.49E-02 \\
\hline pmp22.S & 2.40 & $1.59 \mathrm{E}-04$ \\
\hline Xetrov90025030m.L & 2.40 & 2.27E-03 \\
\hline slit1.L & 2.40 & $3.43 \mathrm{E}-02$ \\
\hline plxnb1.L & 2.40 & 4.79E-06 \\
\hline zbtb16.S & 2.39 & $1.09 \mathrm{E}-02$ \\
\hline $\operatorname{lims1.L}$ & 2.39 & 8.02E-03 \\
\hline Xelaev18029838m.g & 2.38 & 8.02E-03 \\
\hline ngfr.L & 2.37 & $3.17 \mathrm{E}-04$ \\
\hline rbm38.L & 2.37 & $2.10 \mathrm{E}-08$ \\
\hline hdac4.L & 2.37 & $2.58 \mathrm{E}-02$ \\
\hline trmt6.S & 2.36 & 1.79E-05 \\
\hline spon1.L & 2.36 & $8.84 \mathrm{E}-03$ \\
\hline gas7.L & 2.35 & $3.15 \mathrm{E}-02$ \\
\hline fmn2.L & 2.34 & $4.16 \mathrm{E}-02$ \\
\hline dbndd1.L & 2.34 & $2.41 \mathrm{E}-02$ \\
\hline mprip.L & 2.33 & $6.68 \mathrm{E}-03$ \\
\hline nuak1.L & 2.33 & $8.38 \mathrm{E}-03$ \\
\hline pag1.S & 2.33 & $2.63 \mathrm{E}-03$ \\
\hline rcan1.S & 2.33 & $2.27 \mathrm{E}-03$ \\
\hline lysmd2.S & 2.32 & $2.68 \mathrm{E}-02$ \\
\hline hunk.S & 2.32 & $8.85 \mathrm{E}-03$ \\
\hline stk40.L & 2.31 & $2.15 \mathrm{E}-04$ \\
\hline draxin.S & 2.31 & $7.33 \mathrm{E}-03$ \\
\hline mycn.L & 2.30 & $8.31 \mathrm{E}-03$ \\
\hline prph.L & 2.30 & $5.18 \mathrm{E}-04$ \\
\hline hmox1.L & 2.30 & $3.86 \mathrm{E}-10$ \\
\hline notch1.L & 2.30 & $4.59 \mathrm{E}-05$ \\
\hline rcor2.L & 2.30 & $5.43 \mathrm{E}-08$ \\
\hline bach2.S & 2.30 & $8.17 \mathrm{E}-03$ \\
\hline bcar1.S & 2.29 & $1.01 \mathrm{E}-02$ \\
\hline smarca5.L & 2.29 & $1.03 \mathrm{E}-06$ \\
\hline ephb3.S & 2.29 & 3.37E-09 \\
\hline cybrd1.L & 2.29 & $1.34 \mathrm{E}-07$ \\
\hline
\end{tabular}




\begin{tabular}{|c|c|c|}
\hline rassf2.L & 2.29 & 7.91E-04 \\
\hline irx1.L & 2.28 & $1.01 \mathrm{E}-03$ \\
\hline zc4h2.L & 2.28 & $1.18 \mathrm{E}-05$ \\
\hline$z f h x 4 . L$ & 2.28 & $2.81 \mathrm{E}-05$ \\
\hline Xetrov90018501m.S & 2.28 & $2.68 \mathrm{E}-02$ \\
\hline psat1.L & 2.28 & $3.50 \mathrm{E}-03$ \\
\hline Ihfp/2.L & 2.27 & $1.01 \mathrm{E}-02$ \\
\hline fam69a.L & 2.27 & $6.59 \mathrm{E}-03$ \\
\hline dock6-like.L & 2.27 & $4.41 \mathrm{E}-06$ \\
\hline fam43a.L & 2.27 & $1.16 \mathrm{E}-02$ \\
\hline LOC100492804.L & 2.27 & $4.29 \mathrm{E}-03$ \\
\hline gatm.S & 2.26 & $1.60 \mathrm{E}-02$ \\
\hline Xelaev18024436m.g & 2.25 & $1.27 \mathrm{E}-03$ \\
\hline ubac1.S & 2.25 & $1.21 \mathrm{E}-02$ \\
\hline smim3.S & 2.25 & $1.28 \mathrm{E}-02$ \\
\hline Xelaev18030439m.g & 2.24 & $4.68 \mathrm{E}-02$ \\
\hline ptprt.L & 2.24 & 4.46E-02 \\
\hline Xelaev18015058m.g & 2.24 & $1.53 \mathrm{E}-12$ \\
\hline fat1.S & 2.23 & 3.77E-09 \\
\hline$f z d 2 . L$ & 2.23 & $3.70 \mathrm{E}-06$ \\
\hline tfap2a.S & 2.22 & 1.16E-05 \\
\hline spry2.L & 2.21 & $4.27 \mathrm{E}-02$ \\
\hline hyal2.S & 2.20 & $7.57 \mathrm{E}-03$ \\
\hline hes6.1.S & 2.20 & $2.43 \mathrm{E}-03$ \\
\hline rcor1.L & 2.19 & $4.65 \mathrm{E}-08$ \\
\hline hand2.S & 2.19 & $5.45 \mathrm{E}-04$ \\
\hline dicer1.S & 2.18 & 2.09E-06 \\
\hline Xetrov90009914m.S & 2.18 & $5.28 \mathrm{E}-03$ \\
\hline rai2.S & 2.18 & $2.28 \mathrm{E}-02$ \\
\hline calb2.S & 2.17 & $1.57 \mathrm{E}-02$ \\
\hline LOC100144919.L & 2.17 & $2.33 \mathrm{E}-05$ \\
\hline$t . S$ & 2.17 & 4.49E-02 \\
\hline rfk.S & 2.16 & 1.49E-02 \\
\hline ndst1.S & 2.16 & $1.31 \mathrm{E}-04$ \\
\hline rasgef1b.L & 2.16 & $1.57 \mathrm{E}-06$ \\
\hline nkain1.L & 2.16 & $3.02 \mathrm{E}-02$ \\
\hline cdc25b.L & 2.15 & $4.39 \mathrm{E}-04$ \\
\hline hes3.3.S & 2.15 & 1.06E-03 \\
\hline
\end{tabular}




\begin{tabular}{|c|c|c|}
\hline ano8.L & 2.14 & $3.71 \mathrm{E}-02$ \\
\hline Ifng.L & 2.14 & 6.97E-06 \\
\hline afap1/1.L & 2.14 & $3.54 \mathrm{E}-11$ \\
\hline cdc25b.S & 2.14 & $3.12 \mathrm{E}-04$ \\
\hline nrarp.L & 2.14 & $4.87 \mathrm{E}-04$ \\
\hline cdc42se2-like.1.S & 2.13 & $3.42 \mathrm{E}-03$ \\
\hline cdc42se2-like.1.L & 2.12 & 6.47E-04 \\
\hline cmip.L & 2.12 & $4.41 \mathrm{E}-03$ \\
\hline fgd1.S & 2.12 & $3.11 \mathrm{E}-03$ \\
\hline nceh1.L & 2.11 & $2.82 \mathrm{E}-02$ \\
\hline SOX4.L & 2.11 & $2.23 \mathrm{E}-02$ \\
\hline acer2.L & 2.11 & $6.78 \mathrm{E}-04$ \\
\hline LOC733709.S & 2.11 & $4.71 \mathrm{E}-02$ \\
\hline sh3glb2.S & 2.11 & $4.86 \mathrm{E}-02$ \\
\hline ets2.S & 2.10 & $4.15 \mathrm{E}-02$ \\
\hline srgap2.S & 2.10 & $2.81 \mathrm{E}-05$ \\
\hline dusp4.S & 2.10 & $7.46 \mathrm{E}-03$ \\
\hline st3gal2.1.L & 2.10 & $8.50 \mathrm{E}-04$ \\
\hline lifr. $L$ & 2.09 & $1.46 \mathrm{E}-03$ \\
\hline ccnt2.S & 2.09 & $6.53 \mathrm{E}-09$ \\
\hline $\operatorname{atg} 9 b . L$ & 2.09 & $3.05 \mathrm{E}-02$ \\
\hline fgfr4.S & 2.09 & $1.32 \mathrm{E}-10$ \\
\hline wasf3.L & 2.09 & $1.22 \mathrm{E}-02$ \\
\hline snx7.S & 2.07 & $7.40 \mathrm{E}-03$ \\
\hline sox2.S & 2.07 & $4.30 \mathrm{E}-02$ \\
\hline LOC100145450.S & 2.07 & $4.11 \mathrm{E}-03$ \\
\hline tfap2a.L & 2.06 & $2.57 \mathrm{E}-08$ \\
\hline Xelaev18041479m.g & 2.06 & $2.14 \mathrm{E}-02$ \\
\hline slc23a2.S & 2.06 & $2.58 \mathrm{E}-02$ \\
\hline prickle1.S & 2.05 & $1.83 \mathrm{E}-04$ \\
\hline plekha3.L & 2.05 & $1.99 \mathrm{E}-02$ \\
\hline myt1.S & 2.05 & $3.35 \mathrm{E}-04$ \\
\hline cuedc1.L & 2.05 & $3.61 \mathrm{E}-03$ \\
\hline rap2b.S & 2.05 & $5.86 \mathrm{E}-14$ \\
\hline clic5.S & 2.04 & $7.53 \mathrm{E}-03$ \\
\hline Ipar4.S & 2.04 & 8.07E-05 \\
\hline gpatch11.L & 2.04 & $3.76 \mathrm{E}-05$ \\
\hline memo1.L & 2.03 & 2.34E-03 \\
\hline
\end{tabular}




\begin{tabular}{|l|c|c|}
\hline Xetrov90025030m.S & 2.03 & $6.19 \mathrm{E}-03$ \\
\hline aen.L & 2.03 & $1.82 \mathrm{E}-03$ \\
\hline eya2.S & 2.02 & $4.82 \mathrm{E}-02$ \\
\hline myt1.L & 2.02 & $7.32 \mathrm{E}-03$ \\
\hline auts2.L & 2.02 & $2.25 \mathrm{E}-04$ \\
\hline il5ra.S & 2.02 & $4.44 \mathrm{E}-02$ \\
\hline gas1.S & 2.01 & $2.52 \mathrm{E}-03$ \\
\hline arhgap4.L & 2.01 & $2.86 \mathrm{E}-03$ \\
\hline kiaa1755-like.S & 2.01 & $1.37 \mathrm{E}-02$ \\
\hline dynll2.L & 2.00 & $3.27 \mathrm{E}-03$ \\
\hline mex3b.S & 2.00 & $3.12 \mathrm{E}-03$ \\
\hline mapkapk5.L & 2.00 & $6.71 \mathrm{E}-06$ \\
\hline amotl2.L & 2.00 & $2.50 \mathrm{E}-04$ \\
\hline
\end{tabular}

\subsubsection{Candidate gene list for the RNA sequencing analysis of Brg1}

\section{knock-down affected genes}

Given are the genes which are differentially expressed between Ptf1a-GR + cMO overexpressing animal caps over Ptf1a-GR + Brg1MO overexpressing caps in two individual replicates. Given are the gene ID, the $\log _{2} \mathrm{FC}$ activation over $\mathrm{CC}$ and the $\mathrm{p}$ value.

Table S20: Summary of genes Ptf1a + cMO vs. Ptf1a + Brg1MO (downregulated by Brg1 knock-down)

\begin{tabular}{|l|c|c|}
\hline ID & log2FC & P-value \\
\hline cacnb1.S & 5.02 & $2.69 \mathrm{E}-03$ \\
\hline paqr9.L & 4.94 & $4.11 \mathrm{E}-05$ \\
\hline hoxc3.L & 4.94 & $7.45 \mathrm{E}-03$ \\
\hline nhlh1.S & 4.72 & $2.24 \mathrm{E}-10$ \\
\hline nhlh1.L & 4.43 & $9.66 \mathrm{E}-16$ \\
\hline scrt1.L & 4.28 & $1.51 \mathrm{E}-02$ \\
\hline tubb2b.S & 4.20 & $2.13 \mathrm{E}-03$ \\
\hline Imo2.S & 4.19 & $3.31 \mathrm{E}-06$ \\
\hline Xetrov90016831m.L & 4.06 & $1.94 \mathrm{E}-02$ \\
\hline dnajc18.L & 3.94 & $2.43 \mathrm{E}-02$ \\
\hline Xelaev18024654m.g & 3.94 & $3.29 \mathrm{E}-05$ \\
\hline Xelaev18024653m.g & 3.87 & $1.22 \mathrm{E}-02$ \\
\hline tvp23a.S & 3.84 & $1.93 \mathrm{E}-02$ \\
\hline Xelaev18044320m.g & 3.78 & $1.77 \mathrm{E}-02$ \\
\hline
\end{tabular}




\begin{tabular}{|c|c|c|}
\hline Xelaev18047333m.g & 3.74 & $1.22 \mathrm{E}-02$ \\
\hline ppp1r9b.L & 3.35 & $2.35 \mathrm{E}-03$ \\
\hline six3.S & 3.25 & 1.60E-03 \\
\hline emilin1.L & 3.17 & $4.11 \mathrm{E}-05$ \\
\hline Ibx1.S & 3.11 & $3.27 \mathrm{E}-03$ \\
\hline LOC100496628-like.S & 2.92 & 7.87E-04 \\
\hline arhgap4.L & 2.86 & 9.77E-06 \\
\hline pax6.S & 2.86 & $2.27 \mathrm{E}-03$ \\
\hline flnc.S & 2.80 & $1.26 \mathrm{E}-02$ \\
\hline manbal.S & 2.75 & $1.61 \mathrm{E}-02$ \\
\hline tox2.S & 2.67 & $1.09 \mathrm{E}-06$ \\
\hline gria1-like.1.S & 2.61 & $6.01 \mathrm{E}-03$ \\
\hline Xelaev18011187m.g & 2.53 & 7.05E-03 \\
\hline mef2d.S & 2.53 & $6.95 \mathrm{E}-03$ \\
\hline gch1.L & 2.51 & 3.91E-02 \\
\hline trabd2a.L & 2.46 & $2.27 \mathrm{E}-03$ \\
\hline kiaa1755.L & 2.44 & $8.21 \mathrm{E}-03$ \\
\hline Xelaev18004886m.g & 2.41 & $1.74 \mathrm{E}-02$ \\
\hline cep85I.L & 2.39 & $3.87 \mathrm{E}-02$ \\
\hline tmem35.L & 2.32 & $2.50 \mathrm{E}-02$ \\
\hline traf4.S & 2.27 & $2.56 \mathrm{E}-02$ \\
\hline slc43a2.L & 2.25 & $2.33 \mathrm{E}-02$ \\
\hline slc43a2.S & 2.23 & $6.30 \mathrm{E}-05$ \\
\hline ptch1.L & 2.21 & $1.81 \mathrm{E}-02$ \\
\hline ypel1.S & 2.15 & $2.90 \mathrm{E}-03$ \\
\hline prph.L & 2.14 & $1.37 \mathrm{E}-02$ \\
\hline guca1a.S & 2.11 & $2.87 \mathrm{E}-02$ \\
\hline nes.L & 2.11 & $4.42 \mathrm{E}-03$ \\
\hline rufy3.L & 1.98 & $7.73 \mathrm{E}-05$ \\
\hline plxna3.L & 1.97 & $1.18 \mathrm{E}-02$ \\
\hline nr5a2.S & 1.96 & $4.78 \mathrm{E}-02$ \\
\hline nes.S & 1.95 & $8.62 \mathrm{E}-03$ \\
\hline dpy19/4.L & 1.93 & 1.97E-02 \\
\hline pgs1.S & 1.93 & $2.13 \mathrm{E}-03$ \\
\hline$z e b 2 . L$ & 1.85 & $2.76 \mathrm{E}-03$ \\
\hline st18.L & 1.84 & $4.09 \mathrm{E}-02$ \\
\hline nckap5I.L & 1.83 & $6.95 \mathrm{E}-03$ \\
\hline nfe2/3.L & 1.80 & $8.97 \mathrm{E}-03$ \\
\hline
\end{tabular}




\begin{tabular}{|c|c|c|}
\hline acy3.L & 1.79 & 4.42E-03 \\
\hline sfrp2.S & 1.78 & $3.34 \mathrm{E}-10$ \\
\hline sfrp2.L & 1.77 & $6.49 \mathrm{E}-03$ \\
\hline fbrs/1.S & 1.73 & $1.26 \mathrm{E}-03$ \\
\hline slc25a28.L & 1.71 & $2.47 E-04$ \\
\hline$z e b 2 . S$ & 1.68 & $5.64 \mathrm{E}-03$ \\
\hline prph.S & 1.66 & $3.23 \mathrm{E}-06$ \\
\hline tubb3.S & 1.66 & $1.90 \mathrm{E}-02$ \\
\hline slc7a7.L & 1.62 & $4.11 \mathrm{E}-03$ \\
\hline pak3.S & 1.60 & $1.58 \mathrm{E}-02$ \\
\hline Irrc45.L & 1.54 & $1.26 \mathrm{E}-02$ \\
\hline plk3.L & 1.54 & $2.35 \mathrm{E}-03$ \\
\hline C1orf194.L & 1.54 & $2.92 \mathrm{E}-02$ \\
\hline ak2.S & 1.51 & 1.97E-02 \\
\hline klhdc7a.S & 1.47 & 2.92E-02 \\
\hline bcr.L & 1.45 & $3.51 \mathrm{E}-02$ \\
\hline six3.L & 1.44 & $1.05 \mathrm{E}-02$ \\
\hline slc7a8.S & 1.42 & $4.44 \mathrm{E}-02$ \\
\hline sp8.S & 1.41 & $2.50 \mathrm{E}-02$ \\
\hline ifitm10-like.L & 1.40 & $3.63 \mathrm{E}-02$ \\
\hline $\mathrm{nrm} . \mathrm{L}$ & 1.34 & 4.87E-04 \\
\hline spata18-like.S & 1.34 & $2.50 \mathrm{E}-02$ \\
\hline skida1.S & 1.31 & $2.70 \mathrm{E}-02$ \\
\hline tuba1b.L & 1.30 & $3.73 \mathrm{E}-02$ \\
\hline fbrs/1.L & 1.22 & $1.34 \mathrm{E}-02$ \\
\hline sp7.L & 1.20 & 3.37E-02 \\
\hline ypel1.L & 1.19 & $1.51 \mathrm{E}-02$ \\
\hline asna1.S & 1.19 & $2.11 \mathrm{E}-02$ \\
\hline c19orf43.S & 1.14 & $1.17 \mathrm{E}-02$ \\
\hline pxmp4.L & 1.10 & $6.33 \mathrm{E}-03$ \\
\hline slc7a6.L & 1.08 & $4.92 \mathrm{E}-02$ \\
\hline ube2r2.L & 1.08 & $2.29 \mathrm{E}-02$ \\
\hline ubap2.S & 1.05 & $3.20 \mathrm{E}-02$ \\
\hline gmnn.L & 1.04 & $3.08 \mathrm{E}-02$ \\
\hline$m f s d 9 . L$ & 1.02 & 4.43E-02 \\
\hline
\end{tabular}


Table S21: Summary of genes Ptf1a + cMO vs. Ptf1a + Brg1MO (upregulated by Brg1 knock-down)

\begin{tabular}{|c|c|c|}
\hline ID & $\log 2 \mathrm{FC}$ & P-value \\
\hline LOC101730771-like.L & -4.84 & $8.60 \mathrm{E}-03$ \\
\hline larp6-like.1.S & -3.74 & $7.21 \mathrm{E}-05$ \\
\hline LOC100485511-like.S & -3.55 & 1.06E-02 \\
\hline eda2r.L & -3.12 & 8.29E-07 \\
\hline pou5f3.2.L & -3.07 & $6.55 \mathrm{E}-06$ \\
\hline itga2b.1-like.S & -3.00 & 7.74E-03 \\
\hline tars2.L & -2.97 & 1.04E-02 \\
\hline LOC733561.S & -2.67 & $2.54 \mathrm{E}-06$ \\
\hline LOC100495756-like.S & -2.61 & $2.54 \mathrm{E}-06$ \\
\hline pou5f3.2.S & -2.57 & 3.49E-02 \\
\hline nipa1.L & -2.37 & $1.58 \mathrm{E}-02$ \\
\hline rev1.L & -2.28 & $2.96 \mathrm{E}-02$ \\
\hline hand2.S & -2.19 & $6.95 \mathrm{E}-03$ \\
\hline riok3.S & -2.14 & $3.32 \mathrm{E}-07$ \\
\hline Irwd1.L & -2.12 & $6.11 \mathrm{E}-08$ \\
\hline nudt22.L & -1.82 & $1.31 \mathrm{E}-03$ \\
\hline ctnnal1.S & -1.79 & $1.18 \mathrm{E}-02$ \\
\hline dicer1.S & -1.79 & $2.27 \mathrm{E}-03$ \\
\hline LOC100144919.L & -1.76 & $9.06 \mathrm{E}-03$ \\
\hline tinf2.L & -1.71 & $3.27 \mathrm{E}-03$ \\
\hline Xelaev18043774m.g & -1.70 & $2.40 \mathrm{E}-02$ \\
\hline Xelaev18034615m.g & -1.68 & $2.40 \mathrm{E}-03$ \\
\hline Xelaev18043947m.g & -1.66 & 3.75E-02 \\
\hline tex2.2.L & -1.63 & $7.05 E-03$ \\
\hline itpr1.S & -1.58 & 4.47E-05 \\
\hline skil.S & -1.56 & $2.41 \mathrm{E}-02$ \\
\hline $\operatorname{cog} 5 . L$ & -1.51 & $1.51 \mathrm{E}-02$ \\
\hline cdo1.S & -1.50 & $1.51 \mathrm{E}-02$ \\
\hline Xelaev18034617m.g & -1.49 & $1.31 \mathrm{E}-02$ \\
\hline srsf5.S & -1.45 & 3.35E-02 \\
\hline kitlg.S & -1.44 & $6.33 \mathrm{E}-03$ \\
\hline ska3.L & -1.43 & $7.49 \mathrm{E}-03$ \\
\hline rest.S & -1.43 & $2.76 \mathrm{E}-03$ \\
\hline pou5f3.1.S & -1.39 & $1.93 \mathrm{E}-02$ \\
\hline pawr.S & -1.37 & 9.92E-03 \\
\hline
\end{tabular}




\begin{tabular}{|l|c|c|}
\hline plekhn1.S & -1.35 & $2.57 \mathrm{E}-03$ \\
\hline ccdc30.L & -1.35 & $2.35 \mathrm{E}-03$ \\
\hline pdgfa.S & -1.32 & $3.44 \mathrm{E}-02$ \\
\hline rnf168.L & -1.26 & $1.56 \mathrm{E}-02$ \\
\hline gata2.L & -1.25 & $1.41 \mathrm{E}-04$ \\
\hline cdkn2aip.L & -1.22 & $3.51 \mathrm{E}-02$ \\
\hline Xelaev18036901m.g & -1.20 & $1.04 \mathrm{E}-03$ \\
\hline marveld3.L & -1.19 & $2.92 \mathrm{E}-02$ \\
\hline ccnt2.S & -1.17 & $3.19 \mathrm{E}-02$ \\
\hline tob1.S & -1.17 & $3.91 \mathrm{E}-03$ \\
\hline snrpb2.S & -1.17 & $2.13 \mathrm{E}-03$ \\
\hline bambi.S & -1.15 & $7.73 \mathrm{E}-05$ \\
\hline ccna2.S & -1.15 & $3.05 \mathrm{E}-03$ \\
\hline znf654.L & -1.14 & $2.02 \mathrm{E}-02$ \\
\hline hal.1.L & -1.13 & $6.72 \mathrm{E}-03$ \\
\hline Xelaev18024066m.g & -1.13 & $4.66 \mathrm{E}-02$ \\
\hline Xelaev18023103m.g & -1.12 & $4.09 \mathrm{E}-03$ \\
\hline tp53.L & -1.12 & $2.67 \mathrm{E}-02$ \\
\hline tacc2.L & -1.10 & $1.99 \mathrm{E}-02$ \\
\hline ptpn2.S & -1.02 & $1.58 \mathrm{E}-02$ \\
\hline bambi.L & & $3.97 \mathrm{E}-02$ \\
\hline
\end{tabular}

\subsection{Quantification of the BiFC assay}

Given are the corresponding N- and C-terminal Venus-constructs used for the BiFC analysis, the relative amount of EYFP positive cells compared to the total number of mRFP positive cells, the total number of mRFP positive cells, and the SEM value

TableS22: Quantification of the BiFC

\begin{tabular}{|l|l|c|c|r|}
\hline Vc-construct & Vn-construct & $\begin{array}{c}\text { EYFP pos cells } \\
\text { (in \%) }\end{array}$ & $\begin{array}{c}\text { total } \\
\text { number } \\
\text { of cells }\end{array}$ & \multicolumn{1}{|c|}{ SEM } \\
\hline \multirow{3}{*}{ Ptf1a-Vn } & E12-Vc & $80 \%$ & 165 & $20 \%$ \\
\cline { 2 - 5 } & Rbpj-Vc & $84 \%$ & 1118 & $9 \%$ \\
\cline { 2 - 5 } & Prdm13-Vc & $3 \%$ & 657 & $2 \%$ \\
\hline
\end{tabular}




\begin{tabular}{|l|l|c|c|r|}
\hline \multirow{4}{*}{ Ptf1aW224A/W242A-Vn } & E12-Vc & $71 \%$ & 196 & $20 \%$ \\
\cline { 2 - 5 } & Rbpj-Vc & $0 \%$ & 659 & $0 \%$ \\
\cline { 2 - 5 } & Prdm13-Vc & $3 \%$ & 494 & $3 \%$ \\
\hline \multirow{3}{*}{ Ptf1aT243A-Vn } & E12-Vc & $73 \%$ & 59 & $20 \%$ \\
\cline { 2 - 5 } & Rbpj-Vc & $84 \%$ & 1182 & $16 \%$ \\
\cline { 2 - 5 } & Prdm13-Vc & $49 \%$ & 697 & $2 \%$ \\
\hline \multirow{3}{*}{ Ptf1aT243E-Vn } & E12-Vc & $100 \%$ & 57 & $0 \%$ \\
\cline { 2 - 5 } & Rbpj-Vc & $88 \%$ & 336 & $2 \%$ \\
\cline { 2 - 5 } & Prdm13-Vc & $40 \%$ & 777 & $24 \%$ \\
\hline
\end{tabular}




\section{Curriculum vitae}

Name:

Date of birth:

Place of birth:

Address:

Nationality:

E-Mail:
Sven Richts

29.01.1988

Werl, Germany

Stumpfe Eiche 1, 37077, Göttingen

German

sven.richts@med.uni-goettingen.de

Since 2014

PhD studies: "Mechanisms underlying the temporal and selective induction of Ptf1a target genes"

Georg-August Universität Göttingen, Göttingen, Germany

$\mathrm{PhD}$. candidate in GGNB program Genes and Development

$\underline{2010-2013}$

Master studies: "Characterization of Ptf1a protein domains during neurogenesis"

Georg-August Universität Göttingen, Göttingen, Germany

Master of Science in Developmental, Neural and Behavioral Biology (DNB)

Area of specialization: Developmental and Cellular Biology

$\underline{2007-2010}$

Bachelor studies: "Untersuchungen zur Funktion von Dachshund im

Mehlkäfer Tribolium Castaneum"

Georg-August Universität Göttingen, Göttingen, Germany

Bachelor of Science in Biology

$\underline{1998-2007}$

Abitur: Städtisches Marien-Gymnasium Werl, Werl, Germany 